\title{
Role of intestinal dysbiosis on gut colonization by bacterial pathogens
}

Thesis submitted for the degree of Doctor of Biotechnology

Author: Ana Djukovic

Director: Dr. Carles Úbeda Morant July, 2017 



\title{
Role of intestinal dysbiosis on
}

\section{gut colonization by bacterial}

\section{pathogens}

\author{
Author: \\ Ana Djukovic \\ Director: \\ Dr. Carles Úbeda Morant \\ Tutor: \\ Dr. Rafael Sirera Pérez
}

A thesis submitted for the degree of

Doctor of Biotechnology

$$
\text { July, } 2017
$$



Mojoj mami 



\section{Acknowledgements}

First of all, I would like to thank Carles for putting his trust in me and giving me the opportunity to do my $\mathrm{PhD}$ in his laboratory. During these five years I cried, I laughed, I doubted myself, but I certainly learned a lot. Thank you for teaching me to think critically, for teaching me to endure, for moving my limits, for pointing me to the importance of decision about the kind of scientist I want to be, and above all, for helping me grow as a person.

I am deeply grateful to all the people at the rabbit farm of the Polytechnic University of Valencia. Especially to Enrique, Eugenio, and Luis- your dedication to science served me as inspiration. I will never forget the farm in the middle of the fields of Alboraya!

I would also like to thank to Jose and his group for opening the doors of their lab at Segorbe and helping me with my first experiments on mice.

Many thanks to Microbiology and Hematology departments at Hospital La Fe, Valencia. Thank you Eva and Salva for all the help and advices.

I am deeply grateful to Joao for receiving me at his lab and introducing me to the world of bioinformatics and modeling. I am thrilled that I will be part of your lab and I am sure we will be doing great things!

A very special thanks to all my labbies, my "becarios". To Ana E, Pedro, Raul, Leo, Maria, Bea, Marc, Anny, Jorge, Dani, Artacho, who unselfishly shared their knowledge and support when I needed it most. I will never forget all the Friday lunches at Chinos, dancing at Matisse, all the confessions we shared and comfort you gave me; and to the new members of this large family- Alba, Majda, Galo, Irving, Carlos, Bob and Majotime spent with you was "trambolic"! To Ana D and Mariam- thank you for coffee breaks, for late talks, for strange humor that spiced every conversation and for love and support you gave me. And the last, but not the least, to my girls Sandrine, Bea and Alejandra. I loved being a part of our small group, where every one of us is different, but yet we complement each other perfectly. Thank you for all the help in lab, but more importantly, for being kind, warm, and protective. It was a privilege to share this time with you. You made it unforgettable. 
To my friends from Serbia- to Sanja, Tamara, Mina, Cebal who made me miss my origins even more; and also to Josep, Ana, Joan, Pilar and Ana Cristina for helping me fall in love with Spain, and giving me another home.

Hvala, hvala, beskonačno hvala mojim Rajičićima, mlađima i starijima, mojim Kuprešanima, mojim Tanaskovićima, mojim Collinsima, mojoj porodici, mojoj bazi. Sanja, hvala ti za duge razgovore, za beskrajno razumevanje, i za ljubav i podršku koji mi daju snage. Mama hvala ti. Ti si znala pre mene šta i koliko mogu. Gurala si, podržavala, hrabrila i čuvala, a najviše si propatila zbog moje ambicije. Hvala ti za čaj od hibiskusa posle sankanja, za šetnje po Ušcu prvog januara, za svu vodu bačenu niz stepenice, za sve kafe u ispitnom roku, za iskrenost i kad boli, a najviše, za svu nežnost ovog sveta. Sve je lako kad imam vas!

To Ivan, for saying "yes" when everybody said "no". For filling that empty space and becoming my friend, my brother and my family.

Mil gracias a mi familia española. Gracias Ramón, Pepa, Pepe y Ruth por aceptarme y hacerme sentir como en casa. Por fin tengo mi pueblo!

Y al final, a mí Ramoncito. Nada de esto sería posible sin ti. Gracias por intentar entender cada uno de experimentos, por esperarme cada noche en frente de $\mathrm{La} \mathrm{Fe}$, por proporcionarme refugio cuando lo necesitaba, por ser sincero y critico, por todos los abrazos, por ser noble, por ser solidario, por las risas que despejaban las preocupaciones, por desear nada más que mi felicidad, por todos los sacrificios que hemos hecho juntos para llegar aquí. Porque entre dos podemos con todo. Entre los dos lo hemos conseguido.

This is for all of us scientists: curious, persistent, hardworking, missing-beer-because-ofan-experiment-people. Do not miss too many beers! 




\section{Abstract}

The intestinal tract of virtually any metazoan, including mammals, is colonized with a complex microbial community to which we refer as intestinal or gut microbiota. One of the roles of the healthy intestinal microbiota is to protect the host against gut colonization with pathogenic bacteria through a phenomenon known as colonization resistance (CR). Dysbiosis of the intestinal microbiota, usually as a result of an antibiotic treatment, may lead to the disruption of the $\mathrm{CR}$, and subsequent colonization with bacterial pathogens. Interestingly, CR is affected differently by antibiotics of different spectrum, suggesting that some members of the microbiota participate in CR while others are dispensable. Moreover, once established in the intestine, bacterial pathogens may reach high levels and disseminate into the bloodstream of the host provoking infection. However, and despite the importance, the role of the microbiota dysbiosis on the gut colonization by many bacterial pathogens, such as multidrug resistant Enterobacteriaceae, has not been elucidated: the members of the microbiota that confer $\mathrm{CR}$ and factors that promote colonization remain mostly unknown.

The general aim of this thesis has been to improve the understanding of the role of the microbiota dysbiosis in gut colonization by bacterial pathogens. For this purpose, 3 projects have been established. In the first project we tried to elucidate the role of the microbiota dysbiosis on colonization by multidrug resistant Enterobacteriaceae (MRE) in mice. MRE, such as Escherichia coli and Klebsiella pneumoniae, are among the leading causes of morbidity and mortality worldwide. Infections with MRE usually begin by the colonization of the intestinal tract. However, little is known about clinical factors associated with MRE colonization or which members of microbiota could prevent colonization and subsequent infection with MRE. In order to elucidate the role of the microbiota dysbiosis on mice colonization with MRE, we treated mice with several antibiotics and infected them with a multidrug resistant strain of $K$. pneumoniae (MRKP). Our results indicate that persistent microbiota dysbiosis, as a result of antibiotic treatment, promotes MRKP intestinal colonization in mice. Importantly, we were able to demonstrate that the restoration of the levels of 2 bacterial genera (Lactobacillus and Barnesiella), depleted by antibiotic therapy, significantly diminish the ability of MRKP to colonize the intestinal tract. 
In the second project we investigated the risk factors and members of the microbiota associated with the MRE colonization in hospitalized patients. MRE infections represent a great threat for hospitalized patients. Specifically, acute leukemia patients are often colonized with MRE, probably due to the impaired CR as a result of intensive antibiotic treatments these patients receive. Notably, we did not identify any antibiotic that enhance intestinal colonization by MRE. In contrast, we identified systematic administration of beta-lactams, when the patient is colonized with MRE strain sensitive to beta-lactams, as one of the factors associated with the reduction in MRE colonization levels. On the other hand, administration of beta-lactams when the patient is colonized with MRE beta-lactam-resistant strain does not enhance MRE intestinal colonization. Among commensal bacteria associated with changes in MRE colonization levels, we identified higher abundance of the genus Coprobacillus, 1 operational taxonomical unit (OTU) of the genus Lactobacillus and 2 Bacteroides OTUs to be associated with a decrease of MRE fecal levels in acute leukemia patients, suggesting that these commensal bacteria may have a protective role against MRE.

In the third project we studied the role of the microbiota dysbiosis on the development of Epizootic Rabbit Enteropathy (ERE). ERE is a severe gastrointestinal disease with a high percentage of mortality that occurs in young rabbits during first weeks post-weaning. ERE rabbits have been shown to suffer microbiota dysbiosis during the development of the disease. Moreover, the disease could be reproduced by contact between healthy and sick animals and by administration of cecal contents from ERE rabbits to healthy rabbits, suggesting that a pathogenic agent may be involved in the development of this intestinal pathology, although no causative agent has been identified until now. Here, we demonstrated that the major changes in the microbiota composition observed after the initiation of ERE cannot be detected in rabbits that will develop ERE before the disease onset. Moreover, the only change that could be detected before the development of ERE was the higher presence of a novel species within the genus Clostridium in rabbits that will develop ERE on the very day of the disease onset, suggesting that this bacterium could have a major role in the initiation of ERE. 


\section{Resumen}

El tracto intestinal de prácticamente cualquier metazoo, incluidos los mamíferos, está colonizado por una compleja comunidad microbiana a la que nos referimos como microbiota intestinal. Uno de los papeles de la microbiota intestinal es proteger al huésped contra la colonización intestinal con bacterias patógenas a través de un fenómeno conocido como resistencia a la colonización (RC). La disbiosis de la microbiota intestinal, a menudo como resultado de un tratamiento antibiótico, puede conducir a la alteración de la RC y posterior colonización por patógenos bacterianos. Curiosamente, $\mathrm{RC}$ es afectada de manera distinta por antibióticos de espectro diferente, lo que sugiere que algunos miembros de la microbiota participan en $\mathrm{RC}$, mientras que otros son dispensables. Además, una vez establecidos en el intestino, los patógenos bacterianos pueden alcanzar niveles altos y diseminarse en el torrente sanguíneo del huésped provocando infección. Sin embargo, y pese a su importancia, el papel de la disbiosis de la microbiota en la colonización intestinal por muchos patógenos bacterianos, como son las Enterobacterias multirresistentes, no se ha esclarecido: los miembros de la microbiota que confieren $\mathrm{RC}$ y los factores que promueven la colonización siguen siendo desconocidos.

El objetivo general de esta tesis ha sido mejorar la comprensión del papel de disbiosis de la microbiota en la colonización intestinal por patógenos bacterianos. Para ello se han establecido tres proyectos. En el primer proyecto investigamos el papel de disbiosis de la microbiota intestinal en la colonización por Enterobacterias multiresistentes (MRE) en ratones. MRE, como Escherichia coli y Klebsiella pneumoniae, están entre las principales causas de morbilidad y mortalidad en todo el mundo. Las infecciones con MRE generalmente comienzan por la colonización del tracto intestinal. Sin embargo, se sabe poco sobre los factores clínicos asociados con la colonización de MRE o que miembros de la microbiota podrían prevenir la colonización y la consecuente infección con MRE. Con el fin de esclarecer el papel de la disbiosis de la microbiota intestinal en la colonización con MRE en ratones, se trataron ratones con varios antibióticos y se infectaron con una cepa multiresistente de $K$. pneumoniae (MRKP). Nuestros resultados indican que la persistente disbiosis microbiana, resultante del tratamiento antibiótico, promueve la colonización intestinal con MRKP en ratones. Es importante destacar que hemos podido demostrar que la restauración de niveles de 2 
géneros bacterianos (Lactobacillus y Barnesiella), afectados por el tratamiento con antibióticos, disminuye significativamente la capacidad de MRKP para colonizar el tracto intestinal de ratones.

En el segundo proyecto investigamos los factores de riesgo y los miembros de la microbiota asociados con la colonización por MRE en pacientes hospitalizados. Las infecciones por MRE representan una gran amenaza para los pacientes hospitalizados. Específicamente, MRE a menudo colonizan los pacientes con leucemia aguda, probablemente debido a que la $\mathrm{RC}$ está alterada como resultado de tratamientos antibióticos intensivos recibidos por estos pacientes. Cabe destacar que no identificamos ningún antibiótico que promueva la colonización intestinal por MRE. Al contrario, hemos identificado la administración sistemática de beta-lactámicos, cuando el paciente se coloniza con la cepa MRE sensible a beta-lactámicos, como uno de los factores asociados con la reducción de los niveles de colonización intestinal por MRE. Por otro lado, la administración de beta- lactámicos cuando el paciente está colonizado con una cepa resistente a beta-lactámicos no aumenta los niveles intestinales de MRE. Entre las bacterias comensales asociadas con cambios en los niveles de colonización por MRE, identificamos el género Coprobacillus, una unidad taxonómica operativa (OTU) del género Lactobacillus y dos OTUs del género Bacteroides estar asociadas con una disminución de los niveles fecales de MRE en pacientes con leucemia aguda, lo que sugiere que estas bacterias comensales pueden tener un papel protector contra el MRE.

En el tercer proyecto investigamos el papel de la disbiosis microbiana en desarollo de Enteropatía Epizoótica de Conejo (ERE). ERE es una enfermedad gastrointestinal severa con un alto porcentaje de mortalidad que ocurre en conejos jóvenes durante las primeras semanas después del destete. Se ha demostrado que los conejos con ERE sufren disbiosis microbiana después del inicio de la enfermedad, aunque no está claro el papel de la disbiosis en el desarollo de la enfermedad. Además, la enfermedad puede ser reproducida por contacto entre animales sanos y enfermos y por la administración del contenido cecal de conejos con ERE a conejos sanos, lo que sugiere que un agente patógeno podría estar implicado en el desarrollo de esta patología intestinal, aunque hasta ahora no se ha logrado identificar ningún agente causal. Aquí, hemos demostrado que los principales cambios en la composición de la microbiota observada después de la iniciación de ERE no se puede detectar en los conejos antes de la aparición de la enfermedad. Por otra parte, el único cambio que se pudo detectar antes de desarollo de la enfermedad fue la mayor presencia de una especie nueva dentro del 
género Clostridium en conejos que desarrollaran ERE el mismo día de la aparición de la enfermedad, lo que sugiere que esta bacteria podría tener un papel importante en el inicio de ERE. 


\section{Resum}

El tracte intestinal de pràcticament qualsevol metazoo, inclosos els mamífers, està colonitzat per una complexa comunitat microbiana a la qual ens referim com microbiota intestinal. Un dels papers de la microbiota intestinal és protegir a l'hoste contra la colonització intestinal amb bacteris patògens a través d'un fenomen conegut com a resistència a la colonització ( $\mathrm{RC})$. La disbiosis de la microbiota intestinal, frecuentment com a resultat d'un tractament antibiòtic, pot conduir a l'alteració de la RC i posterior colonització per patògens bacterians. Curiosament, RC és afectada de manera diferent pels antibiòtics d'espectre diferent, la qual cosa suggereix que alguns membres de la microbiota participen en $\mathrm{RC}$, mentre que uns altres són dispensables. A més, una vegada establerts en l'intestí, els patògens bacterians poden aconseguir nivells alts $\mathrm{i}$ disseminar-se en el torrent sanguini de l'hoste provocant la infecció. No obstant això, i malgrat la seva importància, el paper de la disbiosis de la microbiota en la colonització intestinal per molts patògens bacterians, com són les Enterobacteries multirresistentes, no s'ha esclarit: els membres de la microbiota que confereixen $\mathrm{RC}$ i els factors que promouen la colonització segueixen sent desconeguts.

L'objectiu general d'aquesta tesi ha estat millorar la comprensió del paper de la disbiosis de la microbiota en la colonització intestinal per patògens bacterians. Per a això s'han establert tres projectes. En el primer projecte vam investigar el paper de la disbiosis de la microbiota intestinal en la colonització per Enterobacteries multiresistentes (MRE) en ratolins. MRE, com Escherichia coli i Klebsiella pneumoniae, estan entre les principals causes de morbiditat i mortalitat a tot el món. Les infeccions amb MRE generalment comencen per la colonització del tracte intestinal. No obstant això, se sap poc sobre els factors clínics associats amb la colonització de MRE o que membres de la microbiota podrien previndre la colonització i la subsegüent infecció amb MRE. Amb la finalitat d'esclarir el paper de la disbiosis de la microbiota intestinal en la colonització amb MRE en ratolins, vam tractar ratolins amb diversos antibiòtics i els vam infectar amb un cep multiresistente de $K$. pneumoniae (MRKP). Els nostres resultats indiquen que la persistent disbiosis microbiana, com a resultat del tractament antibiòtic, promou la colonització intestinal amb MRKP en ratolins. És important destacar que hem pogut demostrar que la restauració de nivells de 2 gèneres bacterians (Lactobacillus i 
Barnesiella), afectats pel tractament amb antibiòtics, disminueix significativament la capacitat de MRKP per colonitzar el tracte intestinal de ratolins.

En el segon projecte, investiguem els factors de risc i els membres de la microbiota associats amb la colonització per MRE en pacients hospitalitzats. Les infeccions per MRE representen una gran amenaça per als pacients hospitalitzats. Específicament, MRE sovint colonitza els pacients amb leucèmia aguda, probablement a causa de que la $\mathrm{RC}$ està alterada com a resultat de tractaments antibiòtics intensius rebuts per aquests pacients. Cal destacar que no vam identificar cap antibiòtic que promogui la colonització intestinal per MRE. Al contrari, hem identificat l'administració sistemàtica de beta-lactamics, quan el pacient es colonitza amb un cep MRE sensible a betalactamics, com un dels factors associats amb la reducció dels nivells de colonització intestinal per MRE. D'altra banda, l'administració de beta-lactamics quan el pacient està colonitzat amb un cep resistent a beta-lactámics no augmenta els nivells intestinals de MRE. Entre les bacteries comensals associades amb canvis en els nivells de colonització per MRE, hem identificat el gènere Coprobacillus, una unitat taxonómica operativa (OTU) del gènere Lactobacillus i dos OTUs del gènere Bacteroides estar associades amb una disminució dels nivells fecals de MRE en pacients amb leucèmia aguda, la qual cosa suggereix que aquests bacteries comensals poden tenir un paper protector contra el MRE.

En el tercer projecte, vam investigar el paper de la disbiosis microbiana en desenvolupament de l'Enteropatía Epizoótica de Conill (ERE). ERE és una malaltia gastrointestinal severa amb un alt percentatge de mortalitat que ocorre en conills joves durant les primeres setmanes després del deslleti. S'ha demostrat que els conills amb ERE sofreixen disbiosis microbiana després de l'inici de la malaltia, encara que no és clar el paper de la disbiosis en el desenvolupament de la malaltia. A més, la malaltia pot ser reproduïda per contacte entre animals sans i malalts i per l'administració del contingut cecal de conills amb ERE a conills sans, la qual cosa suggereix que un agent patogen podria estar implicat en el desenvolupament d'aquesta patologia intestinal, encara que fins ara no s'ha aconseguit identificar cap agent causal. Aquí, hem demostrat que els principals canvis en la composició de la microbiota observada després de la iniciació de ERE no es poden detectar en els conills abans de l'aparició de la malaltia. D'altra banda, l'únic canvi que es va poder detectar abans del desenvolupament de la malaltia va ser la major presència d'una espècie nova dins del gènere Clostridium en conills que van desenvolupar ERE, el mateix dia de l'aparició de la malaltia, la qual cosa suggereix que aquest bacteri podria tenir un paper important en l'inici de ERE. 



\section{Table of Contents}

1 Introduction

1.1 The intestinal microbiota. 1

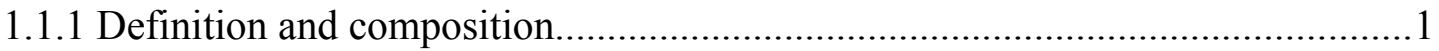

1.1.1.1 Temporal variability of the intestinal microbiota....................................2

1.1.1.2 Spatial variability of the intestinal microbiota........................................ 3

1.1.1.3 Microbiota variability among different host species.................................5

1.1.1.4 External factors that affect composition of the intestinal microbiota...........6

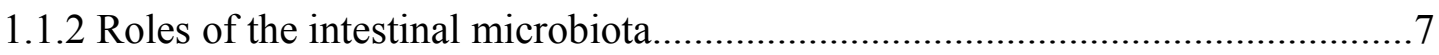

1.1.2.1 Effect of the intestinal microbiota on the host metabolism........................8

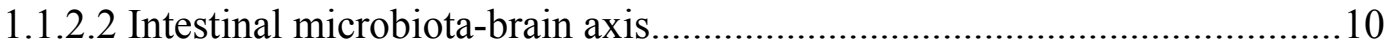

1.1.2.3 Effect of the intestinal microbiota on maturation of the immune system...11

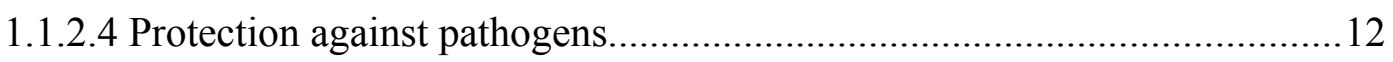

1.1.2.4.1 Protection mediated through microbiota induction of the immune

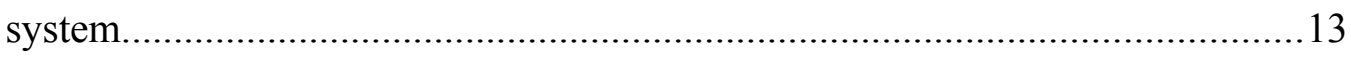

1.1.2.4.2 Direct protection against pathogens conferred by the intestinal microbiota

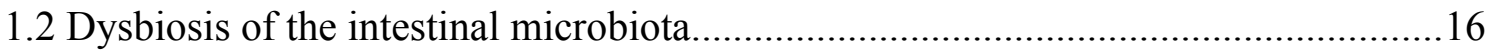

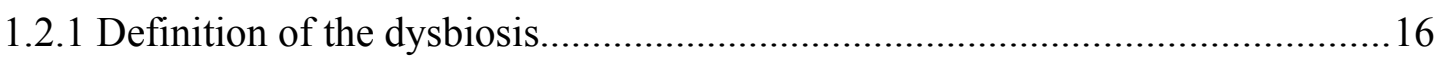

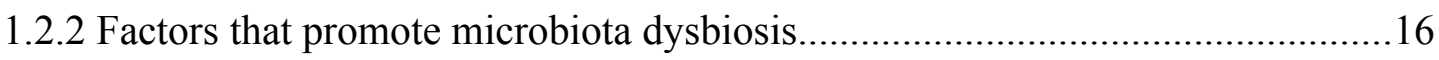

1.2.2.1 Diet as a promoter of microbiota dysbiosis.............................................16

1.2.2.2 Inflammation as a promoter of microbiota dysbiosis..............................17

1.2.2.3 Antibiotic treatment as a promoter of microbiota dysbiosis......................17

1.2.3 The effect of the dysbiosis on the host's health.................................................19

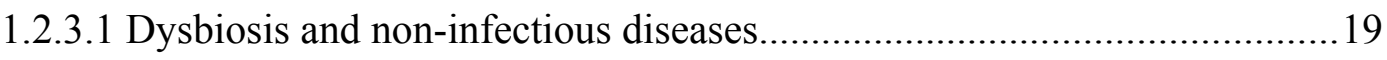

1.2.3.2 Dysbiosis and colonization of the intestinal tract by pathogens................20

1.2.3.2.1 Gut colonization by multidrug resistant Enterobacteriaceae (MRE)..21

1.2.3.2.2 Dysbiosis as a consequence of colonization with intestinal

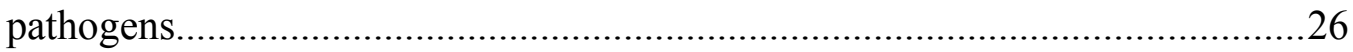

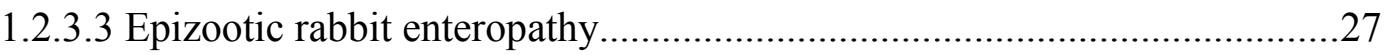




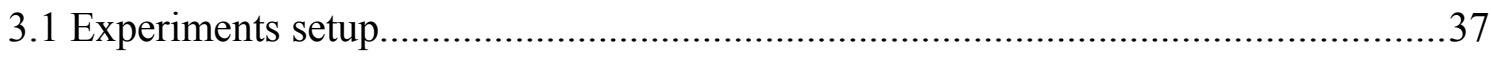

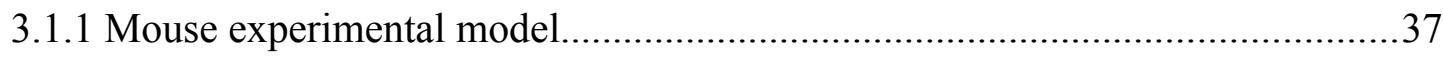

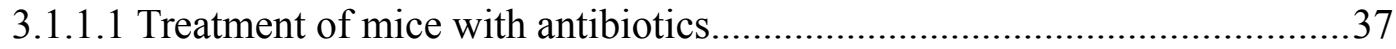

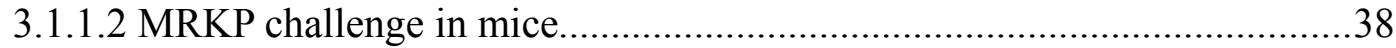

3.1.1.3 Fecal transplant and administration of specific commensal bacteria to mice.

3.1.1.3.1 Preparation of commensal bacterial mix obtained by cultivation of fecal samples from untreated mice in different cultivation media. 40

3.1.1.3.2 Preparation of commensal bacterial mix obtained by isolation of different bacterial strains isolated from fecal samples of untreated mice...........42

3.1.2 Fecal sample collection and MRE levels analysis in hospitalized patients........42

3.1.3 Experiments in rabbits for elucidating the role of dysbiosis in ERE.................43

3.1.3.1 Food deprivation experiment...................................................................44

3.1.3.2 Infection of healthy rabbits with the putative infectious agent isolated from ERE rabbits

3.2 Isolation and characterization of intestinal bacteria from mice and rabbits...............46

3.2.1 Cultivation and isolation of the intestinal bacteria............................................46

3.2.2 16S rRNA gene PCR of isolated intestinal bacteria........................................47

3.2.3 Obtaining the 16S rRNA gene sequence of the gut isolated bacteria using the

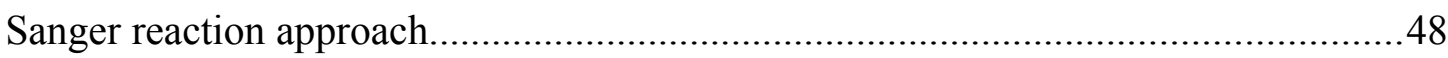

3.2.4 Taxonomic identification of isolated bacteria using the 16S rRNA sequence obtained with the Sanger reaction

3.3 Detection, isolation and characterization of antibiotic resistant pathogens .49

3.3.1 Detection of the levels of colonization with MRKP in mice. .49

3.3.2 Detection of MRE fecal levels in patients 
3.3.3 Characterization of MRE strains from patients' fecal samples........................50

3.3.3.1 Taxonomic confirmation of MRE strains by Maldi-TOF MS....................50

3.3.3.2 Determination of antibiotic resistance patterns of MRE strains using the Vitek 2 System

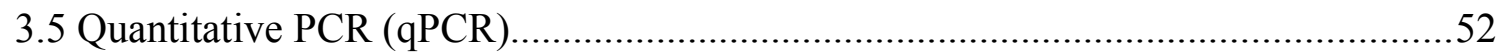

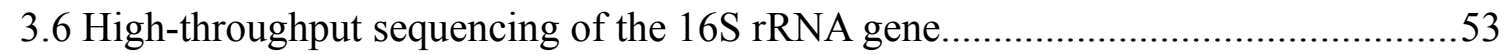

3.6.1 16S rDNA high-throughput sequencing using the 454 platform........................53

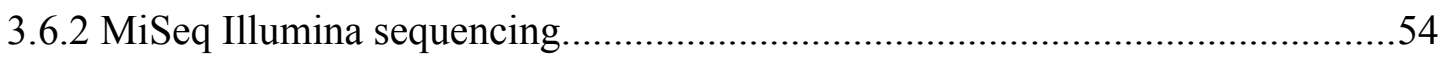

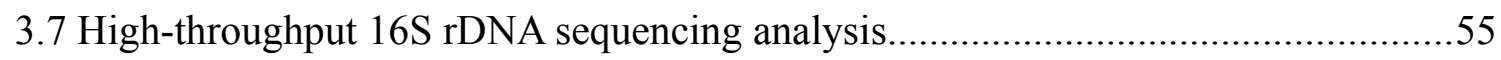

3.7.1 Analysis of sequences obtained using the 454 platform...................................55

3.7.2 Analysis of 16S rRNA sequences obtained using the MiSeq Illumina

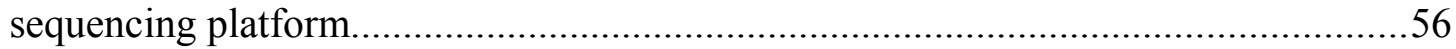

3.7.3 Principal Coordinate of Analysis and Hierarchical clustering.........................57

3.8 Nextera sequencing of the genome of the Clostridium strain identified as a potential promoter of ERE in rabbits

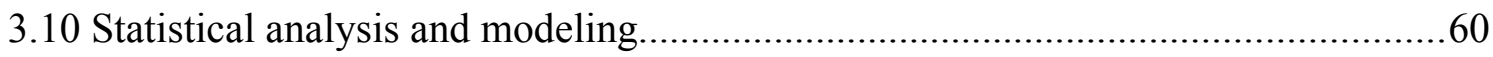

3.10.1 Statistical analysis applied in the mice studies.

3.10.2 Statistical analysis applied for identification of clinical factors associated with MRE colonization levels in patients with leukemia.

3.10.3 Application of the Lotka-Volterra (LV) model for the identification of commensal bacteria associated with resistance to MRE colonization in hospitalized patients

3.10.4 Statistical analysis applied in the study with rabbits. 66

4 Results. 
4.1 Microbiota dysbiosis and MRE colonization and persistence in mice.

4.1.1 The effect of antibiotic treatment on MRE intestinal colonization and persistence

4.1.2 Long-lasting changes in the microbiota composition as a result of antibiotic treatment promote MRKP intestinal colonization.

4.1.3 Restoration of the $\mathrm{CR}$ and suppression of MRKP colonization. 84

4.1.3.1 Restoration of the microbiota with a fecal transplant suppresses MRKP intestinal colonization.

4.1.3.2 Effect of the administration of fecal microbiota anaerobic cultures in restoring CR against MRKP after antibiotic treatment......................................85

4.1.3.3 Identification of bacteria that could provide CR against MRKP. .87

4.1.3.4 A commensal bacterial mix partially restores MRKP colonization resistance after antibiotic therapy.....

4.1.3.5 Partial microbiota recovery after administration of the bacterial mix.

4.2 Microbiota dysbiosis and MRE colonization in hospitalized patients 95

4.2.1 Prevalence and characterization of MRE in acute leukemia patients...............95

4.2.2 Non-microbiota variables associated with MRE intestinal levels.....................98

4.2.2.1 Impact of non-temporal variables on MRE intestinal levels.....................99

4.2.2.2 Impact of temporal variables on MRE intestinal levels. 102

4.2.2.3 Clinical parameters associated with the intestinal colonization levels in the first MRE positive sample.

4.2.3 Identification of bacteria associated with protection against MRE colonization in hospitalized patients.

4.3 The role of the microbiota dysbiosis in ERE 123

4.3.1 ERE is characterized by dysbiosis of the intestinal microbiota.

4.3.2 Microbiota dynamics after the weaning and before the ERE onset in healthy and ERE rabbits 128

4.3.3 Comparison of the microbiota composition of ERE rabbits on the day of disease onset with age-matched healthy littermate controls 132 
4.3.4 Higher sequencing coverage using Illumina platform identifies a unique microbiota change the day of ERE onset.

4.3.5 The effect of decreasing food consumption on ERE development and microbiota dysbiosis.

4.3.6 Analysis of the genome of the OTU31 classified as Clostridium and associated with ERE onset.

4.3.7 Attempt to reproduce ERE by administration of the C. cuniculi to healthy SPF rabbits.

5 Discussion.

5.1 Microbiota dysbiosis and MRE colonization and persistence in mice.

5.2 Microbiota dysbiosis and MRE colonization in hospitalized patients

5.3 The role of the microbiota dysbiosis in ERE.

6 Conclusions.

7 Abbreviations. 



\section{Introduction}

\subsection{The intestinal microbiota}

\subsubsection{Definition and composition}

The intestinal tract of virtually any metazoan, including mammals, is colonized with a complex microbial community to which we refer as intestinal or gut microbiota $(1,2)$. This ecosystem is comprised of members of all 3 domains of life: bacteria, archea, eukarya (fungi, yeasts and protozoa) and their viruses (3). However, due to the objectives and tasks performed during this thesis, we will focus on the bacterial components of this ecosystem. Bacteria represent the most numerous and the most studied microorganisms that colonize the intestinal tract $(4,5)$. They can reach concentrations of $10^{11}$ bacterial cells per gram of fecal content in the colon $(4,6)$. The majority of intestinal bacteria are strict anaerobes, which dominate facultative anaerobes and aerobes. Although it is suggested that there are around 50 bacterial phyla described to date, the gut microbiota of mammals is dominated by only 2 of them: Bacteroidetes and Firmicutes $(1,7,8)$. Other bacterial phyla, such as Proteobacteria, Verrucomicrobia, Actinobacteria and Fusobacteria are present in minor proportions. Although number of estimated bacterial species that inhabit intestinal tract (further called intestinal microbiota) vary among studies, it is generally accepted that it contains between 500 and 1,000 species $(8,9)$.

It is important to note that the composition of the intestinal microbiota is not homogeneous and varies highly during the lifetime, along the intestinal tract of the animal and even among different hosts $(1,6)$. Besides this natural variability, a wide range of external factors such as diet, geography, hygiene and antibiotic treatment can cause shifts in the microbiota composition. Some of these changes, termed dysbiosis, are associated with harmful effects for the host's health and may have long-term consequences leading to disease. In order to talk about dysbiotic state, we have to understand first variability that occurs naturally. 


\subsubsection{Temporal variability of the intestinal microbiota}

Colonization of the intestinal tract was thought to begin immediately after birth, although recent studies in which authors analyzed human placental microbiome (bacterial species and genes they encode) challenge this assumption $(10,11)$ In a study from 2016 Collado et al. (11) confirmed the presence of bacteria in placenta and in meconium (the newborn's first intestinal discharge). The authors suggested that there could be intrauterine colonization of the infant's gut with bacteria from mother's intestinal tract. These results concord with those obtained in another study published by Gosalbes et al. (12).

Following birth, intestinal microbiota is characterized by low diversity and changes in composition until it reaches a stable, adult-like state (13). During the first weeks infant's gut is colonized with facultative anaerobes including Staphylococcus, Streptococcus, and members of the family Enterobacteriaceae (14). These microbes consume the available oxygen and create an environment in which obligate anaerobes can grow. Various studies have shown that the initial composition of the microbiota could be affected by the mode of delivery $(15,16)$. While the intestinal microbiota of infants delivered vaginally shows similarities with mother's vaginal microbiota, infants delivered through cesarean section have a microbial composition more similar to the skin microbiota of their mothers (17). These results suggest that the microbiota of the environment (vaginal or skin) to which offspring is being exposed during birth could represent the seed for the future complex community that will be inhabiting their intestinal tract. It has been suggested that the mode of delivery, besides having an effect on microbiota composition, could be associated with celiac disease, obesity and asthma (17).

As mentioned before, first colonizers consume oxygen and produce new metabolites allowing succession of the primary microbial community with another one, comprised of strictly anaerobic bacteria such as bifidobacteria, Clostridium and Bacteroides $(13,15)$. This change in composition could be due to the introduction of breast or formula-feeding and represents first diet-related change in composition of the infant's intestinal microbiota. 
The second diet-related change in microbiota composition of a mammal occurs after weaning or transition to a solid food. Study performed on 605 infants from 5 European countries (Sweden, Scotland, Germany, Italy and Spain) showed that weaning was accompanied with a significant decrease in proportions of bifidobacteria, lactobacteria and enterobacteria, whereas some species within Firmicutes phylum increased (18).

Results from a study performed by Bergstrom et al. (13) suggest that the changes in the intestinal microbiota composition continue up to 36 months of age. During this period, the microbiota is converging towards adult-like state characterized by high levels of Firmicutes and Bacteroidetes and smaller fractions of Actinobacteria, Proteobacteria, and Verrucomicrobia.

A final set of age-related shifts in the composition and function of the gut microbiota occurs during old age. Age-related differences reported in a study performed by Odamaki et al. (19) include marked decrease in bifidobacteria in subjects older than 70 years.

\subsubsection{Spatial variability of the intestinal microbiota}

Besides variability in microbiota composition during the lifetime of an animal, there is also variability between different compartments of the gastrointestinal tract (GIT). Host physiology and intestinal microbiota are intimately connected. This is evident from the fact that each distinct anatomical region along the GIT is characterized by its own physiochemical conditions, and that these changing conditions exert a selective pressure on the microbiota $(20,21)$.

There is very limited insight in the composition of the human microbiota that resides in the small intestine due to the difficulty of sampling. In a study performed in 2011, Frank et al. (22) suggested that the wall of the distal small intestine is colonized by microbial populations that are not radically different from those of the large intestine, although some differences could be observed. Both environments are dominated by bacteria of the phyla Bacteroidetes and Firmicutes but differ in the relative proportions. Members of the class Bacilli (predominantly the Lactobacillales clade) were significantly more abundant in the small intestine than in the colon. In contrast, a variety of members 
of the Bacteroidetes and the family Lachnospiraceae were less abundant in the small intestine.

In another study performed on mucosal tissue collected along the colon and fecal samples collected from 3 healthy individuals Eckburg et al. (8) detected differences between the microbiota composition of feces and the one from the colonic mucosa. Eckburg et al. postulated that the fecal microbiota represents a combination of shed mucosal bacteria and a separate non-adherent luminal population. Indeed, Swidinski et al. (21) demonstrated that the microbiota present in the intestinal lumen differs significantly from the microbiota attached and embedded in the mucus layer as well as the microbiota present in the immediate proximity to the epithelium (Figure I1).
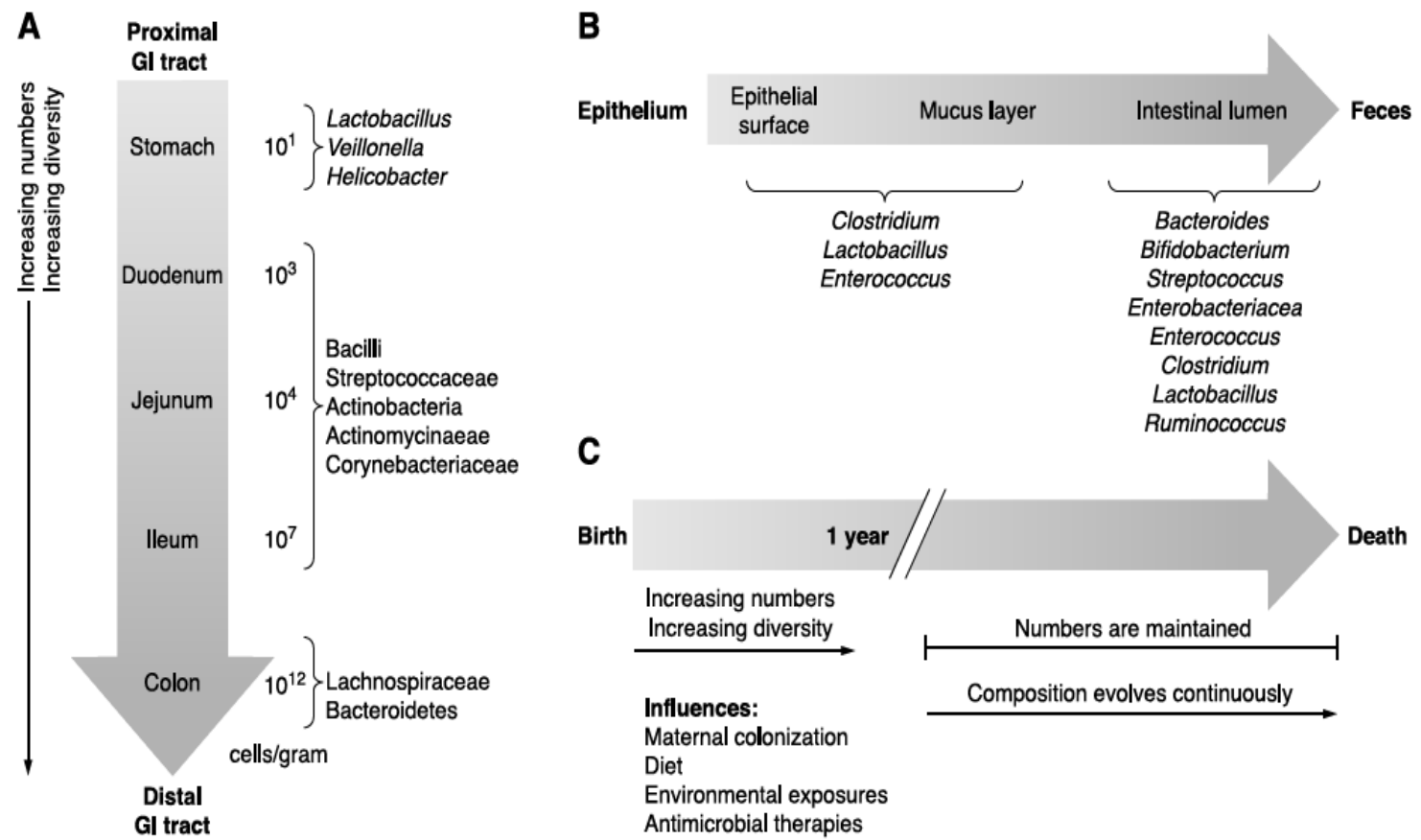

Figure I1. Spatial and temporal variability of intestinal microbiota composition. (A) Variations in microbial numbers and composition across the length of the GIT. (B) Transversal variations in microbial composition in the intestine. (C) Temporal aspects of microbiota establishment and maintenance. Adapted from Sekirov et al., Physiological Reviews, 2010.

For convenience, the majority of the studies whose objective is the assessment of the microbiota composition have been performed on fecal samples. Related with the thesis proposal, the fecal microbiota composition has been useful to identify specific changes in the microbiota that promote colonization by pathogens including 
vancomycin-resistant Enterococcus (VRE) (23) or Clostridium difficile (24), indicating that fecal samples can provide relevant information about changes in the microbiota that contribute to intestinal pathogen colonization.

\subsubsection{Microbiota variability among different host species}

Co-evolution is believed to occur in animal species whose parental care enables vertical transmission of whole gut communities, and where the properties of the microbial community confer a fitness advantage to the host (25). This co-evolution has been conferred from observations of bacterial host specificity.

Rawls et al. (26) performed a study in 2006 in which they showed differences in the microbiota composition between zebrafish and mice. Their results indicate that the intestinal microbiota of zebrafish and mice share 6 bacterial phyla, 5 of which are shared with the human microbiota. However, members of zebrafish microbiota within these shared phyla are distinct from those in mice and humans at more deeper phylogenetic resolution. Interestingly, the authors of this study isolated gut microbiota from zebrafish and mouse hosts and performed reciprocal transplantations of these consortia into germfree mice and zebrafish, respectively. Rawls et al. (26) demonstrated that vertebrate host can be colonized by the microbiota of another vertebrate species, but the ratios and proportions of the shared bacterial divisions will reflect the balance of the phyla natural to the host in which the microbiota is residing. They concluded that the gut environment of a particular host species "selects" which portions of an introduced microbial population will dominate and persist.

In a study performed in 2008, Ley et al. (25) analyzed the composition of the intestinal microbiota of 106 individual mammals representing 60 species from 13 taxonomic orders, including 17 non-human primates. The majority of detected bacteria in all mammals belonged to 2 phyla: Bacteroidetes and Firmicutes. However, each mammalian host harbored species not observed in any other. Overall, the fecal microbial communities of the same species hosts were more similar to each other than to those of different host species. The impact of host species on microbiota composition was evident, taking into account that some members of the same species analyzed, that 
contain similar microbiota, were living separately, therefore removing possible confounding effect of co-housing.

Studies performed in different animals, such as mice, rabbits or human, point to the variability in the microbiota composition as a result of co-evolution between host and its microbiota. However, and despite the differences, mice are commonly used as a model in order to clarify unknown aspects of the human microbiota. For example, related to the thesis, Buffie et al. (27) identified a protective role of the commensal bacterium Clostridium scindens against infections produced by $C$. difficile both in mice and humans, indicating that some results obtained from mice studies can be extrapolated to humans.

\subsubsection{External factors that affect composition of the intestinal microbiota}

As explained in previous sections, microbiota composition varies among different host species, along the GIT and during the lifetime. In addition, some external factors such as use of antibiotics or diet can also produce changes in the microbiota composition (28).

Although diet is known to be a driving force for the maturation of the intestinal microbiota (i.e. introduction of breast or formula feeding and solid food in infancy) and for the species specialization (animal species with similar diet have more similar microbiota compositions), it can also produce changes in the composition of the healthy, adult microbiota. In a study published in 2011, Wu et al. (29) demonstrated a high correlation between enterotypes (variants of the microbiota identified in humans) and long-term diet differences. The Bacteroides enterotype was associated with meat consumption, a feature characteristic of the Western diet, while the Prevotella enterotype was associated with a carbohydrate-based diet typical of agrarian societies. In another study, David et al. (30) demonstrated that the short-term consumption of diet composed entirely of animal or plant products alters the intestinal microbial community structure. The animal-based diet increases the abundance of bile-tolerant microorganisms (Alistipes, Bilophila, and Bacteroides) and decreases the levels of Firmicutes that metabolize dietary plant polysaccharides (Roseburia, Eubacterium rectale, and Ruminococcus bromii). Besides, it has been shown that tea phenolics and their 
derivatives repressed the growth of certain pathogenic bacteria such as $C$. perfringens, $C$. difficile, and Bacteroides spp., while they less severely affect commensal anaerobes (31). Dietary iron mostly from red meat and fortified cereals, or iron from cigarette smoking, can also change the gut bacteria composition (32). Elevated iron availability may increase the proliferation and virulence of gut bacteria and increase the permeability of the gut barrier. Fasting could also produce changes in microbiota composition. A recent study compared the responses of the gut microbiota to fasting across several vertebrate hosts (33). Interestingly, besides species-specific changes in microbiota composition as a result of food deprivation, authors observed other changes that were widespread among tested species. Specifically, tetrapods (toads, geckos, quail, mice) exhibited decrease in the abundances of Coprobacillus and Ruminococcus in the colon as a result of fasting.

Antibiotics represent another external factor that affects microbiota composition. Antibiotics are widely used as antimicrobial agents to treat infections caused by pathogenic bacteria. However, they do not only affect pathogens, but also commensal intestinal microbial communities. Effects of antibiotic treatments on the composition of the intestinal microbiota and the consequences that they have on health will be discussed in detail later in the section 1.2.2.3.

In order to understand how shifts in composition of the intestinal microbiota could lead to disease, it is important to understand the roles of the healthy intestinal microbiota, which will be explained in the following section.

\subsubsection{Roles of the intestinal microbiota}

Millions of years of mammalian-microbial co-evolution have led not only to tolerance of intestinal microbiota by the host but to interdependency among them. As a result, the intestinal microbiota plays a role in metabolic, nutritional, physiological and immunological processes in the host's body (20). In addition, the intestinal microbiota participates in the defense against pathogens, a phenomenon known as "colonization resistance". In the recent years, there has been an increase in the number of publications addressing the roles of the intestinal microbiota. These studies have allowed us to realize about the link that exists between the intestinal microbiota and health. Some of the roles 
of the intestinal microbiota in establishment and preservation of the healthy state of the host will be explained in the following sections.

\subsubsection{Effect of the intestinal microbiota on the host metabolism}

Various studies have shown that the intestinal microbiota maximizes caloric availability of nutrients ingested by the host (6). Mechanisms by which this occurs fall into 2 groups: extraction of additional calories from otherwise indigestible polysaccharides and promotion of nutrient uptake and utilization by modulation of the absorptive capacity of the intestinal epithelium.

Between $10 \%$ and $20 \%$ of ingested dietary carbohydrates are resistant to digestion in the small intestine. These non-digestible dietary carbohydrates enter the colon where one part is fermented by colonic bacteria to lactate, gases and short-chain fatty acids (SCFAs), including acetate, propionate and butyrate $(34,35)$. In mammals, butyrate serves as a predominant energy substrate for colonocytes and enterocytes. Propionate is primarily absorbed by the liver, where hepatocytes use it for gluconeogenesis, while acetate is released into peripheral tissues (Figure I2). In the human gut, members of Bacteroidetes phylum secrete high levels of acetate and propionate whereas those of the Firmicutes phylum generate large amounts of butyrate.

In addition to being able to break down indigestible polysaccharides to absorbable monosaccharides or SCFAs, the intestinal microbiota also modulates the uptake and deposition of dietary lipids. Bile acids (or bile salts) are steroid acids that are produced in the liver from cholesterol and secreted in bile where they facilitate the metabolism of dietary fat and the absorption of fat soluble vitamins and cholesterol (Figure I2) (36). Bile acids are secreted in form of bile salts conjugated with glycine and taurine. Members of genera Bacteroides, Eubacterium, and Clostridium have the ability to deconjugate bile salts and perform partial dehydroxylation producing secondary bile acids. This transformation is necessary not only for re-absorption of bile acids but also for performing their metabolic functions. Indeed, the gut microbiota, through effects on bile acid metabolism in the gut lumen, influence signaling pathways involved in energy and lipid metabolism. 
Besides facilitating access to nutrients, intestinal microbiota produce vitamins (B9, B12, vitamin K) and metabolizes xenobiotics (compounds of non-host origin that enter the gut with the diet), such as isoflavones, oxalates and choline $(28,36,37)$.

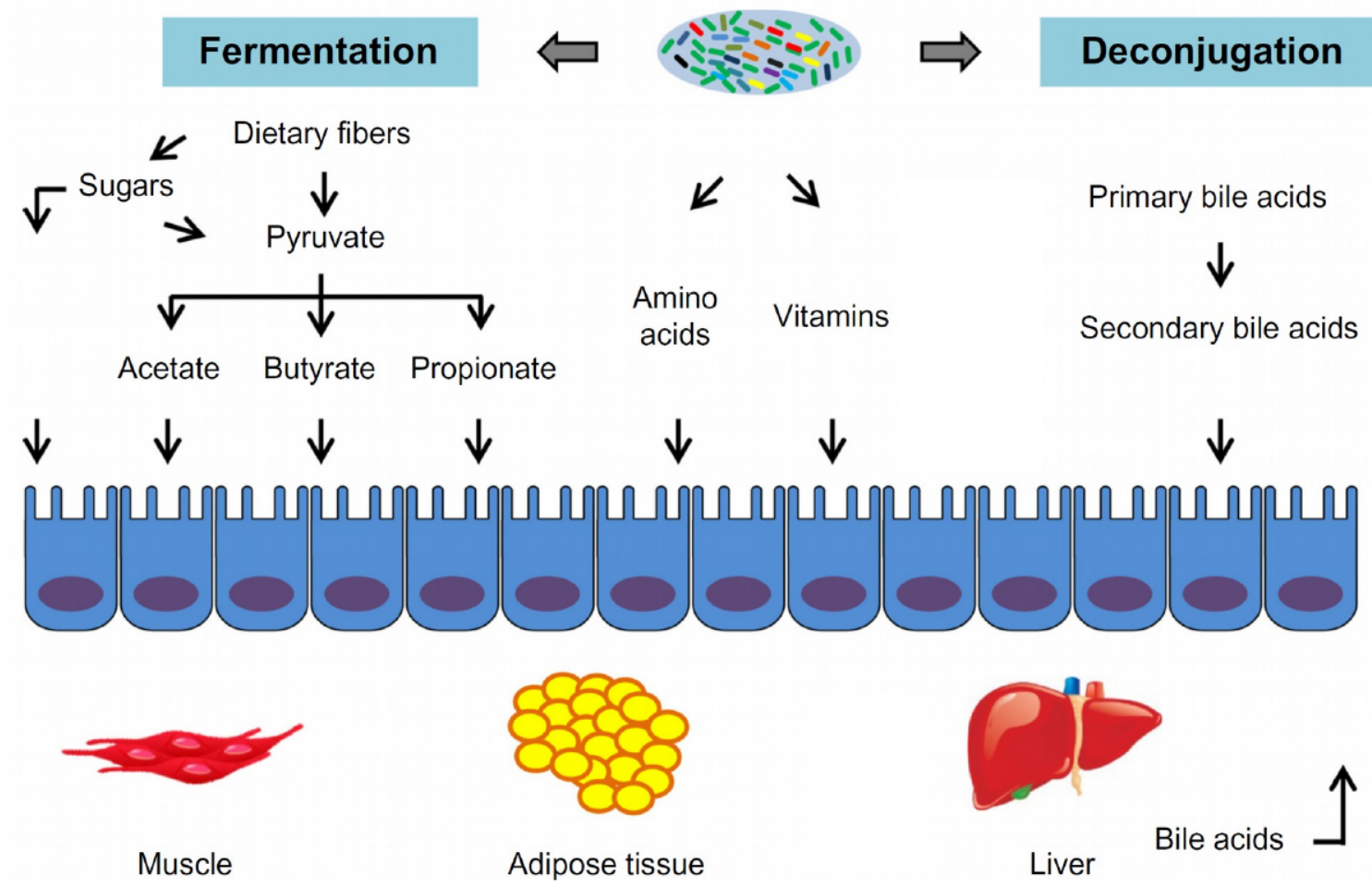

Figure I2. The gut microbiota is involved in host metabolism processes such as anaerobic fermentation, vitamin biosynthesis, and bile acid metabolism. The polysaccharides, that form part of the dietary fibers, can be degraded by the gut microbiota via anaerobic fermentation. The main products are short-chain fatty acids: acetate, propionate, and butyrate. These metabolites can be absorbed by the gut epithelial cells and then transferred to other tissue cells, including adipocytes and hepatocytes. The microbial cells can also synthesize amino acids and vitamins (mainly B and K). On the other hand, conjugated bile acids are synthesized in the liver and secreted into the intestinal tract through the duodenum. Most of these primary bile acids can be deconjugated by the gut microbiota in the intestine and be reabsorbed by the liver (38). Adapted by permission from Macmillan Publishers Ltd: Nature [Ji, Nielsen. 2016], copyright (2016).

These studies show the importance of the intestinal microbiota for the host physiology and metabolism. Consequently, changes in microbiota composition that affect metabolism could lead to health problems of the host. Some of the diseases that have been associated with alteration in metabolism (Inflammatory Bowel Disease, etc.) will be discussed in later sections. 


\subsubsection{Intestinal microbiota-brain axis}

Recently, there has been an increasing interest in studying the effects of the intestinal microbiota on the brain's physiological, behavioral and cognitive functions. Although the exact mechanism of gut microbiota-brain axis has not yet been fully understood and clarified, the evidence from animals and human studies has shown that the gut microbiota can play an important role in brain behavior and cognitive development by producing hormones, immune factors, and metabolites (39).

A study published in 2011 by Diaz Heijtz et al. (40) showed that germ-free (GF) mice have increased motor activity and less anxiety-like behavior compared to specific pathogen-free mice (SPF). When adult GF mice were incorporated into the SPF environment, its reduced anxiety-like behavior did not change, but its offspring's anxiety behavior returned to the normal state, indicating that there was a critical time window for the influence of gut microbiota on behavior development.

The gut microbiota is also linked to the control of the levels of several signaling molecules such as brain derived neurorotrophic factor, norepinephrine, and tryptophan in different areas of the central nervous system. These observations prompted to hypothesize on the role of the microbiota in the regulation of mood and behavior, and its contribution to the pathophysiology of mood disorders (41).

As described in previous sections, some members of the intestinal microbiota have the ability to produce SCFAs. Besides being a nutrient source for colonocytes and enterocytes, SCFAs (mainly butyrate and propionate) act as histone deacetylase inhibitors that epigenetically regulate gene expression (41). Recent findings implicate epigenetic modifications in the etiology of mood disorders via chromatin remodeling and changes in histone acetylation. Additionally, results from another study demonstrated that colonic acetate crosses the blood-brain barrier and is taken up by the brain (42). Moreover, the authors of this study showed that acetate administration is associated with activation of acetyl-coenzyme A carboxylase and changes in the expression profiles of regulatory neuropeptides that favor appetite suppression, suggesting that acetate has a direct role in central appetite regulation. 
Accordingly, alterations in the intestinal microbiota composition are associated with numerous neurological and psychiatric disorders such as Multiple Sclerosis, Parkinson's disease, Alzheimer's disease, major depressive disorder and autism (43).

\subsubsection{Effect of the intestinal microbiota on maturation of the immune system}

The role of the intestinal microbiota in maturation of the immune system is expected, taking into account that the intestinal mucosa is in direct contact with the members of the microbiota, exposing the host to a high concentration of antigens. The first comparisons of GF rodents with "microbiota-colonized animals" resulted in the observation that there were gross physiological differences between GF and the SPF state (44). Importantly, the absence of commensals has profound effects on the structural and functional development of the immune system, including but not limited to defects in lymphoid tissue development within the spleen, thymus, and lymph nodes.

The effect that the intestinal microbiota can have on the maturation of the immune system has been shown in several studies that compared innate lymphoid cells (ILCs) composition and characteristics between GF and SPF mice $(45,46)$. For example, in a study published in 2009, Sanos et al. (46) showed that signals from the commensal microbiota contribute to the differentiation of a subgroup of ILCs. Besides, GF mice were shown to have a decreased number of CD4+ and CD8+ intestinal $\mathrm{T}$ cell subsets (47), indicating that the intestinal microbiota has a role in development of the adaptive immune system as well.

Although these are only few examples demonstrating the role of the intestinal microbiota in the host immune system development, there are many more described (48). Collectively, these studies demonstrate that the microbiota participates in the maturation of the immune system and suggests that specific events in association with the process of microbial colonization may be important in the development of a normal immune system in a healthy individual. Besides its role in the maturation of the immune system, microbiota has the capability to induce the immune system in order to protect the host against pathogens, which will be explained in more detail in the following section. 


\subsubsection{Protection against pathogens}

The intestinal microbiota can provide protection against pathogens, also known as colonization resistance (CR), indirectly, through induction of the immune system (49). Besides this indirect mechanism of protection, intestinal microbiota can provide direct protection by competing for space and nutrients with intruders and by secreting inhibitory molecules (Figure I3).

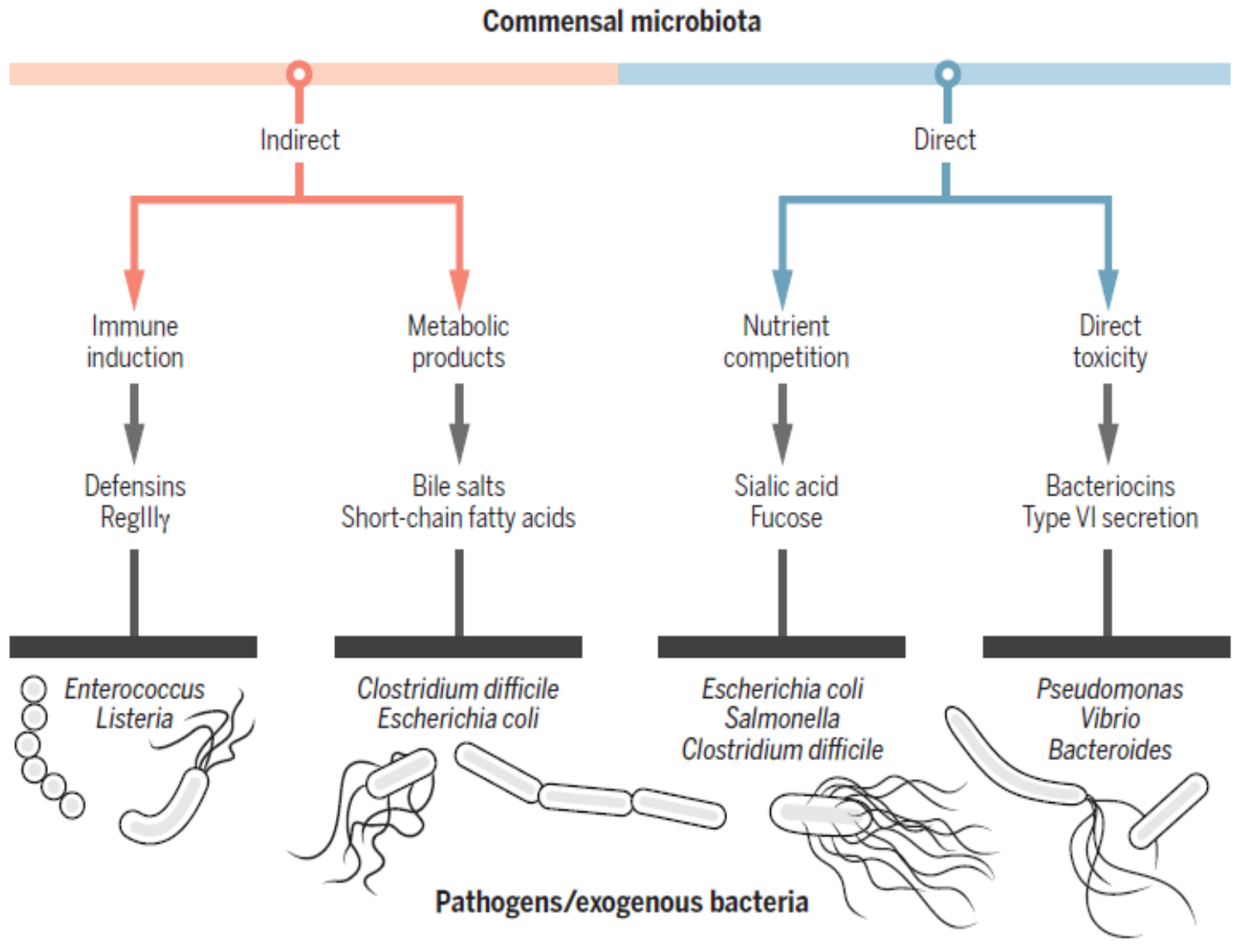

Figure I3. The intestinal commensal microbiota provides colonization resistance against a wide range of pathogens by indirect and direct mechanisms. Commensal bacteria activate innate immune defenses in the mucosa (e.g. defensins with bactericidal activity), produce or modify host-derived metabolites with inhibitory capacities (e.g. SCFA), deplete nutrients, or produce substances that are directly toxic to competing bacteria (e.g. bacteriocins) (49). Adapted from Pamer E., Science. 2016. Reprinted with permission from AAAS. 
Microbiota mediated protection against colonization with pathogens, especially against multidrug resistant Enterobacteriaceae, will be investigated in this thesis and thus, mechanisms that confer CR will be explained in detail in the following sections.

\subsection{Protection mediated through microbiota induction of the immune system}

The intestinal microbiota not only helps in the maturation of the immune system, it also induces innate and adaptive immune responses in the presence of pathogens and also promotes the maintenance of the intestinal barrier, allowing better protection against infection.

One mechanism by which commensals enhance the host immune response to pathogens is by triggering the secretion of host antimicrobial peptides. Indeed, GF mice exhibit diminished Paneth cell's expression of antimicrobial peptides RegIII $\gamma$, RegIII $\beta$ and defensin Defcr-rs-10 (50). Besides, it was shown that the microbiota plays an important role in the induction of alpha-defensin secretion by Paneth cells, which in turn controls pathogen growth and restrains commensals within the intestine (44). Moreover, in a study published in 2012, Hasegawa et al. (51) demonstrated that translocation of commensal bacteria was essential to promote the secretion of the cytokine IL-1 $\beta$ and subsequent protection against $C$. difficile intestinal colonization. This result indicates that stimulation of pro-inflammatory responses by commensal organisms can have a protective function. Besides, production of IL-22 by a subgroup of innate lymphoid cells (ILC3) is dependent on the presence of commensal Lactobacillus reuteri (52). A metabolic product of this bacterium is a ligand for a receptor present on membrane of ILC3 and activates the production of IL-22, providing CR against the fungus Candida albicans.

Besides stimulating innate immunity, intestinal microbiota can also induce the activation of the adaptive immunity. Studies using GF mice colonized with different commensal microbes have demonstrated that certain intestinal bacterial species play a major role in the differentiation of $\mathrm{T}$ cells into different subsets, including $\mathrm{T}$ helper cells and $\mathrm{T}$ regulatory cells (Tregs) (50). For example, segmented filamentous bacterium promotes the development of Th17 cells, which confer protection against Citrobacter rodentium infection (53). On the other hand, several studies have demonstrated that the 
intestinal microbiota influences B-cell development and antibody production. Kim et al. (54) demonstrated that SCFA, derived from microbiota metabolism, directly affect the differentiation of B cells into IgA-producing plasma cells. Consequently, mice receiving a diet with high levels of fiber (a major source of microbial production of SCFA) had higher numbers of intestinal IgA + B cells and were more resistant to C. rodentium intestinal colonization than those animals fed with a low-fiber diet.

\subsection{Direct protection against pathogens conferred by the intestinal microbiota}

As mentioned before, microbiota provides direct protection against pathogens through secretion of inhibitory molecules, and by competing with them for space and nutrients. Besides, commensal bacteria produce secondary metabolites that could exert a negative effect on pathogen's growth. In a recent study, Buffie et al. (27) demonstrated that secondary bile acids, produced by dehydroxylation of primary bile acids as a result of $C$. scindens metabolism, confer protection against $C$. difficile in a mouse model of infection by directly inhibiting the growth of the pathogen. In addition, some microbes produce antimicrobial proteins that can target pathogens. Some commensal Enterobacteriaceae, including the probiotic Escherichia coli Nissle, secrete small antimicrobial peptides called microcins (bacteriocins of less than $10 \mathrm{kDa}$ ) (55). In a study published in 2016, Sassone-Corsi et al. (56) demonstrated that the probiotic strain E. coli Nissle 1917 limits the growth of invasive E. coli strain through production of microcins. Besides, the intestinal microbiota could confer protection through the production of SCFA. Indeed, in vitro, SCFA markedly suppress the growth of the pathogenic E. coli strain O157:H7 (50). SCFA can also influence the expression of virulence factors. For example, butyrate and propionate, both present in high concentrations in the large intestine, downregulate the expression of genes encoded in Salmonella typhimurium pathogenicity island 1 (SP1), required by this pathogen for invasion of intestinal epithelial cells.

Another group of mechanisms by which commensal bacteria provide CR to pathogens include direct interaction by competing for the same niche (Figure I3) (57). Some beneficial microbes acquire similar nutrients as pathogens, often more efficiently, thus hindering the replication and colonization of the infectious agent. For instance, 
commensal E. coli strain HS and the probiotic strain E. coli Nissle 1917 occupy a unique nutritional niche in the mouse gut (58). E. coli HS consumes 6 sugars and E. coli Nissle 1917 uses a complementary yet divergent set of 7 sugars to colonize, thus establishing a nutritional basis to occupy distinct niches in the mouse intestine. Together these 2 commensals use the 5 sugars previously determined to be the most important for colonization of pathogenic E. coli strain, thereby impeding its successful colonization of the gut. 


\subsection{Dysbiosis of the intestinal microbiota}

\subsubsection{Definition of the dysbiosis}

Dysbiosis is a term that explains imbalance in the composition of the resident commensal communities relative to the community found in healthy individuals (59). It is characterized by loss of beneficial microbial organisms, expansion of pathobionts (members of microbiota with potential to contribute to the disease development) or other harmful microorganisms, and loss of overall microbial diversity. Factors that could promote alteration of the structure of the intestinal microbiota include diet, infection, or medical interventions (such as antibiotics), and are explained in detail in the following section.

\subsubsection{Factors that promote microbiota dysbiosis}

\subsubsection{Diet as a promoter of microbiota dysbiosis}

Association between the diet and the intestinal microbiota dysbiosis has been investigated in various studies. In a study published in 2016, Desai et al. (60) demonstrated that dietary-fiber deprivation results in clear shift in microbiota composition reflected in the decreased abundances of fiber-degrading bacteria. Importantly, during fiber deficiency, the gut microbiota resorts to host-secreted mucus glycoproteins as a nutrient source, leading to erosion of the colonic mucus barrier and high-levels of colonization with the pathogen C. rodentium. In another study, Agus et al. (61) showed that Western diet (high-fat, high-sugar diet) produced dysbiosis of the intestinal microbiota towards higher abundances of the Proteobacteria phylum and decrease in the Firmicutes phylum. Moreover, the Western diet microbiota favored colonization of adherent-invasive E. coli strain, especially in the mucus layer. The authors also observed that mice fed on Western diet become more susceptible to induced colitis, probably through decrease in abundances of protective SCFA producing bacteria, suggesting that Western diet could aggravate the inflammatory process. 


\subsubsection{Inflammation as a promoter of microbiota dysbiosis}

In a study published in 2007, Lupp et al. (62) demonstrated that the hostderived inflammation results in dysbiosis of the colonic microbial community. Infection of mice with $C$. rodentium produced inflammation, which resulted in clear reduction of the total numbers of commensal bacteria. In contrast, colonization with an enteric pathogen that does not induce inflammation (Campylobacter jejuni) failed to produce similar changes. Moreover, the authors demonstrated that inflammation produced the bloom of Enterobacteriaceae family. Interestingly, some pathogens, such as Salmonella enterica serovar Typhimurium have the ability to exploit host's inflammatory immune responses for its own benefit. In a study published by Stecher et al. (63), authors demonstrated that $S$. typhimurium overcomes $\mathrm{CR}$ by inducing inflammation and subsequent dysbiosis of the intestinal microbiota. On the other hand, a non-virulent strain of $S$. typhimurium was not able to produce inflammation and colonize the murine intestine. Importantly, colonization of mice with virulent $S$. typhimurium resulted in domination of Proteobacteria, among which E. coli reached high levels, suggesting that other bacterial species closely related to S. typhimurium may also be able to benefit from the $S$. typhimurium-triggered inflammation.

\subsubsection{Antibiotic treatment as a promoter of microbiota dysbiosis}

Antibiotics are widely used as antimicrobial agents to treat bacterial infections caused by pathogenic microorganisms. However, antibiotics (even narrow-spectrum ones) do not only affect pathogens, but also commensal intestinal microbial communities. In a study published recently, Panda et al. (64) investigated the short-term effects of fluoroquinolones and beta-lactams on the microbiota composition. Authors shown that both antibiotic treatments significantly decreased microbial diversity. Moreover, fluoroquinolones, as well as beta-lactams, caused a decrease of Firmicutes phylum and an increase of the Bacteroidetes phylum. In another study published by Ubeda et al. (65), authors described microbiota dysbiosis in mice as a result of treatment with ampicillin or vancomycin. All mice receiving ampicillin had increased abundances of Streptococcaceae family, and decreased levels of other bacterial families present in 
untreated animals. Moreover, mice treated with ampicillin had reduced bacterial density both in ileum and cecum. On the other hand, vancomycin treatment resulted in the expansion of Enterobacteriaceae family in 4 out of 6 mice. Besides, treatment with vancomycin, an antibiotic that selectively targets Gram-positive bacteria, eliminated the Gram-negative phylum Bacteroidetes from the cecum. These results were later confirmed in a study published by Isaac et al. (66). Authors found that vancomycin treatment greatly reduced the levels of the Bacteroidetes phylum, while the Proteobacteria and Fusobacteria phyla underwent a drastic expansion. Moreover, Isaac et al. demonstrated that the changes in the microbiota composition as a consequence of vancomycin treatment persisted several months after the cessation of the treatment. Other authors also pointed to the long-term effects of antibiotic treatment on the microbiota composition. In a study published in 2015, Rashid et al. (67) demonstrated the long-term effects of ciprofloxacin and clindamycin on the microbiota composition. Authors shown that exposure to ciprofloxacin or clindamycin had a strong effect on the diversity of the microbiome. The interval needed for the microbiota to be normalized after ciprofloxacin or clindamycin treatment was different for various bacterial species. Overall, changes in the microbiota composition could be observed up to 2 months post-treatment, although alterations in abundances of few members of the intestinal microbiota could be detected up to 4 months after the treatment for both antibiotics.

Recently, there has been an increasing interest in analyzing not only the effects of different antibiotic treatments on microbiota composition, but also it's effects on functional diversity of the microbiota. Perez-Cobas et al. (68) conducted the first comparative omic (word that implies set of metabolomics, metagenomics, metatranscriptomics and metaproteomics) investigation of gut microbial communities in fecal samples taken from a patient subjected to beta-lactam therapy. Authors demonstrated that beta-lactam treatment produces alteration of metabolic and protein profiles, besides changes in the microbiota composition. 


\subsubsection{The effect of the dysbiosis on the host's health}

\subsubsection{Dysbiosis and non-infectious diseases}

The adverse effects of dysbiosis on host health have long been appreciated. Increasing clinical evidence links dysbiosis with various immune, metabolic and neurological disorders in both intestinal and extra-intestinal sites. Intensively studied examples for which dysbiosis of the intestinal microbiota has been described include Inflammatory Bowel Disease (IBD) (69), diabetes (70), asthma (71), and even autism (72).

IBD is a collective name for chronic inflammatory disorders of the GIT, of which Crohn's disease (CD) and ulcerative colitis (UC) are the most prevalent forms (28). In a study published in 2011, Joossens et al. (73) found that 5 bacterial species defined the dysbiosis observed in CD patients. Moreover, higher levels of one of these species (Faecalibacter prausnitzii) have been associated with lower disease remission in $\mathrm{CD}$ patients after treatment. Interestingly, a recent study observed a reduction of Roseburia hominis and F. prausnitzii in fecal samples of UC patients, and both species showed an inverse correlation with disease activity (69).

Necrotizing enterocolitis is a diseases characterized by gut inflammation that is most frequent in premature newborns (74). It affects up to $10 \%$ of infants with a birth weight below $1500 \mathrm{~g}$. Mortality can be as high as $30 \%$. The gut microbiota dysbiosis has been associated with the onset of the necrotizing enterocolitis, since a rise in Proteobacteria and a concomitant fall of Firmicutes levels has been found to precede the onset of the disease.

A growing body of evidence has indicated that alterations of the gut microbiome may affect central nervous system (CNS). In a study published by Lyte et al. (75) authors have shown that infection with pathogen C. rodentium significantly increased anxietylike behavior in mice.

Besides the role of the microbiota dysbiosis in non-infectious diseases, recent studies have shown an association between microbiota dysbiosis and infections produced by antibiotic resistant pathogens, such as VRE (76) or $C$. difficile (24). The role of the 
microbiota dysbiosis in colonization of the intestinal tract with pathogens will be described in detail in following section.

\subsubsection{Dysbiosis and colonization of the intestinal tract by pathogens}

As discussed in previous sections, the intestinal microbiota has a crucial role in the protection against pathogens. Thus, it is not surprising that altered representation of the microbiota members, especially as a result of the antibiotic treatment, is associated with the loss of $\mathrm{CR}$ and, consequently, intestinal tract colonization by pathogens (Figure I4).

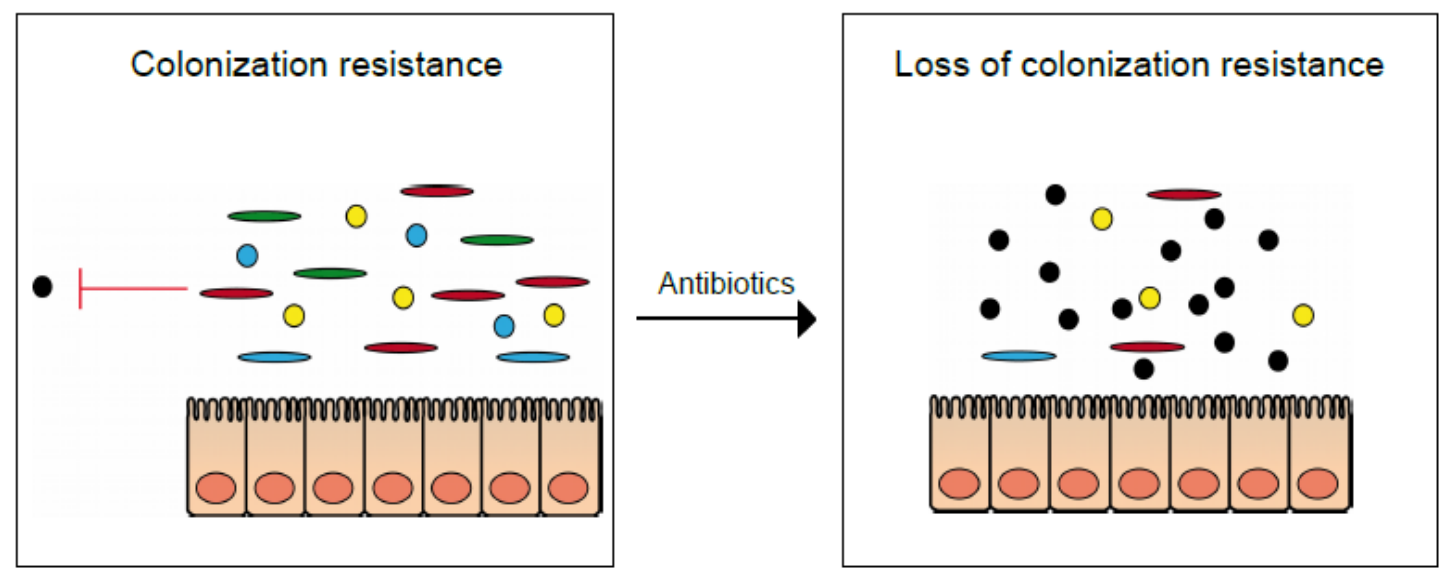

Figure I4. The effect of the antibiotic treatment on microbiota composition and colonization resistance against pathogens. Antibiotics can decrease overall bacterial diversity which may promote intestinal colonization by multidrug resistant pathogens (black circles).

Early studies by Freter, Bohnhoff, and their colleagues (23) roughly 50 years ago demonstrated that antibiotic administration enhanced infection with Vibrio cholerae or S. typhimurium by eliminating obligate anaerobic bacteria. Recently, clindamycin has been shown to have a large negative impact on the intestinal microbiota as seen by reduced resistance to colonization by pathogens, leading to a high risk for pseudomembranous colitis due to $C$. difficile overgrowth in humans (77). In another study, Buffie et al. (24) demonstrated that inoculation of clindamycin-treated mice with C. difficile spores results in rapid development of diarrhea and colitis, with a 4- to 5-day period of profound weight loss and associated 40 to $50 \%$ mortality rate. In contrast, mice 
that did not receive the antibiotic were resistant to intestinal colonization by this pathogen.

It is important to point out that in order to colonize the intestine during the antibiotic treatment, the pathogenic bacteria have to be resistant to the antibiotic. For this reason, besides $C$. difficile, another pathogen that frequently colonizes the gastrointestinal tract during antibiotic therapy is VRE, which is multidrug resistant. Indeed, it has been shown that the loss of CR during the vancomycin treatment allows colonization of the intestinal tract of mice with VRE (65). Members of the family Enterobacteriaceae represent another group of pathogens with resistances to many antibiotics, and that often colonize the human gut as a result of loss of CR due to antibiotic treatment (78). The association between microbiota dysbiosis and the colonization of the intestine by these pathogens, as well as the factors that promote their colonization, are the object of investigation in this thesis and are explained in more detail in the following section.

\subsection{Gut colonization by multidrug resistant Enterobacteriaceae (MRE)}

The members of family Enterobacteriaceae, such as E. coli and Klebsiella pneumoniae, are among the leading causes of morbidity and mortality worldwide (56). In the past decades, Enterobacteriaceae have acquired multiple antibiotic resistances (79). Increasing number of antibiotics to which isolated Enterobacteriaceae strains express resistance led to the definition of multidrug resistant Enterobacteriaceae (MRE). MRE are defined by Magiorakos et al. (80) as those Enterobacteriaceae non-susceptible to at least 1 agent in 3 or more antimicrobial categories, not taking into account those antibiotics for which there is an intrinsic resistance (Table I1). 
Role of intestinal dysbiosis on gut colonization by bacterial pathogens

Table I1. Antimicrobial categories and agents used to define MRE. Reprinted from Multidrug-resistant, extensively drug-resistant and pandrug-resistant bacteria: An international expert proposal for interim standard definitions for acquired resistance, Magiorakos AP. et al., Clinical Microbiology and Infection, 268-281, Copyright (2011), with permission from Elsevier.

\begin{tabular}{|c|c|c|}
\hline $\begin{array}{l}\text { Antimicrobial } \\
\text { category }^{\mathrm{a}}\end{array}$ & Antimicrobial agent & Species with intrinsic resistance \\
\hline \multirow[t]{4}{*}{ Aminoglycosides } & Gentamicin & $\begin{array}{l}\text { Providencia rettgeri (P. rettgeri), } \\
\text { Providencia stuartii (P. stuartii) }\end{array}$ \\
\hline & Tobramycin & P. rettgeri, $P$. stuartii \\
\hline & Amikacin & \\
\hline & Netilmicin & P. rettgeri, P. stuartii \\
\hline \multirow{2}{*}{$\begin{array}{l}\text { Antipseudomonal } \\
\text { penicillins }+ \text { beta- } \\
\text { lactamase inhibitors }\end{array}$} & $\begin{array}{l}\text { Ticarcillin-clavulanic } \\
\text { acid }\end{array}$ & $\begin{array}{l}\text { Escherichia hermannii (E. } \\
\text { hermanii) }\end{array}$ \\
\hline & Piperacillin-tazobactam & E. hermanii \\
\hline \multirow[t]{4}{*}{ Carbapenems } & Ertapenem & \\
\hline & Imipenem & \\
\hline & Meropenem & \\
\hline & Doripenem & \\
\hline \multirow[t]{2}{*}{$\begin{array}{l}\text { Non-extended spectrum } \\
\text { cephalosporins; 1st and } \\
\text { 2nd generation } \\
\text { cephalosporins }\end{array}$} & Cefazolin & $\begin{array}{l}\text { Citrobacter freundii (C. freundii), } \\
\text { Enterobacter aerogenes (E. } \\
\text { aerogenes), Enterobacter cloacae } \\
\text { (E. cloacae), Hafnia alvei (H. } \\
\text { alvei), Morganella morganii (M. } \\
\text { morganii), Proteus penneri (P. } \\
\text { penneri), Proteus vulgaris (P. } \\
\text { vulgaris), P. rettgeri, P. stuartii, } \\
\text { Serratia marcescens (S. } \\
\text { marcescens) }\end{array}$ \\
\hline & Cefuroxime & $\begin{array}{l}\text { M. morganii, P. penneri, P. vulgaris, } \\
\text { S. marcescens }\end{array}$ \\
\hline \multirow{3}{*}{$\begin{array}{l}\text { Extended-spectrum } \\
\text { cephalosporins; 3rd and } \\
\text { 4th generation } \\
\text { cephalosporins }\end{array}$} & $\begin{array}{l}\text { Cefotaxime or } \\
\text { ceftriaxone }\end{array}$ & \\
\hline & \begin{tabular}{|l|} 
Ceftazidime \\
\end{tabular} & \\
\hline & Cefepime & \\
\hline \multirow[t]{2}{*}{ Cephamycins } & Cefoxitin & $\begin{array}{l}\text { C. freundii, E. aerogenes, } E \text {. } \\
\text { cloacae, } H \text {. alvei }\end{array}$ \\
\hline & Cefotetan & $\begin{array}{l}\text { C. freundii, E. aerogenes, } E \text {. } \\
\text { cloacae, } H \text {. alvei }\end{array}$ \\
\hline
\end{tabular}


Introduction

\begin{tabular}{|c|c|c|}
\hline $\begin{array}{l}\text { Antimicrobial } \\
\text { category }^{\mathrm{a}}\end{array}$ & Antimicrobial agent & Species with intrinsic resistance \\
\hline Fluoroquinolones & Ciprofloxacin & \\
\hline $\begin{array}{l}\text { Folate pathway } \\
\text { inhibitors }\end{array}$ & $\begin{array}{l}\text { Trimethoprim- } \\
\text { sulphamethoxazole }\end{array}$ & \\
\hline Glycylcyclines & Tigecycline & $\begin{array}{l}\text { M. morganii, Proteus mirabilis }(P . \\
\text { mirabilis), } P \text {. penneri, } P \text {. vulgaris, } P \text {. } \\
\text { rettgeri, } P \text {. stuartii }\end{array}$ \\
\hline Monobactams & Aztreonam & \\
\hline Penicillins & Ampicillin & $\begin{array}{l}\text { Citrobacter koseri (C. koseri), } C . \\
\text { freundii, E. aerogenes, E. cloacae, } \\
\text { E. hermanii, H. alvei, Klebsiella } \\
\text { spp., M. morganii, P. penneri, } P . \\
\text { vulgaris, P. rettgeri, P. stuartii, } S . \\
\text { marcescens }\end{array}$ \\
\hline \multirow[t]{2}{*}{$\begin{array}{l}\text { Penicillins }+ \text { beta- } \\
\text { lactamase inhibitors }\end{array}$} & $\begin{array}{l}\text { Amoxicillin-clavulanic } \\
\text { acid }\end{array}$ & $\begin{array}{l}\text { C. freundii, E. aerogenes, } E \text {. } \\
\text { cloacae, } H . \text { alvei, } M . \text { morganii, } P \text {. } \\
\text { rettgeri, } P \text {. stuartii, S. marcescens }\end{array}$ \\
\hline & Ampicillin-sulbactam & $\begin{array}{l}\text { C. freundii, C. koseri, E. aerogenes, } \\
\text { E. cloacae, H. alvei, P. rettgeri, S. } \\
\text { marcescens }\end{array}$ \\
\hline Phenicols & Chloramphenicol & \\
\hline Phosphonic acids & Fosfomycin & \\
\hline Polymyxins & Colistin & $\begin{array}{l}\text { M. morganii, } P \text {. mirabilis, } P . \\
\text { penneri, } P . \text { vulgaris, } P . \text { rettgeri, } P \text {. } \\
\text { stuartii, } S \text {. marcescens }\end{array}$ \\
\hline \multirow[t]{3}{*}{ Tetracyclines } & Tetracycline & $\begin{array}{l}\text { M. morganii, } P \text {. mirabilis, } P \text {. } \\
\text { penneri, } P . \text { vulgaris, } P \text {. rettgeri, } P \text {. } \\
\text { stuartii }\end{array}$ \\
\hline & Doxycycline & $\begin{array}{l}\text { M. morganii, P. penneri, } P \text {. vulgaris, } \\
\text { P. rettgeri, P. stuartii }\end{array}$ \\
\hline & Minocycline & $\begin{array}{l}\text { M. morganii, P. penneri, } P \text {. vulgaris, } \\
\text { P. rettgeri, P. stuartii }\end{array}$ \\
\hline
\end{tabular}

${ }^{a}$ When a species has intrinsic resistance to an antimicrobial agent or to the whole category, that agent or category should not be counted when calculating the number of agents or categories to which the bacterial isolate is non-susceptible.

Nosocomial infections caused by MRE represent a major threat world-wide (81). Indeed, the last report of the European Center for Disease Prevention and Control 
from 2015 revealed that during one year 21,871 strains of $K$. pneumoniae displaying combined resistance to third-generation cephalosporins, fluoroquinolones and aminoglycosides were isolated in hospitals in the European Union (Figure I5) (82). Moreover, the percentage of strains with combined resistance increased significantly from $17.7 \%$ in 2012 to $18.6 \%$ in 2015 . The last resort antibiotics, mostly prescribed offlabel, are 2 revived antimicrobials of the 1970s to 1980s, colistin and fosfomycin, as well as tigecycline, which in combination with an aminoglycoside have shown promising efficacy both in vitro and in vivo in the critically ill host. Nevertheless, Enterobacteriaceae strains resistant to these antibiotics have already been found.

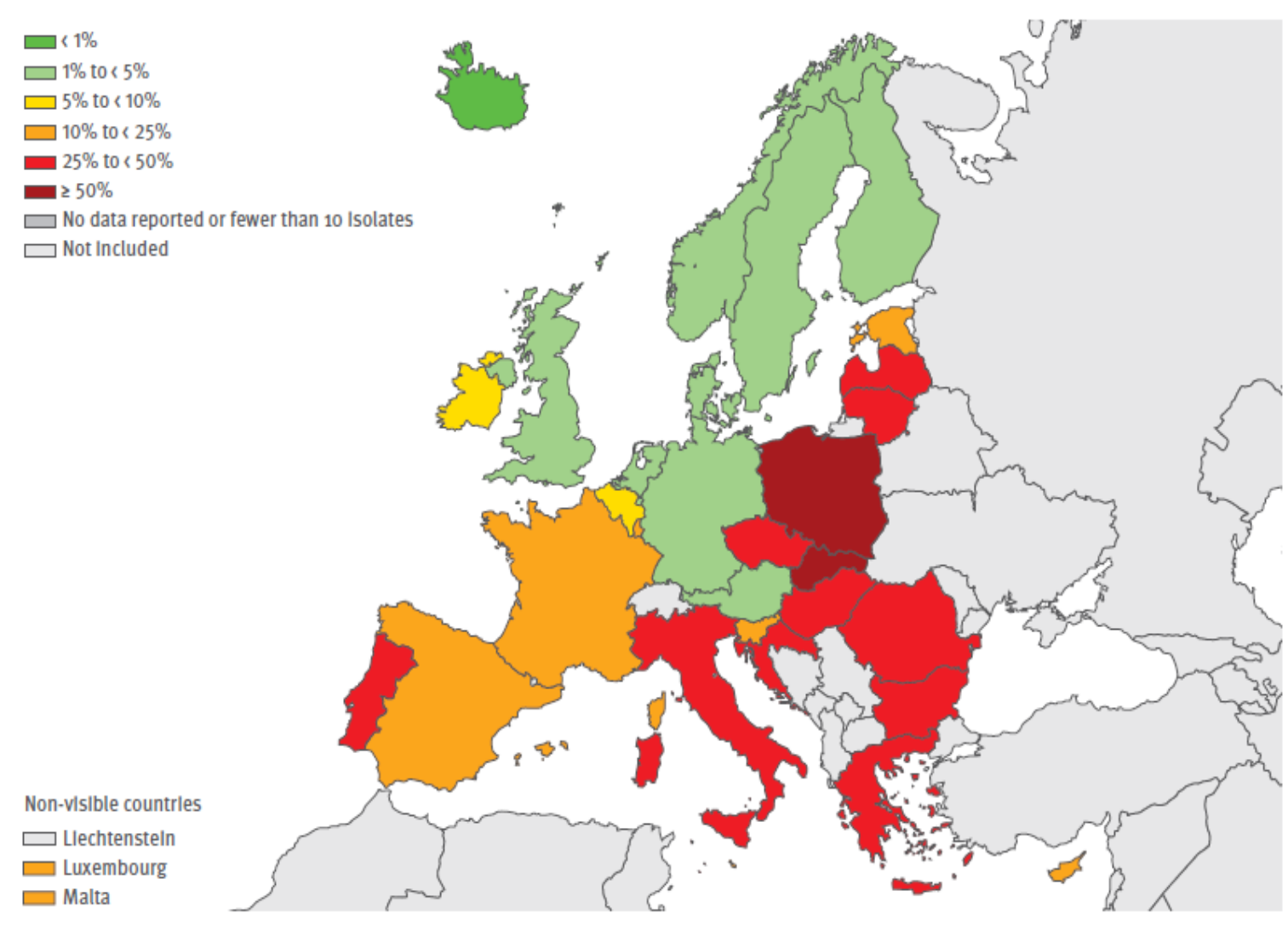

Figure I5. Percentage (\%) of invasive isolates of $K$. pneumoniae with combined resistance to fluoroquinolones, third-generation cephalosporins and aminoglycosides, by country in $\mathbf{2 0 1 5}$. Adapted from European Center for Disease Prevention and Control, 2017.

Infections with MRE usually begin by the colonization of the intestinal tract. Moreover, once established, MRE can reach high numbers in the intestine, which promotes their dissemination into the bloodstream where they can be a real threat for 
patient's life (83). Importantly, in a study published recently, Woerther et al. (83) showed that carriers with the highest level of extended spectrum beta-lactamase (ESBL) producing Enterobacteriaceae were also those with the highest risk of bacteremia produced by these pathogens. Besides, high levels of colonization promote transmission of the pathogen among patients (84). Despite the importance, to this date, there is no study investigating the risk factors that could be associated with MRE levels of colonization in hospitalized patients.

Importantly, a healthy intact microbiota provides $\mathrm{CR}$ against colonization with MRE. For example, mice are protected against intestinal colonization by MRE unless their microbiota is altered with antibiotics (85). Several studies have tried to identify factors associated with fecal colonization with antibiotic-resistant Enterobacteriaceae in humans (presence of the strain in feces, no matter the level of colonization). For example, in a study published in 2015, Torres-Gonzalez et al. (86) identified hematologic malignancy as one of the risk factors for carbapenem-resistant Enterobacteriaceae (CRE) fecal carriage. The authors point to the fact that hematologic patients are subjected to constant readmission to health care facilities, and administration of broad-spectrum antibiotics and chemotherapy agents that may disrupt the gastrointestinal microbiota, thus rendering them prone to colonization by CRE. In the same study, authors reported carbapenem treatment as one of the risk factors for CRE fecal carriage. In another study, Mittal et al. (78) identified the use of aminoglycosides as clinical variable associated with CRE colonization. These results corroborate those obtained in studies performed in mice. Caballero et al. (85) demonstrated that ampicillin treatment renders mice highly susceptible to colonization with $K$. pneumoniae. In another study, Hoyen et al. (87) studied the effect of different antibiotic treatments on colonization by ESBLproducing $K$. pneumoniae in mice. The authors found that antibiotics with minimal activity against intestinal anaerobes (i.e., cefepime, aztreonam, and levofloxacin) did not promote colonization by ESBL-producing $K$. pneumoniae, whereas an anti-anaerobic agent (i.e., clindamycin) promoted high-density colonization. These results support the hypothesis that the indigenous anaerobic microbiota inhibit colonization with antibioticresistant Enterobacteriaceae, such as ESBL-producing $K$. pneumoniae, although the 
bacterial species that provide CR against members of Enterobacteriaceae family remain unknown.

Despite the importance of MRE, little is known about clinical factors associated with MRE colonization levels or which members of the microbiota could prevent colonization and subsequent infection with MRE. One of the objectives of this thesis is to respond to these questions.

\subsection{Dysbiosis as a consequence of colonization with intestinal pathogens}

The protective capacity of the gut microbiota against pathogen intestinal colonization is well documented and explained in previous sections. As indicated above, bacterial pathogens such as MRE do not successfully colonize the intestinal tract unless the microbiota is altered, mainly by administration of antibiotics. Nevertheless, there are some exceptions to this. For example, S. typhimurium is able to colonize the murine gut without previous exposure to antibiotics (88). After initial colonization of the gut, Salmonella induces inflammation, which alters the microbiota composition and prevents suppression of Salmonella by the microbiota. Thus in this case, external factors such as antibiotics are not required for altering the microbiota in order for the pathogen to colonize the intestine, but rather the dysbiosis is induced by the pathogen, and subsequently enhances pathogen intestinal colonization (63). Importantly, Salmonella induced dysbiosis promotes the bloom of members of family Enterobacteriaceae (e.g. E. coli), suggesting that conditions created as a result of infections with some pathogens could favor the expansion of other putative pathogenic bacteria.

Thus, in some cases dysbiosis of the intestinal microbiota opens the way to colonization by pathogens such as MRE. Yet, in case of some other pathogens, dysbiosis occurs after the initial pathogen colonization. Although the role of the microbiota dysbiosis is clear for some diseases, for others it remains unknown. Epizootic rabbit enteropathy is one of the diseases for which the role of the microbiota dysbiosis remains unclear, and will be explained in detail in the following section. 


\subsubsection{Epizootic rabbit enteropathy}

Epizootic rabbit enteropathy (ERE) is a severe gastrointestinal disease with 30$80 \%$ of mortality, and with a particular incidence in the post-weaning period (89). Since it was first identified ERE has become a serious concern for rabbit breeders in most European countries, and causes significant economic losses in rabbit farms. Moreover, there have been reports of ERE cases in South America and Turkey recently $(90,91)$. This pathology is characterized by a decrease in food intake followed by distended abdomen, emission of small quantities of watery diarrhea, appearance of the mucus in feces, and decrease in daily weight gain (92). Pathological changes at the intestinal level include distension of the stomach and/or duodenum, presence of liquid and gaseous content in the intestine and impaction, especially in cecum. All these changes are consistent with a prolonged paresis of the intestinal transport. Indeed, some authors have reported that intestinal ligation reproduces ERE symptoms (89). Disease could be reproduced by contact between healthy and sick animals or healthy animals and contaminated breeding material, which is why it is suspected that ERE is a contagious disease and therefore that a pathogenic agent is involved in the development of this intestinal pathology. However, no causative agent has been identified for the moment.

In 2005 Licois et al. (92) reproduced the disease in healthy animals by administrating an inoculum obtained from rabbits affected by ERE. This inoculum was obtained by mixing contents of the small intestine, cecum and colon of sick animals, and was named TEC. Thus, at least part of the Koch's postulate was respected, although the authors were not able to isolate and identify the causative agent of the disease.

In another study published in 2007, Szalo et al. (93) fractioned one of the reference TEC inoculums on a discontinuous sucrose gradient. Different fractions were used for infection of healthy rabbits. Fractionation of the inoculum has allowed the separation of microorganisms according to type, size, morphology and density. In such conditions, small entities, such as viruses and toxins, are isolated in the upper fraction, large entities, such as parasites, are isolated in the pellet and lower fraction, and bacteria were isolated in the middle fraction. The authors demonstrated that the etiological agent of ERE was present in the middle fraction, suggesting that the ERE etiological agent may 
be of bacterial origin, although authors were not able to identify which specific species is the cause of the disease.

Various authors have pointed to the possible role of $C$. perfringens in ERE. Marlier and coworkers (94) detected high fecal counts of $C$. perfringens in the digestive contents of $80 \%$ of the ERE field cases. On the other hand, Romero et al. (95) showed that high counts of $C$. perfringens in the hindgut are associated with clinical symptoms of ERE and mortality. Nevertheless, both authors point to the fact that these associations could be the consequence of the disease rather than the cause. In fact, no experimental reproduction of ERE has been obtained after inoculation of rabbits with strains of $C$. perfringens. Still, the observed expansion of $C$. perfringens could play a role, if not in the initiation of the disease, in the subsequent exacerbation of the symptomatology and mortality rate. Moreover, Romero et al. (95) showed that conditions that were associated with a decrease in $C$. perfringens fecal counts (delayed weaning and/or diet with increased fiber content) were also associated with a decrease in mortality rate due to ERE.

As mentioned before, ERE has been associated with intestinal dysbiosis. A recent study published by Bauerl et al. (89) described remarkable dysbiosis and reduced taxonomic diversity in the cecal microbiota of animals with ERE. Quantitative and qualitative differences between healthy and ERE rabbits, such as the abundance of Verrucomicrobiae, particularly the genus Akkermansia, were described. Akkermansia muciniphila is a typical intestinal anaerobe frequently associated to human healthy mucosa. Its high prevalence in ERE cecal samples could be related to their mucin scavenger character since, as indicated above, one of the ERE symptoms is the intestinal mucus secretion. On the other hand, Ruminococcus is the most relevant genus of the Firmicutes phylum dominant in healthy individuals, that notably decreases in ERE. In addition, a number of opportunistic pathogens were found to increase in samples obtained from ERE rabbits, including members of family Enterobacteriaceae. Nevertheless, the authors pointed out that it is unclear whether the microbiota changes observed in ERE rabbits were the cause or the consequence of the disease, a key point that, as indicated in the objectives of the thesis, we will try to elucidate by studying gut microbiota dynamics before and after the initiation of ERE. In relation to this, ERE 
occurs in young rabbits during first weeks after the weaning (89), a period during which changes in microbiota structure are known to occur. The role of these changes in ERE development is unknown and will also be studied in this thesis.

\subsubsection{Restoration of the intestinal microbiota}

As mentioned in previous sections, microbiota dysbiosis can be crucial for the colonization of the intestine by pathogens. Accordingly, restoration of the intestinal microbiota could provide protection against colonization by pathogens and could also eliminate pathogens in cases colonization have already occurred. The increasing knowledge about the importance of the intestinal microbiota in maintenance of $\mathrm{CR}$ and mechanisms that underlie this phenomenon are opening a new field for possible therapeutic approaches (Figure I6). Microbiota interventions as therapeutic strategies include fecal microbiota transplant (FMT), which implies transplant of complete microbial community from a healthy donor to a recipient, or administration of probiotics (microbial species that, upon administration confer a benefit to the host) (49).
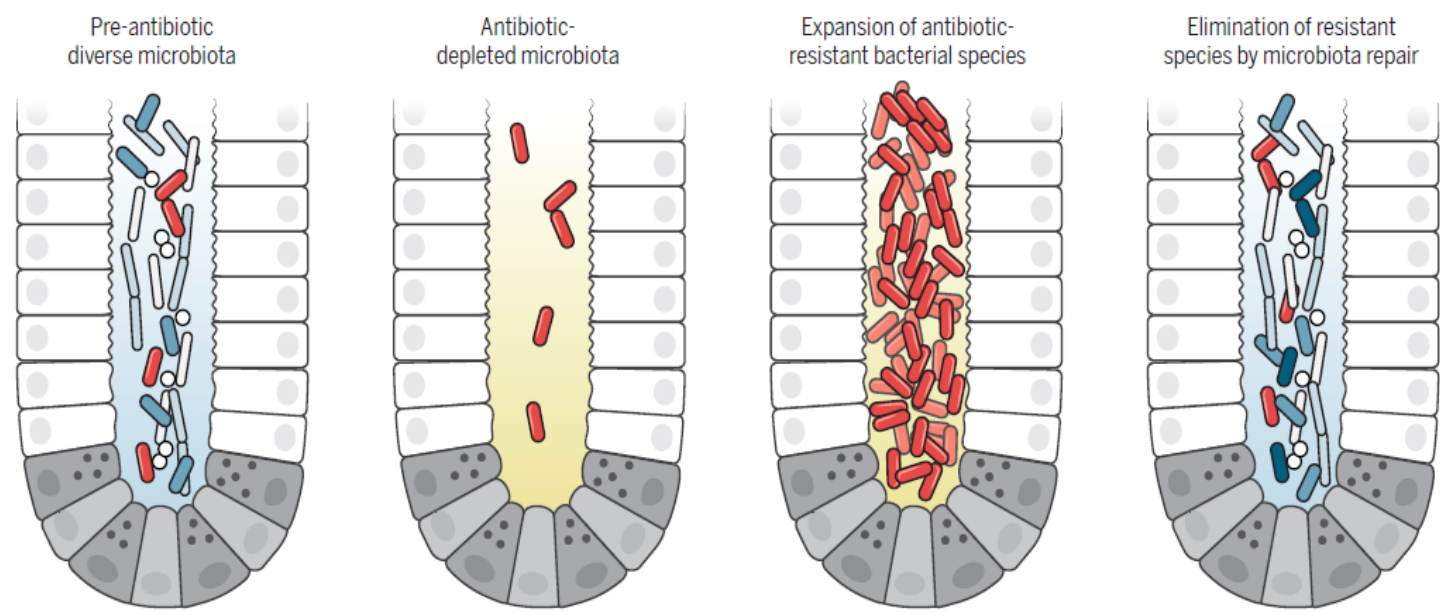

Figure I6. Restoration of the colonization resistance after the antibiotic treatment by administration of commensal bacterial species. Antibiotic-induced microbiota defects and the resulting loss of CR can be corrected by administration of commensal bacterial species. From Pamer E., Science. 2016. Reprinted with permission from AAAS.

Reconstitution of the commensal flora by adoptively transferring live bacterial populations has a long history. In China, administration of 'yellow soup', which is a 
dilute solution of human feces, was used to treat patients with intestinal infections for hundreds of years (96). Recently, fecal transplantation was used to treat recurrent $C$. difficile infections (CDI). As previously mentioned, antibiotic administration alters the microbiota and renders the host more susceptible to infection with $C$. difficile (77). Transplant of fresh or frozen feces to individuals with recurrent CDI resulted in infection resolution for approximately $90 \%$ of patients (97). More importantly, authors reported that relapses and deaths after FMT were uncommon. Recently, the first prospective randomized controlled trial evaluating duodenal infusion of donor fecal material was completed (98). The trial reported high success rates that greatly exceeded standard treatment with vancomycin (one of the antibiotic therapies used for CDI). Resolution of C. difficile-associated diarrhea after the first infusion of a solution of donor feces through a nasoduodenal tube occurred in $81 \%$ of patients, compared to $31 \%$ of the patients that received vancomycin treatment. Moreover, patients receiving fecal microbiota transplantation were reconstituted with a broad range of intestinal microbial taxa, spanning the Bacteroidetes and Firmicutes phyla. However, despite the high percentage of resolved infections, much is still unknown about why in some patients with recurrent $C$. difficile infection FMT fails. Importantly, the practice of adoptively transferring commensal bacteria to reconstitute a health-associated microbiota in individuals with disease carries risks as well as promise. Microbiota transplants may contain bacteria or virus that have uncharacterized infectious and pathological properties, and the overall effect of transplantation on the native intestinal community can be dynamic and unpredictable (96). Besides, it is even possible that a bacterium completely changes its behavior after transplantation into a new environment because of the host genotype, its diet, or the residual microbiota, turning form a beneficial bacterium into a pro-inflammatory pathobiont. Therefore, many efforts are underway to identify a mixture of beneficial bacteria, which can help to fight against enteric infections by administering small consortia of cultivable bacteria rather to transfer the whole microbiota (79).

In a study published in 2013 Petrof et al. (99) showed that a set of 33 bacteria isolated from a healthy donor was able to clear recurrent CDI. Another study identified a minimal consortium of 6 taxonomically diverse bacterial species that cleared $C$. difficile infection in a mouse model (100). In these experiments, transfer of the 6 bacterial species 
resulted in marked increase in overall microbiota diversity, which suggests that the transferred bacterial population facilitated intestinal repopulation with a diverse range of bacterial taxa. As previously mentioned, Buffie et al. (27) showed that administration of C. scindens, a commensal bacterium, is associated with resistance to CDI in patients and suppress $C$. difficile colonization in a mouse model of infection. Interestingly, several companies already have started clinical trials with "semi-standardized" donor material to treat recurrent CDI (79). Rebiotix is a company that is now planning a clinical trial for microbiota restoration therapy to prevent CDI. Moreover, they have provided evidence that administration of their product promotes clearance of VRE (79).

Importantly, the first step towards the development of probiotics is the identification of bacteria that provide $\mathrm{CR}$ against pathogens. Although for some pathogens, such as $C$. difficile, some of the key bacterial species that provide CR have been already identified, for others, such as MRE, it is unclear which members of microbiota provide protection against colonization. Identification of bacterial species that could provide $\mathrm{CR}$ against MRE is one of the objectives of this thesis. 



\section{Objectives}

The general objective of this thesis is to study the role of the microbiota dysbiosis on the colonization of intestine by bacterial pathogens. For this purpose, 3 major projects with their own specific objectives were designed:

Project 1. Microbiota dysbiosis and multidrug resistant Enterobacteriaceae (MRE) colonization and persistence in mice.

In order to study the role of microbiota dysbiosis on MRE colonization and persistence in mice, the following specific objectives were proposed:

1.1 To investigate changes in the microbiota, induced by antibiotic treatments, that promote MRE intestinal colonization and persistence.

1.2 To test if an altered microbiota can be restored to prevent MRE intestinal colonization.

1.3 To identify the specific commensal bacteria that confer protection against MRE intestinal colonization in mice.

Project 2. Microbiota dysbiosis and MRE colonization in hospitalized patients.

In order to study the role of the microbiota dysbiosis on MRE colonization in hospitalized patients, the following specific objectives were proposed:

2.1 To investigate the prevalence of MRE intestinal colonization in acute leukemia patients.

2.2 To characterize MRE strains isolated from feces of acute leukemia patients.

2.3 To identify clinical variables that promote intestinal colonization by MRE in acute leukemia patients.

2.4 To identify specific commensal bacteria that are associated with protection against MRE intestinal colonization in acute leukemia patients.

Project 3. Microbiota dysbiosis and development of Epizootic Rabbit Enteropathy (ERE)

In order to study the role of microbiota dysbiosis and development of ERE, the following specific objectives were proposed:

3.1 To define intestinal microbiota changes in rabbits suffering ERE.

3.2 To define intestinal microbiota dynamics in rabbits after weaning. 
Role of intestinal dysbiosis on gut colonization by bacterial pathogens

3.3 To define specific changes in the intestinal microbiota after weaning that could lead to ERE development.

3.4 To test if the identified microbiota changes could trigger ERE. 




\section{Material and Methods}

\subsection{Experiments setup}

\subsubsection{Mouse experimental model}

In order to determine the association between the microbiota composition and intestinal colonization with MRE various experiments using mice as a model were performed. These experiments included the treatment with different antibiotics, challenge with multidrug resistant $K$. pneumoniae (MRKP), a MRE that frequently colonized the intestinal tract of patients, and attempts to restore microbiota CR against MRKP. All experiments were approved by University of Valencia Animal Care Committee, protocol number 2015/VSC/PEA/00082, and carried out in accordance with the approved guidelines. Mice used in experiments performed as part of this thesis were 6 weeks old C57BL/6J mice obtained from Charles River, France. They were reared under SPF conditions within the animal facility at the University of Valencia. Mice had ad libitum access to food and water during the course of all experiments. Fecal samples were collected at various time points, as it will be indicated in the following sections. As previously mentioned in the Introduction, fecal samples have been used in various studies for assessing the intestinal microbiota composition. Fecal microbiota composition has been useful to identify specific changes in the microbiota that promote colonization by pathogens, including VRE (23) or $C$. difficile (24), indicating that fecal samples can provide relevant information about changes in the microbiota that contribute to intestinal pathogen colonization, which is why we decided to collect this type of samples. Immediately after collection, fecal samples were kept on ice until further processing. One part of the sample was used to assess the levels of colonization with MRKP by plate counting technique, which will be described in section 3.3.1. The other part of the sample was used for determination of microbiota composition (sections 3.4 and 3.6).

\subsubsection{Treatment of mice with antibiotics}

For assessing the effect of antibiotics on microbiota composition and MRKP colonization, mice were administered 1 of 3 different antibiotics, including ampicillin, 
vancomycin and neomycin, and a combination of these 3. All antibiotics were administered in the mouse drinking water for 1 week at following concentrations: ampicillin $0.5 \mathrm{~g} / \mathrm{l}$; vancomycin $0.5 \mathrm{~g} / \mathrm{l}$; neomycin $1 \mathrm{~g} / 1$. Treatment with combination of these 3 antibiotics was prepared by combining them at the same concentrations as used when administrating them individually. During the treatment, antibiotics were changed once, at $3^{\text {rd }} / 4^{\text {th }}$ day since the beginning of the treatment. This change was performed since we noticed that some antibiotics (i.e. ampicillin) lost part of its bactericidal activity after 4 days at room temperature. Mice were housed separately (1 mouse per cage) in experiments in which they were treated with different antibiotics to determine treatment effect on microbiota and capability of MRKP to colonize the intestine. In the second set of experiments in which the goal was to restore the intestinal microbiota by administration of fecal transplant or specific isolated commensal bacteria, mice were reared in groups of 5 per cage during the antibiotic treatment. Upon the cessation of the treatment, animals were separated and housed 1 mouse per cage.

To analyze the effect of antibiotics on the mouse intestinal microbiota, fecal samples were collected from mice treated with antibiotics on the day before the cessation of the treatment. The capability of microbiota recovery after the antibiotic treatment was assessed by collecting fecal samples 2 weeks and 4 weeks after treatment withdrawal. Mice that had not received antibiotic treatment were used as controls.

\subsubsection{MRKP challenge in mice}

Multidrug resistant $K$. pneumoniae was obtained from the Clinical Microbiology Laboratory at Memorial Sloan-Kettering Cancer Center where it was isolated from a blood sample of a hospitalized patient (85). MRKP was grown at $37^{\circ} \mathrm{C}$ in Luria-Bertani (LB) agar over night. The next morning it was resuspended in phosphate buffer saline (PBS). For infection experiments, $10^{6}$ colony-forming units (CFUs) of $K$. pneumoniae were administered to each mouse by oral gavage in a $200 \mu$ l volume. In experiments in which the capability of colonization with MRKP after different antibiotic treatments was tested, mice were infected the day before cessation of the treatment. In experiments in which mice were left to recover after antibiotic treatment or fecal transplant and specific isolated commensal bacteria were administered, infection with 
MRKP was performed 2 weeks or 4 weeks after the antibiotic treatment withdrawal (Figure M1). Fecal samples were collected before the infection and 1 day post-infection in order to determine the levels of colonization with MRKP.

A

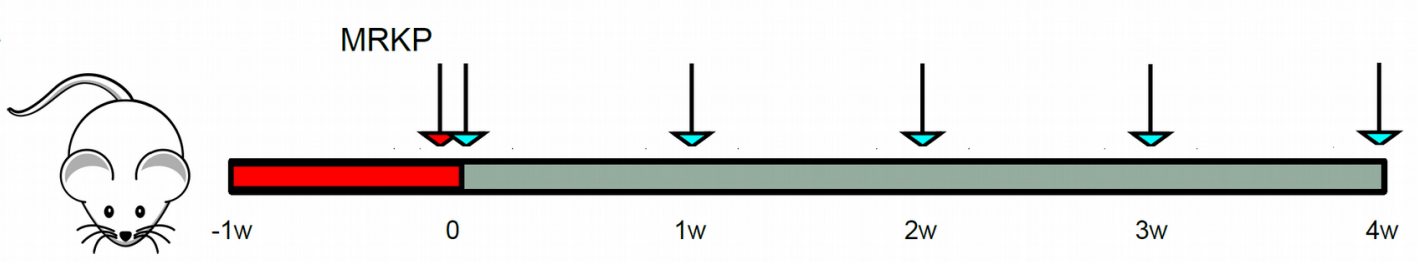

B

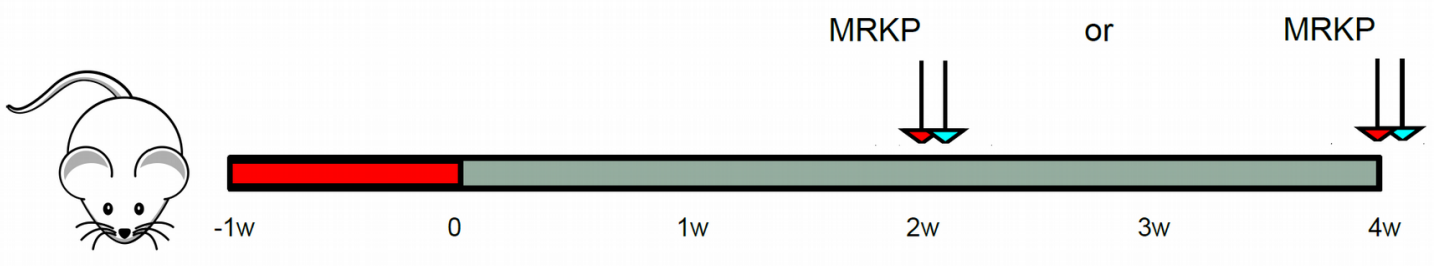

C

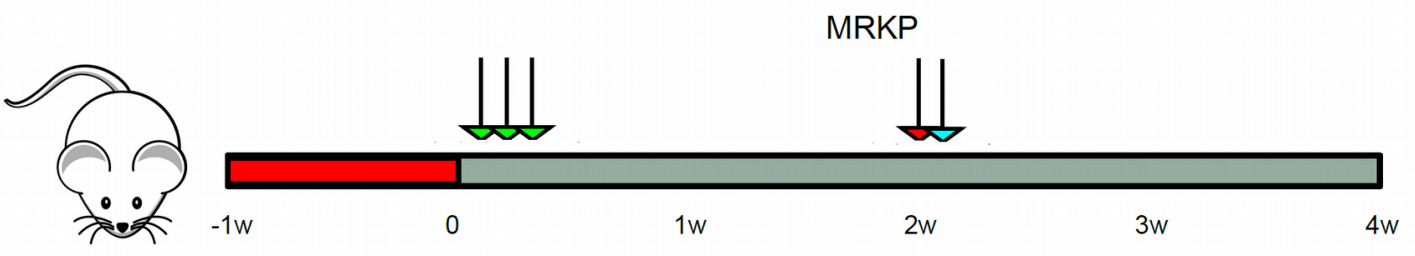

Antibiotic treatment

$\rightarrow$ Fecal sample collection and subsequent MRKP challenge

$\rightarrow$ Fecal sample collection

$\rightarrow$ Administration of fecal suspension, anaerobic culture or specific isolated commensal bacteria

Figure M1. Schematic representation of the experiments performed with the mouse model of infection with MRKP. (A) In experiments in which the capability of colonization with MRKP after different antibiotic treatments was tested, mice were infected the day before cessation of the treatment, and fecal samples were collected on following weeks in order to assess the MRKP colonization levels. (B) In experiments in which the ability of the microbiota recovery after the antibiotic withdrawal was tested, mice were challenged with MRKP 2 or 4 weeks after the end of the antibiotic treatment, and the next day a fecal sample was collected in order to assess the MRKP colonization levels. (C) In experiments in which an attempt to restore the intestinal microbiota and CR was performed, fecal suspension or bacterial culture was administered to mice during 3 consecutive days after the antibiotic withdrawal. After 11 more days (2 
weeks in total after the end of the antibiotic treatment) mice were challenged with MRKP, and on the first day post-infection a fecal sample was collected in order to assess the MRKP colonization levels.

\subsubsection{Fecal transplant and administration of specific commensal bacteria to mice}

In order to determine if $\mathrm{CR}$ could be restored after perturbation of the microbiota with antibiotic treatment, fecal content collected from untreated, healthy mice or a mix of different bacterial strains isolated from the intestine of untreated mice was administered to antibiotic-treated mice. For fecal transplant, fecal pellets from 3 untreated mice were collected and resuspended in $1 \mathrm{ml}$ of PBS just before it's administration. To each mouse $100 \mu$ l of the fecal suspension was administered by oral gavage during 3 consecutive days starting on the first day after the cessation of the antibiotic treatment (Figure M1). The isolated commensal bacterial strains were prepared in the laboratory (explained in detail later), and transported on dry ice to the animal husbandry. To each mouse $200 \mu$ l of commensal bacterial mix suspension was administered by oral gavage during 3 consecutive days starting on the first day after the cessation of the antibiotic treatment.

The administration of commensal bacterial mix for microbiota recovery was performed in 2 sets of experiments: in one of them, commensal bacterial mix was obtained by cultivation of a fecal sample from untreated mice in different cultivation media in anaerobic conditions, while in the other type of experiments, it was obtained by isolation of desired bacterial strains from fecal samples of untreated mice.

\subsection{Preparation of commensal bacterial mix obtained by cultivation of fecal samples from untreated mice in different cultivation media}

Once confirmed it was possible to restore CR and decrease MRKP levels using a fecal transplant, experiments were performed in order to identify those bacteria that are necessary for maintenance of CR. For this purpose, various microbiota anaerobic cultures were administered to mice. These cultures were obtained by cultivation of feces from untreated mice in selective media. Since the selective media provide nutrients for the growth of particular groups of bacteria present in feces, it was possible that a culture obtained from the growth of fecal samples in particular media could support the growth 
of specific bacteria necessary for CR. Later, therefore, it would be possible to grow these anaerobic cultures again in their corresponding media, and isolate those bacteria that are responsible for CR.

To obtain the desired microbiota cultures, fecal content was collected from untreated mice and resuspended in autoclave-sterilized PBS 15\% glycerol, frozen in dry ice/ethanol bath and stored at $-80^{\circ} \mathrm{C}$. Different cultivation media including, Schaedler, Gut modified media, and Sellers were prepared according to the manufacturer's instructions. Plates containing the different media were left over night in an anaerobic chamber in order to ensure anaerobic conditions for the cultivation of bacteria present in the fecal sample (most intestinal bacteria are strict anaerobs). High purity $\mathrm{H}_{2}$ is used for purging the anaerobic chamber initially and the working anaerobic gas mixture was $\mathrm{N}_{2}: \mathrm{H}_{2}: \mathrm{CO}_{2}$ proportioned at $80: 10: 10$. Fecal sample suspension was thawed and different dilutions were plated on each cultivation media in the anaerobic chamber. Plates were incubated at $37^{\circ} \mathrm{C}$ in anaerobic conditions for 1 week. Subsequently, bacteria were collected with the help of a swab from those dilutions in which individual colonies could be observed (approximately 5,000 CFUs per plate, 20 plates). Bacteria were resuspended in $2 \mathrm{ml}$ of PBS $15 \%$ glycerol $0.1 \%$ cysteine and aliquoted in 4 tubes, which were then frozen using a dry ice/ethanol bath. One tube was thaw in the anaerobic chamber and plated into the corresponding cultivation media (e.g. bacteria that was collected from Schaedler agar, was plated on Schaedler agar). The goal of this second subculture was to ensure that the bacteria that mice were going to receive by oral gavage were really capable of growing in the specific media, since subsequently we were planning to isolate individual bacteria from cultures conferring resistance against MRE. We did this second subculture because we noticed that some bacterial strains where indeed not able to grow in this second subculture although they were actually able to produce a bacterial colony the first time we grew the feces in agar media. After 1 week of growth of this second culture in the anaerobic chamber at $37^{\circ} \mathrm{C}$, bacteria were collected and resuspended as described above. Three aliquotes of commensal bacterial mix suspension were made, 1 for each day of administration to mice. 


\subsection{Preparation of commensal bacterial mix obtained by isolation of different bacterial strains isolated from fecal samples of untreated mice}

Another strategy for restoration of $\mathrm{CR}$ consisted on the administration of commensal bacterial strains, which were identified, through a series of analysis as explained in Results section, as potentially protective commensal bacteria against MRKP colonization. Isolation of these strains is explained in detail in section 3.2.1.

For the preparation of the bacterial mix, each bacterial strain was cultivated in the corresponding media in an anaerobic chamber at $37^{\circ} \mathrm{C}$. Strains belonging to Bacteroides and Lactobacillus were grown on Schaedler plates, while strains that belong to Barnesiella, Alistipes and Mucispirillum were grown on Brucella plates. As described in the previous section, all necessary material was left in the anaerobic chamber for $24 \mathrm{~h}$ prior to its use in order to ensure anaerobic conditions. Bacteria were collected from plates and resuspended in PBS 15\% glycerol $0.1 \%$ cysteine, until reaching the same absorbance (optic density-OD600 of 0.1) for all the strains. Equal volume was taken from each strain suspension in order to produce a mix of commensal bacteria that was subsequently administered to antibiotic-treated mice. As in the previous case, 3 aliquots of bacterial mix suspension were made, 1 for each day of administration.

\subsubsection{Fecal sample collection and MRE levels analysis in hospitalized patients}

In order to determine if similar bacterial species could confer protection against MRE colonization in humans and if similar mechanisms could provide $\mathrm{CR}$, fecal samples from patients with acute leukemia that were hospitalized in the Hospital La Fe (Valencia) were collected. As mentioned in the introduction, due to their immune system deficiencies, combined with low CR because of severe antibiotic therapy, these patients are often colonized with MRE, which makes them suitable for analysis. Signed permission was obtained from each patient before including them in the study. Samples were collected from December of 2013 until May of 2015. Sampling was performed weekly during all the hospitalization period that each patient underwent (patients can be hospitalized several times because they undergo several rounds of chemotherapy, subsequently some of them receive a transplant or can be admitted into the hospital due 
to associated diseases such as graft versus host disease). Thus from each patient, several samples from different time points were collected. In total, we analyzed 817 samples collected from 140 patients. Samples were kept at $4^{\circ} \mathrm{C}$ for less than $24 \mathrm{~h}$ until shipment to the laboratory. Once received, samples were aliquoted in 5 tubes and weighted. Three aliquots were resuspended in autoclave-sterilized PBS 15\% glycerol in order to preserve viability of bacteria upon freezing. One of these aliquots was used for platting the fecal sample in MRE selective media in order to confirm that the patient was colonized with MRE strain, as well as to identify the levels of intestinal colonization. No differences in the intestinal MRE levels were observed if fecal samples were directly plated or plated after being frozen at $-80^{\circ} \mathrm{C}(\mathrm{N}=30$ number of samples tested). The other 2 glycerol aliquots were kept for the isolation of commensal bacterial strains or the MRE identified in case it was required. Aliquots that were not resuspended in PBS 15\% glycerol served for the extraction of the DNA in order to determine the microbiota composition, and for the identification of metabolic and proteomic profile of each sample (tasks that are being developed by other research groups but whose results are no available yet and therefore have not been shown in the thesis). All aliquots were kept at $-80^{\circ} \mathrm{C}$ until further processing. In order to determine the effect of different factors on MRE intestinal colonization, clinically relevant data, such as immune status of the patient, antibiotics received, underlying diseases, etc. were also obtained for each patient.

\subsubsection{Experiments in rabbits for elucidating the role of dysbiosis in ERE}

In order to elucidate the association between microbiota and ERE, experiments were performed on New Zeland rabbits from a strain raised at the Polytechnic University of Valencia (89). All studies were performed in compliance with Polytechnic University guidelines. The experimental protocol was approved by the Committee of Ethics and Animal Welfare of the Polytechnic University of Valencia and followed the Spanish Royal Decree 53/2013 on protection of animals used for scientific purposes. Animals were weaned at 30 days of age and housed in separate individual cages where they had ad libitum access to food and water. The food used in the diet was standard, antibioticfree and coccidiostatic-free (Cunicebal Retirada, Nanta, Spain). In order to assess microbiota composition of rabbits, caecotrophs were collected the day of weaning, the 
day after weaning and then every other day during the next 20 days. Caecotrophs are soft feces excreted by rabbits that have a more similar chemical and microbiological composition to that of the cecal contents, where major physiological changes occur during ERE (101). Caecotroph samples were collected using neck collars to prevent their ingestion by rabbits. Samples were kept at $4{ }^{\circ} \mathrm{C}$ during approximately 3 hours and stored at $-80^{\circ} \mathrm{C}$ afterwards. Samples from mothers were also collected the day of weaning and 7 days afterwards.

A sharp decrease in food consumption is considered the first sign that can be detected in rabbits that develop ERE. For this reason, food consumption was measured daily in order to detect the onset of the disease. The day of disease onset was considered to be that one in which a rabbit diminishes food consumption $>50 \%$ compared to the previous day. The threshold of $50 \%$ was chosen since a few healthy rabbits diminished food consumption on specific days (up to $46 \%$ of reduction) without developing any other ERE signs. For ethical reasons, rabbits developing ERE were sacrificed when they ate during 2 consecutive days less than $10 \mathrm{~g}$ of food. Healthy rabbits were followed for an additional week before euthanasia (total of 3 weeks after weaning) to ensure that they did not develop any ERE compatible signs. All rabbits were sacrificed by injecting pentobarbital. To confirm ERE development, a necropsy was performed in all rabbits, and gross lesions were annotated.

\subsubsection{Food deprivation experiment}

In order to determine the effect of decreasing food consumption on ERE development and ERE associated dysbiosis, rabbits were subjected to food deprivation. Rabbits were weaned and housed in separated cages ( 1 rabbit per cage, $\mathrm{N}=28$ ). Rabbits were divided in 4 groups with 7 rabbits in each: first group was subjected to food deprivation that started on day 7 post-weaning and lasted for 2 days, the second group was subjected to food deprivation that started on day 14 post-weaning and lasted for 2 days also; third and fourth groups were control for the previous ones. In order to determine the effect of exclusively food restriction on microbiota composition changes , all rabbits that developed ERE were excluded from analysis. Food deprivation was performed on first and second week after weaning since it is when the majority of rabbits 
develop ERE. We perform food deprivation only for 2 days for ethical reasons and since we already observed major signs of the diseases and changes in the composition of the microbiota after rabbits have reduced their food consumption during 2 consecutive days. On the $8^{\text {th }}$ and $14^{\text {th }}$ day post-weaning $30 \mathrm{~g}$ of food were left in each feeder in the corresponding food deprivation group. This amount of food represents $>50 \%$ of reduction compared to the food consumption measured on the day before. The same amount was left on the following day too. Since it was impossible to obtain caecotrophs, probably because of the reduction in food intake, and in order to determine microbiota composition from rabbits in groups subjected to food deprivation, the third day after the initiation of the experiment all rabbits were sacrificed as previously described and the cecal content was collected. In addition, a necropsy was performed in order to detect any lesions compatible with ERE.

\subsubsection{Infection of healthy rabbits with the putative infectious agent isolated from ERE rabbits}

Operational Taxonomic Unit 31 (OTU- corresponds approximately to the phylogenetic level of species using a sequencing approach for taxonomic classification), classified as Clostridium, was identified as a putative infectious agent in ERE. In order to test its capacity of promoting ERE, healthy rabbits were exposed to this bacterial strain. Experiment was performed on SPF New Zealand White rabbits in the animal facility located at Principe Felipe Research Center (Valencia). Animals had ad libitum access to food and water. Food consumption and weight gain were measured daily. Clostridium strain was grown in TSA blood agar plates in anaerobic conditions at $37^{\circ} \mathrm{C}$. Bacteria were collected with a swab and resuspended in PBS $0.1 \%$ cysteine on the day of infection. Aliquots of $1.2 \mathrm{ml}$ were made for each rabbit $\left(10^{8} \mathrm{CFUs} / \mathrm{ml}\right)$. All aliquots were introduced in anaerobic atmosphere generation bags and transported on ice to the husbandry at Principe Felipe Research Center. To each rabbit $1 \mathrm{ml}$ of suspension containing the putative infectious strain classified as Clostridium was administered orally with the help of a nasogastric tube (total of 10 animals). PBS was administered to another group of 10 animals as control. A third group of 10 rabbits received $1 \mathrm{ml}$ of cecal contents from rabbits that developed ERE. This last group of rabbits was considered to be a positive 
control, since a previous study was able to reproduce the disease symptoms administering cecal contents of ERE rabbits to SPF rabbits (92).

\subsection{Isolation and characterization of intestinal bacteria from mice and rabbits}

Isolation of bacterial strains associated with ERE, as well as isolation of bacterial strains that were identified as potentially protective against MRKP colonization in mice was performed following the same protocol, which is explained in detail below.

\subsubsection{Cultivation and isolation of the intestinal bacteria}

In order to isolate intestinal bacteria that was associated with protection against MRKP colonization in mice or with ERE onset in rabbits, fecal samples from these animals were collected. Murine fecal samples were resuspended in PBS 15\% glycerol, frozen in a dry ice/ethanol bath and stored at $-80^{\circ} \mathrm{C}$ until its subsequent cultivation in anaerobic conditions. For isolation of the Clostridium strain associated with ERE onset, a fecal sample from a rabbit suffering ERE and with the highest abundance of the OTU identified as a putative infectious ERE agent, was thaw in an anaerobic chamber, resuspended in PBS and cultivated in TSA blood agar plates.

Mouse and rabbit fecal samples were cultivated in different cultivation agar media, such as BHI, Brucella, GUT, Schaedler, TSA blood agar, Columbia blood agar, etc. at various dilutions, ensuring the growth of individual colonies. Both for rabbit and murine fecal samples, bacteria were grown in anaerobic conditions at $37^{\circ} \mathrm{C}$ during 1 week. Every day, colonies that had grown enough to be visible were isolated, allowing isolation of both fast and slow growing ones. At least 5 colonies of every morphology were picked with a pipet tip and resuspended in $20 \mu$ of PBS. One part of the volume $(2 \mu l)$ of the suspension were grown again on the corresponding media plate (what was collected from TSA blood agar, was grown on TSA blood agar). The rest of the volume was kept at $4^{\circ} \mathrm{C}$ and used for further analysis, which included taxonomic identification of the colony. Taxonomic identification was performed by 16S rRNA gene sequencing. For this purpose, polymerase-chain reaction (PCR) and Sanger were performed as described below. Commensal bacterial strains of interest, as identified by 16S rRNA sequencing, were subsequently resuspended in PBS 15\% glycerol and stored at $-80 \mathrm{C}$. 


\subsubsection{S rRNA gene PCR of isolated intestinal bacteria}

In order to isolate murine commensal bacteria associated with resistance against MRKP and putative etiological agent of ERE from rabbits' fecal samples amplification of the bacterial 16S rRNA gene of each colony was performed. Briefly, for each sample, $25 \mu 1$ reaction was prepared containing $1 \mu l$ of 1 bacterial colony resuspended in PBS, $2.5 \mu 1$ 10x Standard Taq Reaction Buffer (New England BioLabs), $0.25 \mathrm{mM}$ of deoxynucleoside triphosphates (dNTPs), $2.5 \mathrm{U}$ of Taq DNA Polymerase (New England BioLabs) and $0.2 \mathrm{mM}$ of primers. The volume was completed with water. The list of primers used in the PCR is shown in Table M1. Cycling conditions were $94^{\circ} \mathrm{C}$ for 5 minutes, and 35 cycles of $94^{\circ} \mathrm{C}$ for 30 seconds, $56^{\circ} \mathrm{C}$ for 30 seconds and $68^{\circ} \mathrm{C}$ for 30 seconds, and a final elongation cycle at $68^{\circ} \mathrm{C}$ for 5 minutes. The amplification was confirmed through electrophoresis by loading $4 \mu \mathrm{l}$ of the PCR reaction on a $1.6 \%$ agarose gel. The rest of the volume was purified on ExcelaPureTM 96-Well PCR Purification Plates (Edge Bio). In order to obtain the sequence of the amplified products, a Sanger reaction was performed, as described in the next section, using as substrates the obtained amplicons.

In order to isolate the Clostridium strain associated with ERE, colonies obtained by cultivation of rabbit fecal samples were amplified using primers (Table M1) that were specific of the 16S rRNA sequence of the Clostridium OTU identified to be associated with ERE development (see Results section 4.3.4). Specific primers were designed using the Primrose program. Briefly, FASTA file with 16S rDNA sequences that are representative for every OTU detected in the fecal sample used for isolation were used to construct a database, which is used for the design of primers. OTUs sequences from the particular fecal sample were obtained through 16S rRNA high-throughput sequencing, as described in section 3.6. This approach allowed to select primers specific for the $16 \mathrm{~S}$ rRNA sequence of the desired OTU, and that will not amplify any other OTUs present in the sample used for isolation. The maximum length of the primers was set to 20 nucleotides, while no ambiguous nucleotides were accepted. The PCR conditions utilized were the same as described above. The obtained PCR products were also confirmed through electrophoresis by loading $4 \mu$ of the PCR reaction on a $1.6 \%$ agarose gel. Colonies that gave rise to an amplified product using the specific primers and whose 
PCR product was of the expected size when separated by electrophoresis on an agarose gel, were considered of interest. Since the length of the PCR product did not cover whole 16S rRNA gene, PCR reaction was repeated for colonies of interest with universal $16 \mathrm{~S}$ rRNA primers (Table M1) covering the entire 16S rRNA sequence in order to obtain further sequencing information from the particular OTU.

All PCR products obtained by amplification of the 16S rRNA gene with universal primers of colonies isolated both from rabbits and from mice were subjected to electrophoresis on a $1.6 \%$ agarose gel in order to confirm the success of the PCR reactions. PCR products were purified on ExcelaPureTM 96-Well PCR Purification Plates. Purified 16S amplicons were used as substrate for the Sanger sequencing reaction.

\subsubsection{Obtaining the 16S rRNA gene sequence of the gut isolated bacteria using the Sanger reaction approach}

For Sanger sequencing, Big Dye Terminator v.3.1 Cycle Sequencing Kit was used (Thermofisher). Briefly, the reaction mix contained 1.6 $\mu \mathrm{l}$ of buffer; $0.4 \mu \mathrm{l}$ of Big Dye Terminator; $1 \mu \mathrm{l}$ of forward 16S rRNA gene primer (Table M1) at final concentration of $0.625 \mu \mathrm{M}$ and $1 \mu \mathrm{l}$ of the purified 16S rRNA PCR product. The total volume of the reaction was $8 \mu$. The Sanger reaction was incubated at $94^{\circ} \mathrm{C}$ for 10 seconds, $50^{\circ} \mathrm{C}$ for 5 seconds and $60^{\circ} \mathrm{C}$ for 4 minutes. For each reaction 99 cycles of the 3 incubation steps were performed. Subsequently, samples were sent to the Central Service for Experimental Research at University of Valencia for determining the 16S rRNA gene sequence. Briefly, DNA fragments were separated by length using capillary electrophoresis and detection of dye fluorescence in every nucleotide position. The output data was formatted as fluorescent peak trace chromatograms.

\subsubsection{Taxonomic identification of isolated bacteria using the 16S rRNA sequence obtained with the Sanger reaction}

16S rRNA gene sequence chromatograms were processed with the program Trev. Low quality peaks at the beginning and end of the chromatograms were manually removed. The final sequence was used for taxonomic identification of the bacteria. In the 
case of bacterial strains isolated from murine fecal samples, 16S rRNA gene sequences were aligned against the Silva database by using Silva Incremental Aligner (SINA) in RDP and default parameters. In the case of bacteria isolated from rabbit fecal samples, Blast was run against a representative 16S rRNA gene sequence of the OTU31, which was associated with ERE devlopment and was identified through 16S high-throughput sequencing as described in the Results section 4.3.4 and Methods section 3.6. Only 100\% similarity and coverage was considered as a good hit.

\subsection{Detection, isolation and characterization of antibiotic resistant pathogens}

\subsubsection{Detection of the levels of colonization with MRKP in mice}

In order to determine the levels of colonization with MRKP in mice before and after the MRKP challenge, fecal samples were collected on the day of infection and 1 day post-infection. Briefly, the fecal sample was weighted, resuspended in PBS and grown on LB agar supplemented with ampicillin $(100 \mu \mathrm{g} / \mathrm{ml})$, vancomycin $(100 \mu \mathrm{g} / \mathrm{ml})$ and neomycin $(50 \mu \mathrm{g} / \mathrm{ml})$. MRKP colonies were counted and normalized by the factor of dilution and the fecal weight.

\subsubsection{Detection of MRE fecal levels in patients}

The levels of colonization with MRE in patients with acute leukemia were measured by growing fecal samples on specific cultivation agar media. A patient was considered colonized if at least 1 MRE colony was detected in the fecal sample collected from that patient. In order to detect and measure levels of colonization with MRE we plated fecal samples on ESBL Brilliance Agar (Oxoid), since the most frequent MRE isolated from acute leukemia patients in Hospital La Fe are ESBL+ Enterobacteriaceae. ESBL agar contains Cefpodoxime, in combination with additional antibacterial agents, to inhibit non-ESBL Enterobacteriaceae. Differentiation of the most prevalent ESBLproducing organisms is achieved through the inclusion of 2 chromogens. For example, $E$. coli grown on ESBL agar forms pink colonies, while $K$. pneumoniae forms green ones. Therefore, in order to quantify the level of MRE colonization, all colonies that by morphology and color corresponded to ESBL Enterobacteriaceae were counted. The 
limit of detection of ESBL Enterobacteriaceae was 1 colony in 50mg of feces. As in case of MRKP, the number of colonies was normalized by dilution and fecal weight. Besides ESBL-producing Enterobacteriaceae, in these plates could grow other bacteria that are not MRE (e.g. Pseudomonas). For this reason, 5 colonies from each MRE positive plate were subjected to taxonomic identification, as explained in detail in the following sections, in order to corroborate that they indeed belong to the Enterobacteriaceae family. In addition, the complete antibiotic resistance pattern of the 5 selected MRE colonies per plate was also obtained, as we will explain in detail in the next sections.

\subsubsection{Characterization of MRE strains from patients' fecal samples}

From each MRE positive sample, 5 colonies were picked with a pipet tip and resuspended in PBS $15 \%$ glycerol. These colonies were kept at $-80^{\circ} \mathrm{C}$ until further processing, which included their taxonomic confirmation and determination of antibiotic resistance pattern.

\subsubsection{Taxonomic confirmation of MRE strains by Maldi-TOF MS}

All bacteria isolated from MRE positive plates and kept at $-80 \mathrm{C}$ were thawed and subsequently grown over night at $37^{\circ} \mathrm{C}$ in LB agar. Subsequently, grown bacteria were picked with a sterile loop and directly spotted onto a MALDI sample plate and allowed to air dry. The matrix solution $(1 \mu \mathrm{l})$ was layered onto each bacterial spot and allowed to air dry afterwards. Subsequently, the sample plate was introduced into the Maldi-TOF MS. The laser excites bacterial molecules in the zone where colony was spotted, which produces ions that can be registered as different spectra. The obtained spectra of each analyzed isolate are compared to a spectra database, allowing the taxonomic identification of the isolate.

\subsubsection{Determination of antibiotic resistance patterns of MRE strains using the} Vitek 2 System

Besides taxonomic confirmation, the 5 colonies isolated from the MRE positive plates were subjected to determination of their susceptibility to different antibiotics. Over 
night cultures of the bacterial isolates, grown on LB agar, were collected with a sterile loop and resuspended in $3 \mathrm{ml}$ of sterile saline solution to reach the absorbance of 0.5 . The bacterial solution was incubated with commercial antimicrobial susceptibility testing (AST) cards for Enterobacteriaceae in the Vitek 2 System. The antimicrobial agents tested were amikacin, amoxicillin-clavulanic acid, ampicillin, cefepime, cefotaxime, cefoxitin, ceftazidime, cefuroxime, ciprofloxacin, gentamicin, imipenem, ertapenem, nalidixic acid, piperacillin-tazobactam, tigecycline, and trimethoprim-sulfamethoxazole (the most frequent antibiotics for which resistance has been observed in Enterobacteriaceae). Briefly, suspension of the over night culture was loaded into each card, with wells for cultivation media containing each of the antimicrobials to be tested. Cards were incubated for several hours $(2-7 \mathrm{~h}$ depending on the growth rate of the isolate), and growth of bacteria in each of the wells was assessed. The susceptibility was determined according to Clinical and Laboratory Standard Institute (CLSI) recommendations from 2012.

In order to confirm antibiotic resistance pattern identified through Vitek2 System, 1 of the 5 tested colonies from each MRE positive plate was subjected to identification of the antibiotic susceptibility pattern through Kirby-Bauer disk diffusion method. Briefly, an over night culture was resuspended in PBS until reaching absorbance of 0.5 . One part of the volume $(200 \mu 1)$ of the suspension was pipetted over MuellerHinton agar and evenly distributed with the help of glass beads. When dried, antibiotic discs were applied and pressed down to ensure complete contact with agar surface. The following antibiotic disks were used, ampicillin $(10 \mu \mathrm{g})$, piperacillin-tazobactam $(100 / 10$ $\mu \mathrm{g})$, amoxicillin/clavulanic acid $(20 / 10 \mu \mathrm{g})$, cefoxitin $(30 \mu \mathrm{g})$, ceftazidime (30 $\mu \mathrm{g})$, ceftriaxone $(30 \mu \mathrm{g})$, ertapenem $(10 \mu \mathrm{g})$, imipenem $(10 \mu \mathrm{g})$, amikacin $(30 \mu \mathrm{g})$, gentamicin $(10 \mu \mathrm{g})$, ciprofloxacin $(5 \mu \mathrm{g})$, trimethoprim-sulfamethoxazole $(1.25 / 23.75 \mu \mathrm{g})$, colistin $(10 \mu \mathrm{g})$, tobramycin $(10 \mu \mathrm{g})$, tigecycline $(15 \mu \mathrm{g})$ and cefuroxime $(30 \mu \mathrm{g})$. Plates were incubated at $37^{\circ} \mathrm{C}$. Diameter of zone of inhibitions were measured and recorded in millimeters. Depending on the diameter of inhibition, organisms were labeled as sensitive, resistant, or intermediate, according to CLSI standards from 2012. 


\subsection{DNA extraction from collected fecal samples}

DNA isolation was performed using the QIAamp ${ }^{\circledR}$ DNA Stool Mini kit in the case of rabbits' fecal samples and QIAamp ${ }^{\circledR}$ Fast DNA Stool Mini kit in the case of mice and patients' fecal samples. Extractions were performed following the protocol provided by the manufacturer. Fecal sample was weighted before the DNA extraction was performed. For more efficient extraction of DNA from hard to lyse bacteria, the initial step was modified, as previously described (102). The modification consisted on adding $500 \mu \mathrm{l}$ of glass beads, diameter $150 \mu \mathrm{m}$, to the sample, after addition of the lysis buffer, and vortexing for 5 minutes at maximum speed (Vortex-Genie ${ }^{\circledR}$ ). This step allows the mechanical disruption of the bacterial cell wall. Subsequent steps of the DNA extraction followed the QIAamp kit protocol. Finally, DNA was eluted in $50 \mu 1$ (rabbits' and mice samples) or $100 \mu \mathrm{l}$ (patients' samples) of the elution buffer. A part of the volume $(4 \mu 1)$ was separated by electrophoresis on $0.8 \%$ of agarose gel in order to check the DNA integrity. DNA concentration was determined with Qubit ${ }^{\mathrm{TM}}$ fluorometer using the manufacturer's protocol. The rest of the DNA was stored at $-20^{\circ} \mathrm{C}$ until it was used in subsequent analysis (quantitative PCR or sequencing).

\subsection{Quantitative PCR (qPCR)}

In order to determine the total bacterial load in the samples, qPCR of 16S rRNA gene was performed. For this purpose, the KAPA SYBR ® FAST qPCR Kit was used. Briefly, for each sample, $20 \mu \mathrm{l}$ PCR duplicates were prepared with each containing $2 \mu 1$ of the DNA (see previous section) used as template, $10 \mu \mathrm{l}$ of mix provided by the manufacturer, and $0.4 \mu 1$ of forward and reverse primers at the final concentration of 0.2 $\mathrm{mM}$ (Table M1). In order to complete the volume of the reaction, $7.2 \mu 1$ of water was added.

A PCR product of the 16S rRNA gene from Enterococcus faecium C68 strain was used for obtaining a standard curve. This E. faecium 16S rRNA PCR was performed as previously described in section 3.2.2. ENDMEMO program was used in order to determine the number of $16 \mathrm{~S}$ rDNA molecules in the PCR product of E. faecium C68 
based on sequence of 16S rRNA gene and concentration of the PCR product. A standard curve was obtained by making 5-fold dilutions of the PCR product.

Cycling conditions of the qPCR were $94^{\circ} \mathrm{C}$ for 5 minutes, and 45 cycles of $94^{\circ} \mathrm{C}$ for 30 seconds, $56^{\circ} \mathrm{C}$ for 30 seconds and $68^{\circ} \mathrm{C}$ for 30 seconds, and a final elongation cycle at $68^{\circ} \mathrm{C}$ for 5 minutes. By extrapolation of results with the ones obtained with standard curve, the number of $16 \mathrm{~S}$ rRNA genes was determined for each sample. The final number of $16 \mathrm{~S}$ rRNA genes per gram of fecal sample was calculated by using the following formula:

Number of $16 \mathrm{~S}$ rDNA molecules $/ 1 \mathrm{~g}$ of feces $=\mathrm{E}^{*} \mathrm{~N} / 2 * \mathrm{~F}$

where E represents the volume of the buffer used for elution of DNA after extraction (section 3.4), $\mathrm{N}$ represents number of $16 \mathrm{~S}$ rDNA molecules obtained by qPCR, 2 stands for the volume of DNA used for the qPCR reaction, and F represents the weight (in grams) of the fecal pellet from which DNA was extracted.

\subsection{High-throughput sequencing of the 16S rRNA gene}

In order to study the composition of the intestinal microbiota, high-throughput sequencing of the $16 \mathrm{~S}$ rRNA gene was performed. Samples obtained in rabbits experiments were sequenced first using the 454-Roche sequencing platform, and were resequenced later with MiSeq Illumina technology. Samples collected from mice and patients were sequenced only on the MiSeq Illumina platform. Sequencing was performed at The Foundation for the Promotion of Health and Biomedical Research of Valencia Region (FISABIO).

\subsubsection{S rDNA high-throughput sequencing using the 454 platform}

In order to amplify the $16 \mathrm{~S}$ rRNA gene for microbiota analysis using the 454 platform, $25 \mu 1$ PCR duplicates were prepared with each containing 20ng of DNA, $2.5 \mu 1$ 10x PCR buffer, $0.25 \mathrm{mM}$ of dNTPs, $0.6 \mathrm{U}$ of Taq DNA polymerase and $0.2 \mathrm{mM}$ of primers designed to amplify the V1-V3 region: a forward primer (5'CCATCTCATCCCTGCGTGTCTCCGACTCAGNNNNNNNNTCAGAGTTTGATCMT GGCTCAG-3'), composed of 454 Lib-L primer A (underline), a unique 8-base barcode 
(Ns), linker nucleotides (bold) and the universal bacterial primer 27F primer (italics); and a reverse

primer

CCTATCCCCTGTGTGCCTTGGCAGTCTCAGGCTTACCGCGGCKGCTGGCAC-3') composed of 454 Lib-L primer B (underline), linker nucleotides (bold) and the broadrange bacterial primer 515R (italics). For each sample sequenced in the same sequencing run, a primer with a different barcode was used (Table M1). Cycling conditions were $94^{\circ} \mathrm{C}$ for 5 minutes, and 22 cycles of $94^{\circ} \mathrm{C}$ for 30 seconds, $56^{\circ} \mathrm{C}$ for 30 seconds and $68^{\circ} \mathrm{C}$ for 30 seconds, and a final elongation cycle at $68^{\circ} \mathrm{C}$ for 5 minutes. PCRs were purified using QIAquick PCR purification kit (QIAGEN) according the manufacturer's protocol. Subsequently, PCRs were quantified using QubitTM fluorometer as previously described. Subsequently, the same amount of each PCR was combined in the same pool, containing between 15 and 22 samples. Each pool of samples was sequenced using a 454 GS FLX Titanium platform following Roche recommendations.

\subsubsection{MiSeq Illumina sequencing}

The V3-V4 region of the 16S rRNA gene was amplified and sequenced using the MiSeq platform from Illumina, as described in the manual for "16S Metagenomic Sequencing Library Preparation" of the MiSeq platform (Illumina).

Briefly, for each sample, a $25 \mu 1$ reaction was prepared containing $12.5 \mathrm{ng}$ of DNA, $12.5 \mu 1$ 2x KAPA HiFi Hot Start Mix, and $0.2 \mathrm{mM}$ of primers (Table M1). Water was added to complete the volume of the reaction. In case there was not enough amount of DNA, the maximum volume $(11.5 \mu 1$ of DNA) was added to the reaction, but the number of amplification cycles was increased from 25 to 30 . Cycling conditions were $95^{\circ} \mathrm{C}$ for 3 minutes, and 25 cycles ( 30 in case of the low DNA amount) of $95^{\circ} \mathrm{C}$ for 30 seconds, $55^{\circ} \mathrm{C}$ for 30 seconds and $72^{\circ} \mathrm{C}$ for 30 seconds, and a final elongation cycle at $72^{\circ} \mathrm{C}$ for 5 minutes. The amplification was confirmed through electrophoresis by loading $4 \mu 1$ of the PCR reaction on a $1.6 \%$ agarose gel. Subsequently, the PCR product was purified with the AMPure XP beads as described in the Illumina protocol.

Next, a limited-cycle PCR reaction was performed to amplify the DNA and add index sequences on both ends of the DNA, thus enabling dual-indexed sequencing of pooled libraries. Index PCR consisted of a $50 \mu 1$ reaction containing $5 \mu$ of the DNA 
obtained from the previous PCR, $25 \mu 1$ of $2 x$ KAPA HiFi Hot Start Mix, and $5 \mu 1$ of forward and reverse indexed primers. Temperature conditions were the same as for the first reaction, but the number of cycles was reduced to 8 . The obtained PCR product was purified with the AMPure XP beads following the manufacture's protocol. An equal amount of the purified DNA was taken from each sample for pooling. Each pool of samples $(\mathrm{N}=96)$ was sequenced following Illumina recommendations.

\subsection{High-throughput $16 \mathrm{~S}$ rDNA sequencing analysis}

\subsubsection{Analysis of sequences obtained using the 454 platform}

Sequences were processed using Mothur (103). All sequences were converted to standard FASTA format. Sequences shorter than 300 bp that contained homopolymers longer than $8 \mathrm{bp}$ or undetermined bases, with no exact match with the forward primer and barcode or that did not align with the appropriate $16 \mathrm{~S}$ rRNA variable region were not included in analysis. Using the 454 base quality scores, which range from 0 to 40 (0 being ambiguous base), sequences were trimmed using a sliding-window technique, such that the minimum mean quality score over a window of 50 bases never dropped below 25. Sequences were trimmed from the $3^{\prime}$ end until this criterion was met. Sequences were aligned to the 16S rRNA gene using as a template the SILVA reference alignment (104). Potential chimeric sequences were removed using Chimera Slayer program (105). To minimize the effect of pyrosequencing errors in overestimating microbial diversity (106), rare abundance sequences that differ in 1 or 2 nucleotides from a high abundant sequence were merged to the high abundant sequence using the pre.cluster option in mothur. Sequences with distance-based similarity of $97 \%$ or higher were grouped into the same OTU (sequencing approach to roughly determine the bacterial species present in 1 sample) using the average-neighbor algorithm. A number is assigned to each OTU according to the order in which it appears in analysis (e.g. OTU31 in analysis of samples obtained from rabbits' experiments). Since different number of sequences per sample could lead to a different diversity (i.e. more OTUs could be obtained in those samples with higher coverage), in order to compare the diversity of different fecal samples, all samples were rarefied to the number of sequences obtained in the sample with the lowest 
number of sequences (see Table M2 for each study). OTU-based microbial diversity was estimated by calculating the Shannon diversity index, which takes into account the number of OTUs and the relative abundance of each OTU. Each sequence was classified using the Bayesian classifier algorithm with the bootstrap cutoff of $60 \%$ (107). In most cases, classification could be assigned to the genus level. When it was not possible to classify a sequence to a certain taxonomic level, it was assigned as "Unclassified" followed by the upper taxonomic level (in case of genus it would be "unclassified" and name of the family).

\subsubsection{Analysis of 16S rRNA sequences obtained using the MiSeq Illumina sequencing platform}

Sequences obtained by Illumina were also processed using Mothur, with some differences:

a) Due to dual sequencing technology pair-end reads are obtained for each sequence. For this reason initial trimming by quality was performed on paired ends of sequences before joining them into a single read. Parameters used for trimming are listed in Table M2. In case of sequences obtained from rabbits and mice samples, trimming was performed with Mothur, while in the case of sequences obtained from patients' samples, trimming was performed with Prinseq Lite package. Criteria for all Illumina sequence processing steps are listed in Table M2.

b) Chimeric sequences were removed using Uchime algorithm (108), since it has better sensitivity compared to previously used ChimeraSlayer (note that when the analysis of 454 sequences was performed, the Uchime algorithm was still not available in Mothur). Besides, Uchime algorithm is much faster which is important taking into account the higher number of sequences obtained by Illumina.

c) Due to the higher number of sequences obtained from Illumina runs, clustering of sequences into OTUs was performed differently than in the case of 454 pyrosequencing. Sequences obtained from rabbits and mice samples were grouped with cluster.split option in Mothur. This command splits sequences at the taxonomic level of order and performs clustering within each order. In the case of sequences obtained from 
patients' samples, clustering into OTUs was performed using the VSEARCH abundancebased greedy clustering (AGC) method (109). This algorithm, published recently allows the processing of a large number of sequences, which is the case for patients' data, since the number of samples sequenced by Illumina for this study was 817 .

d) Rare abundant sequences that differ in 1 to 4 nucleotides (see Table M2) from high abundant ones, were merged to the high abundant sequence using the pre.cluster option in Mothur. The number of nucleotides allowed to differ depends on length of the sequence ( 1 nucleotide per 100 base pairs).

\subsubsection{Principal Coordinate of Analysis and Hierarchical clustering}

In order to compare the overall microbiota similarity between different fecal samples, the Yue Clayton distances between the microbiota of pairs of samples were calculated using Mothur. These distances compare the number of shared OTUs between 2 pairs of samples. Moreover, the Yue Clayton takes into account the relative abundance of each OTU. Principal Coordinates of Analysis (PCoA) was performed on the resulting distance matrices with Mothur.

\subsection{Nextera sequencing of the genome of the Clostridium strain identified as a potential promoter of ERE in rabbits}

To determine genes and functions encoded by the bacterial strain associated with ERE, genomic sequencing was performed. The strain was grown on TSA blood agar in anaerobic conditions at $37^{\circ} \mathrm{C}$. Colonies were collected and resuspended in $1 \mathrm{ml}$ of PBS. Bacterial cells were centifuged at $13,000 \mathrm{~g}$ during 20 minutes and supernatant was discarded. DNA was extracted with the QIAamp ${ }^{\circledR}$ Fast DNA Stool Mini kit as described previously in the section 3.4.

Genomic DNA was prepared for sequencing by using Nextera XT DNA Sample Preparation Kit as described in the next section. The Nextera XT DNA Sample Preparation Kit uses an engineered transposome to simultaneously fragment and tag ("tagment") input DNA (DNA extracted from fecal sample), adding unique adapter sequences in the process. A limited-cycle PCR reaction uses these adapter sequences to 
amplify the DNA and add index sequences on both ends of the DNA, thus enabling dualindexed sequencing of pooled libraries.

Briefly, $5 \mu \mathrm{l}$ of Amplicon Tagment Mix was added to $10 \mu \mathrm{l}$ of Tagment DNA Buffer. The amount of DNA added to the mix was $1 \mathrm{ng}$. The mix was incubated at $55^{\circ} \mathrm{C}$ during 5 minutes. Neutralization of the reaction was achieved by adding $5 \mu 1$ of Neutralize Tagment Buffer. For the step of amplification, $15 \mu 1$ of Nextera PCR Mix were added. The cycling conditions were $72^{\circ} \mathrm{C}$ during 3 minutes, $95^{\circ} \mathrm{C} 30$ seconds followed by 12 cycles of $95^{\circ} \mathrm{C}$ during 10 seconds, $55^{\circ} \mathrm{C} 30$ seconds and $72^{\circ} \mathrm{C} 30$ seconds. A final elongation step was performed at $72^{\circ} \mathrm{C}$ during 5 minutes. The obtained PCR product was purified with the AMPure XP Beads as described in the manufacturer's protocol. Samples normalization and pooling was performed according to the Nextera protocol. Sequencing was performed following Illumina recommendations.

\subsection{Processing of the genome of the Clostridium strain associated with ERE}

Characterization of the strain of interest included the assembly and annotation of the data obtained by genome sequencing, evolutionary placement of the strain into the Clostridiales order, identification of the species to which it belongs, and functional annotation of the identified genes. First, sequences obtained by Illumina were processed in order to eliminate bad quality sequences. Briefly, adapter sequences were removed from the raw data using Cutadapt program (110). Quality filtering was performed using UrQt (111). Only reads with a size of $75 \mathrm{bp}$ or higher were further processed, to avoid possible misclassification of short reads. Cleaned genomic data was assembled using SPAdes v. 3.7.1 (112) using the "careful" algorithm to improve the contig reconstruction. SPAdes resulted in a draft genome assembly of 160 contigs. The average basepair coverage was 27.17X. Open-reading frames (ORFs) were identified and annotated using PROKKA (113). To maximize the annotation, ORFs were translated into amino acids and queried against 3 independent databases: Pfam v.27.0 (114), EggNOG v.4.5 (115), and KEGG (116). Annotation was performed using HMMer v.3.1.2 (E value $<0.05$, minimum coverage 0.5$)(117)$.

Predicted genes were aligned using Mafft (118) against the Multiple Sequence Alignment obtained from GenBank RefSeq that contained 92 genomes classified as 
Clostridiales. Evolutionary placement algorithm was performed to locate the most probable phylogenetic location of the isolated strain. The best phylogenetic location was considered only when the Maximum Likelihood Weight was higher than 0.95 (which translates into a probability of misplacement lower than 0.05 ).

The process of bacterial diversification and the concept of bacterial species are highly dependent on the accumulation of, both, point mutations and horizontally transferred genetic elements (119). Accordingly, the definition of species has been recently determined by the average percentage of dissimilarity between the different genomic sequences. Based on this idea, the genome drafts of isolate were compared against the reference sequences of Clostridiales. Two independent implementations were performed. First, the average nucleotide identity (ANI) was calculated using an in-house script. The query genome was fragmented in non-overlapping sequences of $1,000 \mathrm{bp}$ and queried against the reference genome. The percentage of identity was calculated for each fragment. The ANI was then calculated as the mean identity of all fragments. We considered 2 genomic sequences to belong to the same species if the ANI between them was higher than 95.0. Second, MinHash dimensionality reduction algorithm allows detecting the differences between 2 genomes by analyzing the patterns of k-mer composition (120). We computed the k-mer composition for all Clostridiales genomes, including our isolate, and compared the different k-mer profiles between them using an approximation of the Jaccard dissimilarity index. Only MASH distances lower than 0.15 were included in the analysis and distances lower than 0.05 classified 2 genomes as from the same species.

For construction of the phylogenetic tree, the core-genome of the Clostridiaceae family was constructed first. To build the core-genomes we started by assigning relations of orthology between pairs of genomes. Orthologs were defined as bidirectional best hits using end-gap free global alignment (as in Touchon et al. (121)). Hits with less than $40 \%$ similarity in amino acid sequence or more than $20 \%$ difference in protein length were discarded. A total of 229 core-genes were identified for the Clostridiaceae family. Coregenes were then translated to amino acids. The Clostridiaceae family phylogeny was reconstructed with IQ-tree using a concatenate multiple protein sequence alignment of the core-genes (122). 
To identify possible toxins, the exotoxin database DBETH was used (123). Isolate-specific proteins were queried against DBETH using BlastP v.2.2.26 (Evalue $<0.01)$.

\subsection{Statistical analysis and modeling}

\subsubsection{Statistical analysis applied in the mice studies}

In order to determine differences in the microbiota composition between groups of mice treated with a mix of antibiotics and the control group (untreated mice), LEfSe method was applied (124). LEfSe determines the features (organisms, clades, operational taxonomic units, genes, or functions) that most likely explain differences between classes (e.g. untreated mice vs. treated mice) by coupling standard tests for statistical significance with additional tests encoding biological consistency and effect relevance. Specifically, it uses the non-parametric factorial Kruskal-Wallis sum-rank test to detect features with significant differential abundance with respect to the class of interest. Biological significance is subsequently investigated using a set of pairwise tests among subclasses using the Wilcoxon rank-sum test. As a last step, LEfSe uses Linear Discriminant Analysis (LDA) to estimate the effect size of each differentially abundant feature. As a result, LEfSe suggest biomarkers that explain most of the differences between the groups analyzed. Thus, this test was used to identify the commensal bacteria that most likely protect against MRKP colonization in antibiotic treated mice.

On the other hand, for identifying differences in different taxa abundances between distinct groups of mice (e.g. mice treated with individual antibiotics compared to the untreated mice), the non-parametric Wilcoxon test was applied using wilcox.test function in the 'stats' R package. Taxa and OTUs with less than 10 counts in both groups were not included in the analysis. To adjust for multiple hypothesis testing, the false discovery rate (FDR) approach by Benjamini and Hochberg was used (125).

The non-parametric Wilcoxon test or Kruskal-Wallis (when more than 2 groups were compared) was used to determine if MRKP levels were statistically significantly different $(\mathrm{P}<0.05)$ between different groups of mice that were challenged with MRKP. 


\subsubsection{Statistical analysis applied for identification of clinical factors associated with MRE colonization levels in patients with leukemia}

In order to determine which clinical factors could be associated with MRE colonization levels, samples positive to MRE from each admission of every patient were analyzed. Statistical comparisons were conducted by using non-parametric Wilcoxon test, Kruskal-Wallis or linear regression, depending on the type of clinical factor. The following clinical variables were assessed as univariate predictors of MRE levels: gender, age, neutropenia (abnormally low number of neutrophils in blood- less than 500 absolute neutrophil counts per $1 \mu 1$ of blood), admission reason (whether the patient was admitted for receiving chemotherapy or a transplant), type of leukemia (acute myeloid or acute lymphoblastic), pathogen type (Escherichia, Klebsiella, Citrobacter, Enterobacter, Morganella, Proteus or Serratia genus), antifungal treatment, antibiotic treatment, parenteral nutrition (intravenous nutrition by which normal process of digestion is bypassed), mucositis (inflammation of the oral and gastrointestinal mucosa), type of chemotherapy (intensive or non-intensive), type of transplant (allogeneic or autologous). All clinical variables could be divided in 2 groups. First group is composed of those variables that do not vary with time (gender, type of leukemia patient suffered, etc.). Variables that have temporal character are included in the second group of clinical factors (antibiotic treatment, antifungal treatment, etc.). These clinical variables are initiated at some point after patient's admission to the hospital and last for different periods of time. Due to the different nature of the clinical variables, analysis of the effect on MRE colonization levels were performed differently and are explained in more detail below.

For analysis of the effect of clinical variables such as gender, age of the patient, type of leukemia that was diagnosed, type of MRE detected, type of admission (if the sample was collected during admission during which chemotherapy or transplant were performed), all MRE positive samples (227) were pooled and grouped according to the variable to study. For example, for gender, MRE levels were grouped in those obtained from samples collected from males or females. Subsequently, the levels of MRE detected in each group of patients were statistically compared using Wilcoxon test. If there were more than 2 groups of samples, such as the case of MRE type that colonized the patient, 
Kruskal-Wallis was applied. In order to test association between the age of the patient at the moment of sampling and the MRE colonization levels, linear regression was applied.

In order to investigate the effect of temporal variables on MRE colonization levels, paired analysis were performed. For this, levels of MRE in the sample previous to the initiation of the treatment (i.e. antibiotic treatment, antifungal treatment) or complication (i.e. mucositis, neutropenia, parenteral nutrition) were compared to levels of MRE in the following sample collected after the initiation, both samples being collected during the same admission period in the hospital. Two-tailed Wilcoxon matched-pairs signed rank test was performed in order to determine the effect of each tested clinical variable on changes in MRE levels, allowing to investigate if certain treatment or condition could be associated with increase or decrease in MRE colonization levels once the patient had been colonized. One-tailed Wilcoxon matchedpairs test was performed in Figure R20A, Figure R20B, Figure R21, Figure R22 and Figure R23, since trend toward MRE rate change was observed in previous analysis, allowing us to form a hypothesis that could be tested using one-tailed test.

In order to investigate if antibiotic treatments or underlying diseases previous to the MRE colonization could promote or decrease colonization levels, the MRE levels in the first MRE positive sample from each admission were analyzed. In order to ensure that the patient got colonized in the hospital, samples included in the analysis had to be preceded with an MRE negative sample. Due to the results obtained from the previous analysis (see Results section 4.2.2.2) in which administration of antibiotics to which MRE strains were sensitive resulted in reduction of colonization levels, all samples that were susceptible to antibiotics that were given to the patient at the moment of sampling were discarded from this analysis. Only those clinical variables that had more than 5 samples per group were analyzed. Clinical variables tested by this type of analysis were neutropenia, parenteral nutrition, mucositis, antifungal and antibiotic treatment. Since it was possible that the effect of clinical variables depended on time that passed since the occurrence of the complication or start of the treatment until the moment of sampling, linear regression was performed on MRE colonization levels depending on the time that passed between sampling and the clinical variable event. 
For confirming the independent association between MRE levels and the clinical variables under study, multivariate regression analysis was performed on all variables whose effect on change of MRE colonization levels was tested. Lasso regression (126) implemented in Matlab with positive penalty term $\lambda$ was applied in order to achieve a sparse solution. This allowed selection of only few variables sufficient for the formulation of the model that explains the variability in MRE levels.

\subsubsection{Application of the Lotka-Volterra (LV) model for the identification of} commensal bacteria associated with resistance to MRE colonization in hospitalized patients

In order to determine which bacterial strains are associated with protection against intestinal colonization with MRE, Lotka-Volterra (LV) mathematical model was applied (127). As mentioned in the introduction, this model was successfully applied on microbiome data obtained from patients colonized with $C$. difficile, identifying $C$. scindens as protector commensal bacteria against infections produced by $C$. difficile. Moreover, this result was later confirmed in a mouse model of infection with $C$. diffcile (27).

LV model permits to analyze temporal community data accounting also for time-dependent external perturbations. In other words, LV is an ecological model of microbiota dynamics that considers both species interaction networks and extrinsic perturbations, such as antibiotic treatment. The modified LV equation is presented bellow.

$$
\frac{\Delta \ln x_{i}\left(t_{k}\right)}{\Delta t_{k}}=\mu_{i}+\sum_{j=1}^{L} M_{i j} x_{j}\left(t_{k}\right)+\sum_{l=1}^{P} \varepsilon_{i l} u_{l}\left(t_{k}\right) .
$$

Here, the left part of the equation represents change in abundance of species of interest (xi), in our case MRE, over time period tk. The right part of equation has 3 terms. First term ( $\mu$ i) stands for the specific growth rate of MRE. The second term explains the sum of all the other bacteria mean abundances (xj) over time period tk and 
their coefficients of interaction with MRE (Mij). In particular, Mij > 0 stands for activation and $\mathrm{Mij}<0$ for repression. The third term stands for the sum of all timedependent perturbations ul (tk) (for instance antibiotic treatment) and susceptibility to these perturbations (cil). The left part of the equation, the abundance of our bacteria of interest, is known from plating fecal samples on ESBL Brilliance plates for detection of MRE colonization levels. The mean abundances of other bacteria present in fecal samples (xj) is obtained by sequencing of $16 \mathrm{~S}$ rRNA gene. Data about the timedependent perturbations is obtained from clinical data collected from each patient. Thus, the parameters of interest, inferred from these equations, are the growth, interaction and susceptibility parameters, $\mu \mathrm{i}, \mathrm{Mij}$ and $\varepsilon \mathrm{il}$. The equation is solved by using regularized linear regression with a suitable regularizer. This approach in combination with a suitable model evaluation reduces the risk of overfitting by finding the optimal trade-off between model complexity and predictability on unseen data. For this set of data elastic net regularization was applied (126), with positive penalty term $\lambda$. A standard approach to choose $\lambda$ is to apply $\mathrm{k}$-fold cross-validation in which the data is randomly partitioned into k equally sized subsets: $k-1$ of these are used to infer the parameters $(M, \mu, E)$ for several combinations of $\lambda$. The remaining, unseen subset is used to estimate the corresponding prediction errors. This is repeated for all $\mathrm{k}$ possible partitions into $\mathrm{k}-1$ possible training sets and one test set. To reduce random fluctuations, several rounds using different random partitionings are performed. Based on the results of this procedure, $\lambda$ as the penalty parameter with the minimal averaged prediction error on unseen data is chosen. The final model is determined by applying $\lambda$ to the complete data set.

The rate of change in MRE levels between 2 consecutive samples collected from the same admission was used as response variable for the model. The rate was calculated as the difference between logarithmic values of MRE levels in the first and subsequent samples divided by the time that passed between 2 samples. Since the absence of MRE in negative samples at the beginning of the hospitalization period could be explained by resistance to colonization or by absence of exposure to MRE, the rate of MRE change was calculated only for those pairs of samples in which the first one had MRE levels. The OTUs counts or genus counts per sample were used as predictor variables for the model. Briefly, OTUs or genus abundances from each sample (fraction 
ranging from 0 to 1 ) were normalized by the total load of bacterial DNA recovered from $1 \mathrm{~g}$ of each fecal sample (inferred from 16S rRNA qPCR). The 25 most abundant genera (Bacteroides, Enterococcus, unclassified Lachnospiraceae, Parabacteroides, Streptococcus, Escherichia, unclassified Ruminococcaceae, Alistipes, unclassified Clostridiales, Lactobacillus, Staphylococcus, Sutterella, Akkermansia, unclassified Bacteroidales, Subdoligranulum, Roseburia, unclassified Firmicutes, Blautia, Paraprevotella, Oscillibacter, Coprobacillus, Klebsiella, unclassified Bacteria, Faecalibacterium) including the group "Other" containing the remaining lower abundant genera were included in analysis. This number of genera was chosen since introduction of more than 25 genera would not allow us generation of the reliable model, due to the high number of variables included into the model. For the OTU modeling, only OTUs with abundance higher than $0.1 \%$ (25 most abundant ones) were considered for analysis, including the group "Other" containing the remaining lower abundance OTUs. The particular grouping was used to reduce sparsity in the data matrix and to avoid spurious, presumably noise-driven contributions. In case of non-detection of an otherwise present OTU or genus, a value that corresponds to detection limit was assigned ( 1 count per 10,000 sequences or 0.00001 , since it is the number of sequences obtained for each sample by Illumina sequencing- see section 3.7.2). As in case of MRE levels, for the pairs of samples that were included in the analysis, geometric mean abundance for each OTU was calculated from logarithmic values of normalized OTU abundances. Besides abundance data, all clinical variables that were statistically significant in previous univariate analysis were included into the model. To each pair of samples that entered the model a number (0 or 1$)$ was assigned for each clinical variable. For example, in case of antibiotic treatment, 1 was assigned to the pair of samples for those antibiotics that were given to the patient at moment of their sampling, and 0 was assigned in case that antibiotic was not administered at sampling. In total 132 pairs of samples from a total of 221 samples were included in the model.

Subsequently, the inference was performed as described above with $\mathrm{k}=5$. Inference and prediction algorithms were implemented in MATLAB R2012b (Mathworks). 


\subsubsection{Statistical analysis applied in the study with rabbits}

In order to determine statistically significant differences in the relative abundance of different taxa and OTUs between the group of healthy rabbits and those that developed ERE, the non-parametric Wilcoxon test was applied using wilcox.test function in the 'stats' R package. Taxa and OTUs with less than 10 counts in both groups were not included in the analysis. To adjust for multiple hypothesis testing, we used the FDR approach by Benjamini and Hochberg (125) implemented in fdr.R package. Taxa with an FDR value lower than 0.1 were considered significant. Various types of comparisons were performed. First, we investigated differences between samples of healthy rabbits and those that developed ERE, obtained after ERE onset. Since the majority of samples from sick rabbits were obtained on day 11 after weaning, samples from this same day in healthy rabbits were used for comparison. Since a few samples from sick rabbits were obtained on day 13 after weaning, the same test was applied comparing with samples of healthy rabbits collected on days 11 and 13 after weaning. Subsequently, we studied if significant changes in bacterial taxa or OTUs between both groups of rabbits could be detected before ERE onset. Accordingly, both groups were compared applying the Wilcoxon test to the relative abundance of bacterial taxa or OTUs of samples obtained the day of weaning and days 1,3,5,7 and 9 after weaning. In the comparison of day 9 after weaning, samples from 3 ERE rabbits that were already sick were not included in the analysis. Besides, samples obtained the day of ERE onset were compared using the same statistical approach with samples from healthy rabbits obtained on similar days. The majority of the samples obtained the day of ERE onset ( 7 out of 8) were collected between days 7 to 9 after weaning and 1 sample was obtained at day 12 after weaning. For this reason we compare samples obtained the day of ERE onset with samples from healthy rabbits obtained on day 7 and on day 9 after weaning. We also performed the same analysis but removing the outlier sample obtained from an ERE rabbit at day 12 after weaning. For determining if the evolution of the rabbits microbiota since the day of weaning until day 9 post-weaning is different in healthy rabbits compared to those that will develop ERE, linear model regression analysis was performed using the ' $\mathrm{lm}$ ' function implemented in the 'stats' $\mathrm{R}$ package. 
Material and Methods

In order to determine statistically significant differences in the relative abundance of different taxa and OTUs between groups of rabbits with food deprivation and control groups, Wilcoxon test with adjustment for the multiple hypothesis testing was applied. 
Role of intestinal dysbiosis on gut colonization by bacterial pathogens

Table M1. List of primers used in this thesis.

\begin{tabular}{|c|c|c|}
\hline Primers & Provider & Sequence \\
\hline $27 \mathrm{~F}^{\mathrm{a}}$ & Isogen & AGAGTTTGATCMTGGCTCAG \\
\hline $803 \mathrm{R}$ & Isogen & CTACCRGGGTATCTAATCC \\
\hline Clost.Otu31F & IDT & AGGCGGACTTTTAAGTGAGAT \\
\hline Clost.Otu31R & IDT & CGTCAGTTACAGTCCAGAGAAT \\
\hline $27 \mathrm{~F}-\mathrm{qPCR}{ }^{\mathrm{c}}$ & Isogen & AGAGTTTGATCMTGGCTCAG \\
\hline 338R-qPCR & Isogen & TGCTGCCTCCCGTAGGAGT \\
\hline $27 \mathrm{~F}-1^{\mathrm{d}}$ & Isogen & $\begin{array}{l}\text { CCATCTCATCCCTGCGTGTCTCCGACTCAGA } \\
\text { ACCAACCTCAGAGTTTGATCMTGGCTCAG }\end{array}$ \\
\hline $27 \mathrm{~F}-2$ & Isogen & $\begin{array}{l}\text { CCATCTCATCCCTGCGTGTCTCCGACTCAGA } \\
\text { ACCGGAATCAGAGTTTGATCMTGGCTCAG }\end{array}$ \\
\hline $27 \mathrm{~F}-3$ & Isogen & $\begin{array}{l}\text { CCATCTCATCCCTGCGTGTCTCCGACTCAGA } \\
\text { ACCTTCCTCAGAGTTTGATCMTGGCTCAG }\end{array}$ \\
\hline $27 \mathrm{~F}-4$ & Isogen & $\begin{array}{l}\text { CCATCTCATCCCTGCGTGTCTCCGACTCAGA } \\
\text { AGCCGAATCAGAGTTTGATCMTGGCTCAG }\end{array}$ \\
\hline $27 \mathrm{~F}-5$ & Isogen & $\begin{array}{l}\text { CCATCTCATCCCTGCGTGTCTCCGACTCAGA } \\
\text { AGCGGATTCAGAGTTTGATCMTGGCTCAG }\end{array}$ \\
\hline $27 \mathrm{~F}-6$ & Isogen & $\begin{array}{l}\text { CCATCTCATCCCTGCGTGTCTCCGACTCAGA } \\
\text { AGGTTCCTCAGAGTTTGATCMTGGCTCAG }\end{array}$ \\
\hline $27 \mathrm{~F}-7$ & Isogen & $\begin{array}{l}\text { CCATCTCATCCCTGCGTGTCTCCGACTCAGA } \\
\text { ATACGCCTCAGAGTTTGATCMTGGCTCAG }\end{array}$ \\
\hline $27 \mathrm{~F}-8$ & Isogen & $\begin{array}{l}\text { CCATCTCATCCCTGCGTGTCTCCGACTCAGA } \\
\text { CACCACATCAGAGTTTGATCMTGGCTCAG }\end{array}$ \\
\hline $27 \mathrm{~F}-9$ & Isogen & $\begin{array}{l}\text { CCATCTCATCCCTGCGTGTCTCCGACTCAGA } \\
\text { CATCTCTTCAGAGTTTGATCMTGGCTCAG }\end{array}$ \\
\hline $27 \mathrm{~F}-10$ & Isogen & $\begin{array}{l}\text { CCATCTCATCCCTGCGTGTCTCCGACTCAGA } \\
\text { CAGACTCTCAGAGTTTGATCMTGGCTCAG }\end{array}$ \\
\hline $27 \mathrm{~F}-11$ & Isogen & $\begin{array}{l}\text { CCATCTCATCCCTGCGTGTCTCCGACTCAGA } \\
\text { CCAACCATCAGAGTTTGATCMTGGCTCAG }\end{array}$ \\
\hline $27 \mathrm{~F}-12$ & Isogen & $\begin{array}{l}\text { CCATCTCATCCCTGCGTGTCTCCGACTCAGA } \\
\text { CCAAGCTTCAGAGTTTGATCMTGGCTCAG }\end{array}$ \\
\hline $27 \mathrm{~F}-13$ & Isogen & $\begin{array}{l}\text { CCATCTCATCCCTGCGTGTCTCCGACTCAGA } \\
\text { CCTAGGTTCAGAGTTTGATCMTGGCTCAG }\end{array}$ \\
\hline $27 \mathrm{~F}-14$ & Isogen & $\begin{array}{l}\text { CCATCTCATCCCTGCGTGTCTCCGACTCAGA } \\
\text { CCTTCCATCAGAGTTTGATCMTGGCTCAG }\end{array}$ \\
\hline $27 \mathrm{~F}-15$ & Isogen & CCATCTCATCCCTGCGTGTCTCCGACTCAGA \\
\hline
\end{tabular}


Material and Methods

\begin{tabular}{|l|l|l|}
\hline \multicolumn{1}{|c|}{ Primers } & \multicolumn{1}{|c|}{ Provider } & \multicolumn{1}{c|}{ Sequence } \\
\hline $27 \mathrm{~F}-16$ & Isogen & $\begin{array}{l}\text { CGAAGCATCAGAGTTTGATCMTGGCTCAG } \\
\text { CTCGACATCAGAGTTTGATCMTGGCTCAG }\end{array}$ \\
\hline $27 \mathrm{~F}-17$ & Isogen & $\begin{array}{l}\text { CCATCTCATCCCTGCGTGTCTCCGACTCAGA } \\
\text { CTCGAGTTCAGAGTTTGATCMTGGCTCAG }\end{array}$ \\
\hline $27 \mathrm{~F}-18$ & Isogen & $\begin{array}{l}\text { CCATCTCATCCCTGCGTGTCTCCGACTCAGA } \\
\text { CTGACACTCAGAGTTGATCMTGGCTCAG }\end{array}$ \\
\hline $27 \mathrm{~F}-19$ & Isogen & $\begin{array}{l}\text { CCATCTCATCCCTGCGTGTCTCCGACTCAGA } \\
\text { GACGACATCAGAGTTGATCMTGGCTCAG }\end{array}$ \\
\hline $27 \mathrm{~F}-20$ & Isogen & $\begin{array}{l}\text { CCATCTCATCCCTGCGTGTCTCCGACTCAGA } \\
\text { GAGTCTCTCAGAGTTTGATCMTGGCTCAG }\end{array}$ \\
\hline $27 \mathrm{~F}-41$ & Isogen & $\begin{array}{l}\text { CCATCTCATCCCTGCGTGTCTCCGACTCAGC } \\
\text { CATCCTATCAGAGTTTGATCMTGGCTCAG }\end{array}$ \\
\hline $27 \mathrm{~F}-42$ & Isogen & $\begin{array}{l}\text { CCATCTCATCCCTGCGTGTCTCCGACTCAGC } \\
\text { CGGTATATCAGAGTTTGATCMTGGCTCAG }\end{array}$ \\
\hline $515 \mathrm{R}$ & Isogen & $\begin{array}{l}\text { CCTATCCCCTGTGTGCCTTGGCAGTCTCAGG } \\
\text { CTTACCGCGGCKGCTGGCAC }\end{array}$ \\
\hline $16 \mathrm{SIlluminaFe}$ & IDT & $\begin{array}{l}\text { TCGTCGGCAGCGTCAGATGTGTATAAGAGAC } \\
\text { AGCCTACGGGNGGCWGCAG }\end{array}$ \\
\hline $16 \mathrm{SIlluminaR}$ & IDT & $\begin{array}{l}\text { GTCTCGTGGGCTCGGAGATGTGTATAAGAGA } \\
\text { CAGGACTACHVGGGTATCTAATCC }\end{array}$ \\
\hline
\end{tabular}

a Primers $27 \mathrm{~F}$ and $803 \mathrm{R}$ are universal primers for the $16 \mathrm{~S}$ rRNA gene that were used in the PCR of colonies isolated from fecal samples.

${ }^{\mathrm{b}}$ Primers Clost.Otu31F and Clost.Otu31R are specific for the OTU31 (Clostridium OTU that is associated with ERE development).

${ }^{c}$ Primers used in quantitative PCR for determination of total bacterial load in fecal samples were 27FqPCR and 338R-qPCR.

${ }^{\mathrm{d}}$ Primers from 27F-1 to 515R were used for high-throughput sequencing of the 16S rRNA gene using the 454 platform.

e Primers for high-throughput sequencing of the 16S rRNA gene using the MiSeq platform were 16SIlluminaF and 16SIlluminaR. 
Role of intestinal dysbiosis on gut colonization by bacterial pathogens

Table M2. Parameters used in processing of the sequences obtained from Illumina.

\begin{tabular}{|c|c|c|c|}
\hline Parameters & Mice & Patients & Rabbits \\
\hline \multicolumn{4}{|c|}{ Sequence trimming parameters before joining pair end reads } \\
\hline Minimum length & 251 & 250 & 200 \\
\hline $\begin{array}{l}\text { Maximum } \\
\text { homopolymer }\end{array}$ & 8 & 8 & 8 \\
\hline $\begin{array}{l}\text { Maximum } \\
\text { ambiguous base }\end{array}$ & 0 & 0 & 0 \\
\hline $\begin{array}{l}\text { Average quality of } \\
\text { bases in sliding } \\
\text { window }\end{array}$ & 25 & 25 & 25 \\
\hline Sliding window size & 50 & 50 & 50 \\
\hline \multicolumn{4}{|c|}{ Sequence trimming parameters after joining pair end reads } \\
\hline Minimum length & 440 & 440 & 440 \\
\hline Maximum length & / & 470 & / \\
\hline $\begin{array}{l}\text { Maximum } \\
\text { homopolymer }\end{array}$ & 8 & 8 & 8 \\
\hline $\begin{array}{l}\text { Maximum } \\
\text { ambiguous base }\end{array}$ & 0 & 0 & 0 \\
\hline $\begin{array}{l}\text { Minimum } \\
\text { overlapping }\end{array}$ & 25 & 25 & 25 \\
\hline \multicolumn{4}{|l|}{ Pre.cluster parameter } \\
\hline Different bases & 4 & 4 & 4 \\
\hline \multicolumn{4}{|l|}{ Subsampling } \\
\hline $\begin{array}{l}\text { Sequences } \\
\text { subsampled from } \\
\text { each sample }\end{array}$ & 10,000 & 10,095 & 10,997 \\
\hline
\end{tabular}






\section{Results}

\subsection{Microbiota dysbiosis and MRE colonization and persistence in mice}

\subsubsection{The effect of antibiotic treatment on MRE intestinal colonization and persistence}

The ability of the healthy intestinal microbiota to suppress the colonization by exogenous bacteria, including MRE, is termed colonization resistance (CR). Antibiotic treatment disrupts the microbiota and can accidentally open the way to MRE colonization. $\mathrm{CR}$ is affected differently by antibiotics of different spectrum, suggesting that some members of the microbiota participate in CR while others are dispensable (128). In order to study the effect of the microbiota dysbiosis upon antibiotic administration on colonization and persistence of MRE, we used a mouse model and an MRE strain belonging to the species Klebsiella pneumoniae (MRKP). This strain was previously isolated in Memorial Sloan Kettering Cancer Center from the bloodstream of a hospitalized patient (85). Mice received different antibiotic treatments, as described in Methods. Briefly, animals were treated during 1 week with different antibiotics frequently used in hospitals (ampicillin, vancomycin, neomycin) and infected with MRKP the day before the cessation of the treatment. Fecal samples were collected after 1 week of treatment and before infection in order to determine the microbiota composition and levels of colonization with MRKP in these mice. Yue \& Clayton measure of microbiota dissimilarity, which takes into account the similarity in the relative abundance of the identified OTUs, and PCoA were used to compare the microbiota structure of samples obtained from mice after 1 week of treatment with different antibiotics (Figure R1). The microbiota composition in samples obtained from mice treated with antibiotics differed from that one of the untreated group (AMOVA, $\mathrm{p}<0.01$ ). Specifically, mice treated with ampicillin or vancomycin had a more different microbiota compared to the control (ampicillin $p=0.005$; vancomycin $p=0.006$ ) than those treated with neomycin $(\mathrm{p}=0.01)$. 


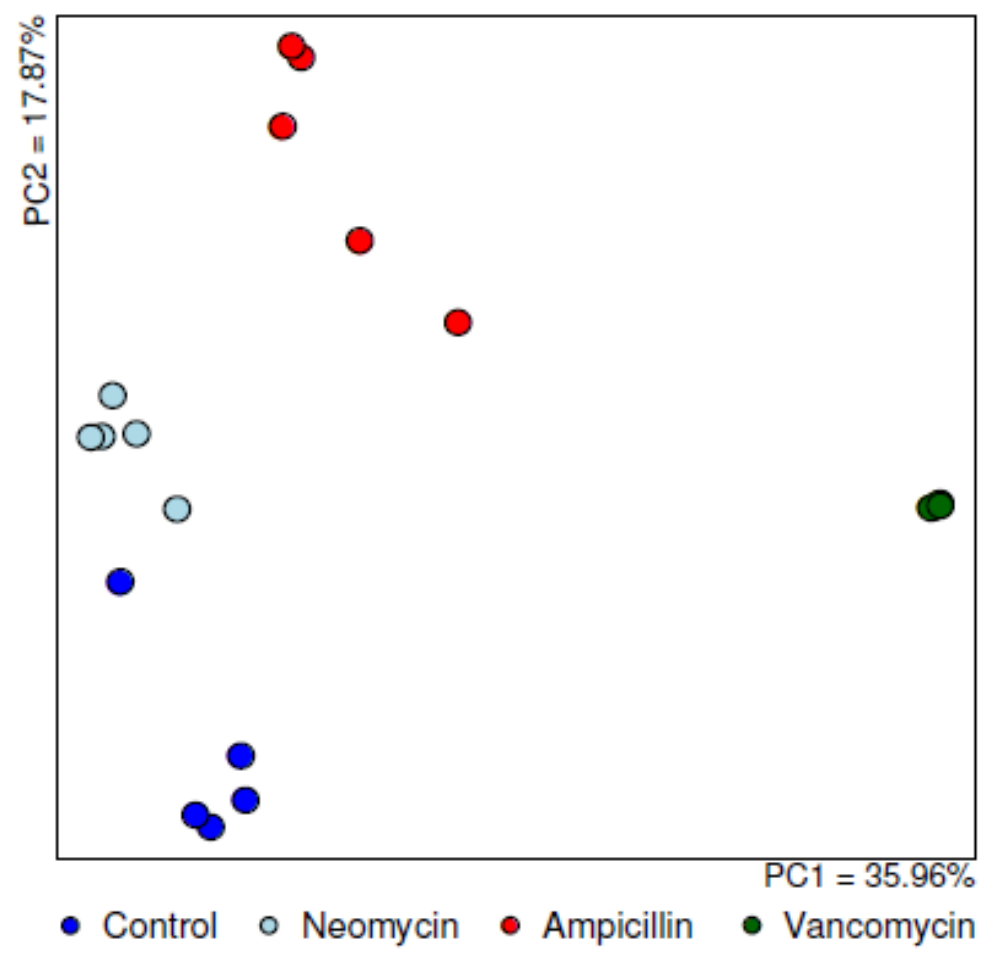

Figure R1. Effect of antibiotic treatment on murine intestinal microbiota. PCoA of the overall microbial structure obtained from Yue \& Clayton values calculated in samples collected from mice treated for 1 week with ampicillin, vancomycin and neomycin, and from samples obtained from a control group of mice that did not receive antibiotics. Each point corresponds to 1 fecal sample. Distance between 2 samples is a measure of overall microbial dissimilarity between these 2 samples- the closer they are located on the graph, more similar is their microbial composition. The 2 principal coordinates (PC1 and $\mathrm{PC} 2$ ) that explain most of the variability in the samples are shown. $\mathrm{N}=5$ mice per group.

These differences can be also observed in Figure R2, a heatmap representing the 200 most abundant OTUs identified in these mice. As can be seen in this figure, both vancomycin and ampicillin depleted most of the OTUs present in untreated mice, while neomycin treatment induced lower changes. 
Results

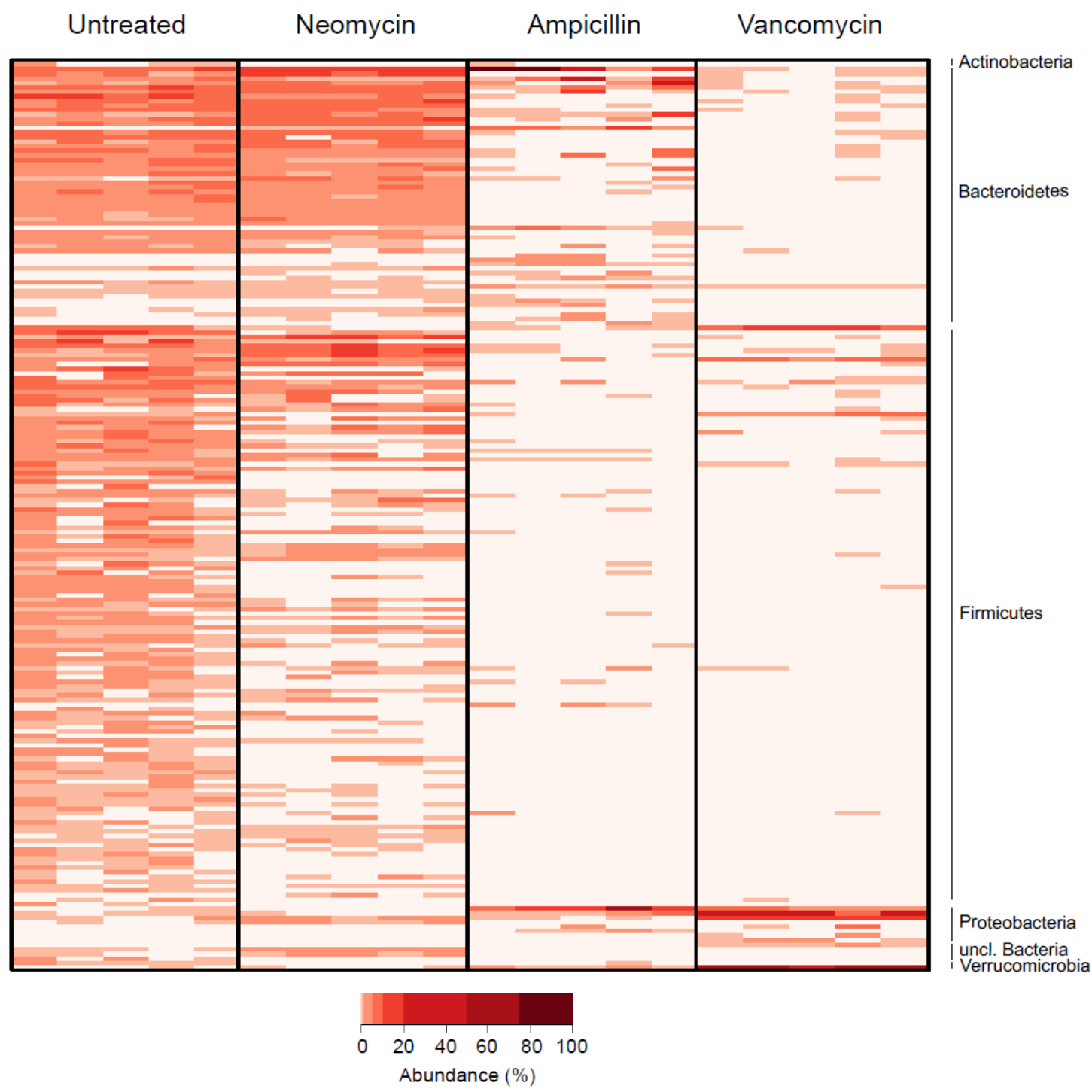

Figure R2. The effect of different antibiotic treatments on OTU abundances. While ampicillin and vancomycin treatment depleted the abundances of a high number of the OTUs present in mice, neomycin treatment induced lower changes. Heatmap representing percentage of the abundances of 200 most abundant OTUs ( $\mathrm{N}=5$ mice per group).

A similar effect of the antibiotics could be observed at the genera level (Figure R3). The abundances of 31 most abundant genera (with mean abundance $>0.01 \%$ ) did not differ after neomycin treatment (Figure R3). In contrast, mice treated with vancomycin had drastic changes at the genus level, including lower abundances of genera belonging to the phylum Bacteroidetes and Firmicutes, while genera belonging to the phylum Proteobacteria underwent a drastic expansion (i.e. Parasutterella and Escherichia). Moreover, as previously described (65), bacterial genera that represented 
minor populations in untreated mice became dominant in antibiotic-treated mice (e.g. Akkermansia expansion upon vancomycin treatment). On the other hand, mice treated with ampicillin had significantly higher levels of Proteobacteria, including the expansion of the genus Parasutterella. In order to have an estimate of the total number of changes induced by the different antibiotics, the non-parametric Wilcoxon test was applied. To avoid false positives, the FDR was subsequently applied. This analysis confirmed that ampicillin and vancomycin treatment produced drastic changes in the microbiota composition. Specifically, ampicillin treatment affected the abundances of 30 out of the 44 analyzed genera $($ FDR $<0.1)$. When using a more stringent FDR threshold $($ FDR $<0.05)$, the abundance of 29 genera differed between mice treated with ampicillin and untreated mice. Similarly, vancomycin treatment resulted in altered abundances of 39 genera $(\mathrm{FDR}<0.1)$, or 36 when a more stringent threshold was applied (FDR $<0.05)$, compared to abundances observed in untreated animals. On the other hand, neomycin treatment resulted in altered abundances of only 7 genera $(\mathrm{FDR}<0.1)$. When using a more stringent threshold $(\mathrm{FDR}<0.05)$, no differences at the genus level were found between the microbiota of neomycin treated mice compared to that of untreated animals. 
Untreated

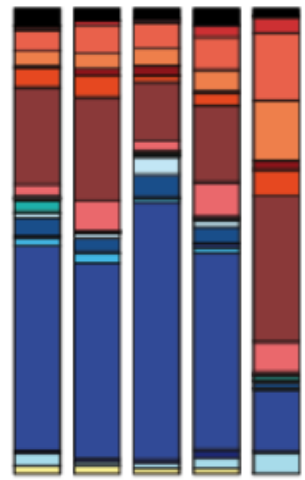

Neomycin

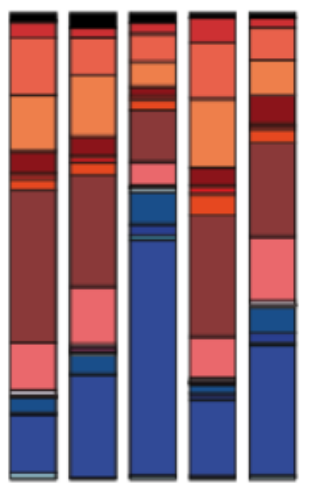

$\square$ Mucispirillum

$\square$ Enterococcus

$\square$ Lactobacillus

- Roseburia

口 unclassified_Lachnospiraceae

ㅁ Dorea

- Moryella

$\square$ Clostridium

$\square$ unclassified_Ruminococcaceae

ㅁ Oscillibacter

Ruminococcus

ㅁautia

Coprobacillus

Turicibacter

unclassified Firmicutes

u unclassified Mollicutes
Ampicillin
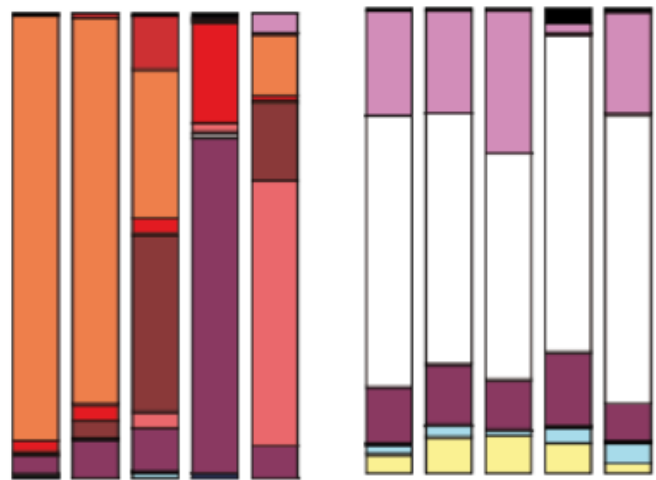

$\square$ Bifidobacterium

$\square$ Adlercreutzia

$\square$ Parasutterella

ㅁ Escherichia.Shigella

$\square$ Akkermansia

$\square$ unclassified_Bacteria

$\square$ Bacteroides

$\square$ Barnesiella

$\square$ unclassified_Porphyromonadaceae

$\square$ Parabacteroides

$\square$ Prevotella

$\square$ unclassified_Prevotellaceae

$\square$ Alistipes

$\square$ unclassified Bacteroidales

$\square$ unclassified_Flavobacteriaceae

- Other_bacteria

Figure R3. The effect of different antibiotic treatments on abundances of the 31 most abundant genera. Ampicillin and vancomycin treatment produced drastic changes in the microbiota composition (30 and 39 out of 44 analyzed genera were altered, respectively), while neomycin treatment altered abundances of only 7 genera $(\mathrm{FDR}<0.1)$. Each bar represents the microbiota of 1 mouse. The most predominant bacterial taxa (mean abundance $>0.01 \%$ ) are shown and labeled with different colors as indicated. Bacterial taxa were obtained by classification of $16 \mathrm{~S}$ rDNA sequences to the genus level using Mothur. In case a sequence could not be classified to the genus level, the closest level of classification to the genus level was given, preceded by "unclassified_". $\mathrm{N}=5$ mice per group.

Importantly, differences in the microbiota composition as a result of distinct antibiotic treatments were reflected in the levels of colonization with MRKP (Figure R4A). As expected, MRKP was not able to colonize untreated mice. On the other hand, CR was not impaired in mice treated with neomycin: MRKP was undetectable in 4 out of 5 mice treated with neomycin 2 days post-infection (Figure R4A). These results suggest that the minor changes induced by neomycin did not impair the capacity of the microbiota to confer protection against MRKP. However, ampicillin and vancomycin treatment disrupted CR and allowed MRKP to colonize the intestine at high levels. 
A

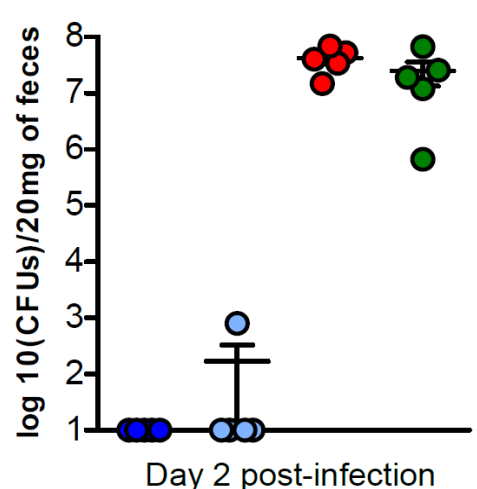

B
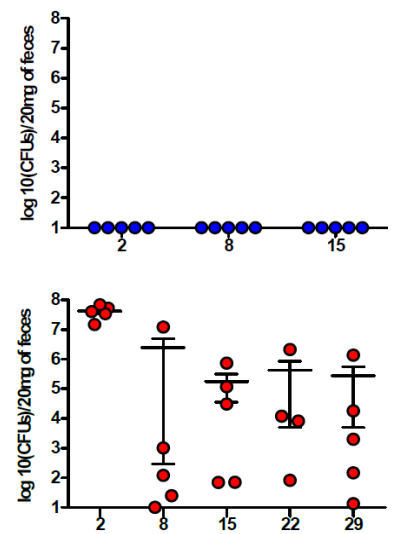
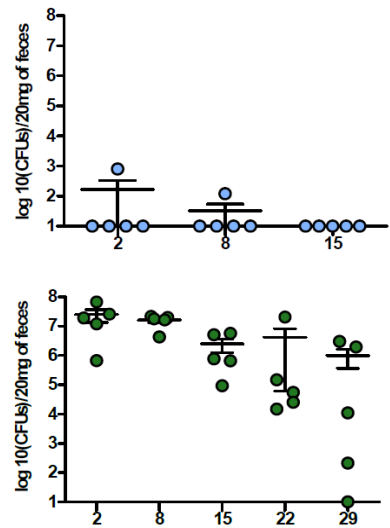

- Control o Neomycin

- Ampicillin

- Vancomycin

Figure R4. MRKP levels of intestinal colonization on days following infection in mice that did not receive antibiotics (control) or mice treated with different antibiotics. (A) MRKP intestinal levels on the day 2 post-infection: while neomycin treated mice had intact CR, mice treated with ampicillin and vancomycin were highly susceptible to MRKP colonization. (B) Mice treated with ampicillin and vancomycin remained colonized with MRKP for 1 month after the infection and antibiotic cessation, although a high variability in the intestinal levels of MRKP was observed among different mice. Values are represented in logarithmic scale and reflect the number of CFUs identified in $20 \mathrm{mg}$ of fecal material. Horizontal lines represent the mean and +/- SEM.

In order to study the capacity of the murine microbiota to recover upon antibiotic treatment and suppress MRKP in those mice in which MRKP could successfully colonize the intestinal tract (i.e. ampicillin and vancomycin treated mice), both the microbiota composition and the fecal levels of MRKP were analyzed up to 4 weeks post MRKP colonization and subsequent antibiotic withdrawal. As shown in Figure R5 (OTU level) and Figure R6 (genus level), mice treated with ampicillin or vancomycin did not completely recover their original microbiota even 4 weeks after antibiotic cessation (AMOVA, $p=0.009$ and $p=0.007$ for ampicillin and vancomycin recovery respectively compared to untreated mice). Interestingly, the level of recovery at the OTU level was different depending on the mouse. While some mice recovered the majority of the baseline most prevalent OTUs (up to 53\% of the OTUs present in all untreated mice- $\mathrm{N}=95$ ), in others, only $9 \%$ of the prevalent baseline OTUs could be detected 4 weeks after antibiotic withdrawal. 
Results

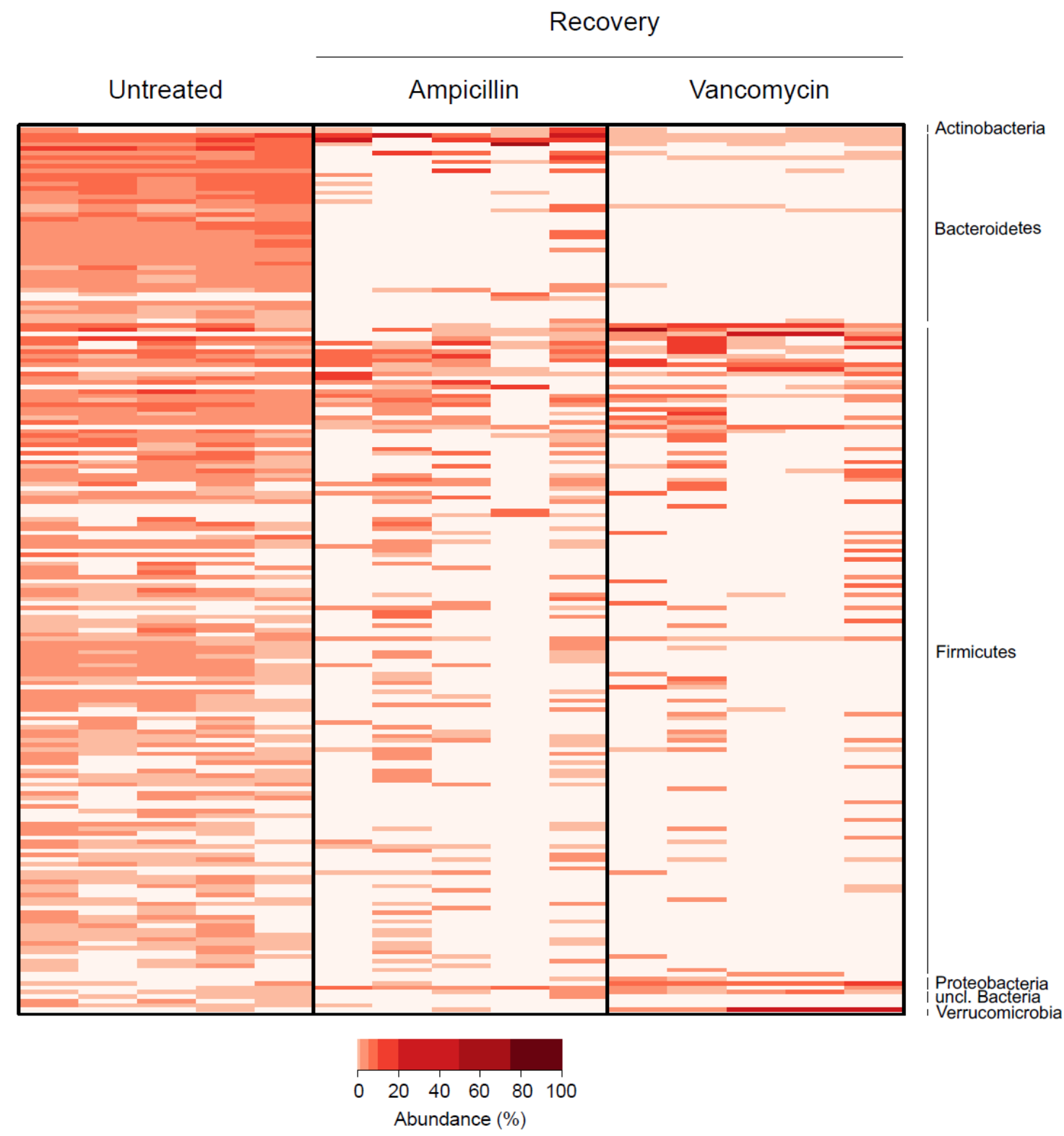

Figure R5. The capacity of the OTUs present in the mice intestine to recover upon withdrawal of antibiotic treatment. Mice treated with ampicillin or vancomycin did not completely recover their original microbiota even 4 weeks after antibiotic cessation. Heatmap representing percentage of the abundances of the 200 most abundant OTUs present in the analyzed mice ( $\mathrm{N}=5$ mice per group).

Accordingly to the incomplete recovery of the microbiota, 4 weeks postinfection, all mice treated with ampicillin or vancomycin, were still colonized with MRKP, although the levels of colonization were lower than those detected 2 days post infection (Figure R4B). Besides, a high variability in the intestinal levels of MRKP was observed among the different mice analyzed, probably due to the different microbiota 
recovery observed in these animals. These results suggest that an incomplete recovery of the microbiota upon ampicillin or vancomycin treatment may allow MRKP persistence in the intestinal tract of mice, although to a different extent depending on the mouse.

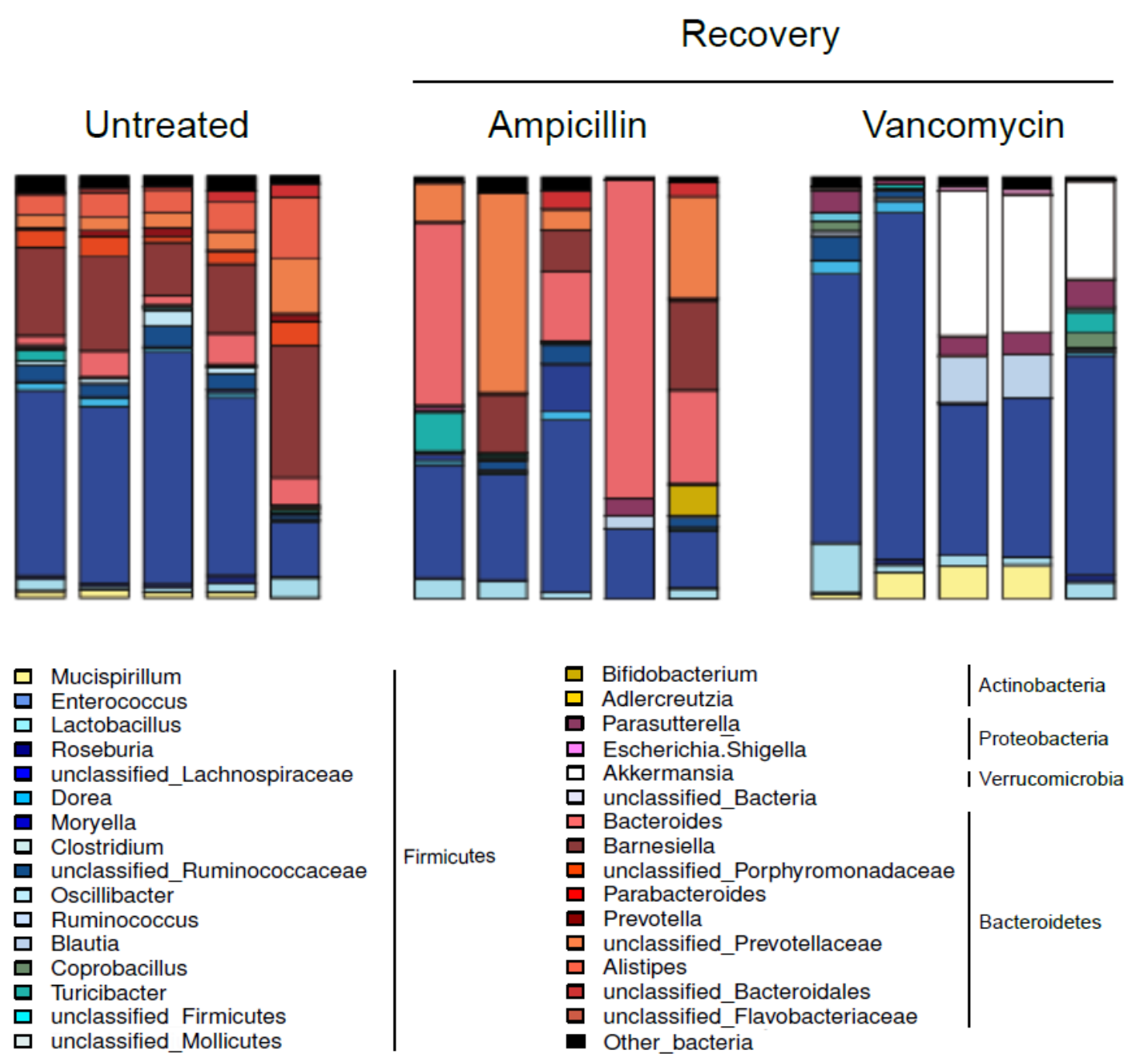

Figure R6. The capacity of the most abundant genera present in the mice intestine to recover upon antibiotic treatment. The abundances of the most abundant genera remained altered up to 4 weeks after the cessation of the ampicillin (12 genera) or vancomycin ( 29 genera) treatment (Wilcoxon test, FDR $<0.1$ ). Each bar represents the microbiota of 1 mouse. The most predominant bacterial taxa are shown and labeled with different colors as indicated. Bacterial taxa were obtained by classification of $16 \mathrm{~S}$ rDNA sequences to the genus level using Mothur. In case a sequence could not be classified to the genus level, the closest level of classification to the genus level was given, preceded by "unclassified_". $\mathrm{N}=5$ mice per group.

Although the results indicate that a single antibiotic, such as ampicillin or vancomycin, is capable of promoting MRKP intestinal colonization, hospitalized patients 
are frequently treated with multiple antibiotics at the same time. To test the effect of a combination of antibiotics on $\mathrm{CR}$, mice were treated with a cocktail of antibiotics containing ampicillin, vancomycin and neomycin (AVN). As described previously, mice were infected with MRKP 1 day before the cessation of the treatment. As expected, mice treated with AVN were highly susceptible to MRKP intestinal colonization (Figure R7). Indeed, MRKP intestinal levels 2 days post-infection were slightly higher compared to mice that received ampicillin or vancomycin alone (mean value of MRKP detected on day 2 post-infection in AVN treated mice was $1.18 \mathrm{E}+08$, while in ampicillin and vancomycin treated mice it was $4.22 \mathrm{E}+07$ and $2.48 \mathrm{E}+07$ respectively). Moreover, high MRKP intestinal levels were maintained in all mice 4 weeks upon antibiotic cessation.

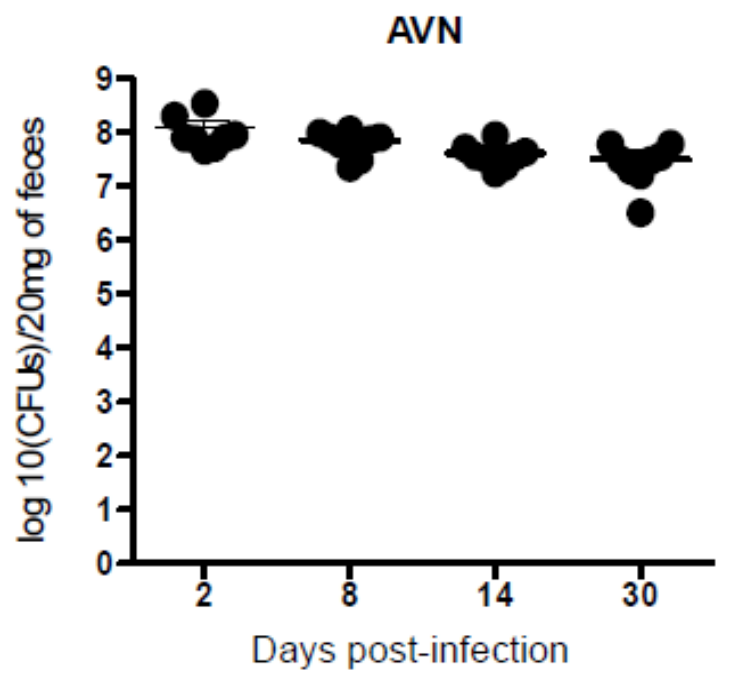

Figure R7. The effect of antibiotic cocktail on MRKP intestinal levels of colonization. MRKP fecal levels on different days, following infection of mice previously treated with $\mathrm{AVN}$ for 1 week $(\mathrm{N}=8$ mice per group). One day after infection AVN treatment was stopped. Values are represented in logarithmic scale and reflect the number of CFUs identified in $20 \mathrm{mg}$ of fecal material. Horizontal lines represent the mean and $+/$ - SEM.

Consistent with the previous obtained results, regarding the long lasting changes induced by vancomycin and ampicillin in the microbiota composition, mice treated with the combination of the 3 antibiotics and infected with MRKP did not recover their baseline microbiota even 4 weeks upon antibiotic withdrawal (Figure R8). Specifically, the abundance of 25 genera and 147 OTUs remained altered even 4 weeks after AVN cessation (Wilcoxon test, FDR $<0.1$ ). Thus, a cocktail of antibiotics promoted drastic and 
long lasting changes in the microbiota composition, and allowed MRKP persistence in the intestinal tract of mice to a higher extent than therapy with single antibiotics.

A

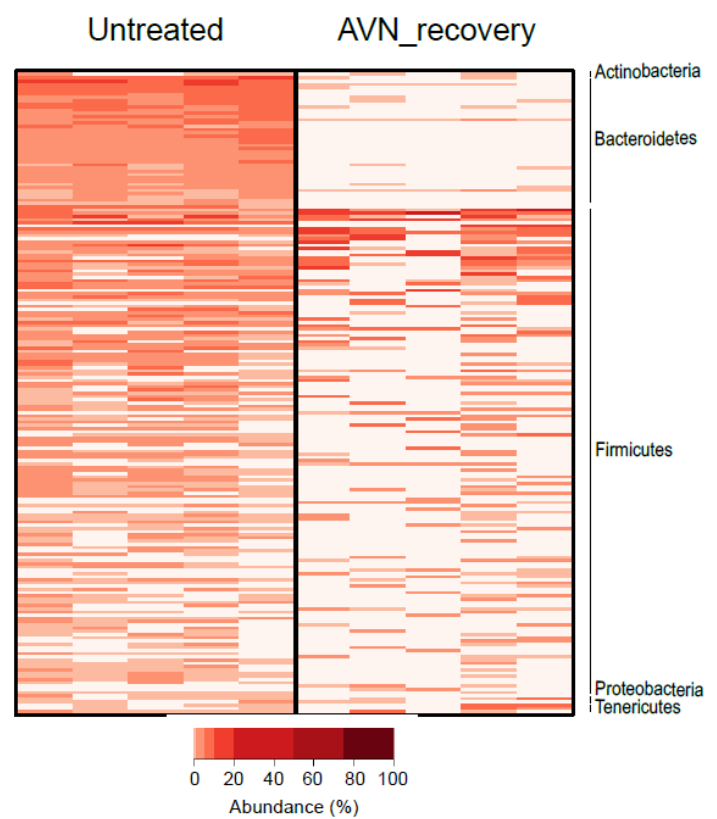

B

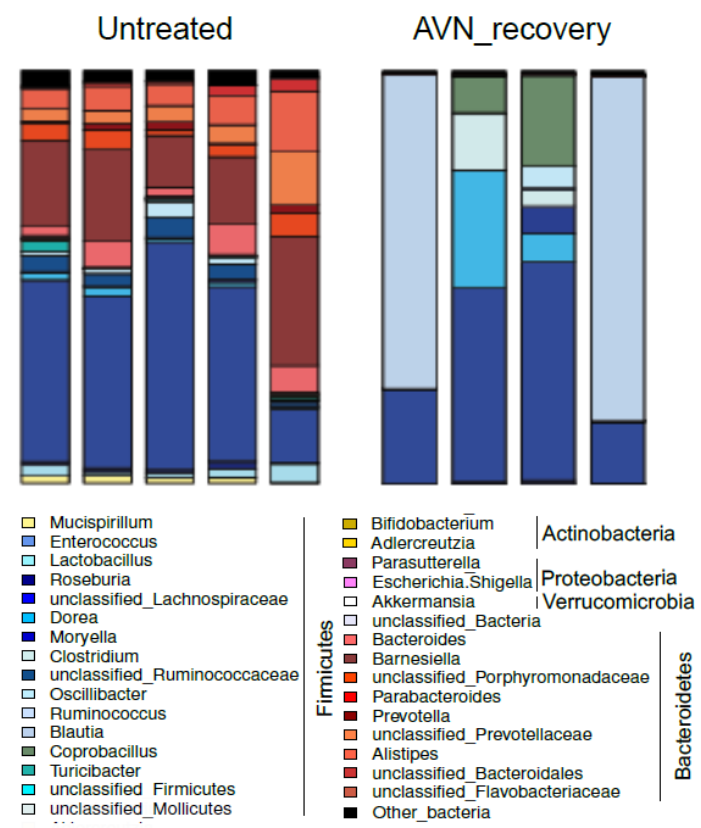

Figure R8. The long-lasting effects of an antibiotic cocktail containing ampicillin, vancomycin and neomycin on the fecal microbiota composition of mice. Mice were treated with AVN for 1 week. Subsequently mice were infected with MRKP and the antibiotic treatment was stopped. Microbiota of fecal samples was analyzed 4 weeks after antibiotic withdrawal. (A) Heatmap representing the percentage of the abundances of the 200 most abundant OTUs identified in analyzed mice. (B) Bargraphs representing the microbiota composition at the level of genus of the analyzed mice. The most predominant bacterial taxa are shown and labeled with different colors as indicated. Bacterial taxa were obtained by classification of $16 \mathrm{~S}$ rDNA sequences to the genus level using Mothur. In case a sequence could not be classified to the genus level, the closest level of classification to the genus level was given, preceded by "unclassified_". $\mathrm{N}=5$ mice per group.

\subsubsection{Long-lasting changes in the microbiota composition as a result of antibiotic treatment promote MRKP intestinal colonization}

The results obtained from previous experiments suggested that the inability of the microbiota to recover its baseline state upon antibiotic cessation allowed MRKP to persist in the mouse intestine. However, it could be possible that MRKP colonization suppresses microbiota recovery, allowing the pathogen to persist in the intestinal tract. To 
test the capacity of the microbiota to recover upon antibiotic withdrawal, in the absence of pathogen colonization, mice were treated with AVN and the microbiota was allowed to recover for 2 or 4 weeks before its analysis by 16S rRNA sequencing (Figure M1). As shown in Figure R9A, the intestinal microbiota was not able to recover even in the absence of the pathogen (AMOVA, $\mathrm{p}=0.004$ ). Importantly, mice that were allowed to recover for 2 or 4 weeks after antibiotic administration were still highly susceptible to MRKP intestinal colonization (Figure R9B). MRKP intestinal levels in both mice infected 2 or 4 weeks after the antibiotic withdrawal were significantly higher compared to those observed in untreated mice (Wilcoxon test, $\mathrm{p}<0.05$ ). Altogether, these results suggest that AVN indeed induces long-lasting changes in the composition of the microbiota, which impair $\mathrm{CR}$ and allow high levels of intestinal colonization and persistence by MRKP.

A

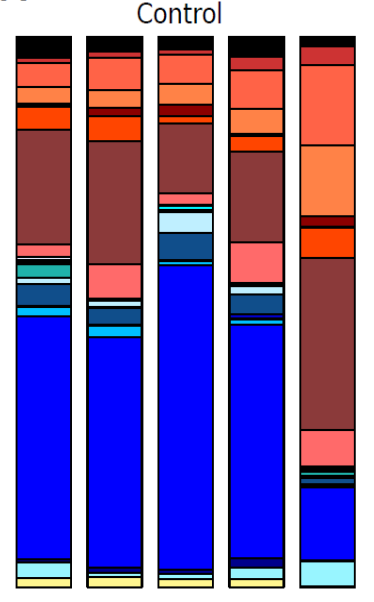

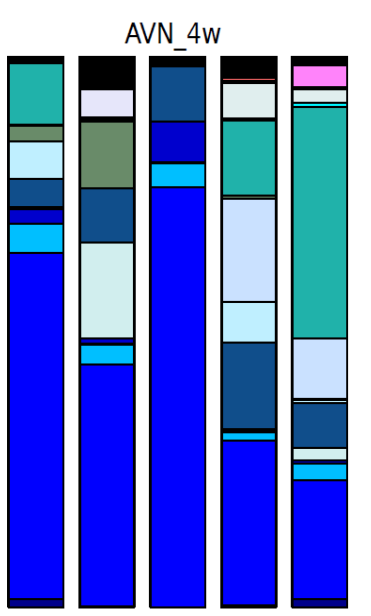

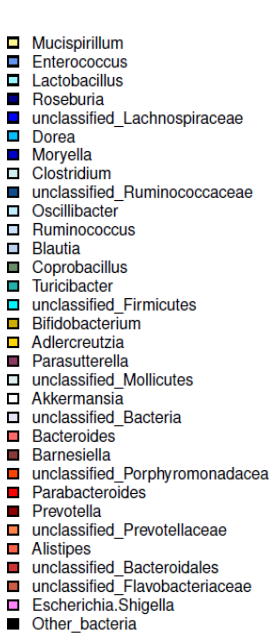

B

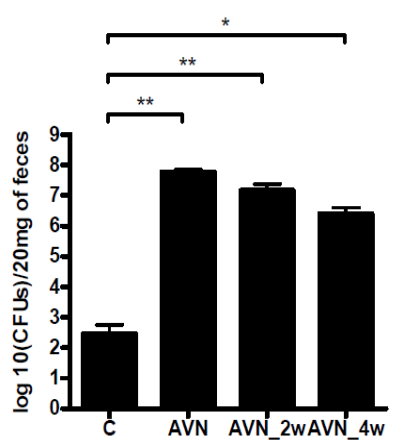

Figure R9. The long-lasting effect of antibiotic treatment on the microbiota composition and colonization resistance against MRKP. (A) Microbiota of untreated mice (Control) and mice that were treated with AVN for 1 week and left to recover for 4 weeks (AVN_4w). Each bar represents the microbiota of 1 mouse. The most predominant bacterial taxa are shown and labeled with different colors as indicated. Bacterial taxa were obtained by classification of $16 \mathrm{~S}$ rDNA sequences to the genus level using Mothur. In case a sequence could not be classified to the genus level, the closest level of classification to the genus level was given, preceded by "unclassified_". (B) MRKP levels of colonization on the first day after the infection in groups of mice that were infected (i) the day before the cessation of the AVN treatment (AVN), (ii) 2 weeks after the end of the treatment (AVN_2w), (iii) 4 weeks after the end of the treatment (AVN_4w). MRKP intestinal levels in all 3 groups were significantly higher compared to those observed in untreated mice (Wilcoxon test, ${ }^{* *} \mathrm{p}<0.01,{ }^{*} \mathrm{p}<0.05, \mathrm{~N}=5$ mice per group). Values are 
Role of intestinal dysbiosis on gut colonization by bacterial pathogens

represented in logarithmic scale and reflect the number of CFUs identified in $20 \mathrm{mg}$ of fecal material. Horizontal lines represent the mean and $+/$ - SEM. $\mathrm{N}=5$ mice per group.

\subsubsection{Restoration of the CR and suppression of MRKP colonization}

\subsubsection{Restoration of the microbiota with a fecal transplant suppresses MRKP intestinal colonization}

It has been shown previously that a fecal transplant can restore the microbiota composition upon antibiotic treatment, and suppress the intestinal colonization by pathogens such as VRE or $C$. difficile $(23,129)$. To fully demonstrate that the incomplete recovery of the microbiota upon antibiotic cessation was responsible for the intestinal colonization by MRKP, mice treated with AVN were orally gavaged with feces from healthy, untreated mice during 3 consecutive days after antibiotic withdrawal, as described in Methods (Figure M1). Mice were left for 11 days (in total 2 weeks after the end of the treatment) to re-establish the administered microbiota before the infection with MRKP was performed. Notably, microbiota composition of mice that received a fecal transplant highly resembled that one from untreated animals (Figure R10A). To test if restoration of the microbiota after antibiotic treatment restores $\mathrm{CR}$, mice were infected with MRKP and the levels of colonization were analyzed 1 day after the infection. As shown in Figure R10B, the levels of colonization 1 day after the infection were significantly lower (Man-Whitney test, $\mathrm{p}<0.01$ ) in the group of mice that received the fecal transplant compared to the group of mice that did not received it, but was also treated with antibiotics. Indeed, in 3 out of 5 mice to which fecal transplant was administered, MRKP was undetectable. Moreover, levels of colonization in mice that received the fecal transplant were not different from the levels of MRKP in mice that were not treated with AVN, and thus had an intact microbiota. These results confirm that long-lasting changes in the microbiota composition, promoted by the antibiotic treatment, are responsible for MRKP intestinal colonization. In addition, these results demonstrate that the loss of CR against MRKP upon antibiotic administration can be restored by oral gavage with feces from untreated mice. 
A

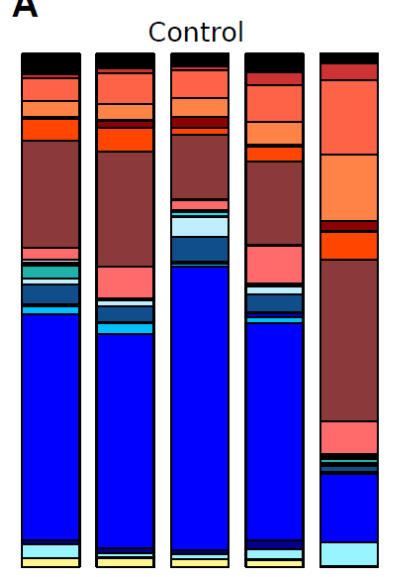

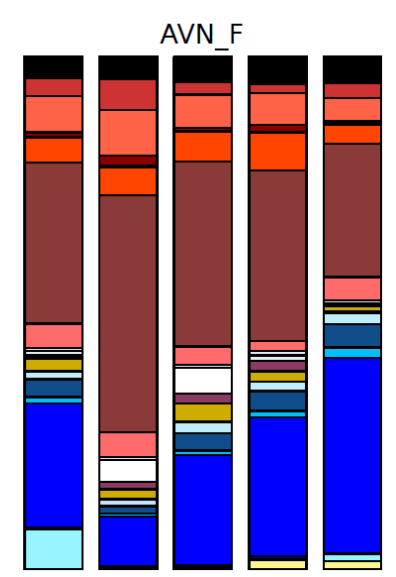

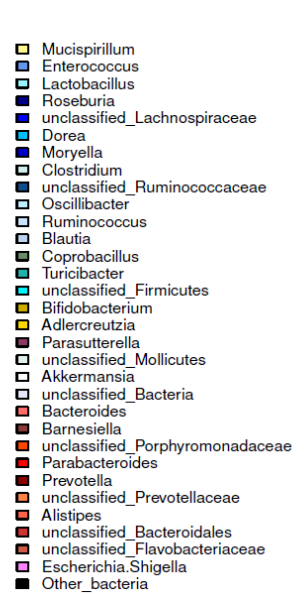

B

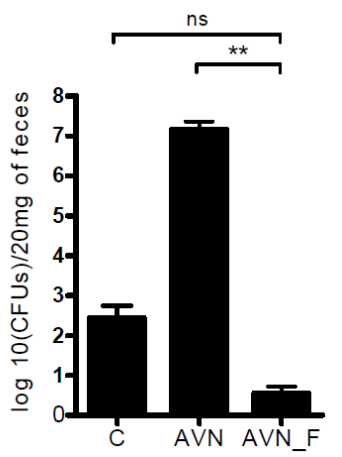

Figure R10. Restoration of the microbiota with a fecal transplant suppresses MRKP intestinal colonization. (A) Fecal microbiota, 2 weeks after AVN treatment cessation, of mice that received a fecal transplant for 3 consecutive days, starting 1 day after antibiotic withdrawal (AVN_F). For comparison, the fecal microbiota o untreated mice (Control) is shown. Each bar represents the microbiota of 1 mouse. The most predominant bacterial taxa are shown and labeled with different colors as indicated. Bacterial taxa were obtained by classification of $16 \mathrm{~S}$ rDNA sequences to the genus level using Mothur. In case a sequence could not be classified to the genus level, the closest level of classification to the genus level was given, preceded by "unclassified_". (B) MRKP levels of colonization 1 day after the infection in (i) untreated mice, (ii) mice that were treated with AVN and left to recover during 2 weeks after the end of the treatment (AVN_2W), and (iii) mice to which, besides treatment and the 2 week recovery period, fecal transplant was administered for 3 consecutive days, starting 1 day after antibiotic cessation (AVN_F). Man-Whitney test was performed for analyzing differences in colonization levels between 2 groups $\left({ }^{* *} \mathrm{p}<0.01\right.$, ns-not significant). Values are represented in logarithmic scale and reflect the number of CFUs identified in $20 \mathrm{mg}$ of fecal material. Horizontal lines represent the mean and $+/-\mathrm{SEM}$. N= 5 mice per group.

\subsubsection{Effect of the administration of fecal microbiota anaerobic cultures in restoring $C R$ against MRKP after antibiotic treatment}

Previous studies have shown that CR against VRE, lost upon antibiotic administration, could be restored not only with a fecal transplant, but also with the administration of an anaerobic culture of the fecal microbiota (23). This approach allowed the authors to identify commensal bacteria that were involved in resistance against VRE colonization, and if successful, would be of great help for the development of novel therapeutic approaches to inhibit MRKP intestinal colonization. For these reasons, an experiment was performed in order to test if CR against MRKP could be 
restored by administration of anaerobic microbiota cultures to antibiotic treated mice. For this purpose, mice treated with AVN were orally gavaged with various anaerobic cultures of the microbiota, obtained by cultivation of feces from untreated mice in different types of anaerobic media. As in the experiment explained in the previous section, mice were treated with AVN during 1 week. After the cessation of the treatment, fecal suspension or anaerobic cultures were administered during 3 consecutive days. MRKP infection was performed 2 weeks after antibiotic treatment withdrawal. Media used for cultivation of bacteria present in feces were Schaedler, Gut media, Wilkinson, and Sellers. Unfortunately, none of the cultures administered to mice treated with AVN was able to consistently suppress MRKP colonization, while fecal transplant reduced MRKP colonization levels significantly (Wilcoxon test, Figure R11).

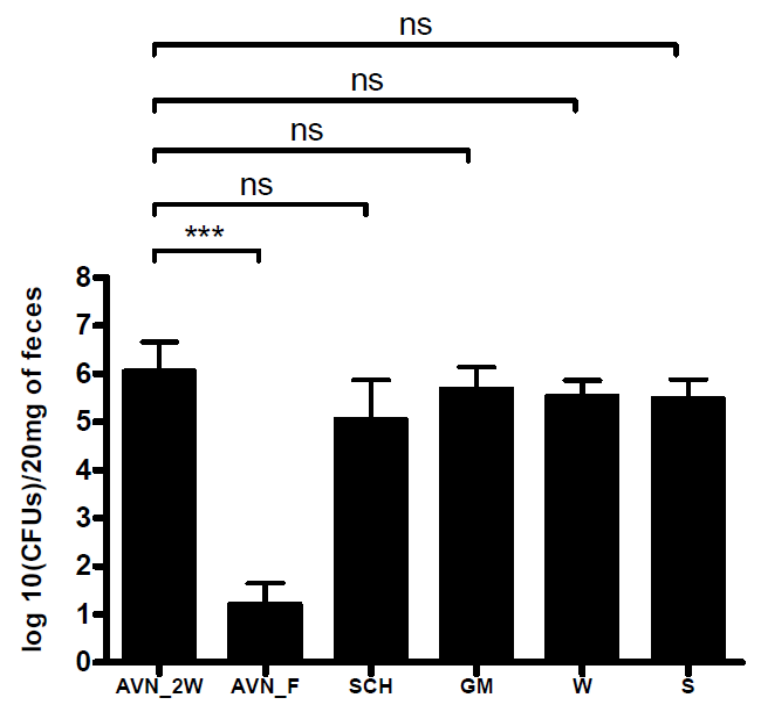

Figure R11. No anaerobic culture of the fecal microbiota suppresses consistently MRKP colonization. MRKP fecal levels 1 day after the infection of mice treated with AVN and left to recover during 2 weeks before the infection. The different groups of mice received after antibiotic withdrawal during 3 consecutive days: PBS (AVN_2W), feces from untreated mice (AVN_F) or fecal microbiota cultures obtained from the cultivation of feces in Schaedler (SCH), Gut media (GM), Wilkinson (W), and Sellers (S) cultivation media. Wilcoxon test was applied for analyzing differences in colonization levels between different groups of mice (ns-not significant, $* * * p<0.001$ ). Values are represented in logarithmic scale and reflect the number of CFUs identified in $20 \mathrm{mg}$ of fecal material. Horizontal lines represent the mean and +/- SEM. 


\subsubsection{Identification of bacteria that could provide CR against MRKP}

Since no anaerobic microbiota culture was able to suppress MRKP colonization, and in order to identify bacteria that could potentially provide CR against MRKP, an attempt to determine the commensal bacteria that were not able to recover upon antibiotic cessation was performed. For this, the microbiota of untreated mice was compared with that one of mice that received AVN and were allowed to recover for 4 weeks. Our hypotheses was that some of the commensal bacteria that were not recovered in mice susceptible to MRKP colonization could be relevant in conferring CR against MRKP. For this comparison, LEfSe algorithm for microbiota biomarker discovery was applied (124). LEfSe determines those members of microbiota (with a highest LDA score) that most likely explain differences between groups that are being compared, which is why this algorithm was used for identification of commensal bacteria that could provide protection against MRKP in antibiotic treated mice. Analysis of the microbiota composition revealed profound changes, induced by antibiotics, in mice that were treated with AVN and allowed to recover for 1 month. Specifically, LEfSe revealed altered abundances of 12 bacterial genera between the 2 groups under comparison (Figure R12A). Specifically, the genera Barnesiella, Alistipes, Bacteroides, Lactobacillus, Mucispirillum, Prevotella, Adlerecreutzia, Odoribacter, Butyricicoccus and Subdoligranulum were identified to be more abundant in untreated mice as compared to mice that recovered from AVN treatment (Figure R12A). Therefore, these bacteria represent potential candidates involved in protection against MRKP. On the other hand, the genera Staphylococcus and Dorea were represented in higher abundances in the group of treated mice. In addition, 8 more taxa were also differently abundant between these 2 groups, although it was not possible to classify them to the genus level.

After a major cultivation and taxonomic identification effort (more than 1,500 colonies were screened as described in Methods), we were able to isolate the genera with the highest LDA score (Barnesiella, Alistipes, Bacteroides, Lactobacillus and Mucispirillum, Figure R12B). As indicated in the next section, these bacteria were administered to mice treated with AVN in order to confirm their role in CR. 
A

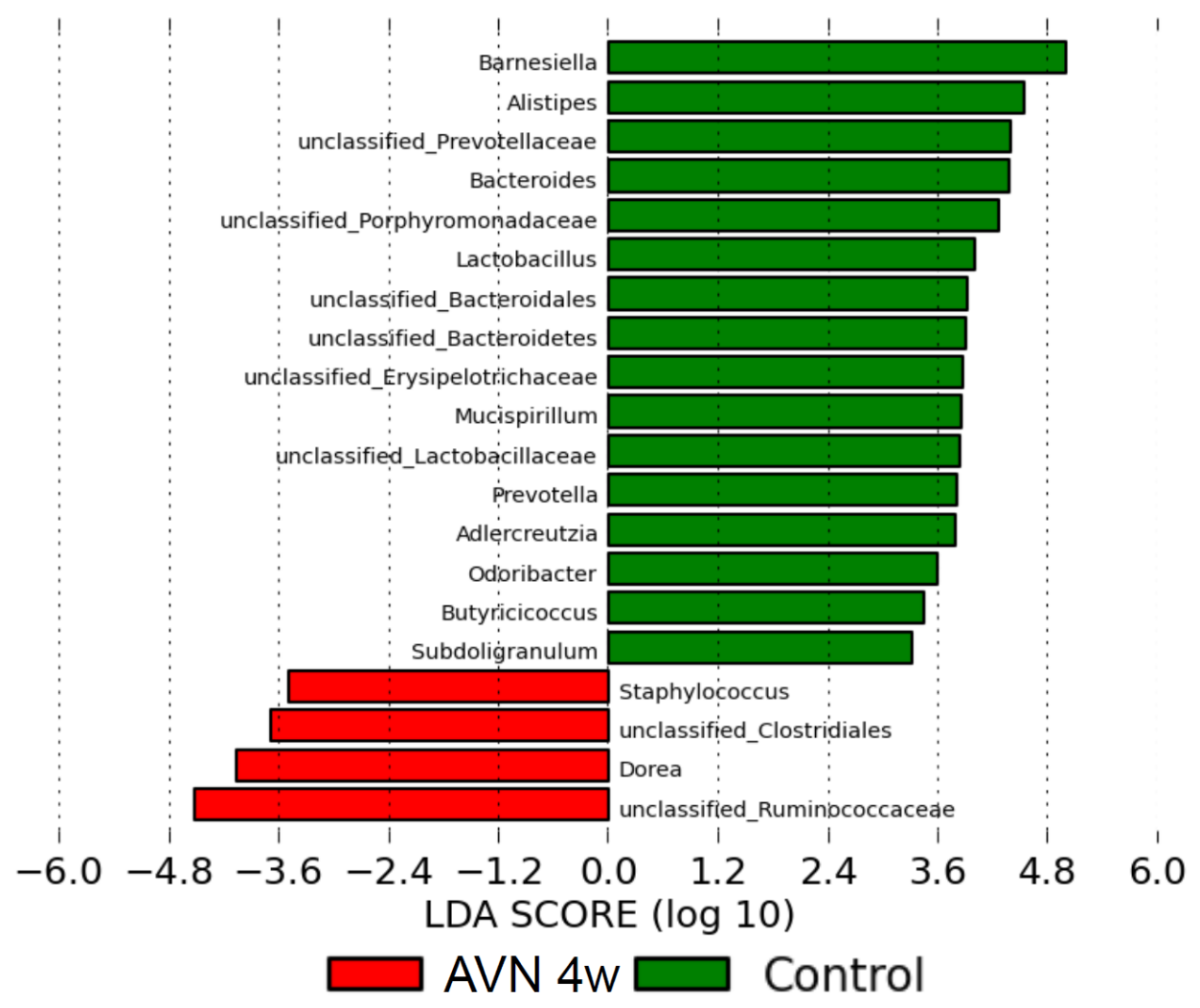

B
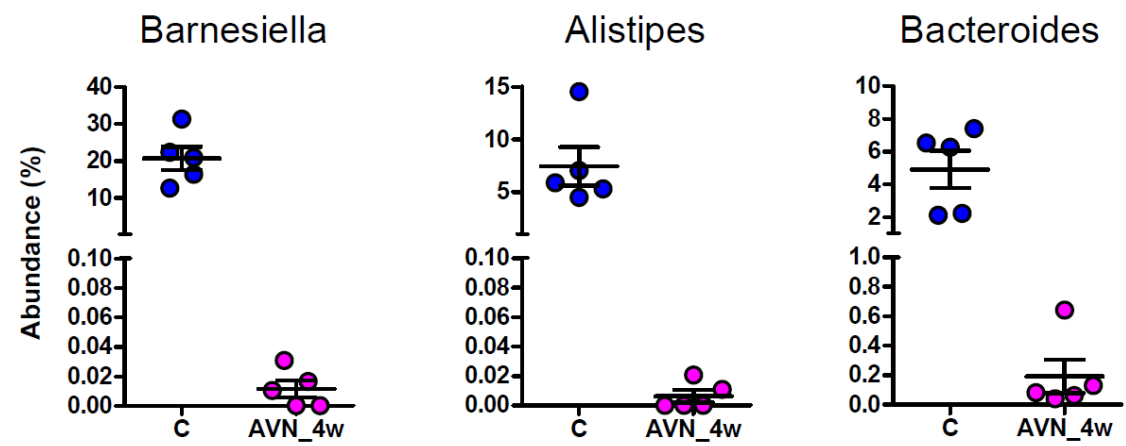

Lactobacillus
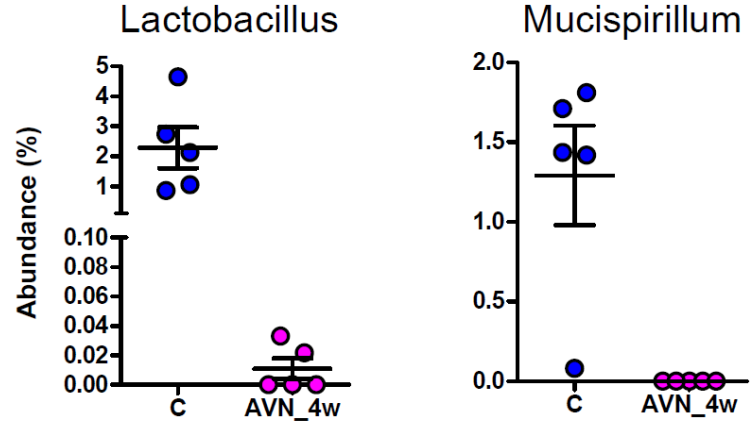

Figure R12. Changes in the microbiota composition in mice that were allowed to recover for 1 month after AVN treatment. (A) Taxa selected by LEfSe as biomarkers of dysbiosis produced by AVN treatment. In green are indicated those bacteria that were more abundant in untreated mice and in red are indicated 
those bacteria that were more abundant in AVN treated mice. (B) Relative abundances (\%) of 5 genera with the highest LDA score (those that better differentiate the microbiota of both groups of mice) were higher in untreated mice. These bacteria were selected for testing their effect on restoration of MRKP colonization resistance.

\subsubsection{A commensal bacterial mix partially restores MRKP colonization resistance after antibiotic therapy}

In order to test the role of the 5 selected bacteria that were most affected by AVN treatment on MRKP colonization resistance, a mix containing 5 murine commensal bacterial strains, each belonging to one of the selected genera (Barnesiella, Alistipes, Bacteroides, Lactobacillus and Mucispirillum) was prepared as described in Methods. The attempt to restore the intestinal microbiota was performed as in the previous experiment, in which the capacity of a fecal transplant for restoring CR was tested. Briefly, mice were treated with AVN during 1 week. Upon the cessation of the treatment, a mix containing the 5 commensals was administered to mice during 3 consecutive days. Mice were infected 11 days after the last day of bacterial administration in order to allow the establishment of the introduced bacterial strains. Importantly, administration of the mix containing Barnesiella, Alistipes, Bacteroides, Lactobacillus and Mucispirillum strains resulted in reduction of MRKP colonization levels of almost 4 orders of magnitude as compared to mice that did not received the bacterial mix (Figure R13A). Although suppression of the colonization was not complete, reduction in MRKP levels on first day post-infection was statistically significant, indicating that the bacteria present in the mix of 5 commensal strains could partially restore resistance against MRKP colonization.

Although unlikely, it was possible that the same reduction in MRKP colonization levels could be achieved with other bacterial strains. In order to confirm that the effect on $\mathrm{CR}$ produced by administration of the mix of the 5 commensal strains (MIX5) was due to the bacteria present in the mix, and would not be produced by administering any type of bacteria, anaerobic culture obtained by cultivation of feces from untreated mice in Gut media (GM) was administered to another group of mice treated with AVN. Culture harvested from Gut media was chosen since in previous experiments it was shown that administration of this anaerobic culture did not affect 
MRKP colonization levels. As in the previous experiment, mice were administered GM during 3 consecutive days, starting 1 day after antibiotic cessation. After 11 days of recovery, mice were infected with MRKP. As expected, no protection against MRKP was observed in those mice that received GM (Figure R13B).

It is important to indicate that antibiotic treatment can result in reduction of total bacterial density, which could promote MRKP intestinal colonization. In order to investigate if the effect of the MIX5 on CR was the result of restoration of bacterial density, 16S rRNA quantitative PCR was performed. Notably, the intestinal bacterial density of mice treated with AVN was lower in those mice that were allowed to recover for 2 weeks without receiving the MIX5 (Wilcoxon test $\mathrm{p}<0.01$; Figure R13C). Nevertheless, the bacterial load of fecal samples collected from mice to which GM was administered did not differ from that one of mice that received the MIX5. These results suggest that the protection conferred by the mix containing Barnesiella, Alistipes, Bacteroides, Lactobacillus and Mucispirillum strains is not the result of the recovery of the bacterial density, but rather is due to a specific mechanism that should be investigated.

A

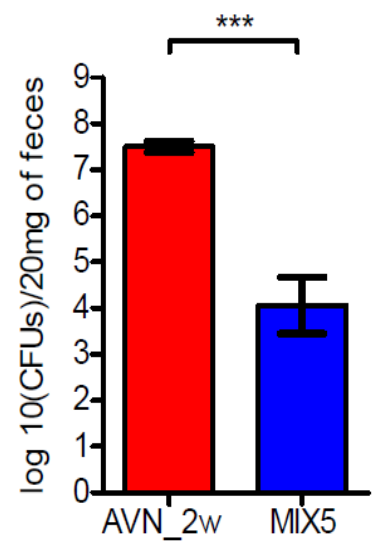

B

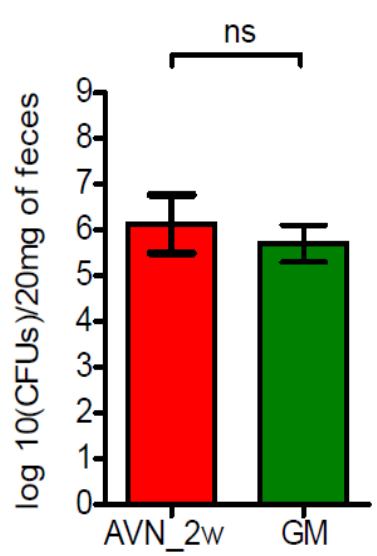

C

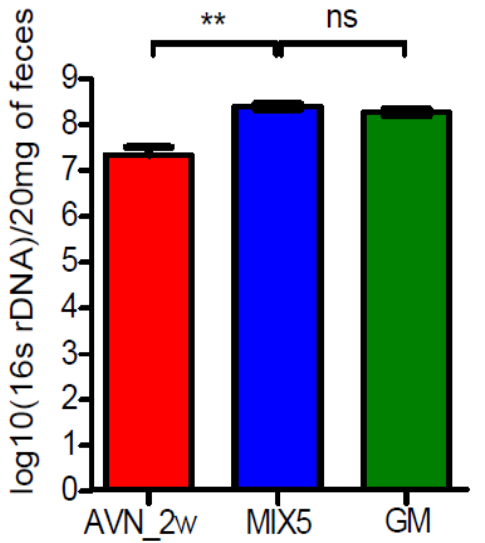

Figure R13. A commensal bacterial mix partially restores MRKP colonization resistance after antibiotic therapy. (A) Group of mice that received the bacterial MIX5 containing Barnesiella, Alistipes, Bacteroides, Lactobacillus and Mucispirillum had significantly lower levels of MRKP 1 day after the infection (Man-Whitney test, $* * * \mathrm{p}=0.0002, \mathrm{~N}=8$ mice per group from 2 independent experiments) compared to mice treated with AVN and allowed to recover without receiving the MIX5. (B) The MRKP colonization levels 1 day post-infection in mice that received anaerobic fecal microbiota culture harvested from Gut media (GM) did not differ from those identified in mice treated with AVN that were allowed to 
recover without receiving GM (N=8 mice per group from 2 independent experiments). (C) Bacterial load in samples collected from mice that received the 2 types of commensal bacterial mix quantified by qPCR of the 16S rRNA gene.

\subsubsection{Partial microbiota recovery after administration of the bacterial mix}

Sequencing was performed in order to investigate changes in microbiota produced by administration of the 5 probiotics. Yue \& Clayton measure of dissimilarity was applied in order to assess the overall microbial composition. Analysis revealed that samples obtained from mice to which Barnesiella, Alistipes, Bacteroides, Lactobacillus and Mucispirillum were administered differed from mice to which no bacterial mix was given (AMOVA p $<0.001$, Figure R14A). The same result was obtained when comparing samples obtained from mice to which GM was administered with the group that did not received any bacterial mix (AMOVA $\mathrm{p}=0.002$, Figure R14A). As expected, microbiota from mice that received the GM significantly differed from mice that received the MIX5 (AMOVA, $\mathrm{p}<0.001$ ), suggesting recovery of a distinct microbiota. Previous studies, using a mix of commensal bacteria to confer protection against intestinal colonization by other pathogens (i.e. C. difficile) have shown that a bacterial mix could restore overall microbial diversity which in turn may enhance the elimination of the pathogen (100). In order to investigate if lower levels of MRKP in the group of mice treated with the mix of 5 commensal strains could be due to an increase in overall microbial diversity, Shannon index was calculated. The obtained results indicate that there were no difference in the Shannon diversity index between mice that did not receive any bacterial mix, mice to which the mix of 5 bacterial strains was administered, and mice to which the anaerobic culture harvested from Gut media was given (One-way ANOVA, Figure R14B). Subsequently, analysis was performed in order to investigate if all 5 bacterial strains from MIX5 could re-colonize the intestine of antibiotic treated mice, and therefore, reverse the microbiota alterations produced as a result of antibiotic treatment. Notably, only Lactobacillus $(\mathrm{p}=0.0045)$ and Barnesiella $(\mathrm{p}=0.0003)$ genera were significantly more abundant in mice after the administration of the MIX5 compared with mice to which no commensal bacteria was given. Moreover, the abundances of these 2 genera in mice to which MIX5 was administered were even higher as compared with untreated mice (Wilcoxon test, Lactobacillus $\mathrm{p}=0.0016$, Barnesiella $\mathrm{p}=0.0062$ ), suggesting that 
Lactobacillus and Barnesiella not only were able to colonize the intestine of mice after antibiotic treatment, but also reach even higher levels than those observed in untreated mice. On the other hand, no increase was observed in the levels of Alistipes, Bacteroides or Mucispirillum, the other commensal bacteria administered in the MIX5. Importantly, the abundance of Lactobacillus in mice to which MIX5 was administered was similar to the levels obtained after administration of GM. Indeed, Barnesiella was the only bacterial genus included in the MIX5 that was recovered specifically in mice that received the protective mix, and was absent from mice treated with the non-protective GM, suggesting that this bacterium may be key in conferring colonization resistance against MRKP colonization in mice. 
A

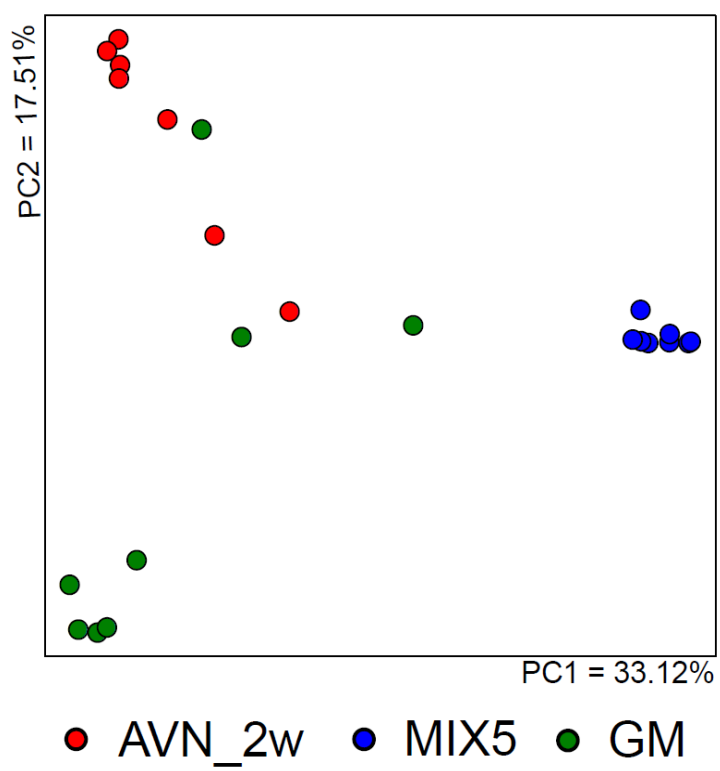

C

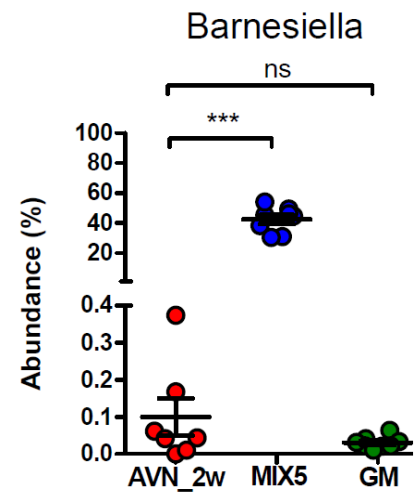

B
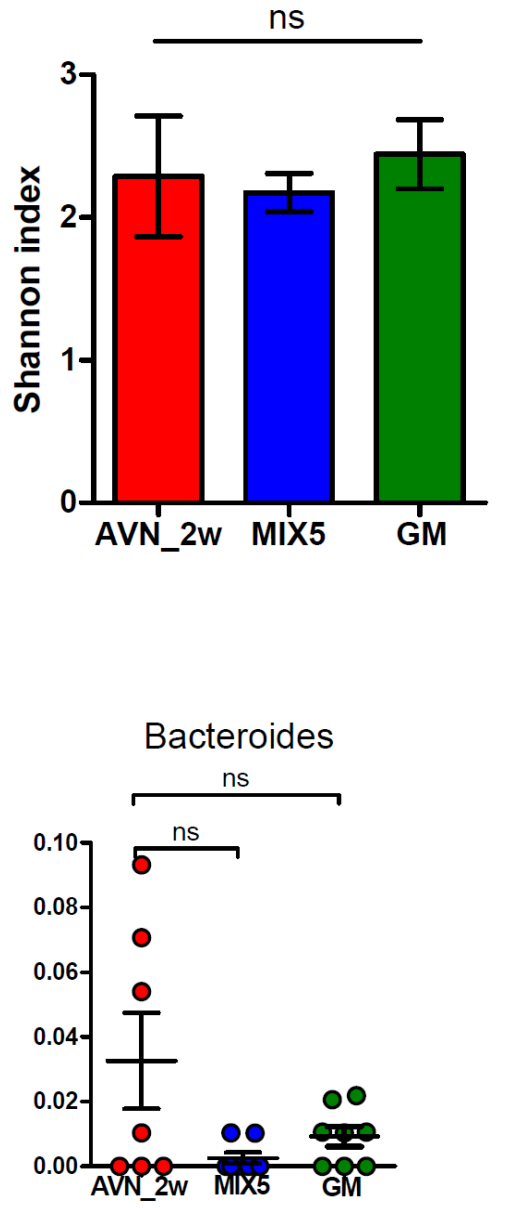

Lactobacillus

Mucispirillum
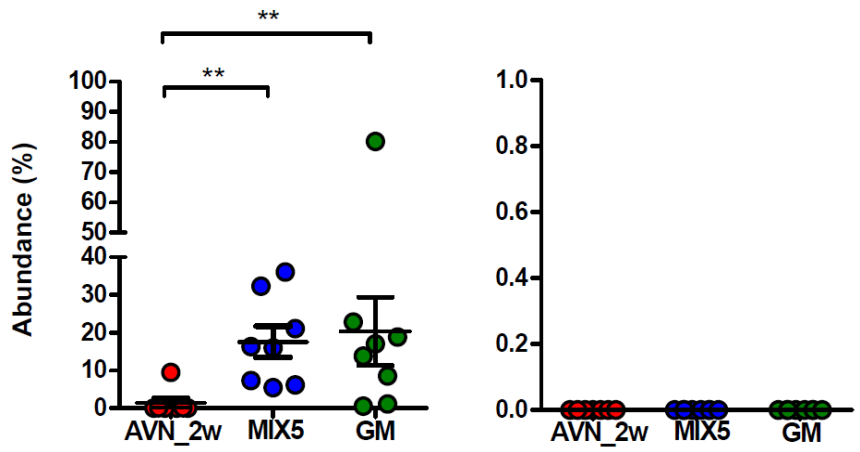

Figure R14. Partial microbiota recovery after the administration of the protective bacterial mix. (A) The overall microbial composition based on Yue \& Clayton measure of dissimilarity. Samples collected from mice to which no probiotic was given (AVN_2w) differed from samples collected from animals to which mix of 5 probiotics was administered (MIX5) and mice to which the anaerobic culture harvested 
from Gut media (GM) was administered (AMOVA, $\mathrm{p}<0.001)$. (B) Shannon index revealed similar diversity between the 3 groups of mice (One-way ANOVA, ns-not significant). (C) Abundances of the 5 bacteria that were administered in the protective mix (MIX5) in mice after administration of the MIX5, mice to which no commensal bacteria was administered (AVN_2w), and mice that received GM. Only Lactobacillus $(* * \mathrm{p}=0.0045)$ and Barnesiella $(* * * \mathrm{p}=0.0003)$ increased after administration of the MIX5 as compared to the group of mice that did not receive the mix.

Due to a deadline for writing and finishing this thesis, we were not able to perform several experiments to corroborate the role of Barnesiella in resistance against MRKP and identification of the possible mechanisms of protection. These future experiments are discussed in the Discussion section. We did test if Barnesiella or Lactobacillus could inhibit the growth of MRKP in vitro, however no inhibition could be detected suggesting that the protection against colonization is not due to the direct production of an inhibitory molecule (e.g. bacteriocins), or that this product is not synthesized in sufficient amounts under the tested conditions. 


\subsection{Microbiota dysbiosis and MRE colonization in hospitalized patients}

\subsubsection{Prevalence and characterization of MRE in acute leukemia patients}

In order to investigate how microbiota promotes $\mathrm{CR}$ and prevents colonization with MRE in humans, and if the same bacteria could be responsible for the inhibition of MRE colonization in humans as observed in mice, fecal samples from patients with acute leukemia were collected during a period of 18 months. As previously mentioned, these patients are often colonized with MRE probably due to low CR because of severe antibiotic therapy, which makes them suitable for this study. Sampling was performed weekly during their hospitalization, allowing the collection of samples from the same patient at different time points. All fecal samples were cultivated in ESBL selective media in order to detect the levels of MRE intestinal colonization. In order to confirm that strains detected by cultivation of samples in ESBL plates correspond to MRE, 5 colonies (or the number of colonies available, in case the total number of colonies per sample was $<5$ ) were isolated from each positive plate and subjected to determination of sensitivity to different antibiotics and taxonomic identification. All strains isolated from acute leukemia patients were resistant to at least 3 antibiotics that belong to different antimicrobial categories and to which the strain does not have intrinsic resistance (MRE definition indicated by Magiorakos et al. (80)). Thus, all strains that were identified were indeed multidrug resistant. The prevalence of resistances to different antimicrobials in strains isolated from acute leukemia patients during this study is represented in Table R1. Importantly, the majority of the isolated MRE strains are susceptible to amikacin. Amikacin belongs to the group of aminoglycosides, and it is often used in combination with tigecycline, showing promising efficacy both in vitro and in vivo in the critically ill host (81). Interestingly, the prevalence of resistance to tigecycline is low among MRE strains analyzed in this study, except for the strains that belong to the genus Enterobacter, which in $48 \%$ of the cases are resistant to this antibiotic. Nevertheless, for those infections produced by Echerichia or Citrobacter strains isolated from this study, the combination of amikacin and tigecycline treatment would be a good choice. On the other hand, the prevalence of the MRE strains resistant to ciprofloxacin, an antibiotic that 
belongs to the group of fluoroquinolones and is used as prophylaxis in acute leukemia patients, was high. Furthermore, the percentage of MRE strains isolated in this study and resistant to cefuroxime (second generation beta-lactam antibiotic) or cefotaxime (extended spectrum beta-lactamase antibiotic) is extremely high, confirming that the majority of the isolated strains, besides being MRE, are ESBL-producing Enterobacteriaceae. 
Results

Table R1. Percentages of MRE strains resistant to different antimicrobials and isolated from acute leukemia patients.

\begin{tabular}{|c|c|c|c|c|c|c|c|c|c|c|c|c|c|c|c|c|c|c|}
\hline 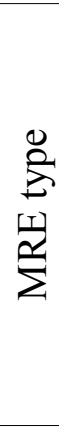 & $\begin{array}{l}. \Xi \\
. \bar{\Xi} \\
. \bar{Z} \\
\stackrel{Z}{Z}\end{array}$ & 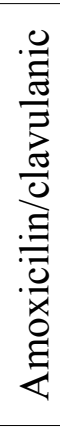 & 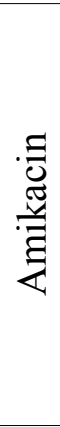 & 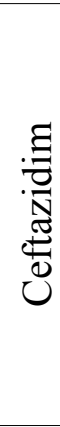 & 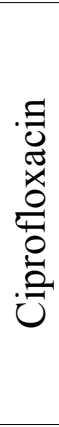 & 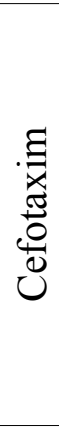 & 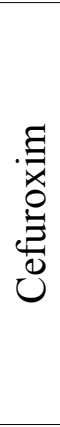 & 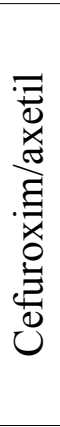 & 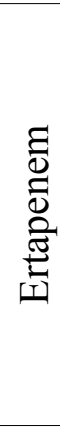 & 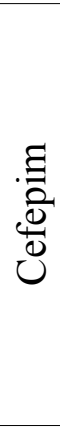 & 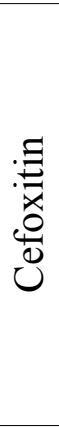 & 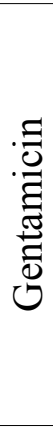 & 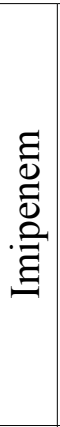 & 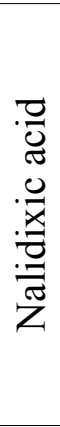 & 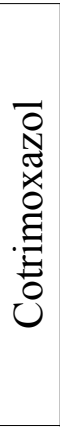 & 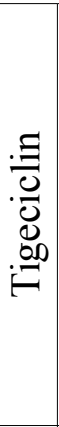 & 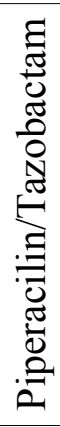 & 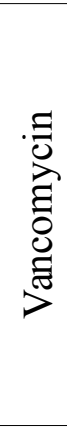 \\
\hline 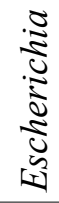 & $\frac{ \pm}{\Delta}$ & $\frac{0}{i}$ & $\vec{r}$ & 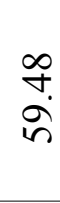 & $\frac{\AA}{\infty}$ & $\frac{0}{\infty}$ & $\begin{array}{l}\text { ָ̇ } \\
\text { ă }\end{array}$ & 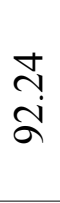 & ?ొ & 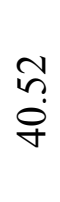 & 商 & $\stackrel{0}{\infty}$ & $\vec{n}$ & $\frac{0}{\hat{\sigma}}$ & $\stackrel{\substack{n \\
\infty}}{\infty}$ & 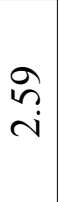 & $\begin{array}{l}n \\
n \\
0 \\
q\end{array}$ & $\begin{array}{l}8 \\
\dot{8} \\
8\end{array}$ \\
\hline 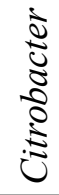 & $\begin{array}{l}8 \\
8 \\
\dot{8}\end{array}$ & $\begin{array}{l}8 \\
8 \\
8 \\
8\end{array}$ & $\begin{array}{l}\hat{\sigma} \\
\dot{\nabla}\end{array}$ & $\begin{array}{l}\hat{\sigma} \\
\hat{a}\end{array}$ & 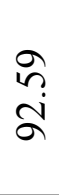 & $\begin{array}{l}8 \\
8 \\
8 \\
8\end{array}$ & $\begin{array}{l}8 \\
8 \\
8 \\
8\end{array}$ & $\begin{array}{l}8 \\
\stackrel{8}{8} \\
\stackrel{8}{0}\end{array}$ & $\hat{n}$ & 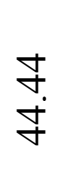 & $\begin{array}{l}\hat{\sigma} \\
\dot{a}\end{array}$ & $\begin{array}{l}\text { กิ } \\
\text { in }\end{array}$ & 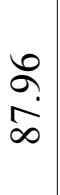 & $\begin{array}{l}\hat{\sigma} \\
\dot{a}\end{array}$ & $\begin{array}{l}n \\
n \\
\infty \\
0\end{array}$ & 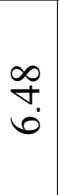 & $\stackrel{\vec{\nabla}}{\stackrel{\nabla}{\infty}}$ & \&. \\
\hline $\begin{array}{l}\vec{\Xi} \\
: \vec{\Xi} \\
\stackrel{\Xi}{\Xi} \\
\vec{v}\end{array}$ & $\begin{array}{l}\stackrel{8}{0} \\
\dot{8} \\
\stackrel{0}{2}\end{array}$ & $\frac{0}{\infty}$ & $\begin{array}{l}\text { ț } \\
\stackrel{0}{ }\end{array}$ & $\stackrel{n}{a}$ & $\frac{\hat{\sigma}}{\infty}$ & $\begin{array}{l}\infty \\
\text { ळa }\end{array}$ & $\begin{array}{l}8 \\
8 \\
8 \\
8\end{array}$ & $\begin{array}{l}8 \\
8 \\
8 \\
\stackrel{8}{0}\end{array}$ & $\begin{array}{l}\text { J } \\
\text { in }\end{array}$ & $\frac{n}{\stackrel{n}{\sigma}}$ & 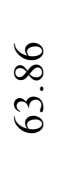 & $\stackrel{\mathbb{2}}{\stackrel{\infty}{\infty}}$ & $\begin{array}{l}\bar{n} \\
\stackrel{0}{0} \\
\sim\end{array}$ & $\stackrel{+}{\stackrel{+}{+}}$ & $\underset{\infty}{\infty}$ & $\begin{array}{l}\hat{b} \\
\vec{\sim}\end{array}$ & $\frac{m}{\ddot{\infty}}$ & $\begin{array}{l}8 \\
\dot{8}\end{array}$ \\
\hline 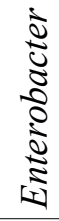 & $\begin{array}{l}8 \\
\stackrel{8}{8} \\
\stackrel{0}{ }\end{array}$ & $\begin{array}{l}8 \\
\stackrel{8}{8} \\
\stackrel{0}{0}\end{array}$ & $\begin{array}{l}8 \\
8 \\
+\end{array}$ & $\begin{array}{l}8 \\
\stackrel{8}{8} \\
\stackrel{0}{0}\end{array}$ & $\begin{array}{l}8 \\
\text { in } \\
\text { nิ }\end{array}$ & $\begin{array}{l}8 \\
8 \\
8 \\
8\end{array}$ & $\begin{array}{l}8 \\
8 \\
8 \\
8\end{array}$ & $\begin{array}{l}8 \\
\stackrel{8}{8} \\
\stackrel{8}{8}\end{array}$ & $\begin{array}{l}8 \\
\dot{0} \\
0\end{array}$ & $\begin{array}{l}8 \\
\stackrel{\infty}{\infty}\end{array}$ & $\begin{array}{l}8 \\
\dot{8}\end{array}$ & $\begin{array}{l}8 \\
\text { in } \\
\text { ñ }\end{array}$ & $\begin{array}{l}8 \\
\dot{+} \\
\end{array}$ & $\begin{array}{l}8 \\
0 \\
\infty \\
0\end{array}$ & $\begin{array}{l}8 \\
\stackrel{0}{0} \\
i n\end{array}$ & $\begin{array}{l}8 \\
0 \\
\infty \\
+\end{array}$ & $\begin{array}{l}8 \\
\text { ì }\end{array}$ & $\begin{array}{l}8 \\
\dot{8} \\
8\end{array}$ \\
\hline 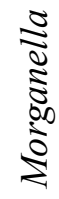 & $\begin{array}{l}8 \\
\circ \\
\stackrel{8}{0}\end{array}$ & $\begin{array}{l}8 \\
8 \\
8 \\
8\end{array}$ & 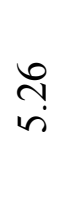 & $\frac{6}{6}$ & $\begin{array}{l}8 \\
8 \\
8 \\
8\end{array}$ & $\begin{array}{l}8 \\
8 \\
8 \\
8\end{array}$ & $\frac{\mathbb{T}}{\dot{\sigma}}$ & 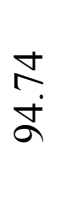 & $\stackrel{n}{n}$ & $\begin{array}{l}n \\
\infty \\
\infty\end{array}$ & $\begin{array}{l}\text { กె } \\
\text { ֻे }\end{array}$ & $\underset{\infty}{\tilde{\sigma}}$ & $\begin{array}{l}n \\
\vdots \\
0\end{array}$ & $\begin{array}{l}8 \\
8 \\
8\end{array}$ & $\begin{array}{l}\bar{\sim} \\
\dot{\infty}\end{array}$ & $\begin{array}{l}\stackrel{0}{ } \\
\text { in } \\
i\end{array}$ & 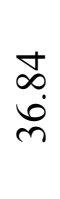 & $\begin{array}{l}8 \\
8 \\
8\end{array}$ \\
\hline 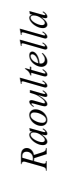 & $\begin{array}{l}8 \\
\dot{8} \\
\dot{8}\end{array}$ & $\begin{array}{l}8 \\
8 \\
8 \\
8\end{array}$ & $\stackrel{8}{8}$ & $\begin{array}{l}8 \\
\stackrel{\infty}{0}\end{array}$ & $\begin{array}{l}8 \\
8 \\
8 \\
8\end{array}$ & $\begin{array}{l}8 \\
8 \\
8 \\
8\end{array}$ & $\begin{array}{l}8 \\
8 \\
8 \\
8\end{array}$ & $\begin{array}{l}8 \\
\stackrel{8}{8} \\
\stackrel{8}{ }\end{array}$ & $\begin{array}{l}8 \\
8 \\
8 \\
8\end{array}$ & $\begin{array}{l}8 \\
8 \\
8 \\
8\end{array}$ & $\begin{array}{l}8 \\
\stackrel{8}{8} \\
\stackrel{0}{0}\end{array}$ & ○. & $\begin{array}{l}8 \\
\stackrel{8}{8} \\
\varnothing\end{array}$ & $\begin{array}{l}8 \\
8 \\
8\end{array}$ & $\begin{array}{l}8 \\
8 \\
8 \\
8\end{array}$ & $\begin{array}{l}8 \\
0\end{array}$ & $\begin{array}{l}8 \\
8 \\
8 \\
8\end{array}$ & $\begin{array}{l}8 \\
8 \\
8\end{array}$ \\
\hline $\begin{array}{r}0 \\
0 \\
0 \\
0 \\
0 \\
0\end{array}$ & $\begin{array}{l}8 \\
\stackrel{8}{8} \\
ᄋ\end{array}$ & $\begin{array}{l}8 \\
8 \\
8\end{array}$ & $\begin{array}{l}8 \\
0\end{array}$ & $\stackrel{m}{m}$ & $\stackrel{8}{8}$ & $\begin{array}{l}8 \\
8 \\
8\end{array}$ & $\begin{array}{l}8 \\
8 \\
8 \\
8\end{array}$ & $\begin{array}{l}8 \\
8 \\
8\end{array}$ & $\underset{0}{8}$ & $\stackrel{m}{m}$ & $\stackrel{8}{8}$ & $\stackrel{8}{8}$ & $\begin{array}{l}8 \\
8 \\
8 \\
8\end{array}$ & $\begin{array}{l}8 \\
8\end{array}$ & $\begin{array}{l}8 \\
0\end{array}$ & \begin{tabular}{l}
8 \\
\hdashline
\end{tabular} & $\begin{array}{l}8 \\
0\end{array}$ & $\begin{array}{l}8 \\
8 \\
8\end{array}$ \\
\hline 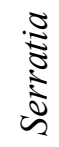 & $\begin{array}{l}8 \\
\dot{8} \\
\varnothing\end{array}$ & $\begin{array}{l}8 \\
8 \\
8\end{array}$ & $\stackrel{0}{0}$ & $\begin{array}{l}8 \\
0 \\
0\end{array}$ & : & . & $\begin{array}{l}8 \\
8 \\
8 \\
8\end{array}$ & $\begin{array}{l}8 \\
8 \\
8\end{array}$ & \&. & $\stackrel{8}{8}$ & $\begin{array}{l}8 \\
8 \\
8\end{array}$ & $\stackrel{8}{0}$ & $\begin{array}{l}8 \\
\dot{8}\end{array}$ & $\stackrel{8}{8}$ & $\stackrel{8}{8}$ & $\begin{array}{l}8 \\
0 \\
0\end{array}$ & $\stackrel{8}{8}$ & $\begin{array}{l}8 \\
8 \\
8\end{array}$ \\
\hline
\end{tabular}


During the study period, fecal samples were collected from 140 acute leukemia patients in total (Figure R15A). The number of patients positive to MRE at least once was $84(60 \%)$, while $56(40 \%)$ patients never got colonized with an MRE. We collected 817 samples, out of which 227 (27.78\%) were positive to MRE while 590 (72.22\%) were negative (Figure R15B). The most frequently isolated MRE belonged to genus Escherichia (31.39\% of positive samples). The other most predominant MRE were Citrobacter (28.83\%), Klebsiella (25.91\%), Enterobacter (6.93\%), Morganella (4.74\%), Raoultella (1.09\%), Proteus (0.73\%). In addition, Serratia was detected in 1 sample $(0.36 \%)$ (Figure R15C).

A

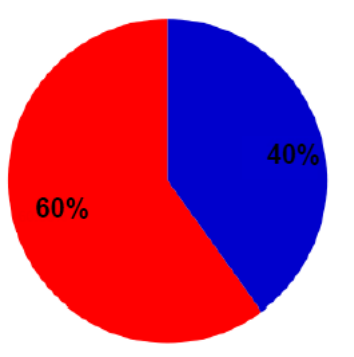

Negative
B

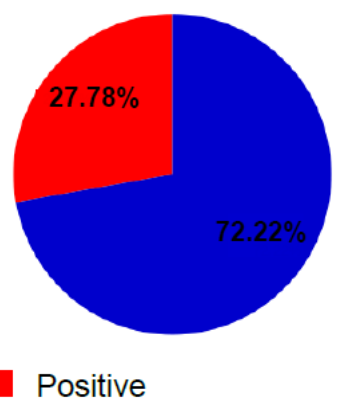

C

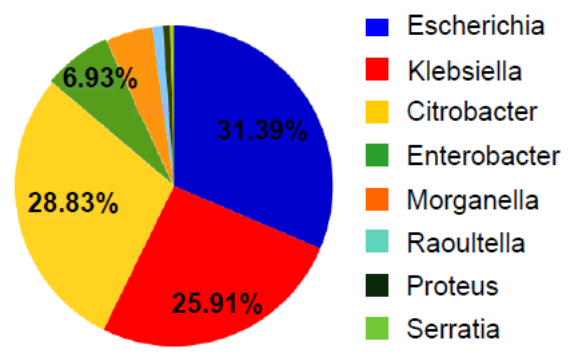

Figure R15. Prevalence of MRE positive patients (A), samples (B) and MRE types (C) in this study.

\subsubsection{Non-microbiota variables associated with MRE intestinal levels}

In the previous chapter, we have defined commensal species that may be key for conferring protection against MRE using a mouse model. Experiments with mice allow the control of other variables, beside the microbiota, that could impact MRE colonization levels (e.g. all mice have the same immune status, gender,...). However, studies in patients cannot be controlled to such extent, and other variables, besides the microbiota, that could impact MRE intestinal colonization should be also taken into account. For this reason, we first studied how different variables (including clinical variables) could affect MRE intestinal colonization levels. For this purpose, clinically relevant data such as immune status of the patient, antibiotics received, underlying diseases, etc. were collected for each analyzed patient. In addition, other data that could impact MRE intestinal colonization (i.e. gender, age) were also taken into account. All variables 
analyzed could be divided in 2 groups. The first group is composed of those variables that do not vary with time (gender, type of leukemia patient suffered, age, type of MRE detected in the sample, type of admission- if the sample was collected during admission during which chemotherapy or transplant were performed). Temporal variables were included in the second group of clinical factors (antibiotic treatment, neutropenia: low number of neutrophils, parenteral nutrition: intravenous nutrition in which the normal process of digestion is bypassed, mucositis: inflammation of the oral and gastrointestinal mucosa, antifungal treatment). Due to the different nature of the studied variables, analysis of their effect on MRE intestinal colonization levels was performed differently for temporal and non-temporal variables, as will be explained below.

\subsubsection{Impact of non-temporal variables on MRE intestinal levels}

For the analysis of the effect of non-temporal variables all MRE positive samples $(\mathrm{N}=227)$ were grouped into categories defined by a particular variable (e.g. gender: samples were divided in those collected from male or female) (Figure R16). Wilcoxon test revealed that there were no significant differences in the levels of MRE colonization in males compared to females (Figure R16A). In order to investigate if the age of the patient at the moment of sampling could be associated with MRE colonization levels, linear regression analysis was performed. There was no significant association between the MRE levels and the age of the patients (Figure R16B). As previously described in Methods, patients included in this study were diagnosed with acute leukemia, which could be divided in 2 groups depending on the immune cells affected by the disease: lymphoblastic or myeloid. Patients with myeloid leukemia had slightly higher intestinal levels of MRE, although the observed difference was not statistically significant (Wilcoxon test $p=0.08$, Figure R16C). Since it was possible that not every MRE taxonomic type was able to colonize the intestine at same level, Kruskal-Wallis test was performed on the levels of colonization of different taxonomic types of MRE. Only MRE that were detected in at least 5 samples were included in this analysis (i.e. Escherichia, Citrobacter, Klebsiella, Enterobacter, Morganella). The analysis revealed no differences in the intestinal colonization levels between the different MRE types 
identified, suggesting that all MRE types are equally capable of colonizing the intestinal tract (Figure R16D).

A

Gender

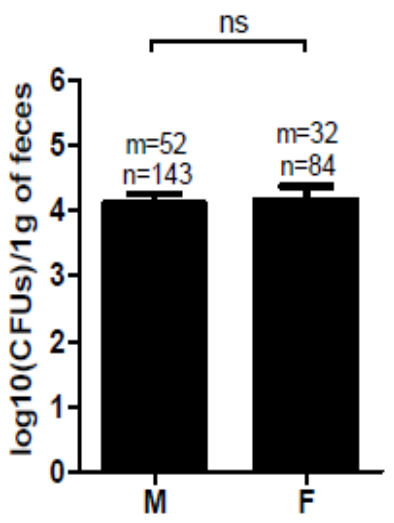

Type of leukemia

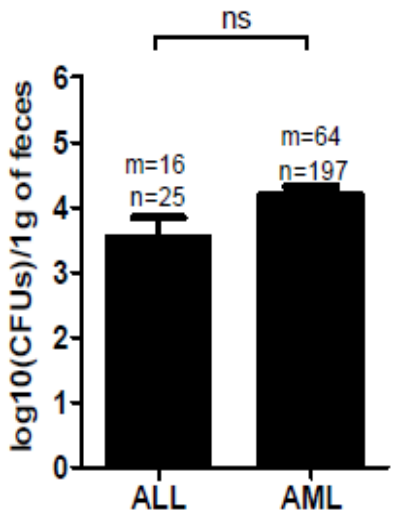

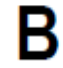

Age

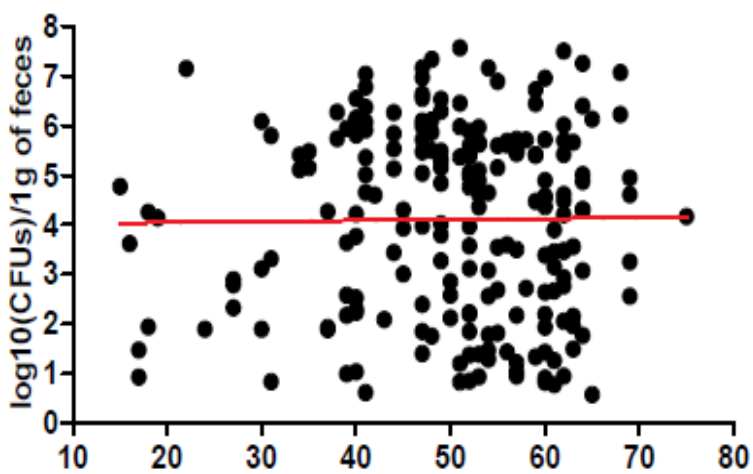

Type of MRE

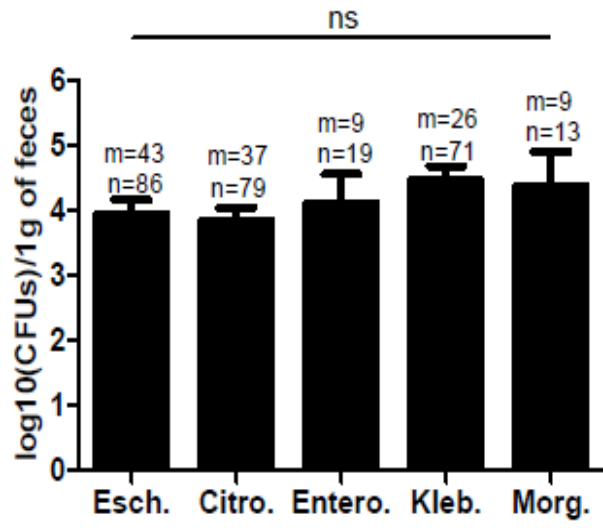

Figure R16. Effect of gender, age, type of leukemia and type of MRE on MRE colonization levels. (A) The levels of MRE in males (M) and females (F) was not statistically different (Wilcoxon test, ns-not significant). (B) Linear regression on MRE levels depending on age (years) of the patient at the moment of sampling revealed no association between MRE levels and patient's age. (C) The levels of MRE in samples collected from patients with lymphoblastic leukemia (ALL) are slightly lower but not significantly different (Wilcoxon, ns) from those measured in samples collected from patients with myeloid leukemia (AML). (D) MRE levels depending on taxonomic type of MRE detected in the sample (Escherichia- Esch., Citrobacter- Citro., Enterobacter- Entero., Klebsiella- Kleb., Morganella- Morg.). Only MRE types detected in more than 5 samples are represented. Kruskal-Wallis revealed no differences between different groups (ns). Levels of colonization are represented in logarithmic scale per gram of fecal material. Mean and $+/$ - SEM are plotted. Numbers above each bar stand for number of samples (n) and number of patients from which samples were collected $(\mathrm{m})$. 
As mentioned before, due to their health status, patients are spending long time intervals in the hospital where they undergo 1 or more rounds of chemotherapy and some of them also receive a transplant. The chemotherapy is initiated immediately after the admission to the hospital, which is why all samples collected during this admission are grouped together and analyzed as a non-temporal variable. For this reason, levels of MRE in samples collected during chemotherapy were compared to those in samples collected during the transplant period. Interestingly, Wilcoxon test revealed significantly different MRE colonization levels between these 2 groups of samples (Figure R17A). Since patients received different types of chemotherapy, which could have an effect on the levels of MRE colonization, we analyzed separately those samples obtained from patients receiving an intensive chemotherapy (results in a neutropenic state of the patient that is expected to last for more than 2 weeks) or non-intensive chemotherapy. Interestingly, levels of MRE collected during intensive chemotherapy were similar to the levels of MRE collected during the non-intensive one, suggesting that the type of chemotherapy that the patient received does not affect MRE colonization levels (Figure R17B). On the other hand, transplant treatment could be allogeneic or autologous depending on the source of the transplanted cells (the donor and recipient of the cells are the same person- autologous, or a different person- allogeneic). Man-Whitney revealed that the type of transplant did not influence the MRE intestinal levels (Figure R17C).
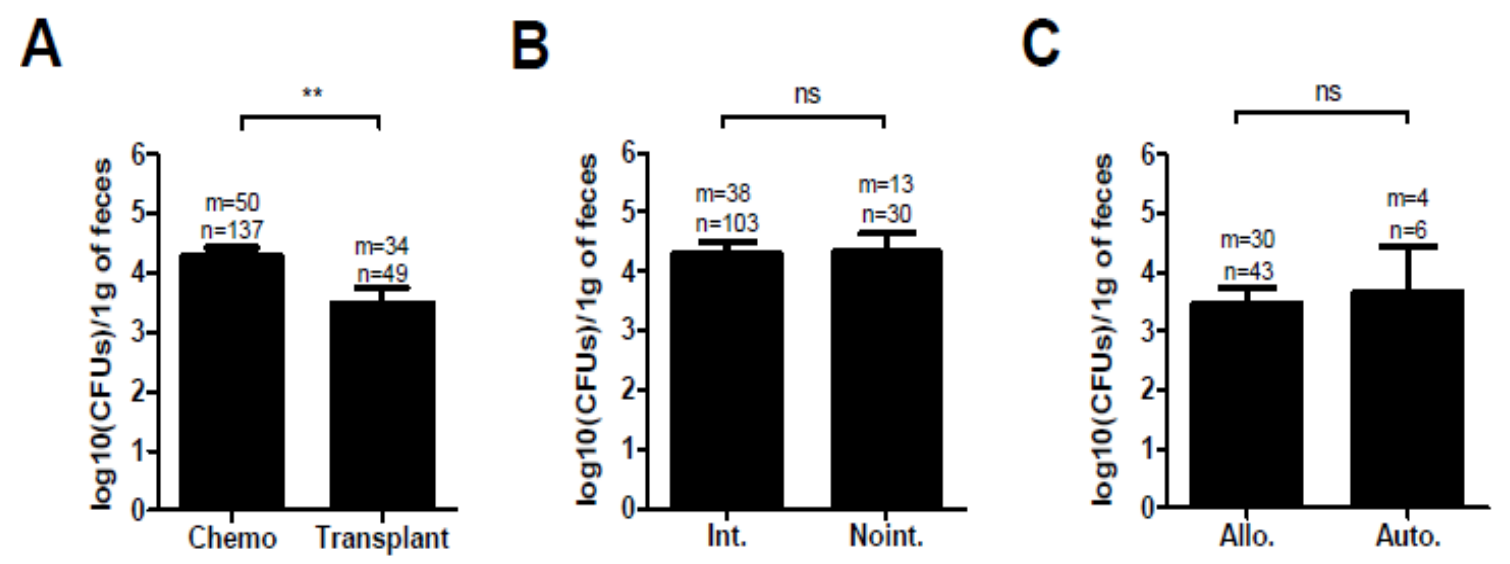

Figure R17. Effect of chemotherapy and transplant on MRE intestinal colonization levels. (A) MRE fecal levels in samples collected during an admission in which the patient received chemotherapy (Chemo) differed significantly from MRE levels in samples collected during an admission in which the patient received a transplant (Transplant), (Wilcoxon test, ${ }^{* *} \mathrm{p}<0.01$ ). (B) Wilcoxon test revealed no differences 
(ns-not significant) in MRE levels due to the intensity of the chemotherapy received. (C) The type of transplant (allogeneic or autologous) does not impact MRE intestinal levels (Wilcoxon test, ns). Levels of colonization are represented in logarithmic scale per gram of fecal material. Mean and +/- SEM are plotted. Numbers above each bar stand for number of samples (n) or number of patients from which samples were collected (m).

\subsubsection{Impact of temporal variables on MRE intestinal levels}

As previously mentioned, variables such as neutropenia or antibiotic treatment are initiated at some point of patient's admission and last for different periods of time. For this reason, these variables were considered as temporal. In order to investigate the effect of these variables on MRE colonization levels, paired analysis was performed. For this purpose, levels of MRE in the sample previous to the initiation of the treatment or complication (i.e. mucositis) were compared to the levels of MRE in the following sample collected after the initiation of the treatment/complication, both samples being collected during the same admission. This analysis allowed us to investigate if certain treatments or conditions could be associated with increase or decrease in MRE colonization levels once the patient has been colonized. Clinical variables whose effects on MRE colonization levels were tested through Wilcoxon matched-pairs signed rank test are neutropenia, parenteral nutrition, mucositis, antifungal treatment and antibiotic treatment. Interestingly, analysis revealed that decrease in the number of neutrophils was associated with significant decrease in MRE levels (Wilcoxon matched-pairs signed rank test $\mathrm{p}<0.05$, Figure R18A), while parenteral nutrition, mucositis or any of the tested antifungal treatments seem not to affect MRE levels, once the MRE has colonized the intestinal tract (Wilcoxon matched-pairs signed rank test, Figure R18B-E). 
A

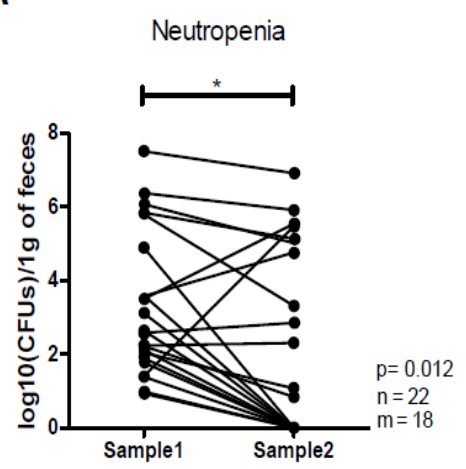

B

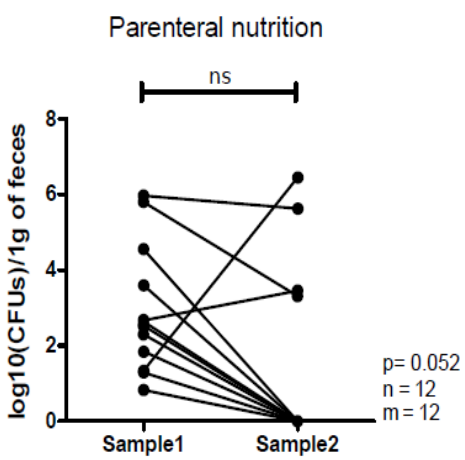

C

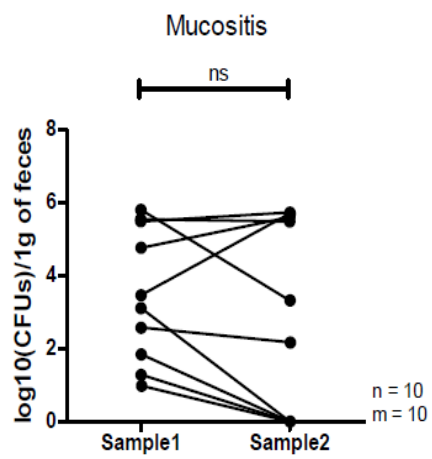

D

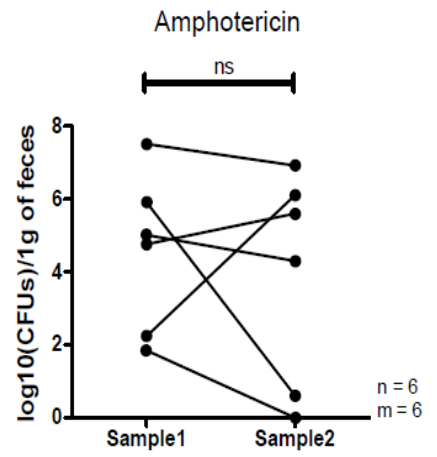

E

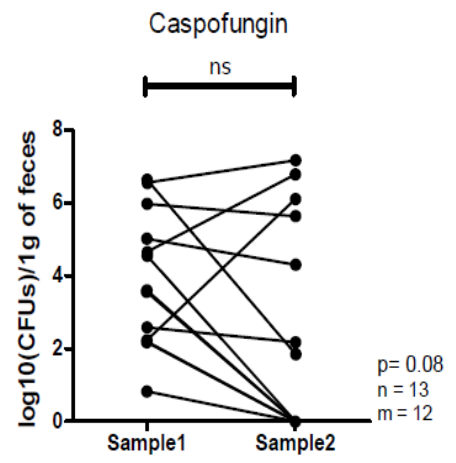

Figure R18. Levels of MRE in samples collected before and after the initiation of the clinical variables. (A) Wilcoxon matched-pairs signed rank test revealed significant $\left({ }^{*} p<0.05\right)$ decrease in MRE levels upon initiation of the neutropenic state in patients. Other clinical variables such as parenteral nutrition (B), mucositis (C), amphotericin treatment (D), and caspofungin treatment (E) do not affect MRE levels (ns-not significant), although in case of parenteral nutrition and caspofungin treatment, $p$ value of Wilcoxon matched-pairs signed rank test was lower than 0.1 , and is stated next to the graph. Levels of colonization are represented in logarithmic scale per gram of fecal material. Numbers next to each graph stand for number of pairs of samples (n) or number of patients from which samples were collected (m).

A similar analysis was performed to investigate the effect of different antibiotic treatments on MRE intestinal levels. For this purpose, antibiotic treatments for which more than 5 pairs of samples existed (aminoglycosides, beta-lactams and glycopeptides) were considered for the analysis. Antibiotics were arranged into different classes in order to obtain more strength for the statistic analysis. The class of beta-lactams included aztreonam, meropenem and piperacillin/tazobactam, while teicoplanin and vancomycin composed the class of glycopeptides. Aminoglycosides included amikacin treatment. Interestingly, analysis of the effect of different antibiotic treatments on MRE colonization levels revealed that beta-lactams (Wilcoxon matched-pairs signed rank test, 
$\mathrm{p}<0.05)$ and glycopeptides $(\mathrm{p}<0.01)$ decrease MRE levels (Figure R19), while aminoglycosides do not have an effect on the MRE colonization levels.

A

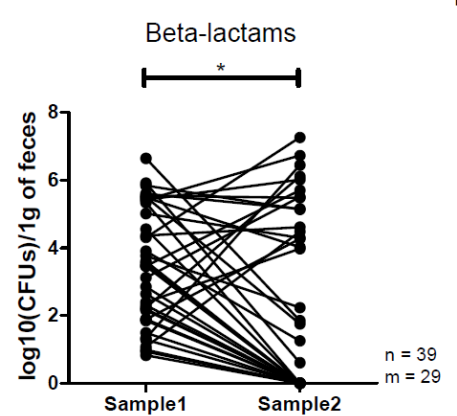

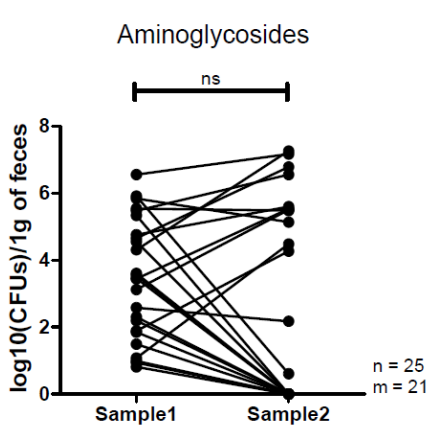

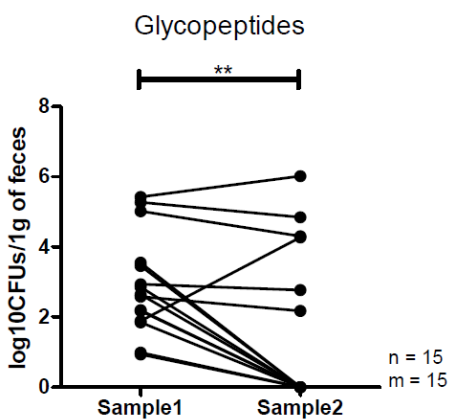

Figure R19. Levels of MRE in samples collected before and after the initiation of different antibiotic treatments. (A) Wilcoxon matched-pairs signed rank test revealed significantly lower levels of MRE in samples collected after the introduction of beta-lactams to patients $\left({ }^{*} \mathrm{p}<0.05\right)$. (B) No significant changes were observed in MRE levels upon administration of aminoglycosides (ns-not significant). (C) Glycopeptide treatment decreased MRE levels significantly $(* * p<0.01)$. Levels of colonization are represented in logarithmic scale per gram of fecal material. Numbers next to each graph stand for number of pairs of samples (n) or number of patients from which samples were collected (m).

Results represented in Figure R19 suggest that antibiotic treatment, although given intravenously, which is the case for these antibiotics, could reach the intestine and have effect on MRE directly or through changes in the intestinal microbiota. Importantly, some of the strains detected in the samples that were included in this analysis are susceptible to some of the analyzed antibiotics, while others are resistant. For this reason, paired analysis for each antibiotic treatment was repeated, taking into account the data about sensitivity of the MRE strain to the given antibiotic. Analysis of the effect of betalactams on MRE levels in a group of samples in which the MRE detected strains were sensitive to the beta-lactams revealed that this antibiotic decreased significantly MRE colonization levels (Wilcoxon matched-pairs signed rank test $p<0.001$; Figure R20A). The observed reduction was more clear compared to when all pairs of samples were analyzed, independently if MRE was sensitive or resistant to this antibiotic (Figure R19A). Since MRE strains besides sensitivity to beta-lactams, could also display susceptibility to other antibiotics that were given to the patient during the same time period, the analysis was repeated, but in this case only samples in which MRE strains were sensitive to beta-lactams, and not to any other antibiotic given during the time of 
sampling were included in the Wilcoxon matched-pairs signed rank test (Figure R20B). Since there were only 3 pairs of samples that fulfilled the criteria, it was not possible to obtain a significant $\mathrm{p}$ value using the Wilcoxon test. Nevertheless, in all 3 pairs of samples, administration of beta-lactam resulted in a complete clearance of MRE, suggesting that beta-lactams produce a reduction in MRE colonization levels when the MRE is exclusively susceptible to this antibiotic and not to other antibiotics that were received by the patients during the sampling period. On the other hand, it is possible that the antibiotic treatment could promote MRE colonization levels, when MRE is not sensitive to the given antibiotic. For this reason, an analysis was performed to determine if beta-lactams could increase MRE colonization levels when they were given to patients colonized with an MRE strain resistant to the beta-lactams received (Figure R20C). Interestingly, there were no significant changes in MRE colonization levels in these pairs of samples. Thus, beta-lactams does not seem to promote intestinal colonization by MRE. Although the MRE detected in samples from this last analysis were resistant to beta-lactams, they could be sensitive to other antibiotics given at the same time, which would mask the effect of beta-lactams. For this reason, pairs of samples containing MRE sensitive to other antibiotics given during the same period as beta-lactams were eliminated for the subsequent analysis (Figure R20D). Interestingly, the result was similar to the one obtained before: beta-lactams do not seem to increase MRE colonization levels when they are given to patients who are colonized with MRE strains resistant to the received beta-lactams. 
A

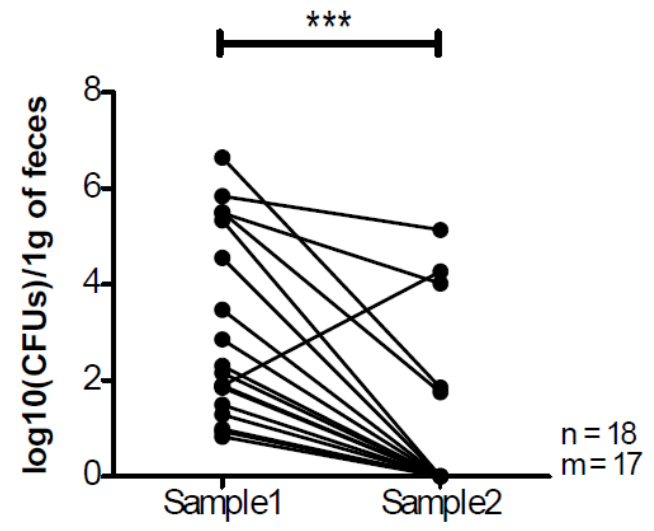

C

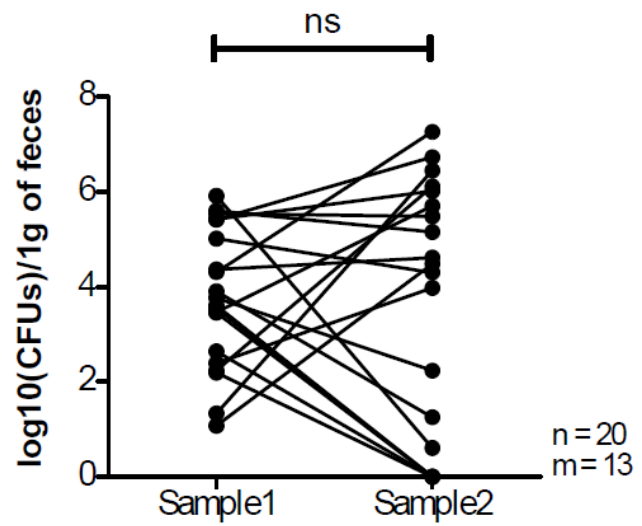

B

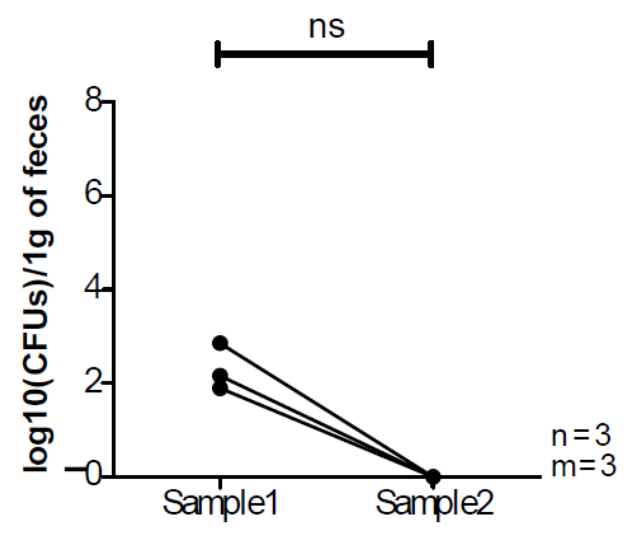

D

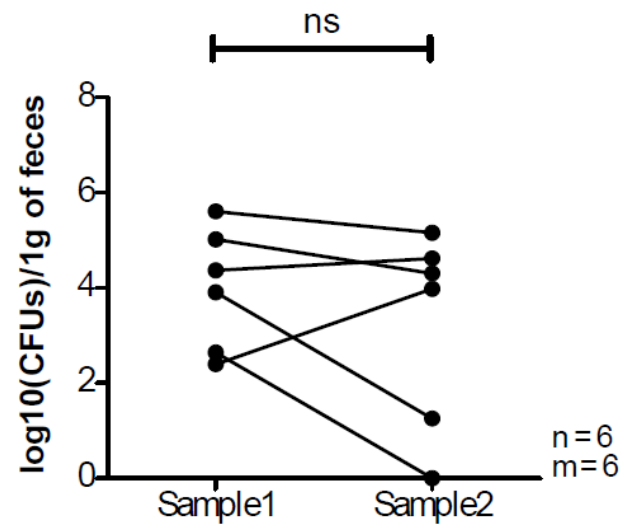

Figure R20. Levels of MRE in fecal samples collected before and after the administration of betalactams. (A) When only those pairs of samples in which the identified MRE is sensitive to beta-lactams are included in analysis, the reduction in MRE levels upon beta-lactam treatment was even higher compared to analysis when all pairs of samples, independently if MRE strain was sensitive to the betalactams, were included in analysis $(* * p<0.001)$. (B) When only are included those pairs of samples with MRE sensitive to beta-lactams but resistant to all other antibiotics given during that period of time, the decrease in MRE colonization levels was not significant, probably due to the low number of pairs of samples $(\mathrm{N}=3)$, although the trend in MRE reduction was clear. (C) Analysis did not reveal change in MRE levels when beta-lactams were given to patients who were colonized with MRE strains resistant to this antibiotic (ns-not significant). (D) The same result was obtained when from the group of samples shown in $\mathrm{C}$, were eliminated those samples that contained an MRE sensitive to other antibiotics received during the period of sampling. Levels of colonization are represented in logarithmic scale per gram of fecal material. Numbers next to each graph stand for number of samples (n) or number of patients from which samples were collected (m).

A similar analysis was performed to identify the effect of aminoglycosides on MRE colonization levels. Although there was no overall change in MRE levels when 
aminoglycosides were administered to patients (Figure R19B), it was of interest to test the effect of this treatment on samples with MRE strains sensitive to this antibiotic (Figure R21A). The analysis revealed there was significant reduction in MRE levels in these pairs of samples (Wilcoxon matched-pairs test, $p=0.043$ ), suggesting that systemic aminoglycosides also have an inhibitory effect against intestinal MRE that are sensitive to this antibiotic. As in the analysis with the beta-lactams, and in order to investigate the effect of exclusively aminoglycosides on MRE, those pairs of samples, in which MRE is sensitive to other antibiotics also received by the patient during the sampling period, were excluded from the analysis (Figure R21B). This last analysis shown that intravenous aminoglycosides alone also have an inhibitory effect on MRE intestinal levels when they are given to patients colonized with strains sensitive to these antibiotics, since in all 5 cases the MRE was completely cleared after aminoglycoside administration (Wilcoxon matched-pairs test, $p=0.031)$. Due to the low number of samples $(n=2)$, it was not possible to analyze the effect of aminoglycosides when they were given to patients colonized with aminoglycosides-resistant MRE strains.

A

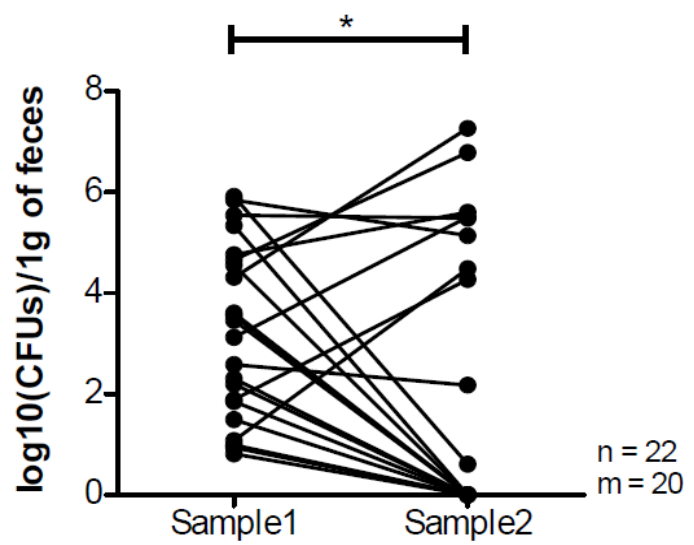

B

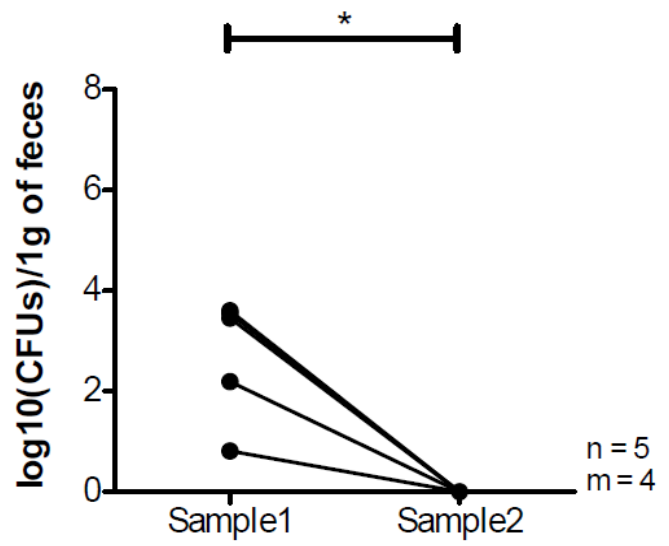

Figure R21. Levels of MRE in samples collected before and after the administration of aminoglycosides. (A) Wilcoxon matched-pairs signed rank test revealed that when only are included those pairs of samples in which the detected MRE was sensitive to aminoglycosides, the reduction was significant $(p=0.043)$. (B) When only are included those pairs of samples with MRE sensitive to aminoglycosides but resistant to all other antibiotics given during the sampling period, the decrease in MRE colonization levels was even more pronounced $(\mathrm{p}=0.031)$. Levels of colonization are represented in logarithmic scale per gram of fecal material. Numbers next to each graph stand for number of pairs of samples (n) or number of patients from which samples were collected (m). 
Analysis performed in order to investigate the effect of glycopeptides treatment on MRE levels revealed that these antibiotics decreased MRE levels (Wilcoxon matchedpairs signed rank test $p<0.01$; Figure R19C). These results were somewhat unexpected since glycopeptides have no effect on Gram-negative bacteria such as MRE. In order to investigate if this was due to the effect of another antibiotic given at the time, additional analysis were performed. As in case with other antibiotic treatments, Wilcoxon matchedpairs signed rank test was applied on pairs of samples collected before and after the initiation of glycopeptide treatment, in which MRE was resistant to all other antibiotics given during the sampling period (Figure R22). In this case, only 3 pairs of samples fulfilled the conditions. Although glycopeptide administration seem to decrease MRE levels, due to the low number of samples, the observed change was not statistically significant.

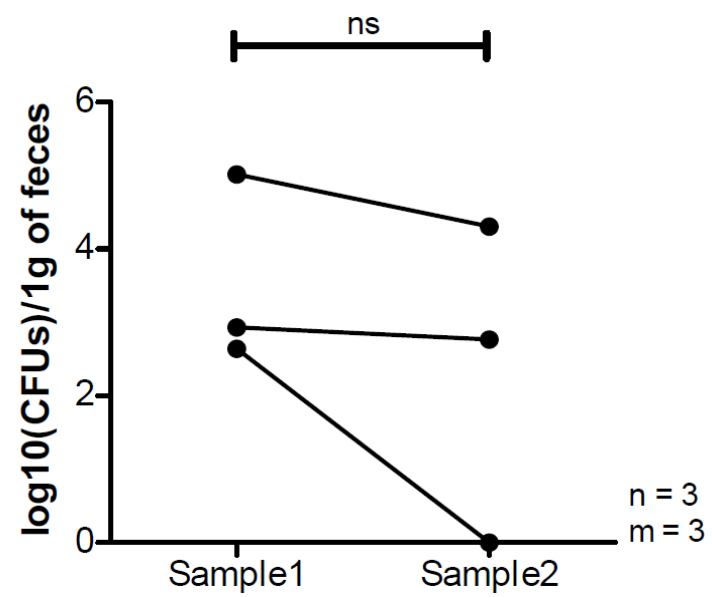

Figure R22. Levels of MRE in samples collected before and after the administration of glycopeptides. When only those pairs of samples in which the detected MRE was resistant to all other antibiotics given during the sampling period were included in analysis, the detected changes were not significant (ns-not significant), although the trend towards MRE reduction could be observed $(p=0.125)$. Levels of colonization are represented in logarithmic scale per gram of fecal material. Numbers next to each graph stand for number of pairs of samples (n) or number of patients from which samples were collected (m).

Results of the analysis performed in order to investigate association between antibiotic treatment and MRE colonization levels revealed that antibiotics given intravenously to which MRE strains are sensitive could reach the intestine and decrease 
MRE levels. Moreover, it seems that these antibiotics, when administered to patients colonized with resistant MRE strains do not promote MRE colonization levels. Due to the effect of antibiotics on sensitive strains, analysis performed on other temporal clinical variables (neutropenia, mucositis, parenteral nutrition and antifungal treatment) were repeated, this time eliminating those pairs of samples in which the MRE strains were sensitive to antibiotics given during the sampling period. Due to the low number of samples, analysis was performed only for parenteral nutrition and neutropenia. Importantly, also in this new analysis, initiation of neutropenia seems to reduce the levels of MRE ( $p=0.034$, Figure R23A). These results indicate that, unexpectedly, neutropenia may have an inhibitory effect on MRE intestinal levels. On the other hand, parenteral nutrition does not seem to affect MRE intestinal levels ( $p=0.25$, Figure R23B).

A

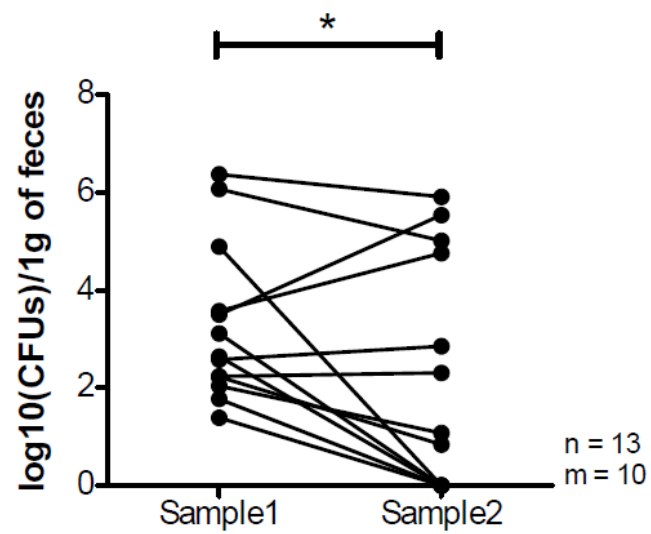

B

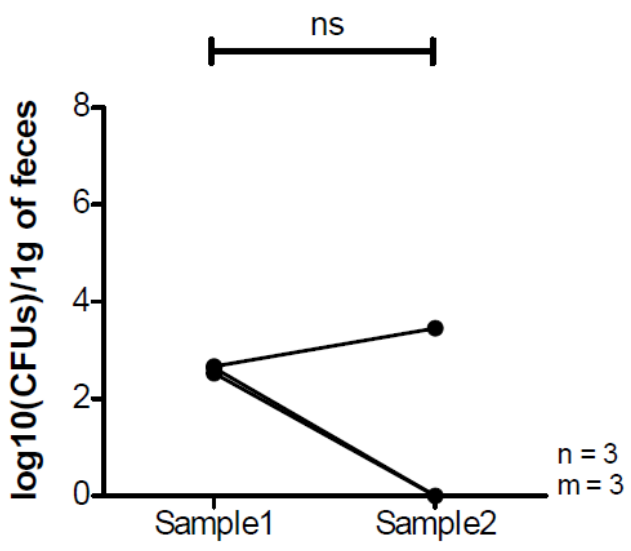

Figure R23. Levels of MRE in samples collected before and after the initiation of neutropenia and parenteral nutrition. (A) Analysis performed exclusively on those pairs of samples with MRE strains resistant to all antibiotics received during the sampling period revealed a reduction of MRE levels upon initiation of the neutropenic state $(p=0.034)$. (B) When analyzed only those pairs of samples with MRE strains resistant to all antibiotics received during the sampling period, parenteral nutrition does not seem to affect MRE intestinal levels ( $p=0.25$ ). Levels of colonization are represented in logarithmic scale per gram of fecal material. Numbers next to each graph stand for number of samples (n) or number of patients from which samples were collected (m).

In order to study the independent effect of clinical variables on MRE colonization levels, all clinical variables were included in a multivariate analysis. For this purpose, Lasso regression with positive penalty term $\lambda$ was applied, as explained in Methods. This approach in combination with a suitable model evaluation that included 
cross validation reduced the risk of overfitting the final model. The response variable in the model was the change in MRE colonization levels between 2 consecutive samples. Data about the treatment or condition that started in the period between 2 paired samples were included as predictor variables. Besides, data about gender, type of leukemia or type of admission were also added as predictors of the model. Lasso regression resulted in the selection of 2 variables for the model that can explain changes in MRE levels of colonization ( $p=6.54 \mathrm{E}-05$, Figure R24). These 2 variables are administration of betalactams when MRE is susceptible to the beta-lactams and colonization with Klebsiella. Sensitivity to beta-lactams when these are administered affects negatively MRE levels, while colonization with Klebsiella affects positively. In previous analysis, Kruskal-Wallis showed no differences in MRE intestinal levels between different taxonomic types (Figure R16D). However, a trend towards higher MRE levels in case taxonomic type was Klebsiella could be actually observed. Moreover, Wilcoxon test revealed significantly higher MRE levels between group of samples in which the identified MRE was Klebsiella as compared to those in which the detected MRE was Citrobacter $(\mathrm{p}<0.05)$. Importantly, with only these 2 variables, sensitivity to beta-lactams and colonization with Klebsiella, the model generated by Lasso regression is able to predict $12.5 \%$ of the variability in the MRE levels data from this study $\left(\mathrm{R}^{2}\right.$ adjusted for the model is 0.125$)$.

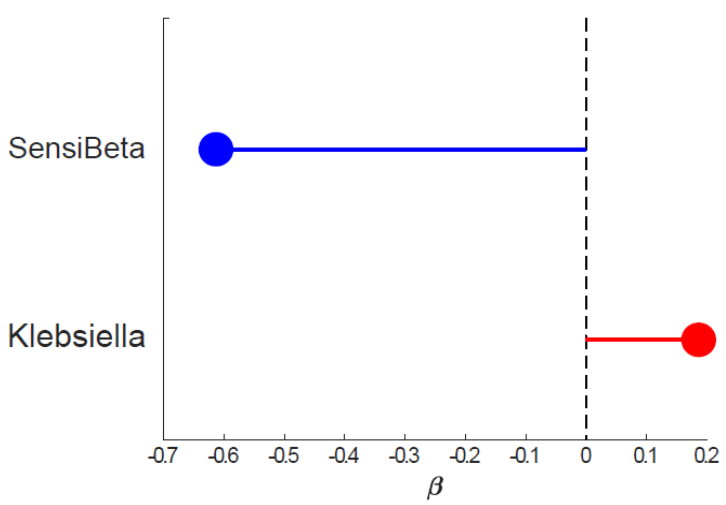

Figure R24. Clinical variables selected with multivariate analysis as predictors for change in MRE levels. Sensitivity to beta-lactams in MRE strains when these antibiotics are given to patients correlates negatively with MRE levels, while colonization with Klebsiella correlates positively to MRE colonization levels. The $\mathrm{x}$ axis represents coefficients of interaction $(\beta)$ for these 2 clinical variables. 


\subsubsection{Clinical parameters associated with the intestinal colonization levels in the first MRE positive sample}

Previous analysis revealed clinical variables associated with changes in MRE levels once the patient is colonized. Additional analysis were performed in order to investigate if any clinical variable, previous to the colonization with MRE, could influence MRE intestinal colonization levels. For this purpose, colonization levels in the first MRE positive sample from each admission were analyzed. In order to assure that the patient got colonized in the hospital, samples included in the analysis had to be preceded with an MRE negative sample. Due to the results obtained from previous analysis in which administration of antibiotics to which MRE strains were sensitive resulted in reduction of colonization levels, all samples that were susceptible to antibiotics that were given to the patient at the moment of sampling were discarded from the analysis. Only those clinical variables that had more than 5 samples per group were analyzed. Analysis revealed that the occurrence of mucositis previous to the first MRE colonization resulted in trend towards higher levels of MRE. Although the comparison was not statistically significant, $p$ value was low (Wilcoxon test $p=0.057$; Figure R25A). It was possible that the effect of mucositis on MRE colonization levels could depend on the time that passed since mucositis until MRE colonization (probably mucositis has a greater effect on MRE levels if it has occurred close to the time-point when MRE was detected). For this reason, linear regression was applied in order to take into consideration the time from the initiation of mucositis to the moment in which MRE was detected. Linear regression analysis confirmed our hypotheses: levels of colonization in the first MRE positive sample were higher when mucositis occurred shortly before the sampling (Figure R25B). Moreover when the comparison represented in Figure R25A was repeated with only those cases in which mucositis occurred no more than 2 weeks previous to MRE detection, the difference in MRE levels between samples collected after the occurrence of mucositis and MRE levels in samples from patients that did not develop mucositis was statistically significant (Wilcoxon test $\mathrm{p}<0.05$; Figure R25C). 

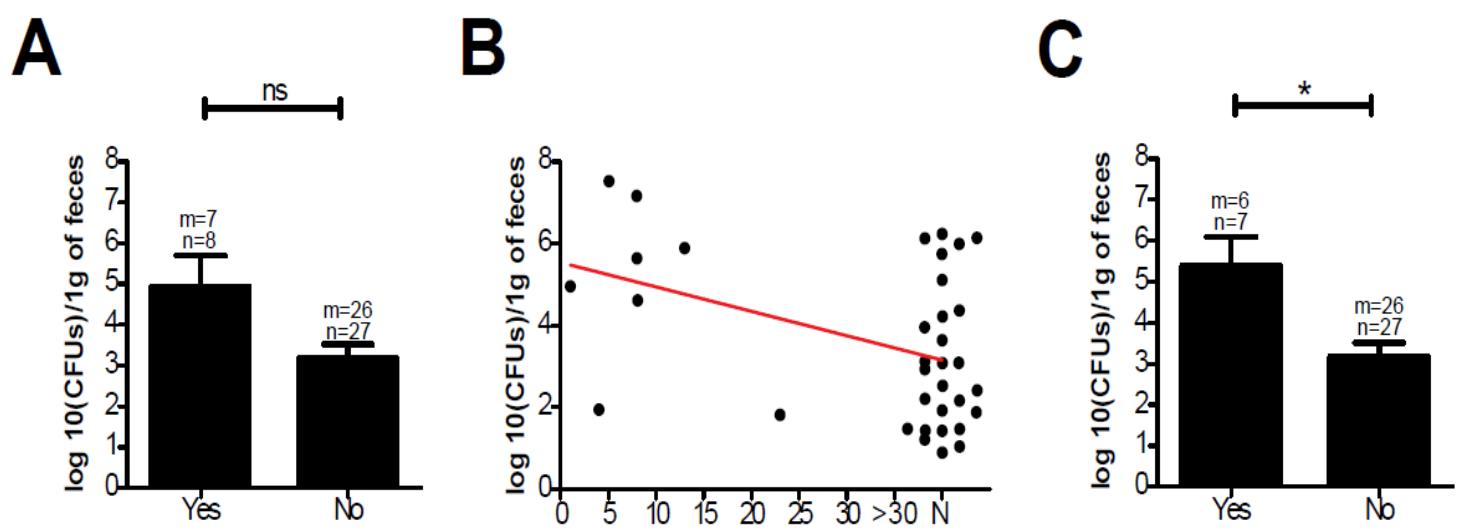

Figure R25. Effect of mucositis on MRE intestinal levels in the first MRE positive sample identified during the patient's hospitalization period. (A) Levels of MRE intestinal colonization in the first MRE positive sample from each hospital admission when patients developed mucositis previously (Yes) or when mucositis did not occur before sampling during the same admission period (No). Wilcoxon test revealed no significant differences between these 2 groups, although the $p$ value was close to significant $(p=0.057)$. (B) MRE levels in the first positive sample in relation with the days that passed since mucositis ( $\mathrm{x}$ axis). All samples collected more than 1 month after mucositis are grouped together $(>30)$. Samples from patients that never developed mucositis previous to colonization are also grouped together $(\mathrm{N})$. Linear regression revealed significant trend toward higher MRE levels in samples collected shortly after mucositis $(\mathrm{p}<0.05)$. (C) Wilcoxon test revealed significant differences $\left({ }^{*} \mathrm{p}<0.05\right)$ between MRE levels in the first positive sample in the group of samples collected no more than 2 weeks after mucositis (Yes) compared to MRE levels in samples collected from patients that did not develop mucositis (No). Levels of colonization are represented in logarithmic scale per gram of fecal material. Numbers next to each graph stand for number of samples (n) or number of patients from which samples were collected (m).

The same analysis was repeated with other clinical variables such as neutropenia, parenteral nutrition, antifungal and antibiotic treatment. In none of these comparisons MRE levels were significantly affected by the treatment or condition previous to the sampling (Figure R26). 
A

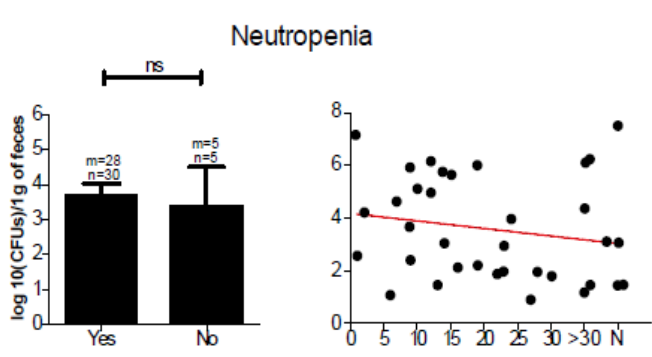

C

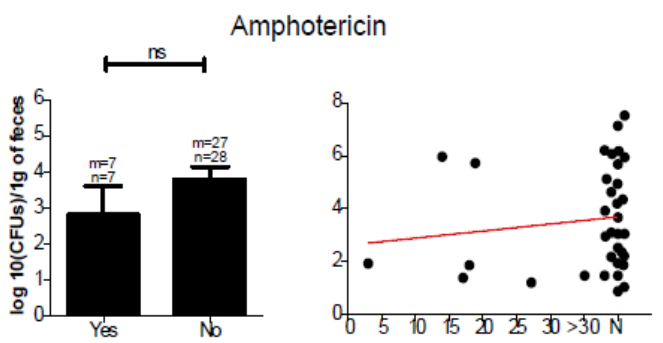

$\mathbf{E}$

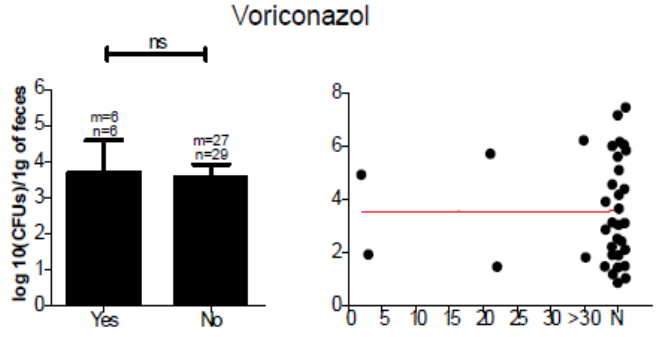

G

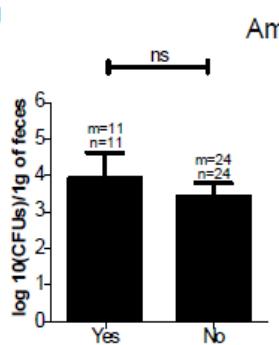

Amikacin

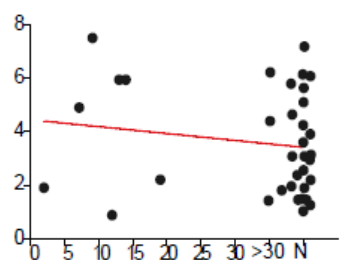

I

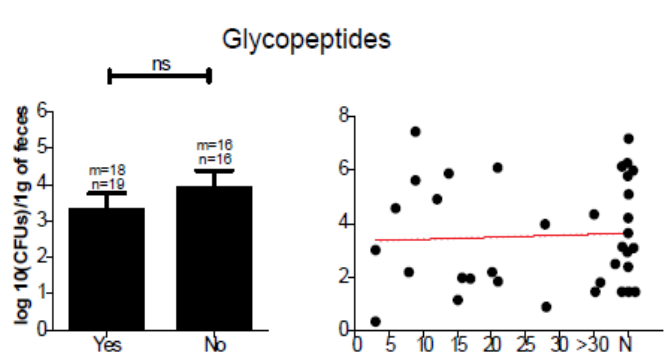

B

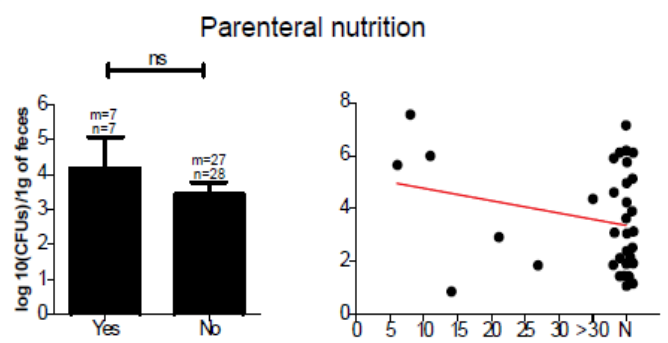

D

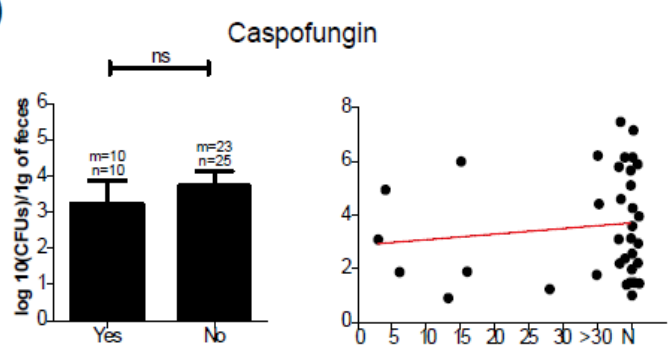

$\mathbf{F}$

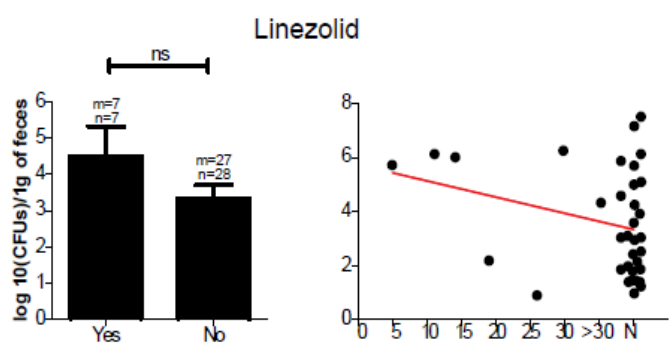

$\mathrm{H}$

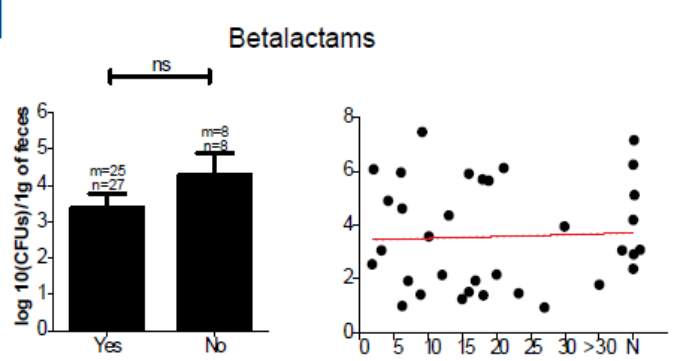

$\mathbf{J}$

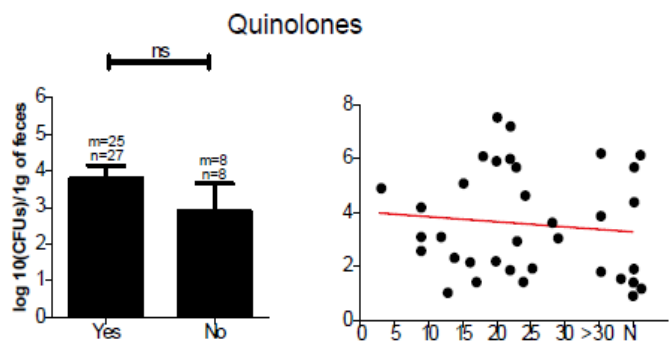

Figure R26. MRE levels in the first positive sample from each admission for all tested clinical variables. The bargraphs represent the comparison of (i) MRE levels between samples collected after the occurrence of the variable under study (condition or treatment) (Yes) with (ii) the MRE levels in samples 
collected from patients that did not have that particular treatment or condition (No). For all clinical variables shown in this figure, Wilcoxon showed no significant differences (ns-not significant). Linear regression was applied in order to investigate if MRE colonization levels could be associated with time that passed since the treatment or complication until the moment of sampling (figures on the right for each variable analyzed). None of the clinical variables shown association with MRE intestinal levels using linear regression analysis. Levels of colonization are represented in logarithmic scale per gram of fecal material. Numbers next to each graph stand for number of samples (n) or number of patients from which samples were collected $(\mathrm{m})$.

\subsubsection{Identification of bacteria associated with protection against MRE colonization in hospitalized patients}

In the previous chapter, we have defined the effect of clinical variables on MRE intestinal levels. Here, we wanted to study how changes in the microbiota composition may impact the ability of MRE to colonize the intestine. Results from this analysis could identify those commensal bacteria associated with protection against MRE (when they are present MRE levels decrease, while MRE can efficiently colonize the intestine when they are absent). In order to perform this analysis, we analyzed the composition of the microbiota of all samples obtained from every patient. An example of the data obtained from 1 patient is presented in Figure R27. As it can be observed in Figure R27, a patient can be admitted into the hospital in several occasions for several reasons (e.g chemotherapy or transplant). This particular patient got colonized with MRE in the second admission period and the MRE intestinal levels varied in time until MRE was completely eliminated in the third admission period. Different antibiotic treatments, which can have an effect on MRE levels and on the microbiota composition, are being introduced to the patient alone or in the combination with other treatments during the admission period. Moreover, although not shown in this particular example, as mentioned in the previous chapter, patient could suffer complications such as neutropenia or/and mucositis that could also affect MRE colonization levels. 

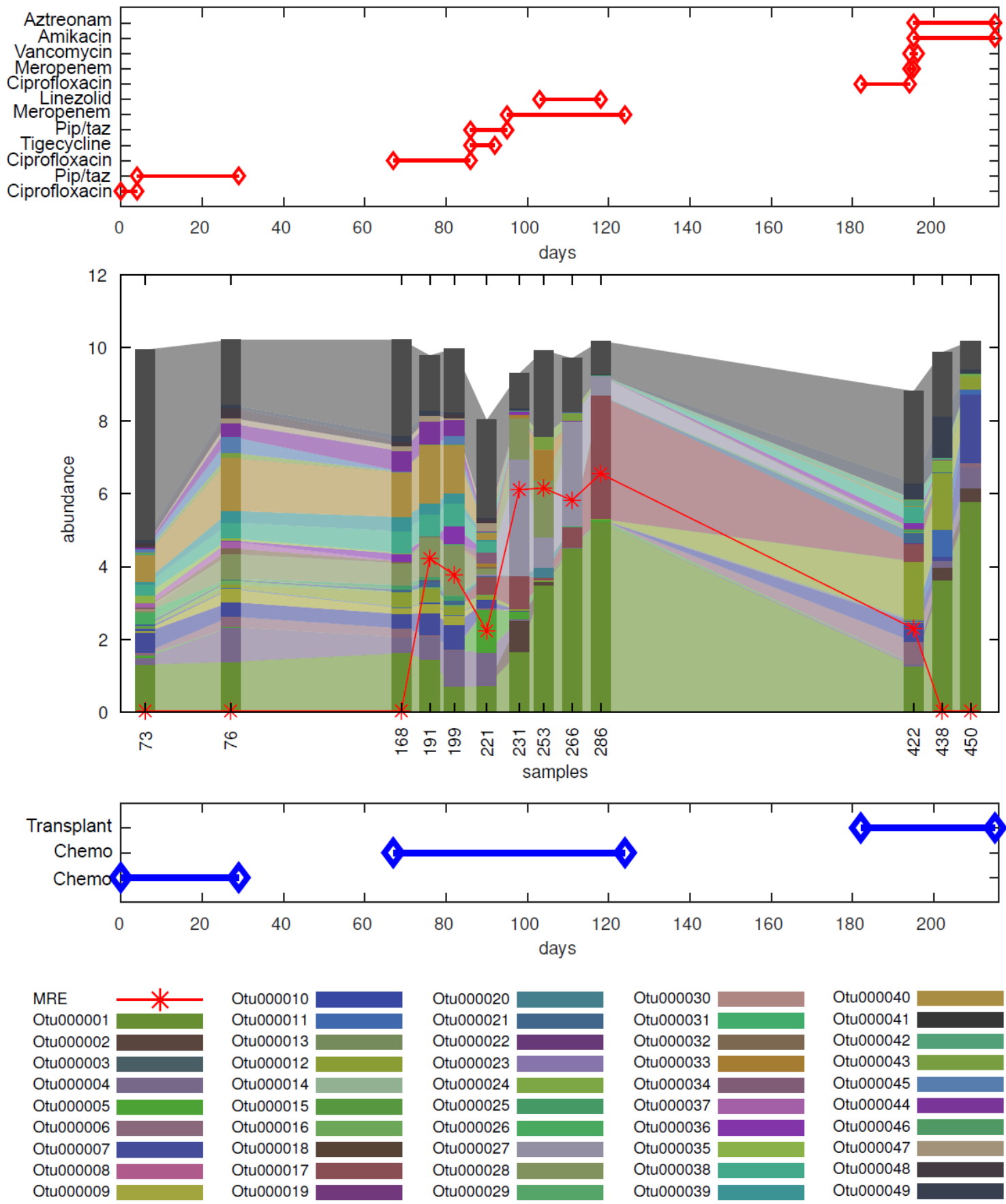

\begin{tabular}{l|l} 
Otu000030 \\
Otu000031 \\
Otu000032 \\
Otu000033 \\
Otu000034 \\
Otu000037 \\
Otu000036 \\
Otu000035 \\
Otu000038 \\
Otu000039
\end{tabular}

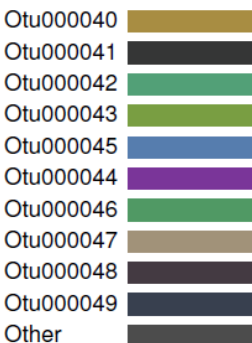

Figure R27. Microbiota composition and clinical variables collected for a particular patient. In the middle rectangle, microbiota composition of all samples collected from this patient is represented: each bar represents 1 sample. The most abundant bacterial OTUs (49 together with the group "Other" containing the remaining lower abundance OTUs) are shown and labeled with different colors as indicated. Red line represents MRE levels identified by plate counting technique. Lower rectangle represents 3 admissions during which all samples were collected. Patient went through 2 rounds of chemotherapy and subsequently 
received a transplant. Upper rectangle represents the antibiotic treatment the patient received. Other variables included in the analysis are not represented in order to simplify the graph.

Due to the complexity of the data, it would not be possible to identify commensal bacteria associated with protection against MRE colonization in acute leukemia patients with classic statistical analysis. For this reason LV mathematical model was applied (127) This model analyzes the dynamics of the microbiota composition and accounts for external perturbations such as antibiotic treatment, allowing to identify key commensals associated with pathogen colonization levels. The theory behind the model and detailed methodology are explained in section 3.10.3 of Material and Methods. Briefly, the rate of change in MRE levels between 2 consecutive samples collected from the same admission period, normalized by the number of days that passed between both samples, was used as response variable for the model. As explained in the methodology section, since the absence of MRE in negative samples could be explained by resistance to colonization or by the absence of exposure to MRE, the rate of MRE change was calculated only for those pairs of samples in which the first one had MRE levels. At the beginning of the study, we tried to perform the analysis including all pairs of samples, independently if the first sample of the pair had MRE levels, but the solution for this model was not reliable, probably due to the problem that we have pointed out. When pairs of samples in which the first one was negative were eliminated from further analysis, the solution for the model became reliable, indicating that probably the data about pairs of samples in which the first sample was negative was introducing noise into the model. The genus abundances, representing the commensal bacteria identified in acute leukemia patients, normalized by the total bacterial density of each sample were used as predictor variables for the model. For the analysis we considered 25 most abundant genera identified in all patients, including the group "Other" containing the remaining lower abundance genera. This number of genera was chosen since introduction of more than 25 genera would not allow us generation of a reliable model, due to the high number of variables included into the model. For pairs of samples of interest, geometric mean abundances were calculated from logarithmic values of normalized genus abundances. Besides bacterial abundance data, all clinical variables that were found to be statistically significant in univariate analysis explained in sections 
4.2.2.1 and 4.2.2.2 were included into the model. Also, data about the sensitivity of MRE detected in each sample to the antibiotics given at the moment of sampling were also included into the model. In total, 132 pairs of samples entered the model. As in case of multivariate analysis of clinical variables, Lasso regression was used to solve the model.

Importantly, the model selected 7 variables that were associated with MRE levels ( $p=6.42 \mathrm{E}-07$; Figure R28). Among clinical variables, administration of betalactams, when the identified MRE strain is sensitive to these antibiotics, is negatively associated with MRE levels, confirming the result obtained by multivariate analysis on clinical variables, explained in section 4.2.2. Moreover, transplant and chemotherapy were also correlated with MRE levels. Among genera, Coprobacillus was negatively associated with MRE levels, indicating that it could be a potential protector commensal against MRE colonization. On the other hand, Paraprevotella and Klebsiella were positively correlated with the MRE intestinal levels.

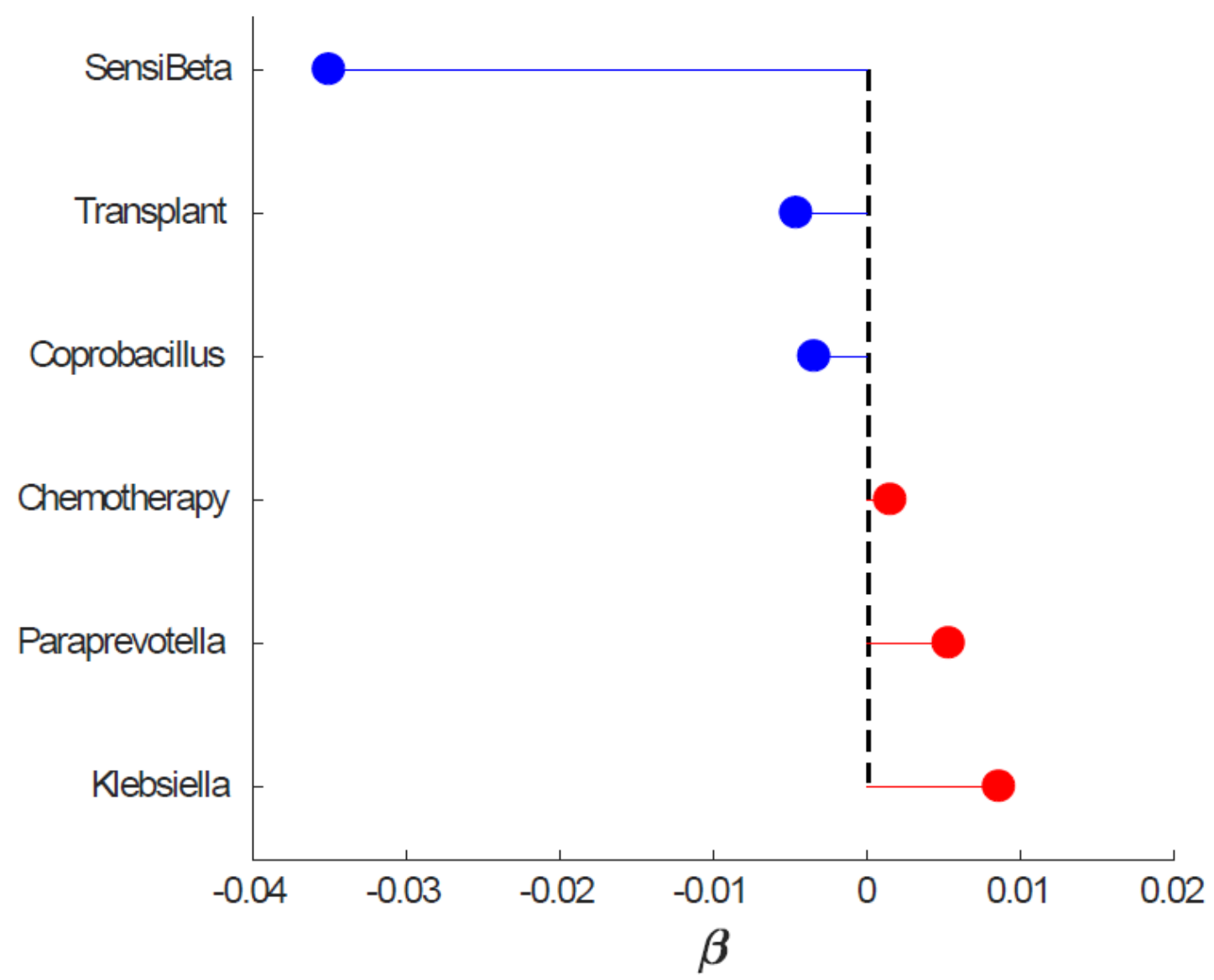

Figure R28. Genera and clinical variables selected by $L V$ as associated with MRE colonization levels. In blue are represented negatively correlated variables, while in red are those that are positively correlated 
with MRE levels. The coefficient of correlation is represented on $\mathrm{x}$ axis. Coprobacillus, administration of beta-lactams when the identified MRE strain is sensitive to beta-lactams, and transplant are negatively correlated with MRE intestinal levels. On the other hand, Paraprevotella, Klebsiella and chemotherapy are positively associated with MRE intestinal levels.

In order to visualize better the association of the genera selected by the model with MRE intestinal levels, the mean abundance of the commensal bacteria identified by the model were plotted in relation with the MRE rate (Figure R29). As shown on this figure, the abundance of Coprobacillus, the genus negatively associated with MRE levels, is higher in pairs of samples where MRE decreases, and lower in samples in which MRE increases. On the other hand, negatively associated genera (Klebsiella and Paraprevotella) are more abundant in samples in which MRE increases than in those where MRE decreases.

Negatively correlated

Coprobacillus

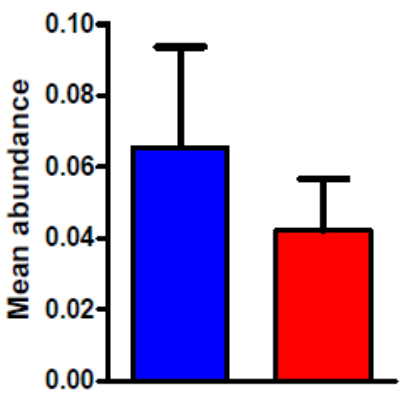

Positively correlated

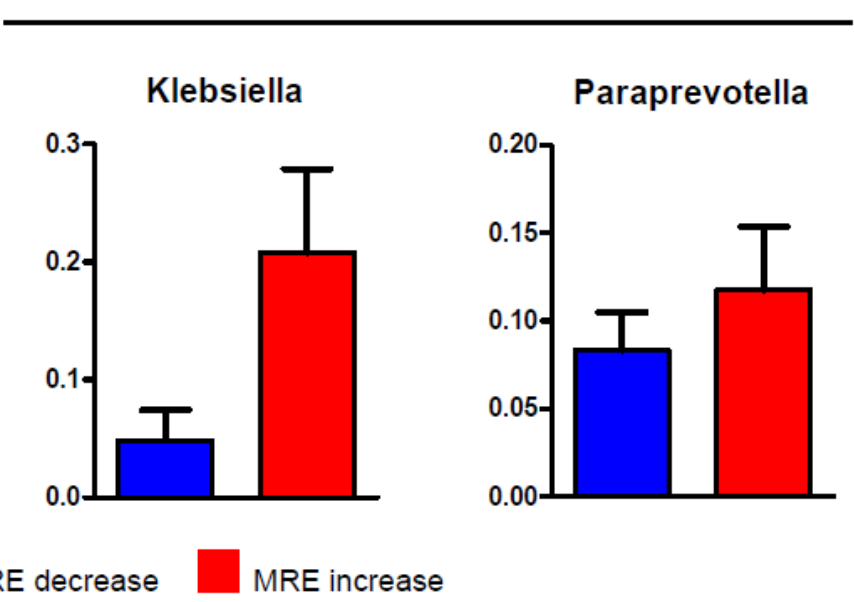

Figure R29. LV model identifies commensal bacterial genera that are associated with MRE levels in patients. Geometric mean abundance (Mean abunddance) of bacterial genera in pairs of samples in which it was observed a reduction (blue) or increase (red) of MRE. Only those bacterial genera identified by LV model to be associated with MRE intestinal levels are shown.

Since bacterial genus can be divided into different OTUs, some of which may have an effect on MRE levels and other not, and different patients may contain different OTUs belonging to the same genus, we decided to repeat the analysis, this time at the OTU level, which may allow us to identify with more precision bacteria that could have a protective role against MRE intestinal colonization. For this purpose, 25 most abundant 
OTUs (which coincide with those OTUs with the mean abundance higher than $0.1 \%$ ) were considered for analysis, including the group "Other" containing the remaining lower abundance OTUs. Importantly, the model selected 12 variables that could explain change in MRE colonization levels ( $p=7.51 \mathrm{E}-06$; Figure R30). Out of 6 bacteria selected by the model, 3 were negatively correlated with MRE levels (could have a protective effect): 1 OTU classified as Lactobacillus and other 2 as Bacteroides. Positively correlated bacteria were 3 OTUs within the genera Bacteroides, Klebsiella and Alistipes. Administration of beta-lactams in general, as well as administration when the identified MRE strain was sensitive to the beta-lactams, was negatively associated with MRE levels, which confirms the results obtained from multivariate analysis explained in section 4.2.2. Moreover, transplant (negative correlation) and chemotherapy (positive correlation) were confirmed as clinical variables that could affect MRE levels.

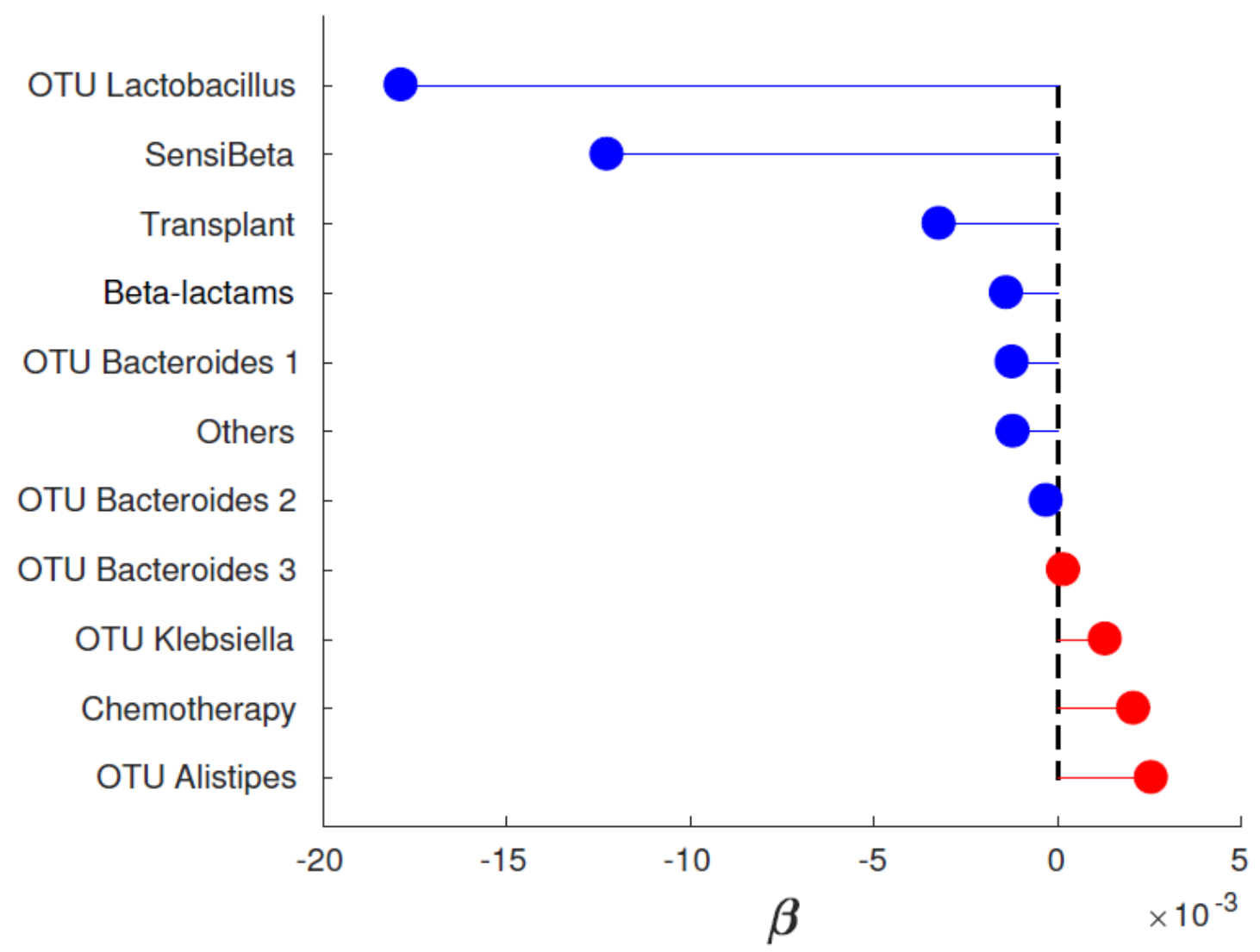

Figure R30. OTUs and clinical variables selected by $L V$ to be associated with MRE colonization levels. In blue are represented negatively correlated variables; while in red are those that are positively correlated with MRE levels. The coefficient of correlation is represented on $\mathrm{x}$ axis. Among clinical 
variables, transplant, administration of beta-lactams in general, as well as administration of beta-lactams when the detected MRE is sensitive to beta-lactams (SensiBeta) are negatively correlated to MRE. On the other hand, chemotherapy is positively associated with MRE colonization levels.

Finally, we plotted the levels of the OTUs identified by LV to be associated with MRE levels. As shown in Figure R31, all 3 OTUs that are negatively correlated with MRE levels were present in higher abundances in pairs of samples where MRE decreases, as compared to pairs of samples in which MRE levels increase, indicating that these OTUs may have a protective role against MRE. On the other hand, positively correlated bacteria were more abundant in those pairs of samples in which MRE levels increase than in those where MRE levels decrease. 


\section{Negatively correlated}

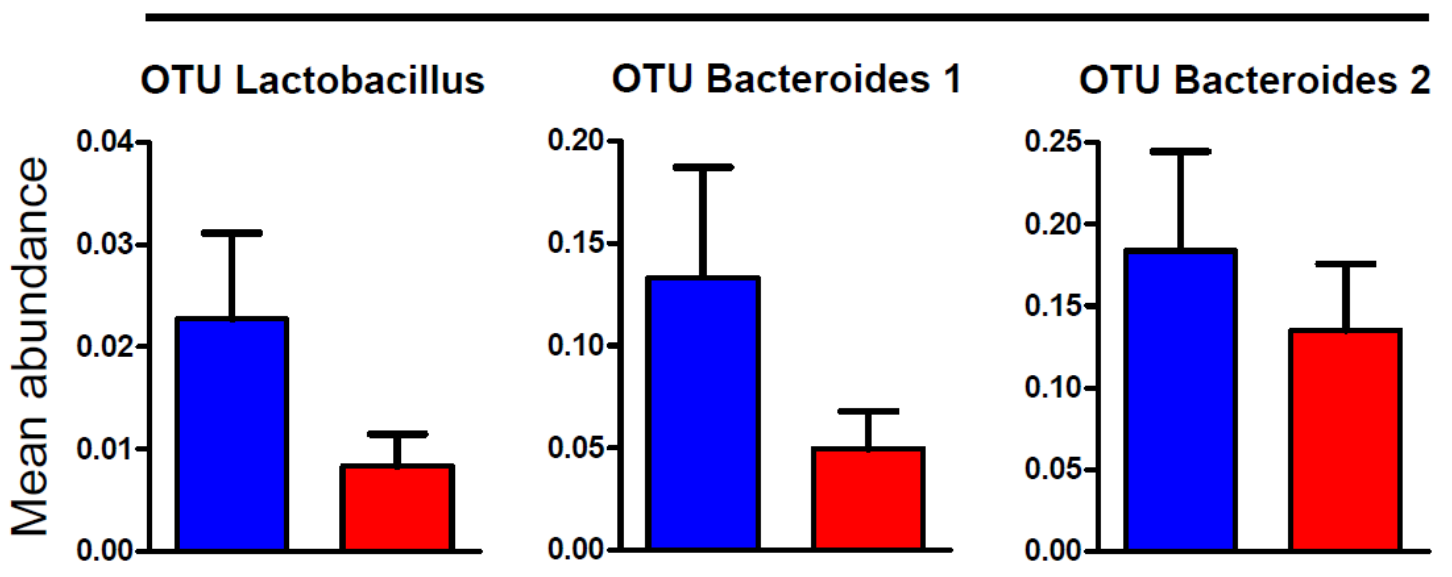

\section{Positively correlated}

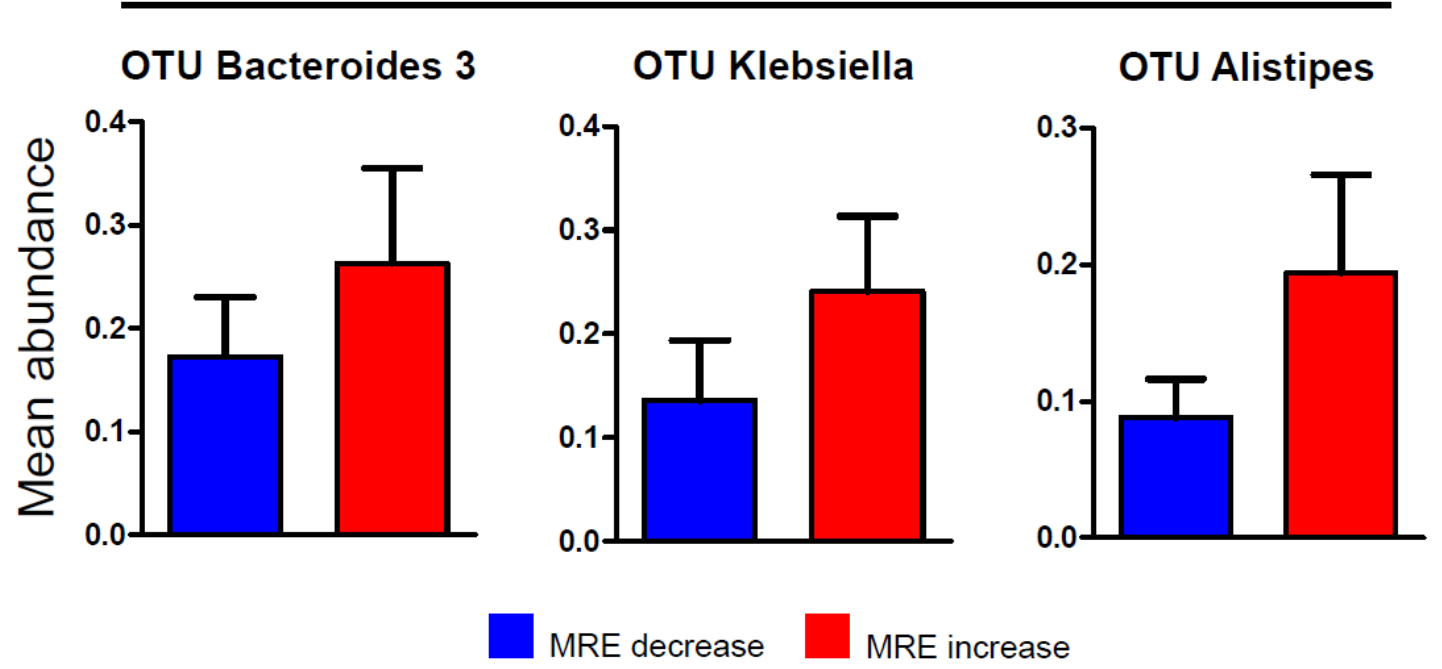

Figure R31. LV model identifies commensal bacterial OTUs that are associated with MRE levels in patients. Geometric mean abundance (Mean abundance) of bacterial genera in pairs of samples in which it was observed a reduction (blue) or increase (red) of MRE. Only those bacterial OTUs identified by the LV model to be associated with MRE intestinal levels are shown.

In order to confirm the accuracy of the model, factors selected by the models that could influence MRE levels (genera and clinical variables or OTUs and clinical variables) were used as predictors of MRE levels. Abundances of predictor genera or OTUs and presence or absence of clinical variables such as beta-lactam treatment and their coefficients of interaction with MRE served to calculate expected MRE rates for 
each pair of samples. The relation between the real MRE rates for each pair of samples and those predicted by each of the models (performed on genera or OTUs) are plotted in Figure R32. Linear regression of real against predicted MRE rates revealed that $24 \%$ of variability in MRE rates could be explained by the model that included OTU abundances as predictors $\left(\mathrm{R}^{2}\right.$ adjusted $=0.24$, Figure $\left.\mathbf{R 3 2 B}\right)$. On the other hand, the model that included 25 most abundant genera as predictors of the MRE rates was able to explain $23.9 \%$ of the variability in MRE rates $\left(\mathrm{R}^{2}\right.$ adjusted $=0.239$, Figure $\left.\mathbf{R 3 2 A}\right)$.

A

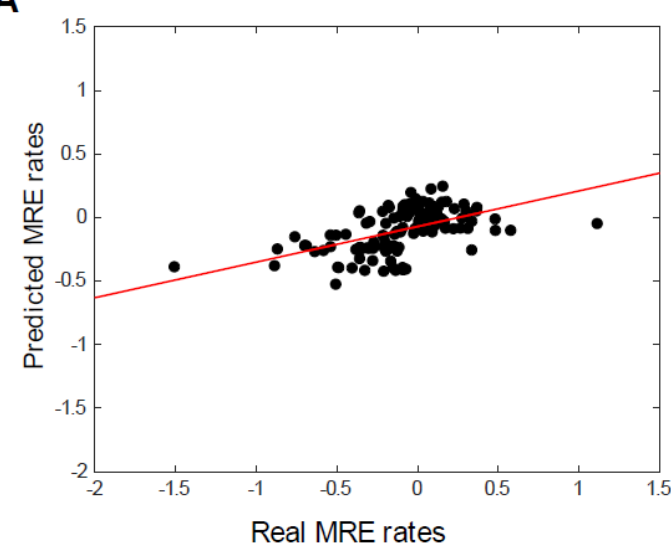

B

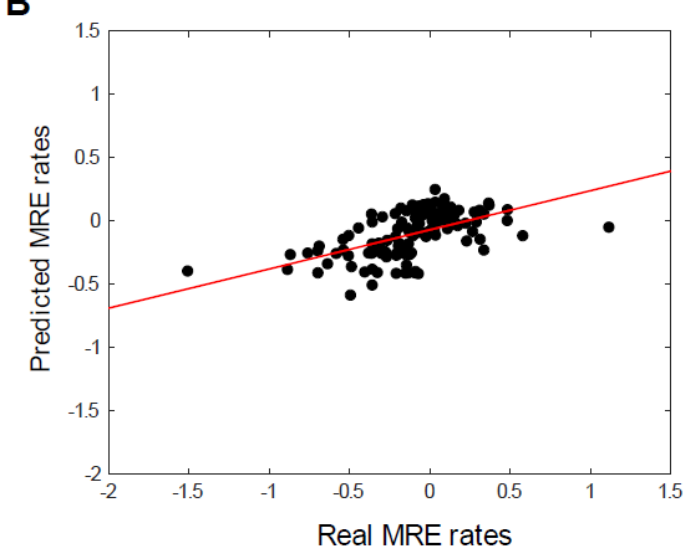

Figure R32. Real MRE rates plotted against MRE rates predicted by the models. Predicted MRE rates for pairs of samples were calculated using the variables selected by LV as correlated with MRE rates and coefficients of interaction for these variables. (A) 23.9\% of variability in MRE rates could be explained by the model that included abundances of the genera and clinical variables as predictors (linear regression, $\mathrm{R}^{2}$ adjusted $=23.9 \%$ ). (B) The model that included abundances of the OTUs and clinical variables as predictors was able to explain $24 \%$ of variability in MRE rates (linear regression, $\mathrm{R}^{2}$ adjusted= $24 \%$ ). 


\subsection{The role of the microbiota dysbiosis in ERE}

Epizootic rabbit enteropathy is a severe gastrointestinal disease that occurs in young rabbits in the first weeks post-weaning (89), a period during which changes in microbiota composition are known to occur. In a recent study, Bauerl et al. (89) described remarkable dysbiosis and reduced taxonomic diversity in cecal microbiota of animals with ERE, although it remains unclear if the observed changes are the cause or the consequence of the disease. On the other hand, Licois et al. (92) reproduced the disease in healthy animals by administrating intestinal contents obtained from rabbits affected by ERE, indicating that the disease is transmissible among rabbits and suggesting that an infectious agent may be involved in the development of this pathology. However, no causative agent has been identified for the moment. In order to investigate the role of the dysbiosis in ERE development several objectives were proposed, as previously mentioned: (i) to investigate if dysbiosis observed in ERE rabbits could be detected before the disease onset and thus could be the cause of the disease; (ii) to investigate the microbiota dynamics after the weaning and identify changes in microbiota that could lead to ERE development; (iii) to investigate if the cause of ERE could be due to an infectious agent.

\subsubsection{ERE is characterized by dysbiosis of the intestinal microbiota}

To evaluate the role of dysbiosis in ERE development, caecotroph samples were prospectively collected from littermate rabbits during 3 consecutive weeks, starting the day of weaning. Caecotrophs, soft feces made up of essentially cecal contents and secreted by rabbits at dawn, were collected instead of regular fecal samples, since the microbiota of caecotrophs is equivalent to that one from the cecum (Figure R33) and oral inoculation of cecal contents has been shown to reproduce the disease in SPF healthy rabbits (130). 


\section{Cecum}

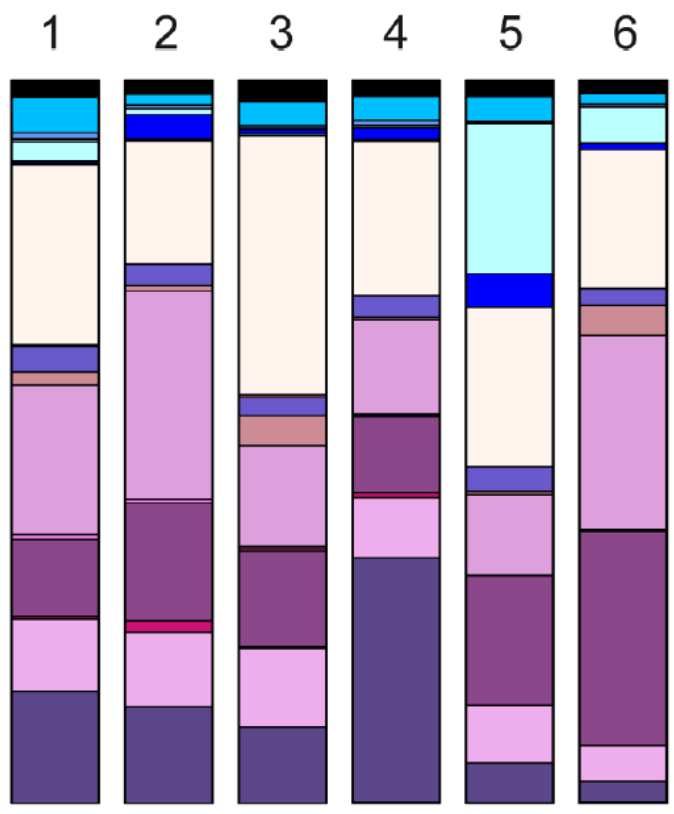

Lysinibacillus

$\square$ Bacillus

$\square$ Robinsoniella

$\square$ Roseburia

$\square$ unclassified_Lachnospiraceae

$\square$ Sporacetigenium

$\square$ Clostridium

$\square$ Sporobacter

$\square$ Subdoligranulum

$\square$ unclassified_Ruminococcaceae

$\square$ Acetivibrio

$\square$ Oscillibacter

$\square$ unclassified Clostridiales

$\square$ unclassified_Clostridia

\section{Caecotrophs}

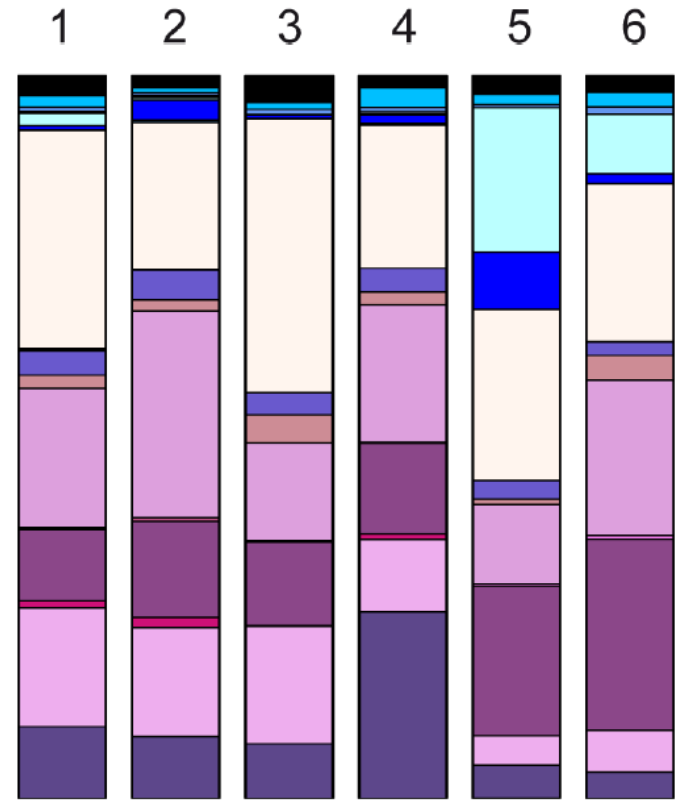

Figure R33. cecal and caecotrophs microbiota of rabbits. Phylogenetic classification of 16S rDNA frequencies in the cecum or caecotrophs samples from healthy rabbits, 21 days after weaning. Each bar represents the microbiota of an individual rabbit whose ID number is indicated above the bar. The most predominant bacterial taxa are shown and labeled with different colors as indicated. Bacterial taxa were obtained by classification of $16 \mathrm{~S}$ rDNA sequences to the genus level using Mothur. In case a sequence could not be classified to the genus level, the closest level of classification to the genus level was given, preceded by "unclassified_".

In addition to collecting caecotrophs, food consumption was measured daily after weaning and careful visualization of appearance of ERE compatible signs (Table R2) was performed in order to detect those rabbits that develop ERE. 
Table R2. Gross lesions, symptoms and signs detected in rabbits that developed ERE.

\begin{tabular}{|c|c|c|c|c|c|c|}
\hline $\begin{array}{c}\text { Rabbit } \\
\text { ID }^{\mathrm{a}}\end{array}$ & $\begin{array}{c}\text { Prostratio } \\
\mathrm{n}\end{array}$ & $\begin{array}{c}\text { Diarrhe } \\
\mathrm{a}\end{array}$ & $\begin{array}{l}\text { Mucus } \\
\text { in } \\
\text { colon }\end{array}$ & $\begin{array}{c}\text { Stomach/small } \\
\text { intestine filled } \\
\text { with } \\
\text { gas/liquid }\end{array}$ & $\begin{array}{c}\text { cecal } \\
\text { Impaction }\end{array}$ & $\begin{array}{l}\text { Cecum filled } \\
\text { with } \\
\text { gas/liquid }\end{array}$ \\
\hline $\mathrm{R} 4$ & $\mathrm{P}^{\mathrm{b}}$ & $\mathrm{P}$ & & $\mathrm{P}$ & $\mathrm{P}$ & $\mathrm{P}$ \\
\hline $\mathrm{R} 17$ & $\mathrm{P}$ & $\mathrm{P}$ & & $\mathrm{P}$ & $\mathrm{P}$ & \\
\hline R11 & $\mathrm{P}$ & $\mathrm{P}$ & $\mathrm{P}$ & $\mathrm{P}$ & & \\
\hline $\mathrm{R} 15$ & $\mathrm{P}$ & $\mathrm{P}$ & $\mathrm{P}$ & $\mathrm{P}$ & & \\
\hline R16 & $\mathrm{P}$ & $\mathrm{P}$ & & $\mathrm{P}$ & & $\mathrm{P}$ \\
\hline $\mathrm{R} 18$ & $\mathrm{P}$ & $\mathrm{P}$ & $\mathrm{P}$ & $\mathrm{P}$ & $\mathrm{P}$ & $\mathrm{P}$ \\
\hline R8 & $\mathrm{P}$ & $\mathrm{P}$ & & $\mathrm{P}$ & $P$ & \\
\hline R24 & $\mathrm{P}$ & $\mathrm{P}$ & $\mathrm{P}$ & $\mathrm{P}$ & $\mathrm{P}$ & $\mathrm{P}$ \\
\hline $\mathrm{R} 25$ & $\mathrm{P}$ & & $\mathrm{P}$ & $\mathrm{P}$ & & $\mathrm{P}$ \\
\hline R27 & $\mathrm{P}$ & $\mathrm{P}$ & $\mathrm{P}$ & $\mathrm{P}$ & & $\mathrm{P}$ \\
\hline R31 & $\mathrm{P}$ & $\mathrm{P}$ & & $\mathrm{P}$ & $\mathrm{P}$ & $\mathrm{P}$ \\
\hline
\end{tabular}

${ }^{a}$ Rabbits are sorted in the same order as ERE rabbits shown in Figure R34. Only those rabbits that developed ERE are indicated in the table. Healthy rabbits did not develop any of the symptoms, signs and lesions shown in the table.

${ }^{\mathrm{b}} \mathrm{P}=$ presence of a particular lesion, symptom or sign.

While most rabbits (62\%) increased their food intake during the 3 weeks period and did not develop any ERE compatible sign, a total of 11 rabbits developed a sharp decrease in food intake ( $>50 \%$ reduction) during days 7 to 12 after weaning (Figure R34). None of these 11 rabbits increased their food intake afterwards (Figure R34) and all rabbits developed clinical signs compatible with ERE including diarrhea and prostration (Table R2). A necropsy was performed to corroborate ERE development in these 11 rabbits. Gross lesions compatible with ERE were observed in all rabbits that developed a sharp decrease in food intake (Table R2). However, consistent with ERE, no 
macroscopic signs of inflammation or congestion were observed in the intestinal tract of none of these rabbits. As expected, no gross lesions compatible with ERE were observed in those rabbits that did not develop a sharp decrease in food intake, neither signs of disease. In summary, $38 \%$ of the rabbits under study spontaneously developed ERE.

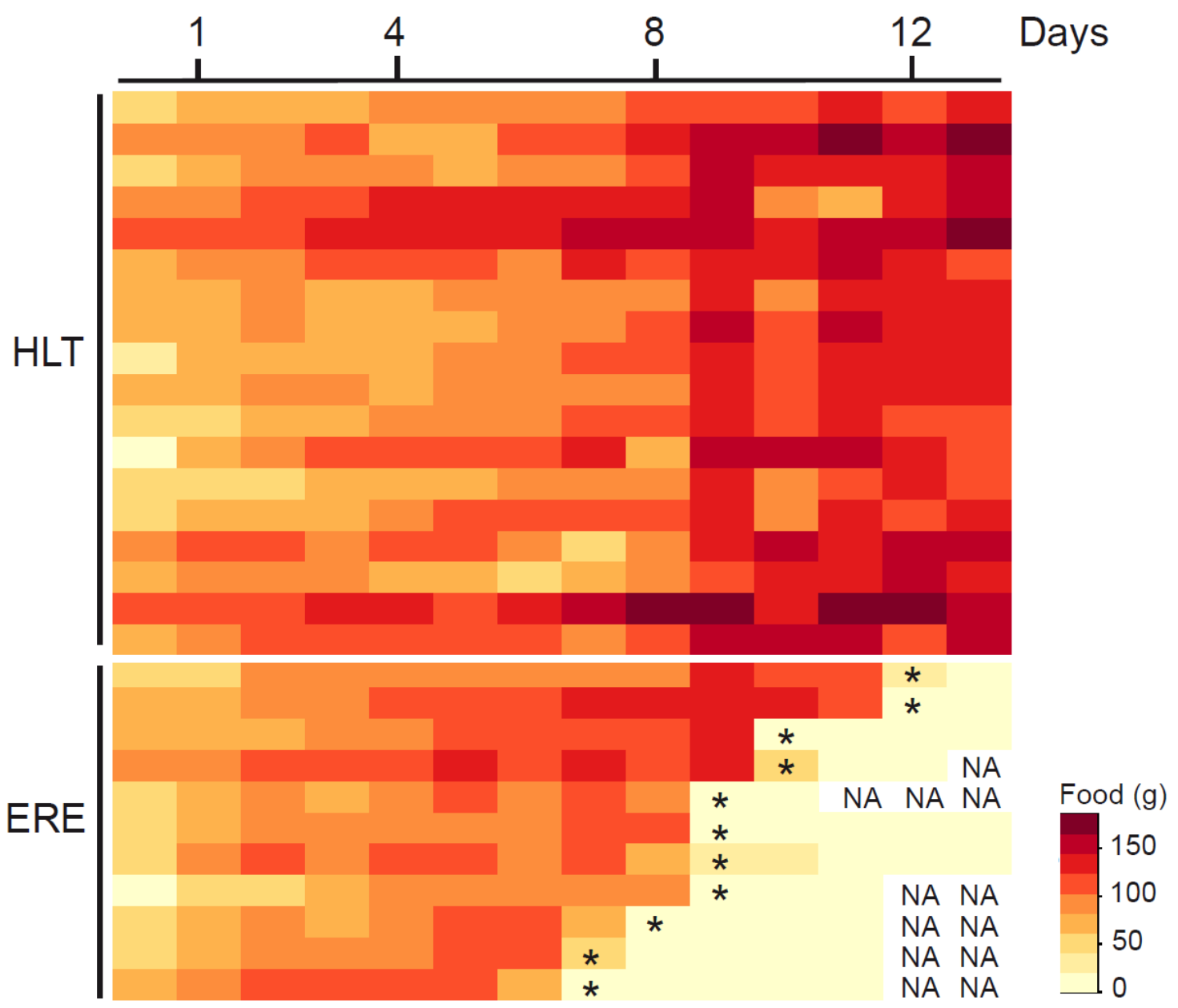

Figure R34. Food intake by rabbits after weaning: the amount of food (g) consumed by rabbits at different days after weaning is shown as a color-coded heatmap. Day $0=$ Day of weaning. NA: not analyzed due to death or euthanasia of that particular rabbit. The day of ERE onset as revealed by a sharp drop in food intake ( $>50 \%$ reduction compared to the previous day) is indicated with an asterisk. Healthy rabbits were followed for another week. No decrease in food consumption was observed during this additional week neither signs of disease in healthy rabbits.

As previously mentioned, microbiota dysbiosis has been associated with ERE (89). Nevertheless, the study published by Bauerl et al. (89) is the only one that describes the microbiota dysbiosis in ERE rabbits using high-throughput sequencing techniques. In 
order to confirm these results in rabbits from this study, the microbiota composition of samples collected after ERE onset was studied. The ERE onset was defined as the day in which a sharp drop in food consumption was detected (see Methods and Figure R34). For identifying bacteria that characterize ERE, 16S rRNA high-throughput sequencing using the 454 Roche platform was performed. The overall microbial diversity was analyzed by calculating the Shannon index, which takes into account the number and relative abundance of OTUs found in a given sample. OTUs analysis showed that the microbiota from rabbits with ERE was less diverse than the one from healthy controls (lower Shannon index, $\mathrm{P}<0.05$, Figure R35A). Subsequently, the overall structure of the microbiota of healthy and ERE rabbits was analyzed in order to detect potential differences. The microbiota similarity was quantified by applying the Yue \& Clayton measure of dissimilarity that compares the relative abundance of OTUs present in 2 given pair of samples. PCoA was used to cluster samples along orthogonal axes of maximal variance. The first principal coordinate of analysis (PC1) separated samples by health status indicating that the microbiota structure from rabbits with ERE differ from that of healthy controls (Figure F35B). Analysis of the molecular variance of the Yue \& Clayton dissimilarity values confirmed that this difference was statistically significant (AMOVA, $\mathrm{P}<0.001$ ). The non-parametric Wilcoxon test was performed in order to identify specific microbial changes in ERE rabbits. To avoid false positives, the FDR was applied. At the genus level, the relative abundance of 12 bacterial genera were significantly different $(\mathrm{FDR}<0.05)$ between rabbits with ERE and healthy controls (Figure F35C). Specifically, the microbiota of rabbits with ERE had higher levels of the genera Clostridium, Turicibacter, Robinsoniella, Coprobacillus, Roseburia, Bacteroides, Alistipes, Akkermansia, Campylobacter and Anaerovax and lower levels of Fusibacter and Adlercreutzia. When using a less stringent FDR threshold (FDR $<0.1)$, the abundance of 21 out of 32 analyzed genera differed between rabbits with ERE and healthy controls. On the other hand, the relative abundance of 11 OTUs $(F D R<0.05)$ and 34 OTUs $(\mathrm{FDR}<0.1)$ was found to be significantly different between both groups. Altogether these data indicate that ERE, also in rabbits from this study, is associated with a marked intestinal microbial dysbiosis. 

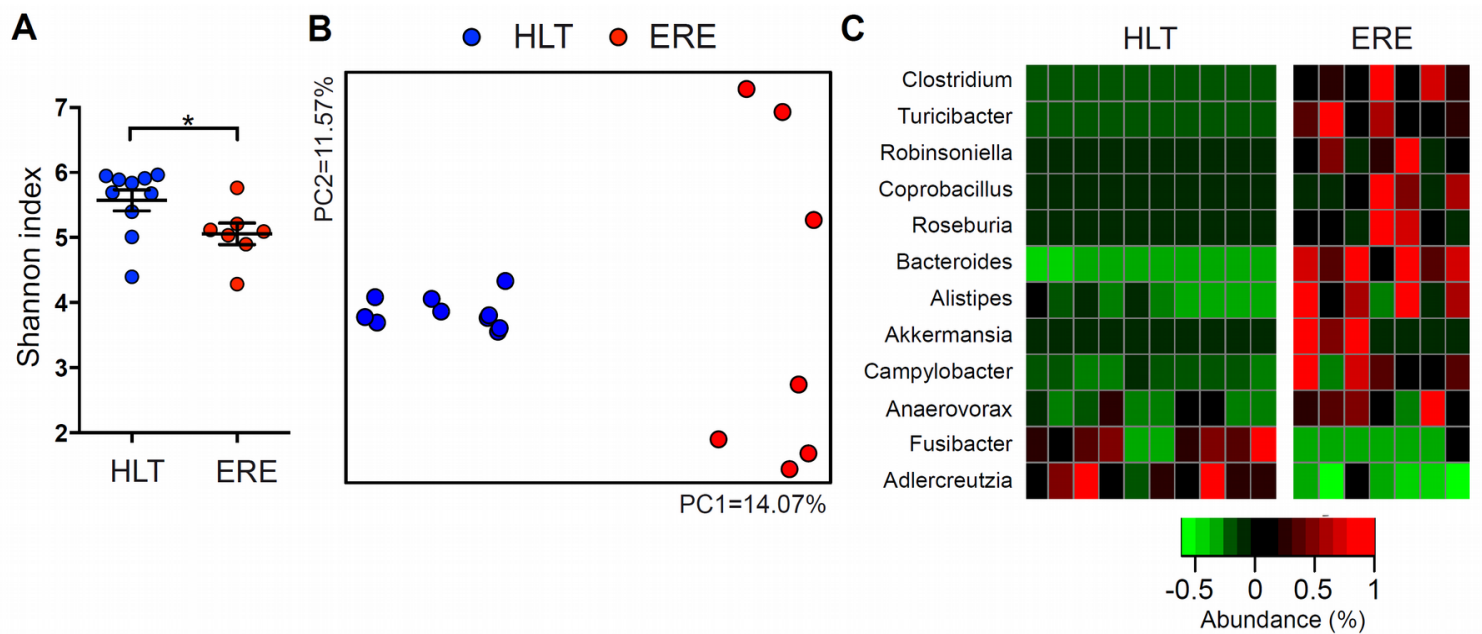

Figure R35. Epizootic rabbit enteropathy is characterized by dysbiosis of the intestinal microbiota. Microbiota composition was analyzed in caecotrophs samples from rabbits with ERE ( 1 to 3 days after ERE initiation) and compared with a similar time point (day 11 post-weaning) of healthy (HLT) littermate controls. (A) The Shannon index of the identified OTUs for each sample and mean +/- SEM for each group was calculated and plotted ( ${ }^{*} \mathrm{P}<0.05$, Wilcoxon test). (B) PCoA from the Yue \& Clayton values calculated between all the samples. (C) Heatmap representing the standard deviation from the mean of the relative abundance of statistically significantly different genera between rabbits with ERE and HLT littermate controls (Wilcoxon test, FDR $<0.05$ ). Red colors represent values above the mean and green colors represent values below the mean. $\mathrm{N}=10$ for the HLT group and 7 for the ERE group.

\subsubsection{Microbiota dynamics after the weaning and before the ERE onset in healthy and ERE rabbits}

In order to elucidate if the observed changes were consequence of the disease state or could be responsible for triggering ERE, the composition of the microbiota of rabbits before ERE onset was analyzed. The Yue \& Clayton index and PCoA were applied to analyze overall changes in the microbiota after weaning and before ERE onset. Changes of the microbiota over time could be identified in PC1 (Figure R36A). Interestingly, the day of weaning, samples from healthy rabbits are scattered distributed along the axis defined by PC1, suggesting variability of the microbiota at weaning. However, from day 7, samples move towards the positive values of the axis and cluster tightly at day 9 after weaning. From this day, samples keep clustering tightly between similar values of the PC1 axis. Importantly, samples obtained from mothers cluster tightly and are located within the same values as samples of days 9 to 21 after weaning. 
Remarkably, samples from ERE rabbits before disease onset (days 0 to 9 after weaning), move across the PCoA axis towards the positive values in a similar manner as healthy rabbit samples. This observation was supported by a linear regression analysis including interaction between disease state and time factors. Both groups fit a regression line with a similar slope over time given that the $\mathrm{P}$ value associated with the time factor was significant $(\mathrm{P}<0.0001)$ while no significance $(\mathrm{P}>0.05)$ was obtained for the disease factor or the interaction between time and disease. Moreover, when different time points were analyzed separately (Figure R36B), no separation was observed by disease state (AMOVA, $\mathrm{P}>0.05$ ). Altogether these results suggest that the microbiota structure of rabbits that will develop ERE, before ERE onset, is similar to that of healthy rabbits and evolve in a similar manner after weaning towards an adult microbiota state. 


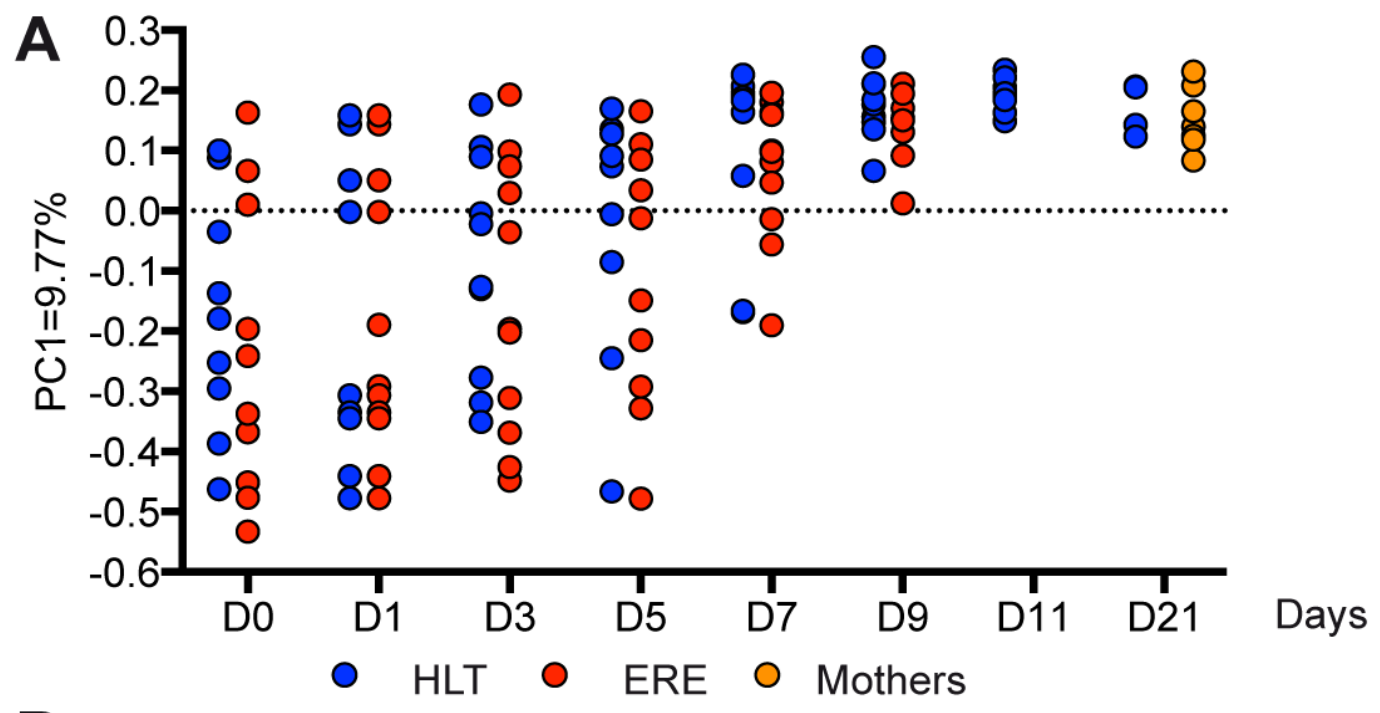

B
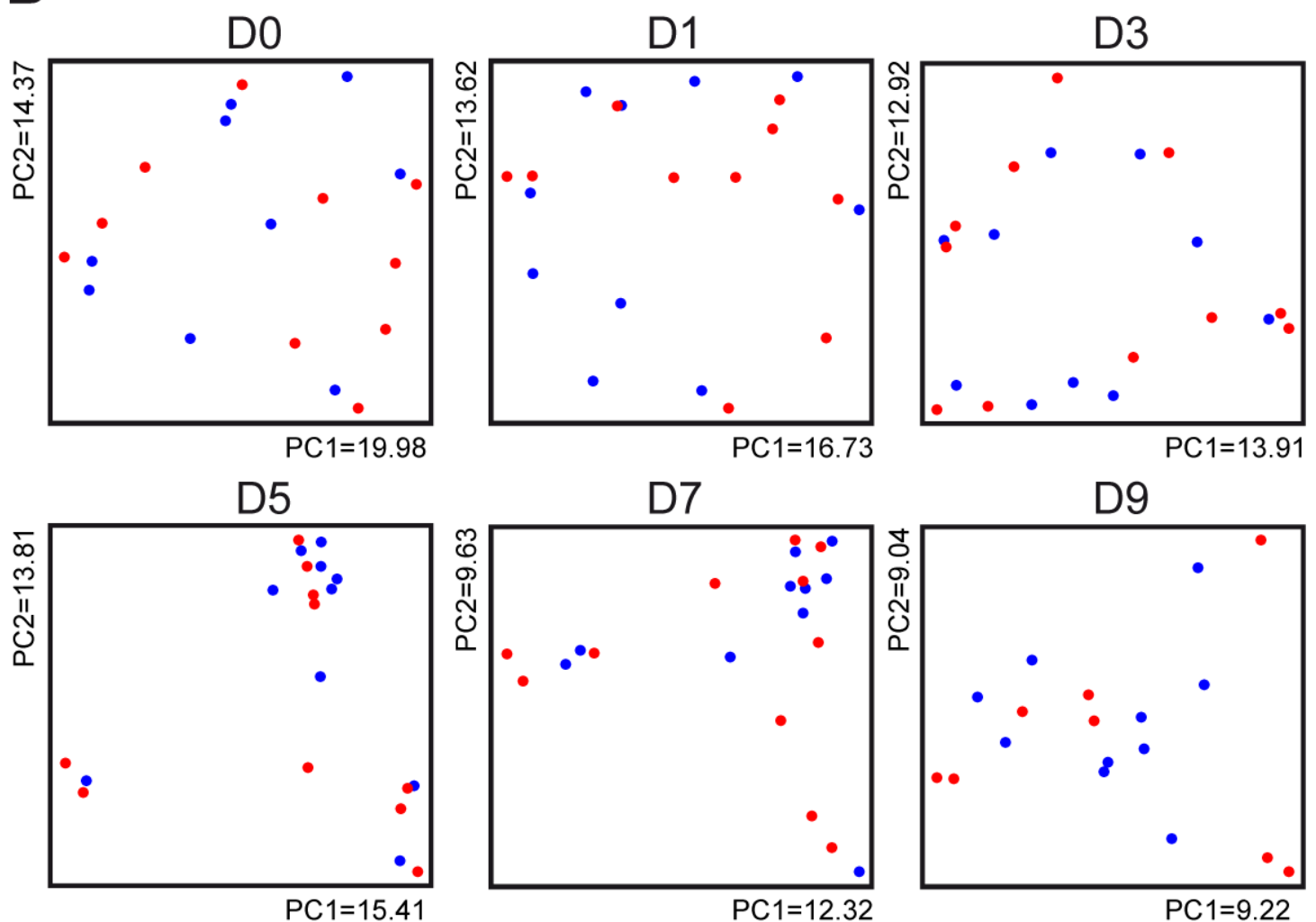

Figure R36. Evolution of the microbiota after weaning and before ERE onset. (A) First principal coordinate of analysis of the PCoA from the Yue \& Clayton values obtained between all samples plot over time. (B) PCoA at different days after weaning from the distance matrix generated using the Yue \& Clayton index between samples from a specific day. D0= day of weaning. Both in (A) and (B), samples from rabbits with ERE after disease initiation are not included in the analysis. Most of the rabbits ( 9 out of 11) initiate ERE before day 11 after weaning, thus samples from rabbits with ERE from this or subsequent days are not included. $\mathrm{N}=8-11$ per group except for day 21 where $\mathrm{N}=4$. Two consecutive samples from 4 
mothers, obtained the day of weaning and 7 days afterwards were also included in the analysis shown in (A).

Although the structure of the microbiota from healthy rabbits and those that will develop ERE does not differ before ERE onset, both groups could still differ in specific bacterial taxa. Thus, analysis were performed in order to confirm if any of the changes observed in rabbits with ERE (Figure R35C) could be detected before ERE onset (Figure R37). Remarkably, no significant differences in the abundance of any of the bacterial genera analyzed (Figure R37) were detected between healthy rabbits and those that will develop ERE, at any time point before ERE onset, even using a less stringent threshold of $\mathrm{FDR}<0.1$. On the other hand, the rest of bacterial genera that were not significantly different after disease onset, did also not differ between both groups before the beginning of the disease. Similarly, no differences between both groups were identified at the OTU level or other phylogenetic levels. Besides, no differences in the Shannon diversity index were observed before ERE onset. These results suggest that the dysbiosis observed in rabbits with ERE is a consequence of the disease rather than its initial cause. 

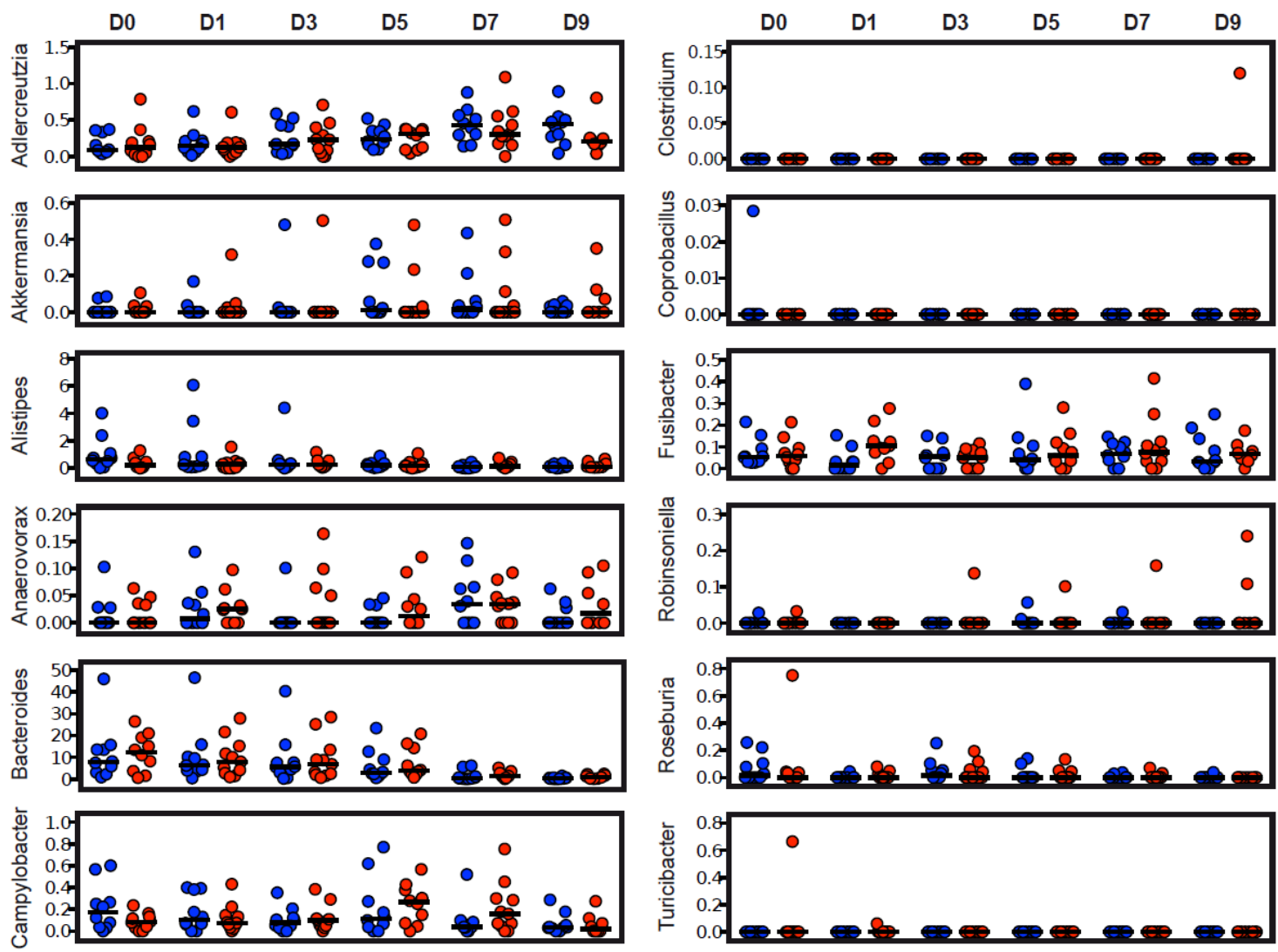

- HLT O ERE

Figure R37. Bacterial genera abundance in healthy and ERE rabbits before onset. Relative abundance at different days after weaning of bacterial genera that were found to be significantly different in rabbits with ERE (Figure R35C). Horizontal lines represent the mean for each group. D0 = day of weaning. Samples from rabbits with ERE after disease initiation are not included in the analysis. Most of the rabbits (9 out of 11) initiate ERE before day 11 after weaning, thus samples from rabbits with ERE from this or subsequent days are not included. $\mathrm{N}=8-11$ per group and time point.

\subsubsection{Comparison of the microbiota composition of ERE rabbits on the day of} disease onset with age-matched healthy littermate controls

Different rabbits start developing ERE at different days after weaning (Figure R34). If the observed microbiota dysbiosis was responsible for triggering the disease state, it could be possible that microbiota changes would have developed in different rabbits at different time points, resulting on different days of ERE onset. For this reason and in order to elucidate if the identified dysbiosis occurred exclusively after ERE onset, samples obtained the day of ERE onset were analyzed and compared to age matched healthy controls. For 8 out of 11 rabbits, a caecotroph sample was obtained the day of 
onset. Yue \& Clayton measure of dissimilarity and PCoA were used to compare the microbiota structure of these samples with that one from samples obtained after ERE onset and from samples of healthy controls collected at similar days after weaning. The microbiota from samples obtained after onset differ from that one of samples collected the day of onset and from samples of healthy controls (AMOVA, $\mathrm{P}<0.001$; Figure R38A). However, no significant difference in the microbiota structure was observed between samples collected the day of onset with those from age-matched healthy controls (AMOVA, P $>0.05$; Figure R38A). Similar results were obtained using UPGMA hierarchical clustering (Figure R38B). Moreover, no statistically significant differences were detected between healthy controls and samples collected the day of ERE onset in any bacterial genera whose abundance was found to be altered after ERE onset (Figure R38C), neither in any other bacterial taxa or OTU (see Methods for the different comparisons performed using different healthy control groups). Besides, no differences in the Shannon diversity index were observed between samples from ERE rabbits the day of onset and age-matched healthy littermate controls. Altogether these results confirm that the dysbiosis observed in the intestinal microbiota of rabbits with ERE is a consequence rather than the initial cause of the disease. 
A $\bullet$ HLT $\bigcirc$ ERE onset $\odot$ ERE after B
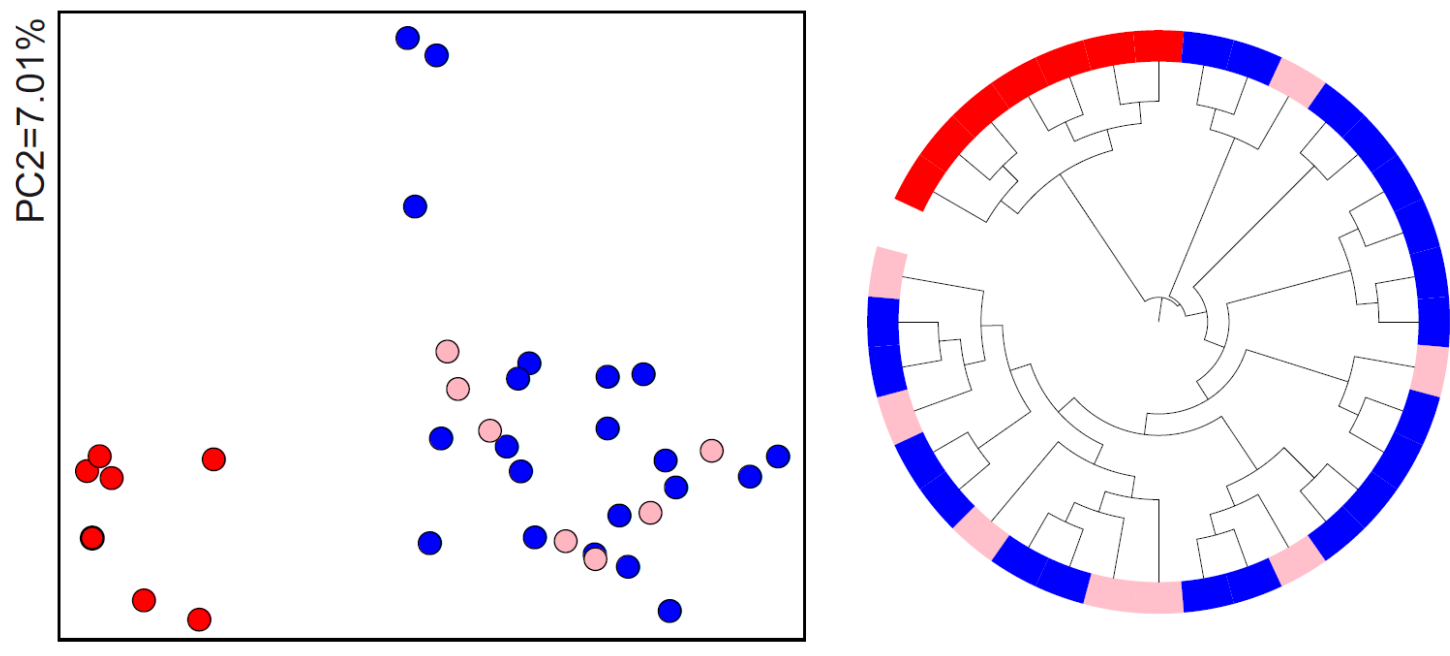

C

$\mathrm{PC} 1=8.51 \%$
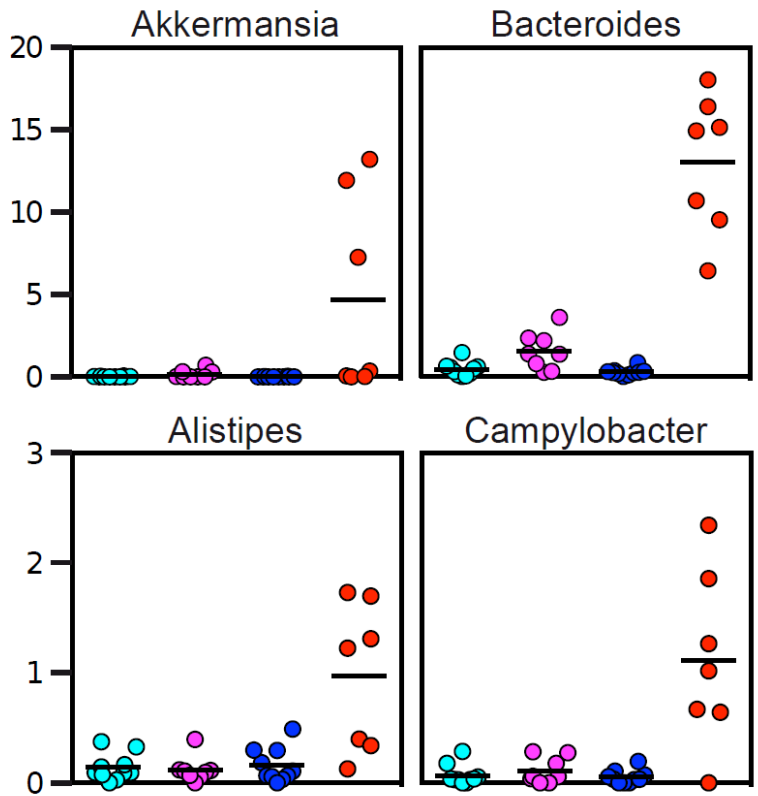

Campylobacter

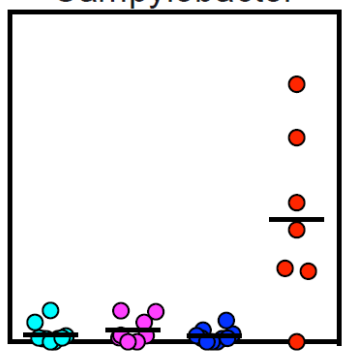

Coprobacillus
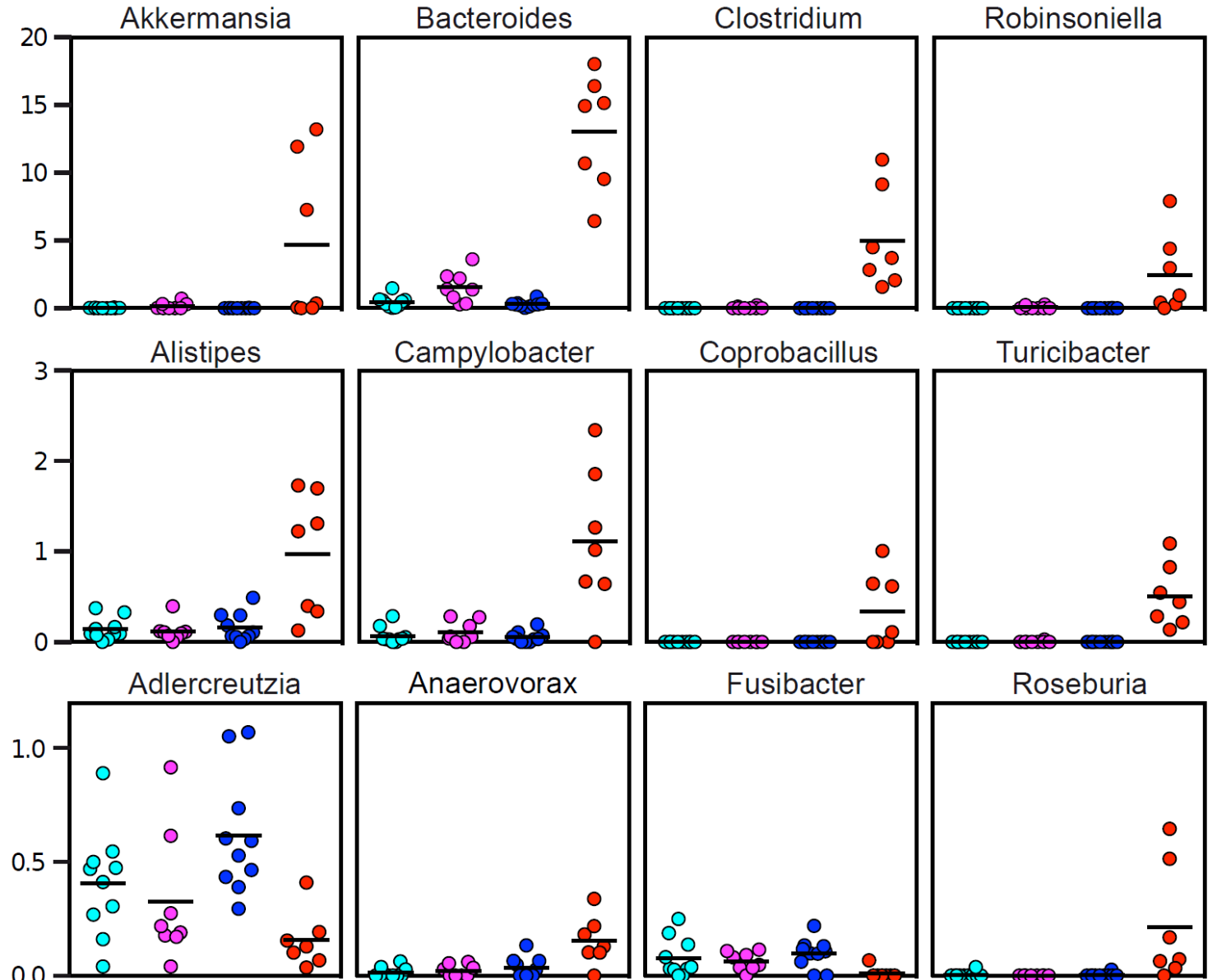

Anaerovorax
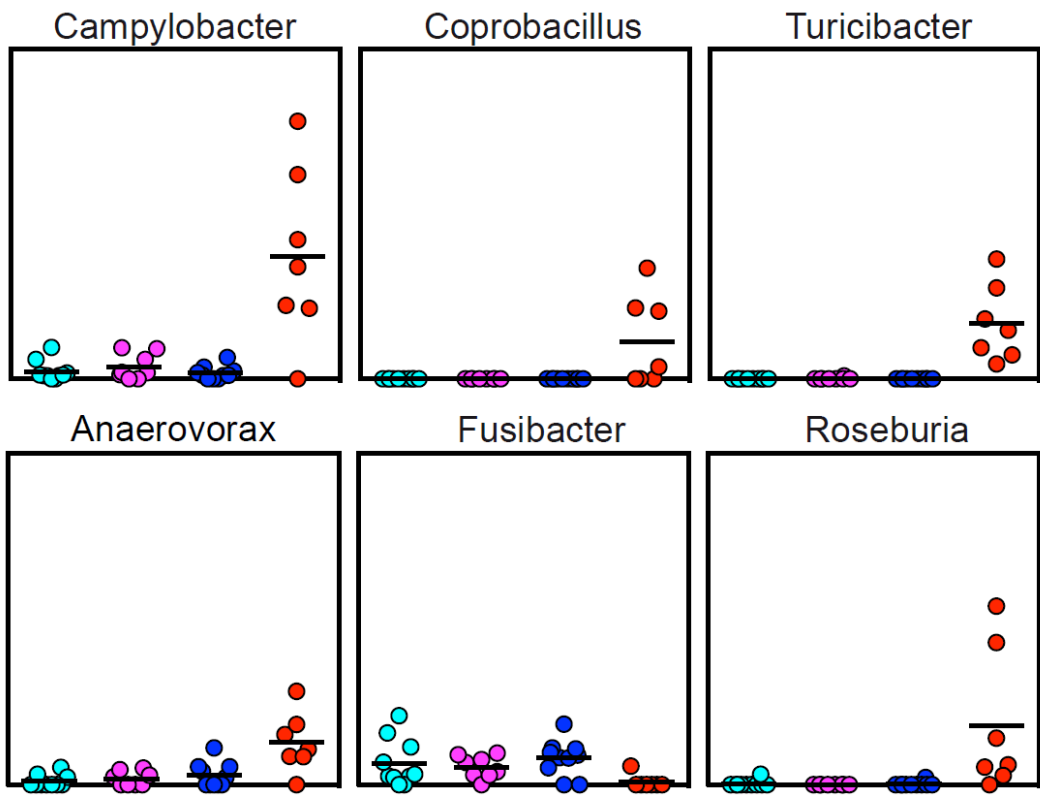

$$
\text { O HLTD9 }
$$

ERE onset

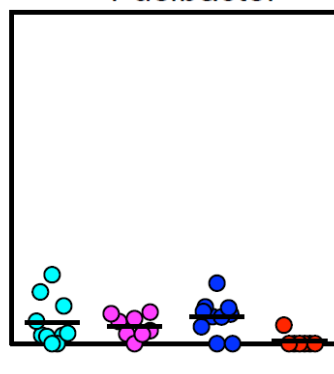

Roseburia

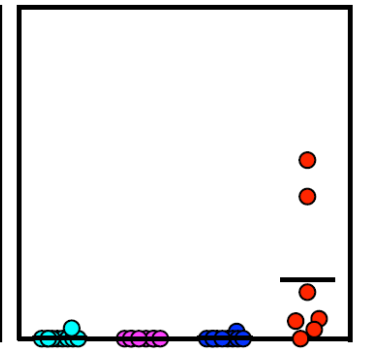

Figure R38. Microbiota dysbiosis occurs after ERE onset. (A) PCoA or (B) UPGMA hierarchical tree obtained from the Yue \& Clayton values calculated between samples from rabbits with ERE collected the day of onset or after onset and samples from healthy rabbits at similar time points (Days 9 and 11 after 
weaning). (C) Relative abundance of bacterial genera that were found to be significantly different in rabbits with ERE (Figure R35C) from samples obtained from sick rabbits the day of onset or after onset, compare with samples from healthy littermate controls at similar time points. $\mathrm{N}=7-10$ per group and time point. The mean for each group is shown.

\subsubsection{Higher sequencing coverage using Illumina platform identifies a unique microbiota change the day of ERE onset}

Although previous results did not identify any change that precedes ERE development, there was still the possibility that changes in low abundant bacteria between healthy and ERE rabbits before the disease onset have not been identified due to the low number of sequences $(2849 \pm 779)$ obtained by 454 sequencing technology. For this reason, all samples were re-sequenced using the newer Illumina platform (not available at the beginning of the study), which now allows obtaining higher sequencing coverage per sample at a similar cost. Analysis performed on sequences obtained using this new platform revealed results similar to those represented in Figure R35B when comparing the microbiota composition in samples obtained after ERE onset to that of healthy littermates (AMOVA, $\mathrm{p}<0.001$ ). Besides, the microbial diversity represented by Shannon index was lower in rabbits that developed ERE compared to healthy animals $(\mathrm{P}<0.05)$. In order to detect specific changes in the microbiota composition after ERE onset, non-parametric Wilcoxon test with FDR was applied. At the genus level, the relative abundance of 24 bacterial genera were significantly different $($ FDR $<0.05)$ between rabbits with ERE and healthy controls. Importantly, analysis performed on sequences obtained by Illumina confirmed higher abundances of the genera Clostridium, Turicibacter, Robinsoniella, Bacteroides, Akkermansia, Campylobacter and Anaerovorax in those rabbits that developed ERE, which was already identified with the 454 platform (Figure R35C). Lower levels of the genus Adlercreutzia in ERE rabbits were also detected using the Illumina platform. Besides, the relative abundance of 7 OTUs $(\mathrm{FDR}<0.1)$ was found to be significantly different between the groups of healthy and ERE rabbits.

In order to confirm that there were no changes in the microbiota composition before the disease onset in ERE rabbits, samples obtained after the weaning from the 2 
groups of rabbits were compared. No statistically significant differences (FDR $<0.1$ ) were observed at the genus or OTU level at any day post-weaning and before ERE onset.

Since not all rabbits develop ERE on the same day post-weaning, as previously described, comparison of samples from ERE rabbits obtained on the day of the disease onset and samples obtained from healthy rabbits on day 9 post-weaning was performed. Interestingly, analysis revealed significantly higher abundance $(\mathrm{FDR}<0.1)$ of the genus Clostridium in ERE rabbits (Figure R39A). Moreover, this difference was basically due to the presence of a single Clostridium OTU (OTU31) in ERE rabbits, which was absent in the healthy animals (Figure R39B). This result suggests that the OTU31 may have a key role in the initiation of ERE.

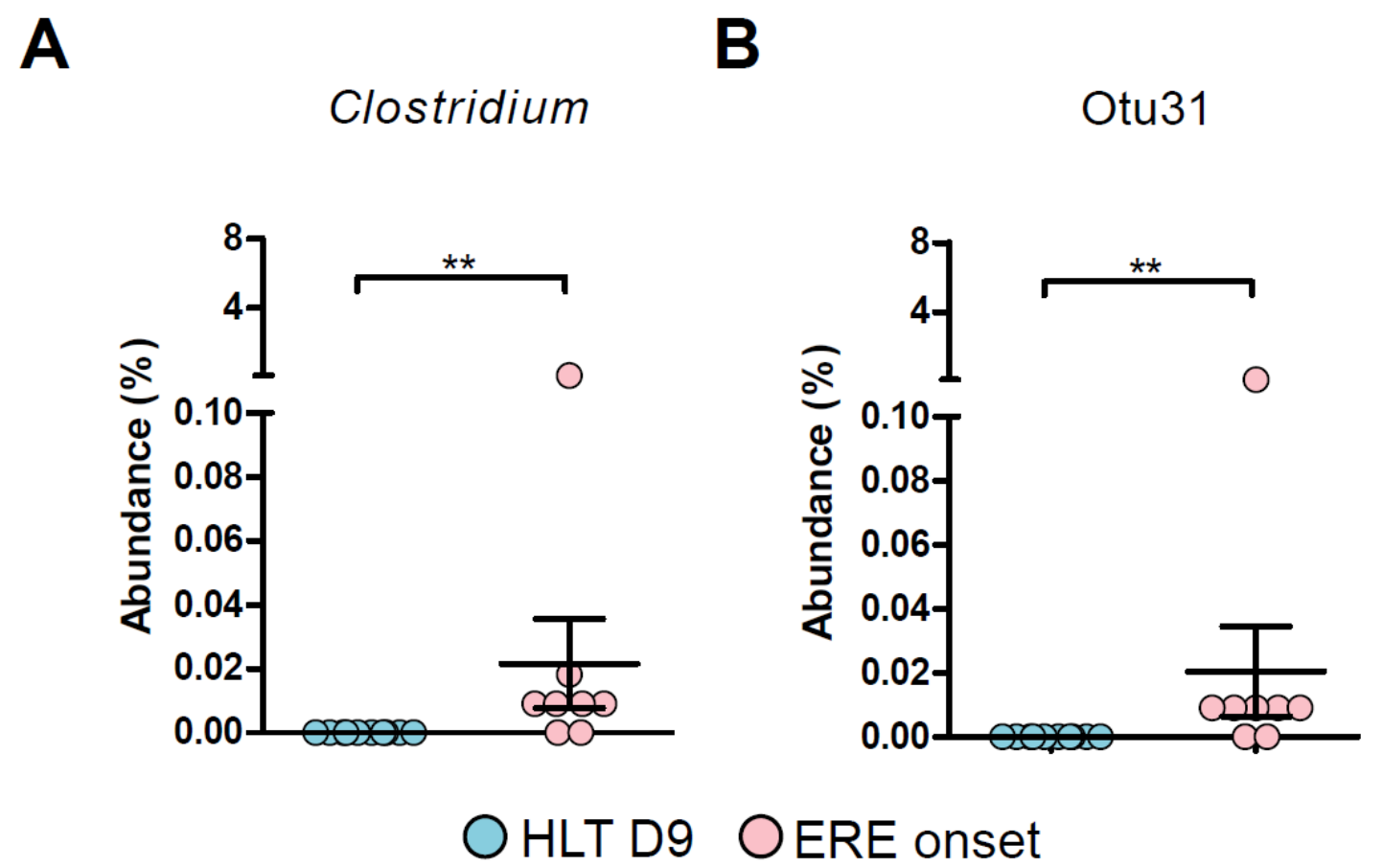

Figure R39. The relative abundances of taxa differently abundant between samples of healthy rabbits collected on day 9 post weaning (HLT_D9) and samples from ERE rabbits on the day of onset (ERE_onset). (A) Clostridium is present in higher abundance $(* * p<0.01$; FDR $<0.1)$ in rabbits that develop ERE, and this difference in relative abundance is due to only 1 single OTU $(* * p<0.01)$ (B). Means and + - SEM values are represented.

To confirm the association of the Clostridium OTU31 with the development of ERE, all sequences obtained by Illumina from all healthy rabbits' samples (1 392113 sequences, 74 samples in total) were aligned against the representative sequence of 
OTU31. Blast analysis revealed that none of 1392113 sequences correspond to the OTU31 (none of the sequences shown more than $95 \%$ of similarity to Clostridium OTU31). In contrast, OTU31 was found to be present in samples collected before the disease onset in all but 1 ERE rabbit, in which indeed it was possible to detect this OTU after the disease onset. Altogether, these results suggest that this OTU is characteristic of ERE rabbits and may be involved in the initiation of the disease.

\subsubsection{The effect of decreasing food consumption on ERE development and microbiota dysbiosis}

As previously described, a drastic decrease in food consumption is considered the first sign of ERE, which occurs in all rabbits that develop the disease. Although unlikely, it could be possible that food restriction could be the trigger that promotes dysbiosis and ERE development. For this reason an experiment was performed in which food consumption was restricted in a group of rabbits, as described in Methods. Since the majority of the rabbits develop ERE between the first and second week after weaning, food deprivation was performed in 2 groups of rabbits, initiating the food restriction on the first or the second week post-weaning. In order to assess the effect of exclusively food restriction on the microbiota composition, all rabbits that developed ERE were excluded from analysis, since ERE rabbits have marked microbiota dysbiosis and this could mask the changes in the microbiota produced as a result of food restriction. Importantly, there was no difference in the number of rabbits that developed ERE between the group of rabbits subjected to food restriction and the control group, suggesting that food restriction does not promote ERE. Subsequently, microbiota analysis was performed from cecal contents collected from the control group of rabbits with unlimited access to food and from the group of rabbits in which food was restricted. As a control, the cecal content from another group of rabbits with unlimited access to food and which have developed ERE was also analyzed. Yue \& Clayton measure of dissimilarity and PCoA were used to compare the microbiota structure from these 3 groups of rabbits. As shown in Figure R40, the microbiota from samples obtained after ERE onset differ from those samples collected after 2 days of food restriction and from samples of healthy controls (AMOVA, $\mathrm{P}<0.001$ ), independently whether restriction was 
performed at 1 or 2 weeks post-weaning (Figure R40A and Figure R40B, respectively). Interestingly, the microbiota from samples obtained after 2 days of food restriction, independently whether restriction was performed at 1 or 2 weeks post-weaning, was significantly different from the microbiota of healthy rabbits with unlimited access to food (AMOVA, $\mathrm{P}=0.002$; Figure R40), suggesting that the food deprivation produced changes in the microbiota. Altogether, these results suggest that the overall changes observed after ERE onset could not be explained by reduction in food consumption, although reduction in food consumption does change the microbiota.

A

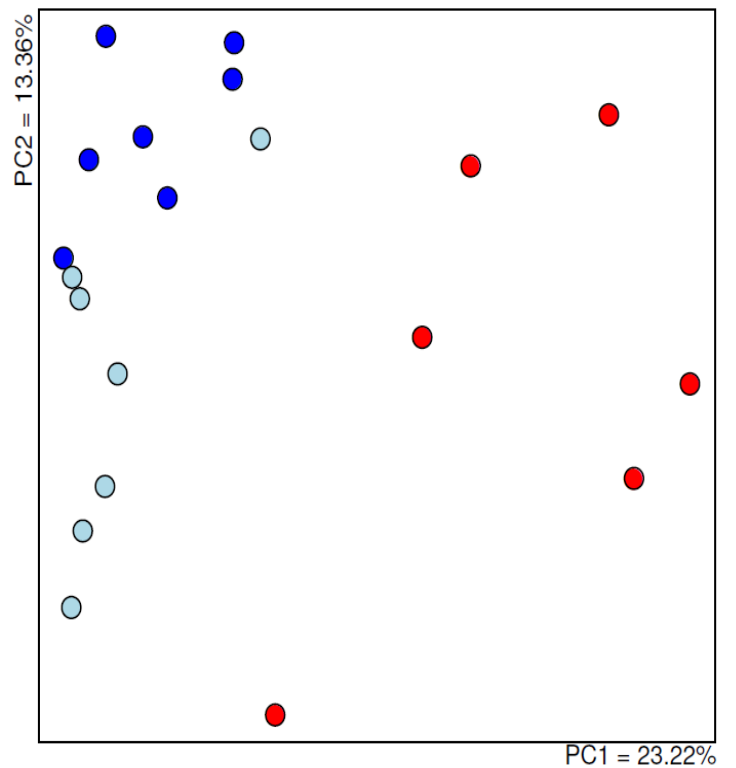

B

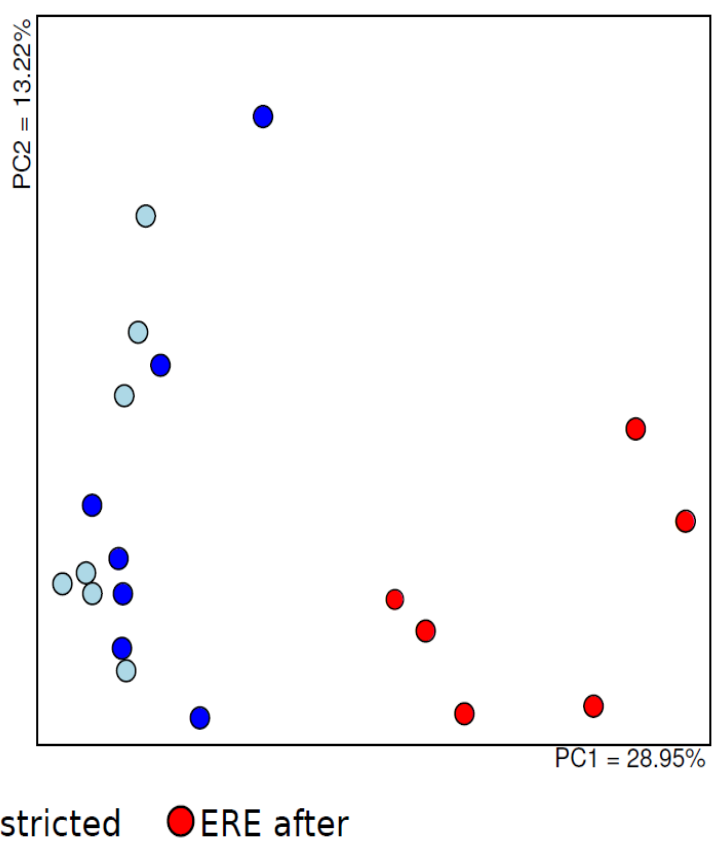

Figure R40. Food restriction does not reproduce the microbiota dysbiosis observed after ERE onset. The overall microbial composition in samples from healthy rabbits subjected to food deprivation for 2 consecutive days (HLT restricted), starting 7 days after weaning (A) or 14 days post-weaning (B). As control, samples collected from age-matched healthy control groups (HLT control) and from rabbits that develop ERE (ERE after), were also analyzed.

Subsequently, the non-parametric Wilcoxon test with FDR was applied in order to identify specific changes in the microbiota induced by food restriction. When compared to control, samples obtained after food restriction performed on the second week post-weaning revealed a higher relative abundances $(\mathrm{p}<0.01 ; \mathrm{FDR}<0.1)$ of the 
genera Anaerovorax and Olsenella, and lower abundance $(\mathrm{p}<0.01 ; \mathrm{FDR}<0.1)$ of Anaeroplasma. Similarly, Anaerovorax was detected as a genus with significantly higher abundance $(p<0.01)$ in the group of food restriction on the first week post-weaning. These results suggest that the higher abundance of Anaerovorax in ERE rabbits could be explained, by the decrease in the food consumption (Figure R41).

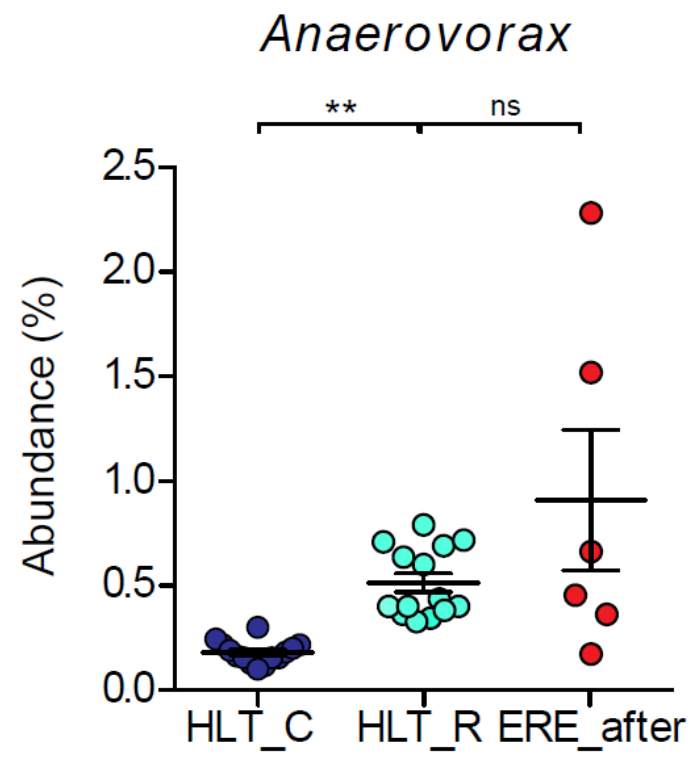

Figure R41. Higher abundance of the genus Anaerovorax in ERE rabbits could be due to the decrease in the food consumption in this group of rabbits. Relative abundance of the genus Anaerovorax in samples collected from healthy control group (HLT_C), samples collected after 2 days of food deprivation (HLT_R) and samples from ERE rabbits.

\subsubsection{Analysis of the genome of the OTU31 classified as Clostridium and associated with ERE onset}

Having discarded the role of food restriction in ERE development and major dysbiosis, the investigation was focused on the Clostridium OTU31, identified as a possible etiological agent of ERE. Indeed, several species of Clostridium (e.g. C. botulinum, C. tetani, C. difficile) are known etiological agents of infectious diseases both in humans and animals. Moreover, for over a decade, it has been suggested that Clostridium spp. could be a possible etiological agent of ERE (94).

In order to study in more detail the Clostridium OTU31 and determine its potential virulence capabilities and role in ERE, an attempt to isolate this OTU from 
fecal samples of ERE rabbits was performed. After analysis of more that 1,000 colonies, we were able to isolate 1 bacterial colony whose 16S RNA gene sequence had $100 \%$ similarity to the representative sequence of OTU31. Subsequently, the genome of this strain was sequenced as described in Methods. Based on phylogenomic analysis of 92 genomes of members of the Clostridiales order, the OTU identified as a putative etiological agent of ERE was demonstrated to be a novel species of the genus Clostridium, which we named Clostridium cuniculi (Figure R42). Moreover, the closest neighbors of $C$. cuniculi are $C$. botulinum and $C$. perfringens, 2 well-known pathogens. Interestingly, both $C$. botulinum and $C$. perfringens produce potent toxins, which induce muscle paralysis, a major cause of the disease symptoms observed during the course of the disease induced by these pathogens. Interestingly, as mentioned in Introduction section 1.2.3.3, intestinal paralysis has been suggested to be the cause of some of the signs observed in ERE (e.g. cecum impaction) (92). For these reasons, all genes that could encode for toxins or other putative virulence factors were identified in the genome of the OTU31. 


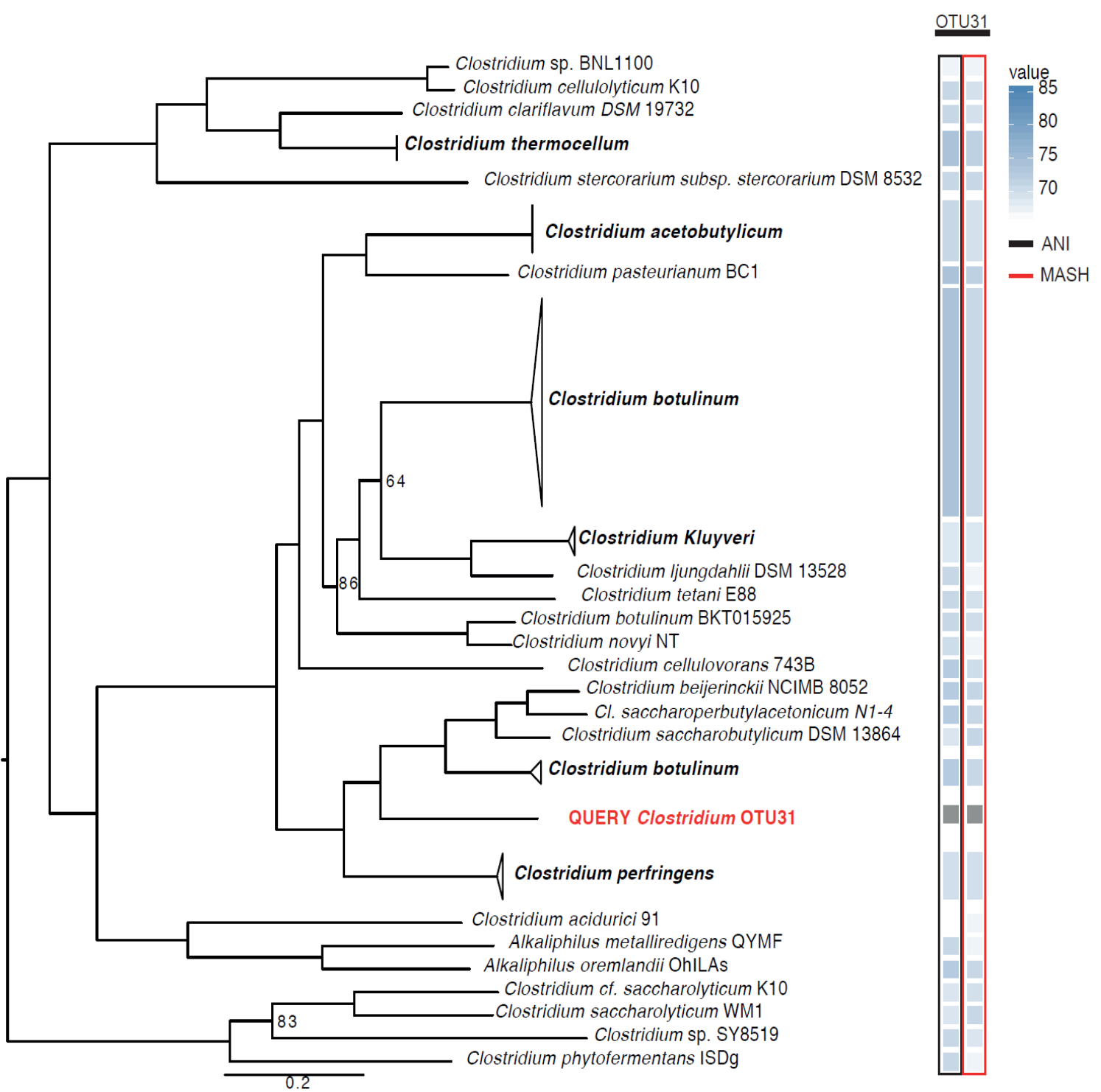

Figure R42. The OTU31 is a new species of the genus Clostridium. The tree represents the best phylogenetic location of the OTU31 and species belonging to the genus Clostridium. The bootstrap value is stated when it was lower than $95 \%$. The heatmap represents percentage of identity of the OTU31 with other species of the genus Clositridum calculated by implementation of ANI and MASH (see Methods).

The analysis of the genome revealed the presence of 2 virulence factors: exoU, a type III effector protein with cytotoxic properties, and a gene encoding for a hemolysin. Moreover, an operon strikingly similar to the operon present in C. botulinum, which encodes the type A neurotoxin complex, was also identified (Figure R43). This complex in $C$. botulinum is composed by the botulinum neurotoxin (BoNT), several toxin 
associated proteins known as the hemagglutinins (HAs), the transcription factor botR, and the non-toxic non-hemagglutinin protein (NTNH) (131). BoNT functions as Zincdependent metalloprotease that inhibits the release of neurotransmitter acetylcholine from peripheral cholinergic synapses, resulting in muscle relaxation. As shown in Figure $\mathbf{R 4 3}$, the operon detected in the genome of C. cuniculi contains all the HAs genes and a transcriptional factor similar to that one present in an operon encoded by $C$. botulinum. In contrast, $C$. cuniculi is lacking the gene that encodes BoNT, and instead encodes a protein with domains similar to those present in hemagglutinins (ricin domain) and in serine protease inhibitors. Interestingly, the inhibition of a serine protease (acetylcholinesterase) is known to cause paralysis (132), an event that is thought to be the cause of some of the signs observed in ERE.

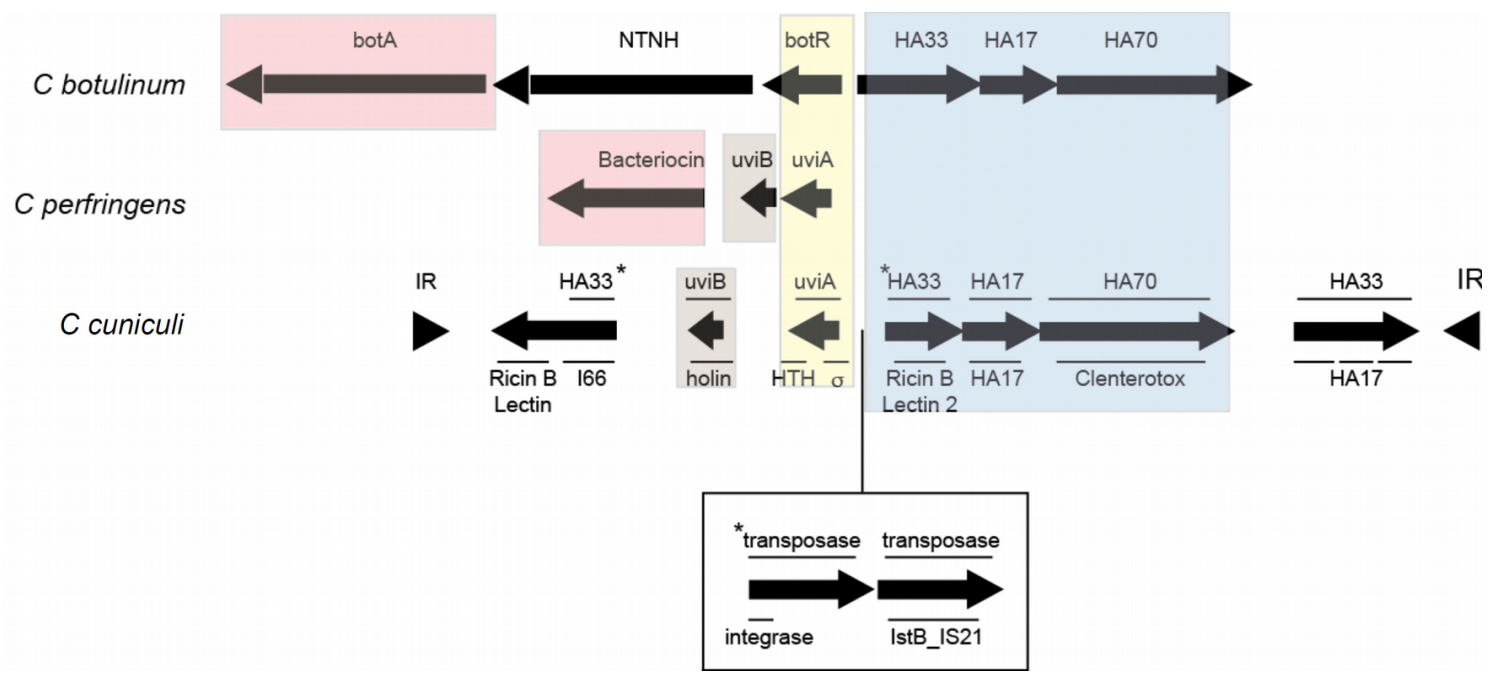

Figure R43. The operon detected in the genome of $\boldsymbol{C}$. cuniculi and corresponding operons in genomes of $\boldsymbol{C}$. botulinum and $\boldsymbol{C}$. perfringens. The names above genes encoded by the operon detected in the genome of $C$. cuniculi correspond to the best match obtained by aligning the sequence of the proteins encoded by the genes of the operon against the NCBI database. The asterisk marks genes that are only partially similar to the indicated genes. The names bellow genes encoded by the operon correspond to the protein domains identified in amino-acid sequences encoded by these genes using InterPro and considering the most significant hit. In yellow are marked transcription regulators, in red effector proteins, in blue hemagglutinins, and in brown holins. BotA- botulinum toxin A; NTNH- nontoxic, nonhemagglutinin protein; HA- hemagglutinins; IR- inverse repeats. 


\subsubsection{Attempt to reproduce ERE by administration of the $C$. cuniculi to healthy SPF rabbits}

In order to test the role of $C$. cuniculi in ERE development, an attempt to reproduce the disease was performed, as described in Methods. Since some authors were able to reproduce ERE in healthy rabbits by administration of the inoculum obtained from cecal content of animals that developed the disease $(93,94)$, a group of rabbits was orally inoculated with cecal content of animal that developed ERE as a positive control. The second group was orally inoculated with $10^{8} \mathrm{CFUs}$ of the C. cuniculi isolated strain, while the third group served as a negative control and was not inoculated with any bacteria. All 3 groups of rabbits were observed for appearance of ERE signs, and their food consumption was measured daily. No specific reduction in food consumption was observed in the groups of rabbits that received C. cuniculi strain. On the other hand, although a slight reduction in food consumption was observed in the group of rabbits that were inoculated with the cecal contents from ERE rabbits (Figure R44), no specific signs such as mucus, diarrhea, or cecal impaction could be observed in any of the analyzed groups. These results suggest that our experimental setup was not able to reproduce the disease even with the inoculum obtained from ERE rabbits and therefore further attempts should be performed in order to fully elucidate the role of $C$. cuniculi in the initiation of ERE. 


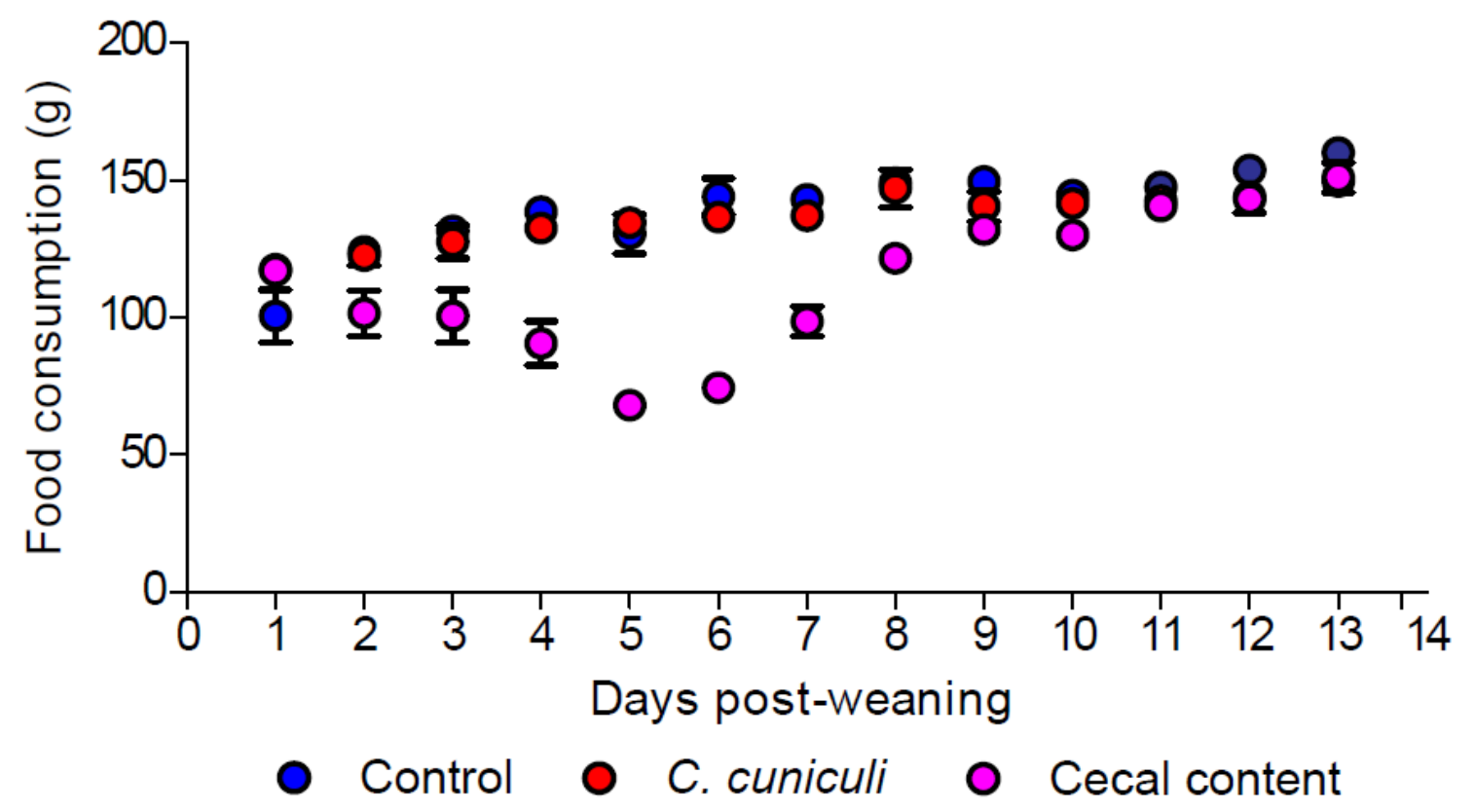

Figure R44. Food consumption changes during the attempt to experimentally reproduce ERE. Food consumption (in grams) measured during 2 weeks in a control group of rabbits that received PBS (Control), a group of rabbits to which C. cuniculi strain was administered, and a group of rabbits to which cecal contents of ERE rabbits was administered (cecal content). One-way ANOVA revealed significantly different means between the control group and the cecal content group $(\mathrm{p}<0.01)$, while no difference was observed between control and C. cuniculi group ( $\mathrm{N}=10$ rabbits per group). 




\section{Discussion}

\subsection{Microbiota dysbiosis and MRE colonization and persistence in mice}

The intestinal microbiota provides the protection against the colonization with intestinal pathogens, a phenomenon known as "colonization resistance" (133). On the other hand, microbiota dysbiosis as a result of antibiotic treatment is associated with the loss of colonization resistance and, consequently, intestinal tract colonization by antibiotic-resistant pathogens, such as MRE. In a study published by Hoyen et al. (87) authors demonstrated that not all antibiotic treatments affect equally the capability of $K$. pneumonieae to colonize the intestine of the mice, suggesting that some members of the intestinal microbiota participate in $\mathrm{CR}$, while others are dispensable. However, the effects of different antibiotic treatments on the microbiota composition and bacterial species that are necessary for the maintenance of the CR against MRE are not completely understood. In the first chapter of this thesis, we have investigated the effects of different antibiotic treatments on the microbiota, and subsequently, on MRE colonization and persistence using a mouse model. We specifically investigated the effect of 3 antibiotics (ampicillin, vancomycin and neomycin). Vancomycin is a glycopeptide antibiotic with activity mainly against Gram-positive bacteria, although a previous study that we performed in mice demonstrated that administration of this antibiotic could also result in alteration of Gram-negative bacteria (66). Consistent with the results from our previous study, mice treated with vancomycin had lower abundances of genera belonging to the phylum Bacteroidetes (Gram-negative) and Firmicutes (Gram-positive), while the phylum Proteobacteria underwent a drastic expansion. The depletion of the bacteria from the phylum Bacteroidetes is somehow paradoxical because this phylum contains bacterial species, which are resistant to vancomycin. Nevertheless, the resistance to vancomycin of Bacteroidetes species is frequently lower $(\mathrm{MIC} 50=4-128 \mu \mathrm{g} / \mathrm{ml})$ than the resistance observed in Proteobacteria species such as K. pneumonie (MIC50=128- 1024 $\mu \mathrm{g} / \mathrm{ml}$ ) (134-137). Taking into account the high concentrations that vancomycin can reach in the gut after oral administration $(500 \mu \mathrm{g} / \mathrm{g})(138)$, it is possible that while some of the Proteobacteria strains can survive in the gut after vancomycin treatment, most of the 
Bacteroidetes strains are depleted by the action of the antibiotic. Nevertheless, we cannot discard the possibility that some Firmicutes species that are depleted after vancomycin therapy could be important for the growth of Bacteroidetes species. Futures studies will be necessary to elucidate the mechanism by which basically all Bacteroidetes species are depleted after vancomycin treatment. On the other hand, mice were treated with ampicillin, a broad-spectrum antibiotic active against Gram-positive and some Gramnegative bacteria. In a study published by Ubeda et al. (65) ampicillin treatment resulted in the increase of the family Streptococcaceae in the mouse cecum, while the abundance of the family Erysipelotrichaceae and Lachnospiraceae decreased. In this study we confirmed that families Erysispelotrichaceae and Lachnospiraceae were decreased as a result of the ampicillin treatment. However, the increase in the abundance of the family Streptococcaceae could not be detected in mice treated with ampicillin as a part of this thesis, which could be explained by the fact that this family was not present in the fecal samples of most of the analyzed mice before treatment. In addition, our results indicate that mice treated with ampicillin had significantly higher levels of Proteobacteria, specifically the genus Parasutterella. On the other hand, neomycin is an antibiotic with a relatively narrow spectrum (mainly against Gram-negative bacilli but not against anaerobes, which account for most of the commensal bacteria identified in the gut). Thus, it was not surprising that the overall effect of neomycin on the microbiota composition was mild as compared to the effect of vancomycin or ampicillin. Overall, these results indicated that each of the tested treatments produced distinct changes in the microbiota composition.

Previous studies have shown that antibiotic treatment renders mice susceptible to colonization with antibiotic-resistant Enterobacteriaceae. In a recent study, Lewis et al. (139) demonstrated that the administration of vancomycin to mice results in high levels of intestinal colonization with carbapenem-resistant $K$. pneumoniae. In order to investigate the role of microbiota dysbiosis, as a result of ampicillin, vancomycin or neomycin treatments, on colonization with MRE, mice were infected with multidrug resistant Klebsiella pneumoniae (MRKP). We found that MRKP was unable to colonize the intestine of the untreated mice, as shown before by Donskey et al. (140). On the other hand, consistently with the different effect of the tested antibiotics on the microbiota 
composition, MRKP colonized at high levels the intestine of those mice treated with ampicillin or vancomycin, but not after neomycin treatment. Consistent with our results, other authors also reported the ability of $K$. pneumoniae to colonize the murine intestine after the ampicillin treatment (85). These results suggest that vancomycin and ampicillin treatment depleted bacteria that are necessary for the maintenance of the $\mathrm{CR}$, while neomycin treatment, although produced changes in the microbiota composition, did not affect the key bacteria necessary for the protection against MRKP intestinal colonization.

Importantly, we observed that once established, MRKP could persist in the intestine of the vancomycin and ampicillin treated mice for up to 1 month. These results were even more pronounced when the 3 antibiotics were given together. This last result is worrying since frequently, as described in the project 2 of this thesis, hospitalized patients received more than one antibiotic at the same time, which could render them more susceptible for colonization with the multidrug resistant pathogens. Moreover, our results are consistent with the ones obtained by Caballero et al. (85) in which the authors demonstrated the capability of MRKP to persist in the intestinal tract after ampicillin treatment. Nevertheless in their study (85), mice were infected concurrently with VRE and MRKP, thus it was possible that somehow VRE could be necessary for MRKP persistence, which was ruled out in our study. Subsequent analysis of the microbiota of treated mice and infected with MRKP revealed that mice did not recover their baseline microbiota after 1 month since the cessation of the antibiotic treatment. This result suggested, as previously was demonstrated for VRE (65) or C. difficile (24), that permanent changes in the microbiota could allow persistence of MRE in the intestinal tract. Both for VRE and C. difficile it was demonstrated that ampicillin or clindamycin promote permanent changes in the murine microbiota that render mice susceptible to colonization by these pathogens even 4 weeks after antibiotic withdrawal.

In order to investigate if antibiotics promote persistent changes in the microbiota that could promote MRKP colonization and persistence, mice were treated with AVN and allowed to recover for 2 or 4 weeks before inoculation of MRKP. Our results demonstrated that AVN produced profound changes in the microbiota that persisted up to 1 month post-treatment, which rendered mice susceptible to MRKP colonization. These results are consistent with those of Lewis et al. (139), in which it was 
demonstrated that mice treated with vancomycin or metronidazole remained susceptible to colonization with carbapenem-resistant $K$. pneumoniae during 2 weeks after the end of the treatment.

Although our results and those obtained by Lewis et al. (139) indicate that antibiotics per se can promote permanent changes in the microbiota, which permit gut colonization by MRKP and therefore could contribute to the ability of MRKP to persist for long time in the intestinal tract after antibiotic withdrawal, we cannot rule out the possibility that MRKP colonization could prevent the recovery of the microbiota, which would enhance persistence of the pathogen in the intestinal tract. Indeed, it has been shown in mice that infections with another member of the family Enterobacteriaceae (Salmonella) induced intestinal inflammation, which promotes microbiota dysbiosis, and enhances intestinal colonization by this pathogen (63). Thus, it would be important to investigate if MRKP, once colonizes the intestine, prevents the microbiota recovery and also enhances its own persistence in the murine gut.

Several studies have shown that the fecal transplant restores the microbiota and CR against intestinal pathogens. In a study published by Ubeda et al. (23) authors demonstrated that administration of fecal suspension from untreated mice to those that were previously treated with vancomycin and infected with VRE resulted in the recovery of the microbiota and clearance of VRE. We obtained similar results with MRKP. Indeed, mice treated with antibiotics that received a fecal transplant recovered a microbiota similar to that of untreated mice and were no longer susceptible to MRKP colonization. These results demonstrate that the incomplete microbiota recovery allowed MRKP colonization and that restoration of the microbiota with a fecal transplant allows reestablishment of CR against this pathogen. This last result shows the efficacy of the fecal transplant as a prophylactic therapy against MRKP colonization. Although, we did not investigate the therapeutic effect of fecal transplant on MRKP colonization by giving a fecal transplant to mice that were already colonized with MRKP, the therapeutic effect of fecal transplants against MRKP was already demonstrated by Caballero et al. (85). The authors demonstrated that fecal transplant could completely eliminate MRKP and VRE in mice that were already concurrently colonized with these 2 pathogens. Although our experimental set up was slightly different (treatment with a cocktail of antibiotics instead 
of treating with ampicillin; and mono-colonization with MRKP instead of MRKP+VRE), it is likely that similar results would have been obtained. However, this remains to be demonstrated.

In a study published by Ubeda et al. (23) the authors demonstrated that administration of an anaerobic fecal culture, besides fecal transplant, could clear VRE from the murine intestine. This experiment, in addition to next-generation sequencing analysis, allowed the identification of key commensal bacteria that provide CR against VRE. We decided to apply the same approach in order to determine those bacterial strains that could protect against MRKP colonization in mice. For this purpose, 4 different anaerobic fecal cultures (grown in Gut, Schaedler, Wilkinson and Sellers cultivation media) were administered to mice after the cessation of the AVN treatment. Notably, none of the tested anaerobic fecal cultures was able to prevent colonization with MRKP. This result was somewhat unexpected, since at least one of these media (Gut media), supposedly allows the growth of a remarkable proportion of the fecal microbiota, as described in a study published by Goodman et al. (141). However, it was possible that the Gut media, although could support the growth of a large group of intestinal bacteria, does not support the growth of key members of the microbiota necessary for the maintenance of the CR against MRKP. Indeed, subsequent sequencing analysis demonstrated that the levels of Barnesiella, one of the most abundant genera found in untreated mice, could not be restored in treated mice after administration of Gut media.

Since none of the anaerobic fecal cultures had a protective effect against MRKP, we decided to change the strategy in order to identify commensal bacteria that could confer protection against MRKP. We compared the microbiota compositions of mice treated with AVN after 1 month of recovery and untreated mice. This comparison lead to the identification of 5 genera that were the most affected by AVN treatment (Barnesiella, Alistipes, Bacteroides, Lactobacillus and Mucispirillum). Notably, administration of bacterial isolates from these 5 genera to mice treated with AVN partially restored CR against MRKP. Importantly, we also demonstrated that the total bacterial load in fecal samples from mice receiving the mix containing the 5 isolates did not differ from mice that received the fecal anaerobic cultures, which did not prevent MRKP colonization. These results demonstrated that the reduction in MRKP colonization levels in mice to 
which the mix containing the 5 isolates was given is not simply a result of restoration of the bacterial density. On the other hand, the effect of the protective bacterial mix was not the result of enhancing a recovery of the overall bacterial diversity, since the Shannon index in samples collected from mice to which the protective bacterial mix was given was the same as in those animals that did not receive this bacterial mix. Thus in this case, the mechanism by which the protective mix confer CR may differ from that one shown by Lawley et al. (100) for $C$. difficile. In their study, the authors were able to confer protection in mice against $C$. difficile by administering a mix containing 6 different commensal bacterial species. Notably, this bacterial mix was able to restore the microbial diversity observed in untreated/uninfected mice, which may inhibit pathogen colonization. Thus for that particular mix, restoration of the overall microbiota diversity could be crucial for conferring protection against the pathogen, which differs from the results we have obtained in our study.

In order to elucidate which of the 5 bacteria that were included in the mix could be responsible for the observed reduction in MRKP colonization levels, we analyzed the microbiota composition of mice treated with AVN and to which MIX5 was administered. Importantly, only the genera Barnesiella and Lactobacillus were significantly higher in the group of mice that received the protective bacterial mix, as compared to mice that did not receive the bacterial mix, suggesting that only these 2 genera were able to re-colonize the murine gut after the antibiotic treatment. This is surprising, since all of the administered bacteria were isolated from the mice feces, and thus are adapted to grow in the murine intestine. One possibility is that these bacterial strains need other commensal bacteria, absent from the intestine of the AVN treated mice, for colonizing the gut. Nevertheless, our results suggest that restoration of the levels of the genera Barnesiella and Lactobacillus is enough for conferring CR against MRKP intestinal colonization in mice. It is important to say that the genus Lactobacillus was also re-established in mice to which the Gut anaerobic fecal culture was given and these mice were not resistant to MRKP colonization. These results suggest that recovery of the genus Lactobacillus alone was not enough for suppression of the MRKP colonization. On the other hand, we cannot discard the possibility that the Lactobacillus strain supplied by the Gut mix was different from that one supplied by the MIX5. In addition, the Gut mix may contain other bacterial 
species that may enhance MRKP colonization. In any case, further research is needed to elucidate if the Barnesiella and Lactobacillus strains provided by the MIX5 are sufficient to provide protection against MRKP. In fact, we were planning to perform experiments in which mice will be treated with AVN and will receive Barnesiella and Lactobacillus strains in combination, as well as individually, but due to a deadline for thesis deposit at the University, we have not been able to include the results from these experiments in the thesis.

Interestingly, in our previous study we have shown that the presence of the genus Barnesiella in the murine intestine is associated with clearance of VRE from the murine gut, although the mechanisms of protection is unknown (23). In another study, $B$. intestihominis, together with 3 other bacterial strains, was shown to reduce the levels of the $C$. difficile colonization in mice (27). Importantly, members of the genus Barnesiella are well known as producers of the acetic acid (142), one of the most abundant SCFA in the intestine. Acetate has been shown to inhibit the growth of species from the Enterobacteriaceae family such as E. coli (143), suggesting a possible mechanism by which acetate produced by Barnesiella species could be providing protection against MRKP colonization in mice. On the other hand, many members of the genus Lactobacillus are known as probiotic strains. In a study published by Karska-Wysocki et al. (144) authors demonstrated that L. acidophilus and L. casei have inhibitory effects on the growth of methicillin-resistant Staphylococcus aureus (MRSA) in vitro. In another study the combination of the L. acidophilus and L. plantarum was shown to effectively reduce the multidrug resistant E. coli levels of colonization in mice intestine (145) However, the mechanism by which these probiotics confer protection in those particular studies remains unknown. On the other hand, previous studies suggested that some of the Lactobacillus strains could act against pathogens indirectly, through the modulation of the immune system, as seen in case of L.casei, whose administration to mice infected with S. typhimurium resulted in modulation of the inflammatory response and decreased the severity of Salmonella infection (146). On the other hand, many Lactobacillus strains encode for the bile salt hydrolase (BSH), enzyme that cleaves the amino acid side chain of glyco- or tauro- conjugated bile acids to generate unconjugated bile acids (cholic and chenodeoxycholic acids), which then are amenable to further bacterial modification to 
yield secondary bile acids (deoxycholic and lithocholic acid) (147). The secondary bile acids have been associated with the resistance to the $C$. difficile infection in mice (27), suggesting that, beside modulation of the immune response, some of the Lactobacillus strains could also act directly against pathogens, through production of the secondary bile acids. Moreover, some Lactobacillus strains are well known producers of bacteriocins. In a study published by Song et al. (148) authors described a novel bacteriocin produced by L. plantarum that exerted an in vitro inhibitory effect against $E$. coli. Although there are several possible mechanisms by which Barnesiella and Lactobacillus could exert their effect on MRKP colonization levels, further studies are needed to elucidate which mechanism is responsible for the protection against MRKP intestinal colonization. In fact, we performed in vitro experiments in which we tested if Barnesiella or Lactobacillus strains used in the MIX5 could inhibit directly MRKP. For now, we were not able to detect direct inhibition of MRKP by these protective bacterial strains in vitro. However, this does not necessary confirm that direct inhibition is not the mechanisms by which Barnesiella and/or Lactobacillus could perform their protective effect. Indeed, the metabolism of both strains could differ in vitro and in vivo. For this reason we are also planning to test the inhibitory effect of cecal contents from mice that received these two strains. In addition, metabolomic and transcriptomic studies from mice colonized with these two strains will be performed to investigate proteins and metabolites derived from these two strains that could have an effect on MRKP growth (either by direct inhibition or by depletion of specific nutrients).

We recognize that our study in mice has several limitations. One of them is that we did not test the protective effect against MRKP of every single commensal bacterium that was not recovered after AVN treatment. In any case, we were able to find at least some important key players in CR against MRKP. On the other hand, we have focused our studies on the bacterial fraction of the microbiota. Other studies have shown that the growth of pathogenic Enterobacteriaceae (i.e. enterohemorrhagic E. coli) could be inhibited by the fungi Saccharomyces cerevisiae in a dynamic gastrointestinal model (149). Thus, it would be interesting to analyze in future studies how changes in the mycobiome could have an effect on MRKP colonization and if reconstitution of the mycobiome with specific fungi is sufficient to confer CR. 
Overall, our results indicate that persistent microbiota dysbiosis, as a result of antibiotic treatment, promotes MRKP intestinal colonization in mice. Moreover, we were able to define changes in the microbiota composition after antibiotic treatment associated with the loss of the CR against MRKP. Importantly, we were able to demonstrate that the restoration of 2 bacterial genera, depleted by antibiotic therapy, was sufficient to significantly diminish the ability of MRKP to colonize the intestinal tract. 


\subsection{Microbiota dysbiosis and MRE colonization in hospitalized patients}

Infections caused by MRE represent a great threat worldwide, especially in hospitalized patients (81). They usually begin by the MRE colonizing the intestinal tract. Moreover, once established, MRE can reach high numbers in the intestine, which promotes their dissemination into the bloodstream (83). Woerther et al. (84) showed that carriers with the highest levels of ESBL-producing Enterobacteriaceae were also those with the highest risk of bacteraemia produced by these pathogens. Besides, high levels of colonization promote transmission of the pathogen among patients. Despite the importance, to this date no study has investigated risk factors that could be associated with MRE levels of gut colonization. In addition, not much is known about the members of the microbiota that are needed for maintenance of CR against MRE. In this second part of the thesis, we have investigated risk factors associated with MRE levels of colonization, and we have attempted to identify commensal bacterial species associated with resistance against MRKP intestinal colonization. For this study we analyzed the MRE levels and the composition of the microbiota of 817 samples collected from 140 acute leukemia patients, a population that is frequently colonized with MRE. Interestingly, our results show that more than a half of the analyzed patients $(60 \%)$ got colonized with MRE during their stay at the hospital. A previous study in which the authors collected fecal samples from patients with haematological and oncological malignancies within $72 \mathrm{~h}$ of their admission to a hospital revealed that $17.5 \%$ of these patients was colonized with ESBL-producing Enterobacteriaceae (76). In another study, authors reported $35 \%$ of prevalence of CRE in fecal swabs collected on the day 4 since the admission in patients from the intensive care unit (78). Although our results show a higher percentage of patients colonized with MRE than the one reported in these 2 studies, it could be explained by the fact that we followed our patients through the whole admission period, which allowed us to detect MRE in cases when patients got colonized later during their stay in the hospital, while in previously mentioned studies screening was performed exclusively on third or fourth day after patients' admission.

The analysis of the susceptibility pattern of the isolated MRE strains has revealed some interesting and clinically useful data. First, most of the isolated MRE 
strains identified in acute leukemia patients are resistant to ciprofloxacin. Ciprofloxacin, a second generation fluoroquinolone, is given to these patients from the moment of admission to the hospital as prophylaxis, and thus, treatment with this antibiotic could favor the expansion of the ciprofloxacin-resistant MRE strains in the intestine of the studied acute leukemia patients. Indeed, Jorgensen et al. (150) demonstrated that exposure of Pseudomonas aeruginosa to ciprofloxacin rapidly selects for mutants with high-level ciprofloxacin resistance. Moreover, in a review published by Redgrave et al. (151), the authors point to the high correlation between consumption of the fluoroquinolones and percentage of E. coli strains resistant to these antibiotics. Thus, at least for the acute leukemia patients of the Hospital La Fe, treatment with ciprofloxacin will not be recommended as a therapy against infections produced by MRE. In contrast, most of the isolated MRE strains were sensitive to amikacin and tigecyclin, 2 antibiotics that have been successfully used for the treatment of MRE, including carbapenemresistant strains of K. pneumoniae (81). For this reason, the combination of amikacin and tigecycline could be a promising treatment for elimination of MRE in these particular patients.

As previously mentioned in the Introduction, all studies to date have analyzed the association of different clinical factors with the MRE colonization, by looking at prevalence of MRE positive samples (presence or absence). Here, for the first time, we attempted to identify clinical and other factors associated with the levels of the MRE intestinal colonization, since high MRE intestinal levels are crucial for subsequent dissemination to the bloodstream, which can indeed put in serious threat the life of the patient. Importantly, we found that gender, age, type of leukemia or parenteral nutrition do not affect MRE colonization levels. However, the MRE colonization levels were significantly higher in samples collected during the chemotherapy compared to those samples collected during the transplant period. The major difference between these 2 admission periods is that during the transplant period, patients receive, beside chemotherapy to eliminate the cancer cells, a bone marrow transplant containing immune cells precursors. Thus, the lower levels of MRE in samples obtained during the transplant period could be due to a faster re-establishment of a proper immune response, which could contribute to diminishing MRE levels in the gut. The only immune parameter 
available for these patients was the level of neutrophils in blood. Surprisingly, our results suggest that neutropenia could be associated with the decrease in MRE colonization levels. This could be due to the more intensive antimicrobial therapy that neutropenic patients received. Indeed, as discussed below, we found a clear association between administration of beta-lactams with activity against MRE and decrease in MRE levels. When we excluded samples in which the MRE was sensitive to the antibiotic that the patient was receiving, the observed neutropenia was still associated with decrease in MRE levels, although to a lower extent, suggesting that indeed treatment of antibiotics could explain in part the decrease in MRE levels observed during neutropenia. Nevertheless, it should be further investigated why neutropenia is still associated with lower MRE levels after excluding samples in which the MRE is sensitive to the antibiotic that the patient was receiving. Besides neutrophils, other immune cells and effectors molecules, which are known to diminish pathogen load (e.g. IgA) could have a role in MRE intestinal colonization in leukemia patients. This information was not available for the patients under study, but it will be interesting to investigate in future studies how different immune effector cells and molecules affect MRE levels in the intestinal tract of patients. Another possible explanation for the difference in MRE intestinal levels between samples collected during chemotherapy and transplant period could be that certain patients receiving a specific type of transplant would be more resistant to MRE. Two main types of transplants are received by these patients, depending on the origin of the cells infused (autologus or allogeneic, depending if the donor and recipient are the same or are different subjects). Nevertheless, patients receiving both types of transplants have similar MRE intestinal levels, suggesting that the type of transplant does not seem to be a key factor involved in MRE intestinal colonization, neither the type of chemotherapy as we also demonstrated. Future studies, including the analysis of other immune parameters, besides neutrophils, will be required to clarify the differences observed during both admission periods.

Various studies have identified different antibiotic treatments, among which are aminoglycosides, carbapenems, quinolones and colistin, as risk factors for the presence of antibiotic-resistant Enterobacteriaceae in the gut $(76,78,86,152)$. These studies, as the ones obtained in our mouse experiments, suggest that disruption of the CR by antibiotic 
treatment opens the way to colonization with MRE. However, our analysis of MRE levels in samples collected from acute leukemia patients revealed that beta-lactam treatment produces a reduction in MRE colonization levels. Moreover, when we analyzed only those samples in which the identified MRE strains were sensitive to betalactams, the reduction in colonization levels was even more pronounced. More importantly, the administration of beta-lactams, when the identified MRE strains were resistant to these antibiotics did not produce an expansion of the pathogen. Thus from our analysis, we could conclude that treatment with beta-lactams does not promote intestinal colonization with MRE but rather diminish the sensitive strains inhabiting the gut. This result contradicts the result obtained in our mouse model in which mice that were receiving ampicillin (a beta-lactam antibiotic) were highly susceptible for MRE colonization. In order to understand this contradictory result, it is important to notice that all acute leukemia patients receive oral prophylactic treatment (ciprofloxacin) from the first day of admission to the hospital. This antibiotic is known to produce alterations in the microbiota composition, which could disrupt CR (106). Thus, it is possible that betalactam treatment, which is given after ciprofloxacin treatment, does not produce any further alteration in the microbiota that could increase the level of MRE colonization. On the other hand, it is possible that beta-lactams does produce some changes that promote MRE colonization in some but not in all patients and therefore we are not able to detect a significant effect. Interestingly, the beta-lactams that the patients received were administered through the intravenous route. Thus, our results demonstrate that, although administered systematically, beta-lactams could reach the intestine at enough concentration to reduce the MRE colonization levels, in case the strain is sensitive to beta-lactams. Overall, our results suggest that the use of beta-lactams in acute leukemia patients could be beneficial for combating MRE infections. Administration of these antibiotics, when the patient is colonized with an MRE susceptible to beta-lactams would result in MRE decrease, which would reduce the probability of MRE dissemination into the bloodstream, while the administration when patient is colonized with a beta-lactam resistant strain would not promote MRE expansion. Nevertheless, it is important to say that the administration of beta-lactams when patient is colonized with MRE strain that is sensitive to these antibiotics could lead to appearance of beta-lactam resistant strains. In 
our study we detected change in beta-lactam resistance pattern in 2 cases out of 39 to which beta-lactam was administered, while no change from sensitive to resistant MRE strain was detected in the absence of beta-lactam treatment. This result suggest that the administration of beta-lactams could select for the expansion of low abundant MRE strains resistant to beta-lactams, or could result in change of the resistance in existing MRE strains. More importantly, we detected an increase in MRE intestinal levels in samples after the switch in resistance to beta-lactams in MRE detected strains. These results suggest that there should be especial caution when prescribing the beta-lactam treatment to acute leukemia patients, since it could favor the expansion of MRE strains resistant to beta-lactams, although the change in beta-lactam resistance does not seem to occur frequently. On the other hand, we observed that aminoglycoside treatment (i.e. amikacin) in general does not seem to produce change in MRE colonization levels. However, our results shown that intravenous aminoglycosides have an inhibitory effect on MRE intestinal levels when given to patients colonized with strains sensitive to these antibiotics. Due to the low number of samples in which the identified MRE was resistant to aminoglycosides, we were not able to test the effect of administration of this antibiotic to patients colonized with aminoglycosides-resistant MRE strains, thus we cannot rule out the possibility that aminoglycosides may promote microbiota changes that may enhance colonization of MRE aminoglycosides-resistant strains. Importantly, our results shown that the glycopeptide treatment reduces MRE intestinal levels. Glycopeptides analyzed in this study were vancomycin and teicoplanin, antibiotics to which MRE strains are resistant. Thus, it was somewhat surprising that glycopeptides could affect MRE colonization levels. In order to discard that this effect was due to administration of other antibiotics at the same time as glycopeptides, to which the identified MRE strain was sensitive, analysis was repeated only with those samples in which the identified MRE strains were resistant to all antibiotics that were administered to the patient at the moment of sampling. Although the number of analyzed pairs of samples was small (3), the analysis did not reveal significant change in MRE levels as a result of glycopeptide treatment. In summary, the analysis performed indicate that systemic antibiotics, administered to a patient colonized with an MRE that is sensitive to that particular antibiotic, are able to reach sufficient levels in the intestinal tract to diminish intestinal 
MRE levels. On the other hand, our results show the importance of including data about susceptibility patterns of the analyzed pathogens into the analysis and design of similar studies.

On the other hand, we have shown that none of the antifungal treatments analyzed in our study seem to affect MRE intestinal levels. However, some authors already reported that Saccharomyces cerevisiae significantly reduced E. coli O157:H7 growth in a dynamic multi-compartmental digestion system, possibly through a potential inhibitory role of ethanol produced by the yeast (149). Thus, the fungal microbiota could have a protective role against MRE. Nevertheless, as in case of beta-lactam treatment, it is possible that the microbiota of acute leukemia patients is already perturbed as a result of prophylactic ciprofloxacin treatment, and therefore antifungals do not promote more expansion of MRE. Nevertheless, it would be interesting to investigate the composition of the fungi in the intestinal microbiota and determine if some of them could have protective role against MRE intestinal colonization.

Finally, the multivariate analysis revealed 2 factors independently associated with the change in MRE colonization levels: administration of beta-lactams when the identified MRE strains are beta-lactam-sensitive was independently associated with decrease in MRE levels; and the taxonomy of the pathogen (the genus Klebisella) was associated with higher MRE levels. Since we have demonstrated that administration of beta-lactams and aminoglycosides (piperacillin-tazobactam, carbapenems, amikacin) can have an inhibitory effect against MRE, it was possible that the MRE strains within the genus Klebsiella have a higher prevalence of resistance to those beta-lactams or aminoglycosides, which will promote their colonization of the intestinal tract of acute leukemia patients. For this reason, we analyzed the percentage of Klebisella MRE strains resistant to beta-lactams and aminoglyosides and compared it to the percentage of other MRE resistant strains (e.g. Escherichia). There was no difference in resistance prevalence among the different MRE analyzed, suggesting that the higher colonization levels of Klebsiella MRE are not due to a higher resistance to the beta-lactams or aminoglycosides administered to patients.

Besides the inhibitory effect of antibiotics on MRE intestinal colonization, once the MRE has colonized the gut, our results also demonstrate that mucositis in the first 2 
weeks previous MRE colonization was associated with increased MRE intestinal levels. Mucositis is a negative consequence of the chemotherapy, characterized by inflammation, tissue damage and subsequent ulceration of the oral and gastrointestinal mucosa due to the cellular damage of epithelial cells. Importantly, it has been demonstrated in mice that intestinal inflammation can promote a dysbiotic state of the intestinal microbiota, which would favor colonization with intestinal pathogens (153). Due to the low number of patients with mucositis and the variability of the microbiota among patients, we were not able to elucidate if mucositis was associated with specific changes in the microbiota that could promote MRE colonization. Taking into account a recent study reporting differences in the composition of the oral microbiota depending on the mucositis severity (154), it is likely that mucositis could also produce changes in the gut microbiota, which may impact MRE colonization levels. Further studies including more samples from patients developing mucositis should be necessary in order to elucidate a possible link between mucositis, microbiota and MRE colonization.

Overall, we were able to identify factors associated with the MRE colonization levels in the intestinal tract of acute leukemia patients. As previously mentioned, high levels of colonization with MRE is associated with the increased risk of bacteremia produced by these pathogens. Thus, our results could be useful for the prevention of dissemination of MRE into the bloodstream. According to this, we are currently collecting data about the bacteremias that occurred in the acute leukemia patients included in this study in order to confirm, in our patient's cohort, that the high MRE colonization levels increase the risk of subsequent bloodstream infection.

Besides identifying clinical factors associated with the MRE colonization, we wanted to investigate how changes in the microbiota composition may impact the ability of MRE to colonize the intestine, which would finally allow us to identify commensal bacterial strains that could provide protection against MRE colonization. Due to the complexity of data we decided to apply a recently published LV mathematical model (127). This model was successfully applied on microbiome data obtained from patients colonized with $C$. difficile, identifying $C$. scindens as a protector commensal bacterium against infections produced by $C$. difficile (27). This model takes into account both clinical factors and microbiota data in order to identify which commensal bacteria and 
clinical factors impact pathogen levels. Interestingly, when applied on the microbiota dynamics at the genera level, LV confirmed administration of beta-lactams, when the detected MRE strains are sensitive to the beta-lactams that are received by the patient, as a factor that is negatively correlated to MRE levels. Interestingly, LV selected chemotherapy (positively correlated) and transplant (negatively correlated) as clinical factors associated with MRE intestinal levels. Although these two factors did not pass the previous multivariate analysis, it is possible that chemotherapy and transplant have a strong effect over the intestinal microbiota, which affects MRE colonization levels, and thus are being selected by LV. Among positively correlated factors, LV selected 2 bacterial genera: Paraprevotella and Klebsiella. The positive correlation of the genus Klebsiella is in agreement with the results obtained in the previous multivariate analysis, where colonization with MRE strain classified as Klebsiella was also positively correlated with MRE levels. Further studies will be required to elucidate if this positive association could be due to the better adaptation of this genus to the colonization of the intestinal tract. On the other hand, we found a negative correlation of the genus Coprobacillus with MRE levels, which suggests that commensal bacterial species within this genus could have a protective role against MRE colonization in acute leukemia patients. Importantly, the genus Coprobacillus was found to be negatively associated with $C$. difficile when LV model was applied on data obtained in a study using a mouse model (127). Moreover, in agreement with our results, in this study, Coprobacillus was negatively correlated with bacteria from the family Enterobacteriaceae. Coprobacillus is a relatively novel genus, isolated and characterized for the first time in 2000 (155). Coprobacillus are Gram-positive non-spore forming bacteria that reside in the intestinal tract. Their role in colonization resistance have not been studied yet, but interestingly, a very recent paper that analyzed metagenomic sequences searching for lantibiotics (small peptides with bactericidal activity) reported novel lantibiotic genes which were encoded by 2 members of the Coprobacillus genus (156). Although lantibiotics are mainly effective against Gram-positive bacteria, recently, a lantibiotic with activity against Enterobacteriaceae pathogens, including Escherichia and Salmonella, has been identified and patented (US Patent Application 20110305763). This lantibiotic is produced by Bifidobacterium longum, a Gram-positive natural inhabitant of the gastrointestinal tract. 
Right now, in collaboration with the group of Dr. Jean Marc Rolain, experts in culturomics (massive isolation of commensal bacteria), we are trying to isolate bacterial strains belonging to the Coprobacillus genera in order to test their protective effect against MRE.

In an attempt to identify with more precision those commensal bacteria that could protect against MRE colonization, we decided to apply LV at the level of OTUs (sequencing approach to identify bacterial species). As expected, all clinical variables that were previously selected through LV as correlated with MRE levels were still significant when introducing in the model the microbiota information at the OTU level. In addition, 1 OTU within the genus Klebsiella was positively associated with MRE levels, confirming the results obtained when LV was applied at the genus level. This positive association can be explained in several ways: (i) an expansion of certain endogenous species of the genus Klebsiella promoted the colonization of MRE strains, or more likely (ii) a particular MRE species from the genus Klebsiella is highly capable of colonizing the intestinal tract and its levels can be identified both by $16 \mathrm{~S}$ rRNA sequencing and CFU counts, which actually positively correlate because they are indeed the same bacterial species. Future genome sequencing analysis of the Klebsiella MRE isolated strains in comparison with metagenomic analysis of the fecal samples could reveal if the Klebsiella observed through culture and 16S rRNA sequencing is the same or a different strain. If it was found that a particular Klebsiella species or strain is more capable of colonizing the intestinal tract, subsequent studies should define the specific colonization factors that allow this pathogen to better colonize the intestine. In addition to the Klebsiella OTU, a higher abundance of an OTU belonging to the genera Alistipes was associated with an increase of MRE levels. Interestingly, Alistipes have been associated with the appearance of inflammation in pediatric patients with Irritable Bowel Syndrome (157), which, as indicated above, could promote the expansion of certain pathogens included in the family Enterobacteriaceae. Moreover, higher levels of Alistipes, associated with increased levels of the family Enterobacteriaceae, have been also observed in other dysbiotic conditions, such as major depressive disorder (158). Obviously, additional experiments must be performed, including the isolation of Alistipes strains in combination with mouse models of infection in order to elucidate if 
Alistipes has any role in the expansion of MRE strains. Several bacterial OTUs were identified by LV model to be negatively associated with MRE. These include: 1 OTU within the genus Lactobacillus and 2 OTUs within the genus Bacteroides. Notably, none of these OTUs belong to the genus Coprobacillus previously selected by the LV model. However, it is possible that the genus selected as significant combines different Coprobacillus OTUs, not very prevalent among the different patients included in the study, that share similar mechanisms by which they exert their effect on MRE. Thus, none of these OTUs could be identified by LV to be associated with a decrease in the MRE levels, but combined in the same genus, they could indeed be identified by LV as bacteria that are negatively associated with MRE levels. In contrast, we did not identify a negative association between some genera (e.g. Lactobacillus) and levels of MRE, but we did identify a negative association between specific Lactobacillus OTU and MRE levels. In this case, some OTUs selected by LV could affect MRE colonization levels (i.e. Lactobacillus OTU20), however, when analyzed together with other OTUs from the same genus, this association would be weaker (not significant) if the other OTUs within the same genus do not have a negative impact on MRE. As mentioned in the previous chapter, bacteria belonging to the genus Lactobacillus, in combination with Barnesiella, could provide $\mathrm{CR}$ against $\mathrm{MRE}$ in our mouse model of infection. Nevertheless, as mentioned above, we still need to verify the contribution of the isolated murine Lactobacillus strain on protection against MRE colonization. In the case of the identified human Lactobacillus OTU, we are right now trying to isolate strains from this OTU in order to test their protective effect against MRE using in vitro and mouse models. As previously mentioned, strains within the genus Lactobacillus (e.g. L. plantarum) have been identified as probiotics. Although we were able to determine more precisely, using the 16S rRNA information, which Lactobacillus species were associated with the decrease in MRE levels in the LV model, due to the legal questions concerning patents, we cannot indicate to which species the Lactobacillus OTU20 belongs to. On the other hand, LV also selected 3 OTUs within the genus Bacteroides, 2 of them being negatively associated with MRE intestinal levels, and 1 positively. These results are in agreement with the idea that we explained above that not all OTUs from the same genus have to be associated in the same way with MRE colonization. Interestingly, species from the genus 
Bacteroides have been shown to have both a positive and negative effect on intestinal colonization by Enterobacteriaceae pathogens. Huang et al. (159) demonstrated that commensal members of the genus Bacteroides have the capacity of liberating sialic acid from the intestinal tissue, which promotes the outgrowth of $E$. coli during inflammation. On the other hand, Kamada et al. (160) demonstrated that when mice were fed with a simple sugar diet, Bacteroides competes for nutrients with $C$. rodentium, which results in elimination of this pathogenic bacteria from the murine intestine.

Overall, we were able to identify clinical factors associated with changes in MRE colonization levels. More importantly, we identified several commensal bacteria that were negatively associated with MRE colonization levels in acute leukemia patients. However, as mentioned above, further studies are needed in order to fully elucidate the role of these bacteria in protection against MRE colonization. Right now, thanks to a collaboration with Dr. Jean Marc Rolain, expert in culturomics, we are trying to isolate the commensal bacteria identified to be associated with decrease in MRE rates in order to validate their protective effect against MRE. The capacity of these human commensal isolates to inhibit MRE will be tested using GF mouse models in collaboration with Dr. Karina Xavier, principal investigator of the Gulbenkian Insitute, which harbors one of the biggest GF mice facilities in Europe. Results from these future experiments, may identify novel commensal bacterial strains in that could be used as probiotics to confer protection against MRE. Finally, we would like to mention that taking into account all the microbiota and clinical data that we have obtained, we can indeed perform additional studies, which due to the limitation in time to complete this thesis, could not be included here. Among these studies, we plan to analyze how different clinical variables (i.e. antibiotics, mucositis) impact the microbiota composition. In addition, we are performing analysis in order to evaluate how changes in the microbiota impact another disease that frequently occur in acute leukemia patients (i.e. Graft versus Host Disease). 


\subsection{The role of the microbiota dysbiosis in ERE}

Epizootic rabbit enteropathy is a devastating disease that occurs in young rabbits after the weaning, and that has been associated with microbiota dysbiosis (89). In order to investigate the role of the microbiota dysbiosis on ERE development, caecotroph samples were collected. Caecotroph samples, type of feces made up of cecal contents and secreted by rabbits at dawn, are believed to be microbiologicaly more similar to the cecal content than normal type of feces (161), although no study to date confirmed this. Importantly, since the inoculation of the cecal contents has been shown to reproduce the ERE in specific pathogen free healthy rabbits, it is of interest to investigate if analysis of the microbiota present in caecotrophs could be extrapolated to the cecum. Here, for the first time, we used high throughput sequencing to investigate the composition of the microbiota in caecotrophs, and to compare it to the microbiota from the cecal content of the rabbit. Importantly, we could confirm that the microbiota composition of caecotroph samples is representative of the microbiota of cecal contents, indicating that the sampling of caecotrophs could be useful for determination of the microbiota composition in cecum, avoiding the sacrifice of the animals.

Once we confirmed that caecotrophs could be used for identification of changes in the microbiota composition in cecum, we proceeded with characterization of the microbiota dysbiosis observed after the ERE onset. A study published by Bauerl et al. (89) described reduced diversity and alteration of the microbiota composition in rabbits with ERE. The results from this study show that rabbits with ERE develop a pronounced intestinal dysbiosis, characterized mainly by the expansion of several bacterial genera. Consistent with the results from the study published by Bauerl et al., ERE rabbits from our study harbored a microbiota with lower diversity and altered abundances of several bacterial taxa, including increased levels of Akkermansia, Bacteroides and Clostridium. The higher abundance of the genus Akkermansia, a mucin-degrading bacterium, could be due to the presence of the large amounts of mucus in the intestine of ERE rabbits. Interestingly, the genus Alistipes was found decrease in ERE rabbits in the study published by Bauerl et al., while in our study this genus had increased abundances in rabbits developing the disease. Although the reasons for this discrepancy are unknown, it 
is possible that different species from the genera Alistipes, present in the rabbits from these 2 studies, may behave differently after ERE initiation. Alternatively, different bacterial taxa present in the different rabbits may influence Alistipes levels after ERE initiation.

Bauerl et al. defined 2 types of ERE, inflammatory and secretory, based on the type of genes upregulated in each of the groups (cytokines or mucins, respectively) and the microbiota changes that occur after ERE initiation. Interestingly, samples from ERE rabbits obtained in our study could be separated by their different microbiota in 2 groups along the first PCoA axis. However, since we did not analyze the genes expressed by the host, we were not able to confirm if this separation was associated with different host gene expression profiles.

We and others have shown that the microbiota of ERE rabbits is characterized by dysbiosis (89). However, it remains unclear whether the microbiota changes observed in ERE rabbits were the cause or the consequence of the disease. As mentioned in the Introduction section, ERE occurs shortly after weaning, and the weaning produces drastic changes in the microbiota composition (18). Thus it was possible that rabbits that develop ERE could have different microbiota changes after weaning that could promote ERE. In order to investigate how microbiota of rabbits evolves after the weaning, and if some of the changes in the microbiota that occur during this period could result in the development of ERE, samples were collected from littermate rabbits the day of weaning, the day after weaning and then every other day during the next 20 days. Interestingly, our results show that the microbiota of rabbits the first day of weaning is very variable among animals, and changes over time towards a microbiota that is more similar among different rabbits. Moreover, the analysis of the overall microbiota structure revealed that the microbiota of young rabbits reaches a composition more similar to the one of adult rabbits (mothers) on the $11^{\text {th }}$ day after weaning, suggesting that the transition to the solid food in rabbits results in rapid adaptation of the intestinal microbiota. Importantly, the evolution of the overall microbiota composition between healthy rabbits and those that will develop ERE did not differ, indicating that gross changes in the microbiota composition as a result of the weaning do not promote ERE development. Moreover, when samples were analyzed using the 454 platform, no specific changes in the 
microbiota composition, observed in ERE rabbits, could be detected before the disease onset, suggesting that the dysbiosis is rather the consequence of the disease and not the cause.

Importantly, Licois et al. (92) reproduced the disease in healthy animals by administrating intestinal contents obtained from rabbits affected by ERE, suggesting that a pathogenic agent could be involved in the development of this pathology. An infectious process is consistent with the results that we obtained. In the first stages of the infectious process, the pathogen could represent a very small fraction of the whole bacterial populations residing in the intestinal tract, thus the microbiota structure of healthy and sick rabbits could be equivalent before ERE onset. Moreover, the previous analysis was performed on the data obtained by 454 sequencing, and the changes in low abundant bacteria could have been undetected due to the relatively low number of sequences obtained by this technology ( $2849 \pm 779$ sequences per sample). For this reason, and in order to investigate if there was any alteration in the abundances of low abundant bacteria between ERE and healthy rabbits, all samples were re-sequenced using the newer Illumina platform that allows obtaining a higher sequencing coverage. Analysis performed on sequences obtained by Illumina revealed results similar to those obtained by 454 platform. The dysbiosis of the microbiota identified in ERE rabbits was not present in these rabbits before the disease onset. However, the day of disease onset ERE rabbits had significantly higher abundance of the genus Clostridium. This genus could not be detected the day of onset in ERE rabbits using the 454 platform because of its low abundance at this particular day $(0.022 \%)$. Importantly, the increase in the genus Clostridium was due to the higher presence of a unique OTU (OTU31), defined as a novel species of Clostridium-Clostridium cuniculi, phylogenetically related to $C$. botulinum and $C$. perfringens, 2 well known pathogens. Importantly, $C$. cuniculi could not be detected in any of the healthy animals. On the other hand, this OTU expanded in all ERE rabbits after the ERE onset. These results suggest that $C$. cuniculi could be the trigger of the ERE, rather than the overall microbiota dysbiosis observed in ERE rabbits.

As explained previously, in some cases, microbiota dysbiosis is needed for the colonization of the intestinal tract with pathogens. In a study published by Buffie et al. (24) authors demonstrated that the profound microbiota dysbiosis as a result of 
clindamycin treatment promotes the colonization of the mice intestine with $C$. difficile. However, results from our study indicate that no previous dysbiosis seems to be required for the colonization and expansion of $C$. cuniculi in the intestinal tract of rabbits. Similar result were obtained in a study published by Barman et al. (88), in which the authors demonstrated that a Salmonella strain can colonize the intestine of mice presenting an intact microbiota. In this case, intestinal colonization with Salmonella produced inflammation and profound changes in the microbiota of mice, which was exploited by Salmonella for its own expansion. In the same way, the dysbiosis that occurs upon ERE initiation may promote the expansion of $C$. cuniculi. Future studies are required in order to validate this hypothesis and understand the molecular mechanisms of it. Our results not only revealed an etiological candidate for ERE development, but also point to the importance of the analysis of low abundant bacteria, that was provided by the higher coverage obtained by Illumina sequencing technology. This last point should be taken into account in the methodological design of other novel studies to investigate the role of microbiota changes in disease development.

A recent study has shown that restriction in food consumption results in marked microbiota dysbiosis in rats (162). Since a decrease in food consumption was the first sign of ERE, an experiment was performed to investigate if the observed microbiota dysbiosis in ERE rabbits could be in part due to the decrease in the food consumption. The analysis revealed higher presence of the genera Anaerovorax and Olsenella in rabbits after 2 days of food restriction, while levels of the genus Anaeroplasma were lower in these animals compared to those that had ad libitum access to food. None of these changes were observed in a study performed on rats. Nevertheless, the difference in the results could be due to a different baseline microbiota in rabbits and rats. Importantly, the microbiota dysbiosis observed in ERE rabbits was not detected in the group of rabbits subjected to the food deprivation. Specifically, only the higher abundance of the genus Anaerovorax was observed in both samples collected from ERE rabbits after the disease onset and those samples collected after the food deprivation, indicating that only alteration in abundance of this particular genus in ERE rabbits could be explained by the decrease in the food consumption. On the other hand, our results shown that the decrease 
in food consumption was not the trigger of ERE development, since food restriction did not promote to a higher extent the development of ERE signs.

Since the role of the food deprivation as a promoter of the microbiota dysbiosis and ERE was discarded, investigation was centered on the novel Clostridium strain. Importantly, various authors have associated pathogens within the genus Clostridium with the ERE. As previously mentioned, Marlier and coworkers (94) detected high fecal counts of $C$. perfringens in the digestive contents of $80 \%$ of the ERE field cases. On the other hand, Romero et al. (95) showed that high counts of $C$. perfringens in the hindgut are associated with clinical symptoms of ERE and mortality. Interestingly, Equine Grass Sickness (EGS), another gastrointestinal disease which occurs in horses and results in similar lesions as those observed in ERE rabbits, has been associated with the presence of $C$. botulinum and C. perfringens (163). Although the etiological agent of EGS remains unknown, it was proposed that a putative toxin, ingested by the horses, produces neuronal damage leading to the stasis of the intestinal transport in EGS. Importantly, some authors have suggested that the paresis in the intestinal transport could be the cause of the pathological changes observed in ERE rabbits (92). For this reason, analysis of the genome of $C$. cuniculi was performed in order to identify potential virulence factors, including toxins that could promote ERE. Interestingly, we detected the presence of an operon strikingly similar to the operon encoding the type A neurotoxin complex in $C$. botulinum. The botulinum toxin inhibits the release of neurotransmitter acetylcholine from peripheral cholinergic synapses, resulting in the muscle relaxation and paralysis. However, and despite the presence of most of the other genes characteristic for this operon (e.g. hemagglutinins), the gene encoding the botulinum toxin was absent from the operon detected in C. cuniculi. Nevertheless, in place of the botulinum toxin, the operon encodes for a protein with a domain similar to the one detected in serine protease inhibitors. Acetylcolinesterase is a serine protease that catalyzes the breakdown of acetylcholine, and its inhibition could lead to paralysis, which could be compatible with the cause of some of the signs observed during ERE. On the other hand, a recent study has demonstrated that the non-toxic components of the botulinum operon (hemagglutinins) could form a complex that has in vivo toxicity on the small intestine of the rats (164). The authors have shown that non-toxic botulinum complex induces the 
formation of the vacuoles. Interestingly, vacuole formation was also observed in the small intestine of the ERE rabbits (165). These results suggest possible mechanisms by which C. cuniculi could be involved in ERE development.

In order to test the role of $C$. cuniculi in ERE development, an experiment was performed in which healthy SPF rabbits were infected with the putative etiological agent of ERE. Since it has been demonstrated that ERE can be reproduced by the administration of the intestinal content of sick animals, cecal contents of ERE rabbits were administered to a second group of rabbits as control. None of these 2 groups developed the ERE signs, although a transitory reduction in food consumption was observed in rabbits that received the cecal contents from sick rabbits. Our failure in the reproduction of ERE using cecal contents from sick animals could be due to a higher resistance to the disease of the rabbits used in this experiment as compared to those used by Licois and colleagues (92). In addition, our inoculum may contain lower amounts of the ethological agent required for the development of ERE as compared to the well characterized TEC inoculum used for the reproduction of ERE in previous studies $(92,93)$. Similar reasons could explain the failure of C. cuniculi to promote ERE. Another possible explanation is that species $C$. cuniculi includes various strains with different levels of virulence, and that the isolated representative, used in experiment in which we attempted to reproduce ERE, belongs to one of the low-virulent strains. However, we cannot rule out the possibility that other bacteria, besides $C$. cuniculi, are required for the development of ERE, or that C. cuniculi is just one of the first commensal bacteria to expand after the initiation of the disease, but not its cause. Further experiments should be performed in order to fully elucidate the role of $C$. cuniculi in the ERE development.

As mentioned in the Introduction, microbiota dysbiosis has been associated with various diseases, including IBD (69), diabetes (70), asthma (71), etc. However, for some of them, it remains unclear whether the observed dysbiosis is the cause or the consequence of the disease. Our results demonstrate the importance of prospective longitudinal studies for investigating the role of the microbiota dysbiosis in disease, since the overall microbiota dysbiosis observed in ERE rabbits was not the trigger of the ERE, but rather its consequence. Nevertheless, we cannot discard that the dysbiosis observed after ERE onset could indeed play a role in the progression of the disease, although not 
in its initiation. In fact, a previous study showed that treatment of rabbits with the antibiotic bacitracin does not prevent disease initiation but diminishes the mortality rate associated with ERE (166), suggesting that certain intestinal bacteria could play a role in disease exacerbation. Regarding this last point, an expansion of the genus Campylobacter was observed in most of the rabbits that develop ERE. Species belonging to this bacterial genus can produce diarrhea, a sign that is frequently observed in rabbits that develop ERE. Thus further experiments should also elucidate if this particular bacterium could be playing a role in the progression of the disease.

In summary, we have identified a single microbiota change preceding the development of ERE signs (presence of $C$. cuniculi), although further research is required to fully elucidate its role on ERE development. 



\section{Conclusions}

1. Oral administration of ampicillin, vancomycin and neomycin affects differently the intestinal microbiota of mice, which is then reflected on the capacity of multidrug resistant Klebsiella pneumoniae (MRKP) to colonize the gut of mice treated with these antibiotics. Ampicillin and vancomycin induce drastic and persistent changes in the microbiota composition, which allowed gut colonization and persistence by MRKP. However, neomycin induces minor microbiota changes, which did not allow intestinal colonization by MRKP. The combination of the 3 antibiotics (AVN) promotes intestinal MRKP colonization and persistence to a higher extent that any of 3 antibiotics alone.

2. The murine intestinal microbiota does not recover even after 1 month of AVN withdrawal. These persistent changes render mice permanently susceptible to MRKP colonization after AVN treatment and facilitate MRKP persistence in the intestinal tract after antibiotic withdrawal.

3. Fecal transplant can restore the baseline microbiota of mice after antibiotic withdrawal, which prevents MRKP intestinal colonization. In addition, the administration of 5 commensal bacterial strains, belonging to genera that did not recover after antibiotic withdrawal (Barnesiella, Alistipes, Bacteroides, Lactobacillus and Mucispirillum), partially restored colonization resistance against MRKP in antibiotic-treated mice. The protective effect of the commensal mixture is not due to restoration of total bacterial density or diversity.

4. Univariate analysis of clinical factors in relation with multidrug resistant Enterobacteriaceae (MRE) intestinal levels in hospitalized patients revealed that: (i) acute leukemia patients admitted in the hospital for receiving chemotherapy are colonized with higher MRE levels than those patients admitted for receiving a transplant; (ii) MRE intestinal levels decrease after systemic administration of beta-lactams and aminoglycosides when the identified MRE strain is sensitive to these antibiotics; (iii) however, administration of beta-lactams does not promote the expansion of MRE strains resistant to the beta-lactams received.

5. Multivariate analysis applied on all factors tested in univariate analysis identified beta-lactam treatment, when the identified MRE strain is susceptible to the 
administered beta-lactams, as an independent factor associated with decrease in MRE intestinal levels. In addition, the phylogeny of the MRE (i.e. being classified within the genus Klebsiella) was identified as an independent factor associated with higher MRE intestinal levels.

6. Linear regression analysis identified the development of mucositis prior to MRE detection as a clinical factor associated with higher MRE intestinal levels.

7. Lotka-Volterra (LV) model applied to clinical and fecal microbiota sequencing data obtained from acute leukemia patients identified higher abundances of the genus Coprobacillus to be associated with a decrease in MRE fecal levels, while higher abundances of the genera Paraprevotella and Klebsiella were associated with an increase in MRE fecal levels. In addition, higher abundances of an operational taxonomical unit (OTU) within the genus Lactobacillus and 2 within the genus Bacteroides were identified to be associated with a decrease in MRE fecal levels, while higher abundances of an OTU from the genera Bacteroides, Klebsiella and Alistipes were found to be associated with an increase in MRE fecal levels.

8. LV model confirmed beta-lactam treatment, when the identified MRE strains are susceptible to the beta-lactams administered, transplant and chemotherapy as clinical factors associated with MRE intestinal levels.

9. Analysis of caecotroph samples collected from rabbits that develop Epizootic Rabbit Enteropathy (ERE) have confirmed the presence of microbiota dysbiosis in rabbits after the disease onset. Microbiota dysbiosis of ERE rabbits is characterized by the increase in abundance of the genera Clostridium, Turicibacter, Robinsoniella, Coprobacillus, Roseburia, Bacteroides, Alistipes, Akkermansia, Campylobacter and Anaerovorax, and decrease in abundance of the genera Fusibacter and Adlercreutzia.

10. The microbiota dysbiosis in ERE rabbits is not promoted by the reduction of food consumption. Indeed, only 1 change in the microbiota composition in ERE rabbits can be attributed to the decrease in the food consumption (i.e. higher abundance of the genus Anaerovorax). 
11. The microbiota of weaned rabbits evolved over time until reaching a microbiota structure similar to the one observed in adult rabbits on the day 11 post-weaning. No differences could be detected in microbiota structure after weaning in rabbits that develop ERE and those that remain healthy.

12. A unique microbiota change was detected in rabbits that developed ERE, before ERE initiation. Specifically, the day of ERE onset, rabbits that developed ERE had higher levels of a specific OTU (OTU31) belonging to the genus Clostridium. This OTU represents a novel species within the genus Clostridium, which we named Clostridium cuniculi, and it is phylogenetically related with the pathogens $C$. perfringens and $C$. botulinum. The identified $C$. cuniculi encodes virulence factors exoU and hemolysin and the hemagglutinin components of the botulinum toxin operon. 



\section{Abbreviations}

AGC

ANI

AST

AVN

$\mathrm{BSH}$

CD

CDI

$\mathrm{CFU}$

CLSI

CNS

CR

CRE

dNTP

EGS

ERE

ESBL

FDR

FISABIO

FMT

GF

GIT

GM

IBD

$\operatorname{Ig}(\mathrm{A}, \mathrm{E}, \mathrm{G}, \mathrm{D}, \mathrm{M})$

ILC

$\operatorname{IL}(10,17$ or 22$)$

LB
Abundance-based greedy clustering

Average nucleotide identity

Antimicrobial susceptibility testing

Combined antibiotic treatment composed of ampicillin, vancomycin and neomycin

Bile salt hydrolase

Crohn's disease

Clostridium difficile infection

Colony forming unit

Clinical and Laboratory Standard Institute

Central nervous system

Colonization resistance

Carbapenem-resistant Enterobacteriaceae

Deoxynucleoside triphosphates

Equine grass sickness

Epizootic Rabbit Enteropathy

Extended spectrum beta-lactamase

False discovery rate

The Foundation for the Promotion of Health and Biomedical Research of Valencia Region

Fecal microbiota transplant

Germ-free (mice)

Gastrointestinal tract

Fecal anaerobic culture obtained by cultivation of feces in Gut modified cultivation media

Inflammatory bowel disease

Immunoglobulin (A, E, G, D, M)

Innate lymphoid cell

Interleukin $(10,17$ or 22$)$

Luria-Bertani cultivation medium 
LDA Linear Discriminant Analysis

LV Lotka-Volterra mathematical model

MIX5 Mix of 5 bacterial commensal strains isolated from mice feces (Barnesiella, Bacteroides, Mucispirillum, Alistipes, Lactobacillus)

MRE

Multidrug resistant Enterobacteriaceae

MRKP

Multidrug resistant Klebsiella pneumoniae

MRSA

Methicillin-resistant Staphylococcus aureus

OD

Optic density

ORF

Open-reading frame

OTU

Operational taxonomic unit

PBS

Phosphate buffer saline

PCoA

Principal Coordinate of Analysis

PCR

Polymerase-chain reaction

qPCR

Quantitative polymerase-chain reaction

SCFA

Short-chain fatty acid

$\mathrm{SCH}$

Schaedler cultivation media

SINA

Silva Incremental Aligner

SPF

Specific pathogen-free

SP1

Salmonella typhimurium pathogenicity island 1

Th $(1,2$ or 17$)$

T helper cell $(1,2$ or 17$)$

$\mathrm{T}_{\text {reg }}$

Regulatory subset of $\mathrm{T}$ cells

$\mathrm{UC}$

Ulcerative colitis

UPGMA

Unweighted Pair Group Method with Arithmetic mean

VRE

Vancomycin-resistant Enterococcus faecium 




\section{References}

1. Ley RE, Lozupone CA, Hamady M, Knight R, Gordon JI. Worlds within worlds: evolution of the vertebrate gut microbiota. Nat Rev Microbiol. 2008; 6(10): 77688.

2. Gordon JI. Honor Thy Gut Symbionts Redux. Science. 2012; 336: 1251-3.

3. Van den Abbeele P, Van de Wiele T, Verstraete W, Possemiers S. The host selects mucosal and luminal associations of coevolved gut microorganisms: a novel concept. FEMS Microbiol Rev. 2011; 35(4): 681-704.

4. von Martels JZH, Sadaghian Sadabad M, Bourgonje AR, Blokzijl T, Dijkstra G, Faber KN, et al. The role of gut microbiota in health and disease: In vitro modeling of host-microbe interactions at the aerobe-anaerobe interphase of the human gut. Anaerobe. 2017; 44: 3-12.

5. Turnbaugh PJ, Ley RE, Hamady M, Fraser-liggett C, Knight R, Gordon JI. The human microbiome project: exploring the microbial part of ourselves in a changing world. Nature. 2007; 449(7164): 804-10.

6. Sekirov I, Russell S, Antunes L, Finlay BB. Gut microbiota in health and disease. Physiol Rev. 2010; 90(3): 859-904.

7. Schloss PD, Handelsman J. Status of the microbial census. Microbiol Mol Biol Rev. 2004; 68(4): 686-91.

8. Eckburg PB, Bik EM, Bernstein CN, Purdom E, Dethlefsen L, Sargent M, et al. Diversity of the Human Intestinal Microbial Flora. Science. 2005; 308(5728): 1635-8.

9. Lozupone C, Stomabaugh J, Gordon J, Jansson J, Knight R. Diversity , stability and resilience of the human gut microbiota. Nature. 2012; 489(7415): 220-30.

10. Aagaard K, Ma J, Antony KM, Ganu R, Petrosino J, Versalovic J. The Placenta Harbors a Unique Microbiome. Sci Transl Med. 2014; 6(237): 23-30.

11. Collado MC, Rautava S, Aakko J, Isolauri E, Salminen S. Human gut colonisation may be initiated in utero by distinct microbial communities in the placenta and amniotic fluid. Sci Rep. 2016; 6: 23129.

12. Gosalbes MJ, Llop S, Valles Y, Moya A, Ballester F, Francino MP. Meconium microbiota types dominated by lactic acid or enteric bacteria are differentially associated with maternal eczema and respiratory problems in infants. Clin Exp Allergy. 2013; 43(2): 198-211. 
13. Bergström A, Skov TH, Bahl MI, Roager HM, Christensen LB, Ejlerskov KT, et al. Establishment of intestinal microbiota during early life: A longitudinal, explorative study of a large cohort of Danish infants. Appl Environ Microbiol. 2014; 80(9): 2889-900.

14. Voreades N, Kozil A, Weir TL. Diet and the development of the human intestinal microbiome. Front Microbiol. 2014; 5: 494.

15. Marques TM, Wall R, Ross RP, Fitzgerald GF, Ryan CA, Stanton C. Programming infant gut microbiota: Influence of dietary and environmental factors. Curr Opin Biotechnol. 2010; 21(2): 149-56.

16. Rutayisire E, Huang K, Liu Y, Tao F. The mode of delivery affects the diversity and colonization pattern of the gut microbiota during the first year of infants' life: a systematic review. BMC Gastroenterol. 2016; 16(1): 86.

17. Nuriel-Ohayon M, Neuman H, Koren O. Microbial changes during pregnancy, birth, and infancy. Front Microbiol. 2016; 7: 1031.

18. Fallani M, Amarri S, Uusijarvi A, Adam R, Khanna S, Aguilera M, et al. Determinants of the human infant intestinal microbiota after the introduction of first complementary foods in infant samples from five European centres. Microbiology. 2011; 157(5): 1385-92.

19. Odamaki T, Kato K, Sugahara H, Hashikura N, Takahashi S, Xiao J, et al. Agerelated changes in gut microbiota composition from newborn to centenarian: a cross-sectional study. BMC Microbiol. 2016; 16:90.

20. Gerritsen J, Smidt H, Rijkers GT, De Vos WM. Intestinal microbiota in human health and disease: The impact of probiotics. Genes Nutr. 2011; 6(3): 209-40.

21. Swidsinski A, Loening-Baucke V, Lochs H, Hale LP. Spatial organization of bacterial flora in normal and inflamed intestine: a fluorescence in situ hybridization study in mice. World J Gastroenterol. 2005; 11(8): 1131-40.

22. Frank DN, St Amand AL, Feldman RA, Boedeker EC, Harpaz N, Pace NR. Molecular-phylogenetic characterization of microbial community imbalances in human inflammatory bowel diseases. Proc Natl Acad Sci USA. 2007; 104(34): 13780-5.

23. Ubeda C, Bucci V, Caballero S, Djukovic A, Toussaint NC, Equinda M, et al. Intestinal microbiota containing Barnesiella species cures vancomycin-resistant Enterococcus faecium colonization. Infect Immun. 2013 Mar; 81(3): 965-73.

24. Buffie CG, Jarchum I, Equinda M, Lipuma L, Gobourne A, Viale A, et al. Profound alterations of intestinal microbiota following a single dose of 
clindamycin results in sustained susceptibility to Clostridium difficile-induced colitis. Infect Immun. 2012 Jan; 80(1): 62-73.

25. Ley R, Hamady M, Lozupone C, Turnbaugh PJ, Roy RR, Stephen B, et al. Evolution of mammals and their gut microbes. Science. 2008; 320(5883): 164751.

26. Rawls JF, Mahowald MA, Ley RE, Gordon JI. Reciprocal Gut Microbiota Transplants from Zebrafish and Mice to Germ-free Recipients Reveal Host Habitat Selection. Cell. 2006; 127(2): 423-33.

27. Buffie CG, Bucci V, Stein RR, McKenney PT, Ling L, Gobourne A, et al. Precision microbiome reconstitution restores bile acid mediated resistance to Clostridium difficile. Nature. 2014 Oct 22; 0.

28. Zhang Y-J, Li S, Gan R-Y, Zhou T, Xu D-P, Li H-B. Impacts of Gut Bacteria on Human Health and Diseases. Int J Mol Sci. 2015; 16(4): 7493-519.

29. Wu GD, Chen J, Hoffmann C, Bittinger K, Chen Y, Sue A, et al. Linking LongTerm Dietary Patterns with Gut Microbial Enterotypes. Science. 2011; 334(6052): 105-8.

30. David LA, Maurice CF, Carmody RN, Gootenberg DB, Button JE, Wolfe BE, et al. Diet rapidly and reproducibly alters the human gut microbiome. Nature. 2014; 505(7484): 559-63.

31. Lee HC, Jenner AM, Low CS, Lee YK. Effect of tea phenolics and their aromatic fecal bacterial metabolites on intestinal microbiota. Res Microbiol. 2006; 157(9): 876-84.

32. Kato I, Boleij A, Kortman GA, Roelofs R, Djuric Z, Severson RK, et al. Partial associations of dietary iron, smoking and intestinal bacteria with colorectal cancer risk. Nutr Cancer. 2013; 65(2): 169-77.

33. Kohl KD, Amaya J, Passement CA, Dearing MD, Mccue MD. Unique and shared responses of the gut microbiota to prolonged fasting: A comparative study across five classes of vertebrate hosts. FEMS Microbiol Ecol. 2014; 90(3): 883-94.

34. Ramakrishna BS. Role of the gut microbiota in human nutrition and metabolism. J Gastroenterol Hepatol. 2013; 28: 9-17.

35. Lin L, Zhang J. Role of intestinal microbiota and metabolites on gut homeostasis and human diseases. BMC Immunol. 2017; 18:2.

36. Nicholson JK, Holmes E, Kinross J, Burcelin R, Gibson G, Jia W, et al. Host-gut microbiota metabolic interactions. Science. 2012; 336: 1262-7. 
37. D’Aimmo MR, Modesto M, Mattarelli P, Biavati B, Andlid T. Biosynthesis and cellular content of folate in bifidobacteria across host species with different diets. Anaerobe. 2014; 30: 169-77.

38. Ji B, Nielsen J. New insight into the gut microbiome through metagenomics. Adv Genomics Genet. 2015; 5: 77-91.

39. Wang HX, Wang YP. Gut microbiota-brain axis. Chin Med J (Engl). 2016; 129(19): 2373-80.

40. Diaz Heijtz R, Wang S, Anuar F, Qian Y, Björkholm B, Samuelsson A, et al. Normal gut microbiota modulates brain development and behavior. Proc Natl Acad Sci USA. 2011 Feb 15; 108(7): 3047-52.

41. Forsythe P, Sudo N, Dinan T, Taylor VH, Bienenstock J. Mood and gut feelings. Vol. 24, Brain, Behavior and Immunity. Elsevier Inc.; 2010; 9-16.

42. Frost G, Sleeth ML, Sahuri-Arisoylu M, Lizarbe B, Cerdan S, Brody L, et al. The short-chain fatty acid acetate reduces appetite via a central homeostatic mechanism. Nat Commun. 2014 Jan; 5: 3611.

43. Fung TC, Olson CA, Hsiao EY. Interactions between the microbiota, immune and nervous systems in health and disease. Nat Neurosci. 2017; 20(2).

44. Heinz B, E. HR, SM L, H P. The response of the lymphatic tissue to the microbial flora. Studies on germfree mice. Am J Pathol. 1963; 42(4): 471-83.

45. Ganal SC, Sanos SL, Kallfass C, Oberle K, Johner C, Kirschning C, et al. Priming of Natural Killer Cells by Nonmucosal Mononuclear Phagocytes Requires Instructive Signals from Commensal Microbiota. Immunity. 2012; 37: 171-86.

46. Sanos SL, Bui VL, Mortha A, Oberle K, Heners C, Johner C, et al. RORyt and commensal microflora are required for the differentiation of mucosal interleukin 22-producing NKp46+ cells. Nat Immunol. 2009; 10(1): 83-91.

47. Umesaki Y, Setoyama H, Matsumoto S, Okada Y. Expansion of axf T-cell receptor-bearing intestinal intraepithelial lymphocytes after microbial colonization in germ-free mice and its independence from thymus. Immunology. 1993; 79: 327.

48. Lee YK, Mazmanian SK. Has the microbiota played a critical role in the evolution of the adaptive immune system? Science. 2010; 330(6012): 1768-73.

49. Pamer EG. Resurrecting the intestinal microbiota to combat antibiotic-resistant pathogens. Science. 2016; 352(6285): 535-8. 
50. Ubeda C, Djukovic A, Isaac S. Roles of the intestinal microbiota in pathogen protection. Clin Transl Immunol. 2017; 6: 1-10.

51. Hasegawa M, Kamada N, Jiao Y, Liu MZ, Núñez G, Inohara N. Protective role of Commensals against Clostridium difficile Infection via an IL-1ß-Mediated Positive Feedback Loop. J Immunol. 2012; 189(6): 3085-91.

52. Zelante T, Iannitti RG, Cunha C, DeLuca A, Giovannini G, Pieraccini G, et al. Tryptophan catabolites from microbiota engage aryl hydrocarbon receptor and balance mucosal reactivity via interleukin-22. Immunity. 2013; 39(2): 372-85.

53. Ivanov II, Atarashi K, Manel N, Brodie EL, Shima T, Karaoz U, et al. Induction of intestinal Th17 cells by segmented filamentous bacteria. Cell. 2009; 139(3): 48598.

54. Kim M, Qie Y, Park J, Kim CH. Gut Microbial Metabolites Fuel Host Antibody Responses. Cell Host Microbe. 2016; 20(2): 1-13.

55. Rebuffat S. Microcins in action: amazing defence strategies of Enterobacteria. Biochem Soc Trans. 2012; 40(6): 1456-62.

56. Sassone-Corsi M, Nuccio S-P, Liu H, Hernandez D, Vu CT, Takahashi AA, et al. Microcins mediate competition among Enterobacteriaceae in the inflamed gut. Nature. 2016; 0(0).

57. Sassone-Corsi M, Raffatellu M. No Vacancy: How beneficial microbes cooperate with immunity to provide colonization resistance to pathogens. 2015; 194(9): 4081-7.

58. Maltby R, Leatham-Jensen MP, Gibson T, Cohen PS, Conway T. Nutritional Basis for Colonization Resistance by Human Commensal Escherichia coli Strains HS and Nissle 1917 against E. coli O157:H7 in the Mouse Intestine. PLoS One. 2013; 8(1): e53957.

59. Petersen C, Round JL. Defining dysbiosis and its influence on host immunity and disease. Cell Microbiol. 2014; 16(7): 1024-33.

60. Desai MS, Seekatz AM, Koropatkin NM, Kamada N, Hickey CA, Wolter M, et al. A Dietary Fiber-Deprived Gut Microbiota Degrades the Colonic Mucus Barrier and Enhances Pathogen Susceptibility. Cell. 2016; 167(5): 1339-53.

61. Agus A, Denizot J, Thévenot J, Martinez-Medina M, Massier S, Sauvanet P, et al. Western diet induces a shift in microbiota composition enhancing susceptibility to Adherent-Invasive E. coli infection and intestinal inflammation. Sci Rep. 2016; 6: 19032. 
62. Lupp C, Robertson ML, Wickham ME, Sekirov I, Champion OL, Gaynor EC, et al. Host-Mediated Inflammation Disrupts the Intestinal Microbiota and Promotes the Overgrowth of Enterobacteriaceae. Cell Host Microbe. 2007; 2(2): 119-29.

63. Stecher B, Robbiani R, Walker AW, Westendorf AM, Barthel M, Kremer M, et al. Salmonella enterica serovar typhimurium exploits inflammation to compete with the intestinal microbiota. PLoS Biol. 2007; 5(10): 2177-89.

64. Panda S, El Khader I, Casellas F, López Vivancos J, García Cors M, Santiago A, et al. Short-term effect of antibiotics on human gut microbiota. PLoS One. 2014; 9(4).

65. Ubeda C, Taur Y, Jenq RR, Equinda MJ, Son T, Samstein M, et al. Vancomycinresistant Enterococcus domination of intestinal microbiota is enabled by antibiotic treatment in mice and precedes bloodstream invasion in humans. J Clin Invest. 2010; 120(12).

66. Isaac S, Scher JU, Djukovic A, Jimenez N, Littman DR, Abramson SB, et al. Short- and long-term effects of oral vancomycin on the human intestinal microbiota. J Antimicrob Chemother. 2016; 1-9.

67. Rashid M-U, Zaura E, Buijs MJ, Keijser BJF, Crielaard W, Nord CE, et al. Determining the Long-term Effect of Antibiotic Administration on the Human Normal Intestinal Microbiota Using Culture and Pyrosequencing Methods. Clin Infect Dis. 2015 May 15; 60(Suppl 2): S77-84.

68. Pérez-Cobas AE, Gosalbes MJ, Friedrichs A, Knecht H, Artacho A, Eismann K, et al. Gut microbiota disturbance during antibiotic therapy: a multi-omic approach. Gut. 2013 Nov 1; 62(11): 1591-601.

69. Machiels K, Joossens M, Sabino J, De Preter V, Arijs I, Eeckhaut V, et al. A decrease of the butyrate-producing species Roseburia hominis and Faecalibacterium prausnitzii defines dysbiosis in patients with ulcerative colitis. Gut. 2013; 0: 1-9.

70. Amar J, Chabo C, Waget A, Klopp P, Vachoux C, Bermúdez-Humarán LG, et al. Intestinal mucosal adherence and translocation of commensal bacteria at the early onset of type 2 diabetes: Molecular mechanisms and probiotic treatment. EMBO Mol Med. 2011; 3(9): 559-72.

71. Russell SL, Gold MJ, Hartmann M, Willing BP, Thorson L, Wlodarska M, et al. Early life antibiotic-driven changes in microbiota enhance susceptibility to allergic asthma. EMBO Rep. 2012; 13(5): 440-7. 
72. Lynch S V., Pedersen O. The Human Intestinal Microbiome in Health and Disease. N Engl J Med. 2016; 375(24): 2369-79.

73. Joossens M, Huys G, Cnockaert M, De Preter V, Verbeke K, Rutgeerts P, et al. Dysbiosis of the faecal microbiota in patients with Crohn's disease and their unaffected relatives. Gut. 2011; 60(5): 631-7.

74. Weiss GA, Hennet T. Mechanisms and consequences of intestinal dysbiosis. Cell Mol Life Sci. 2017; 0(0):0.

75. Lyte M, Li W, Opitz N, Gaykema RPA, Goehler LE. Induction of anxiety-like behavior in mice during the initial stages of infection with the agent of murine colonic hyperplasia Citrobacter rodentium. Physiol Behav. 2006; 89(3): 350-7.

76. Liss BJ, Vehreschild JJ, Cornely OA, Hallek M, Fätkenheuer G, Wisplinghoff H, et al. Intestinal colonisation and blood stream infections due to vancomycinresistant enterococci (VRE) and extendedspectrum beta-lactamase- producing Enterobacteriaceae (ESBLE) in patients with haematological and oncological malignancies. Infection. 2012; 40(6): 613-9.

77. Bibbò S, Lopetuso LR, Ianiro G, Di Rienzo T, Gasbarrini A, Cammarota G. Role of microbiota and innate immunity in recurrent Clostridium difficile infection. $\mathrm{J}$ Immunol Res. 2014 Jan; 2014: 462740.

78. Mittal G, Gaind R, Kumar D, Kaushik G, Gupta KB, Verma PK, et al. Risk factors for fecal carriage of carbapenemase producing Enterobacteriaceae among intensive care unit patients from a tertiary care center in India. BMC Microbiol. 2016; 16(1): 138.

79. Thiemann S, Smith N, Strowig T. Antibiotics and the Intestinal Microbiome: Individual Responses, Resilience of the Ecosystem, and the Susceptibility to Infections. Life Sci J. 2009; 6(4): 23-7.

80. Magiorakos AP, Srinivasan A, Carey RB, Carmeli Y, Falagas ME, Giske CG, et al. Multidrug-resistant, extensively drug-resistant and pandrug-resistant bacteria: An international expert proposal for interim standard definitions for acquired resistance. Clin Microbiol Infect. 2012; 18(3): 268-81.

81. Karaiskos I, Giamarellou H. Multidrug-resistant and extensively drug-resistant Gram-negative pathogens : current and emerging therapeutic approaches. Expert Opin Pharmacother. 2014; 15(10): 1351-70.

82. European Centre for Disease Prevention and Control. Antimicrobial resistance surveillance in Europe. 2017. 
83. Woerther PL, Micol JB, Angebault C, Pasquier F, Pilorge S, Bourhis JH, et al. Monitoring antibiotic-resistant enterobacteria faecal levels is helpful in predicting antibiotic susceptibility of bacteraemia isolates in patients with haematological malignancies. J Med Microbiol. 2015; 64(7): 676-81.

84. Donskey CJ. The Role of the Intestinal Tract As a Source for Transmission of Nosocomial Pathogens. Clin Infect Dis. 2004; 39: 219-26.

85. Caballero S, Carter R, Ke X, Sušac B, Leiner IM, Kim GJ, et al. Distinct but Spatially Overlapping Intestinal Niches for Vancomycin-Resistant Enterococcus faecium and Carbapenem-Resistant Klebsiella pneumoniae. PLoS Pathog. 2015 Sep; 11(9): 1005132.

86. Torres-Gonzalez P, Cervera-Hernandez ME, Niembro-Ortega MD, Leal-Vega F, Cruz-Hervert LP, García-García L, et al. Factors associated to prevalence and incidence of carbapenem-resistant enterobacteriaceae fecal carriage: A cohort study in a Mexican tertiary care hospital. PLoS One. 2015; 10(10): 1-13.

87. Hoyen CK, Pultz NJ, Paterson DL, Aron C, Donskey CJ, Aron DC. Effect of Parenteral Antibiotic Administration on Establishment of Intestinal Colonization in Mice by Klebsiella pneumoniae Strains Producing Extended-Spectrum $\beta$ Lactamases. Antimicrob Agents Chemother. 2003; 47(11): 3610-2.

88. Barman M, Unold D, Shifley K, Amir E, Hung K, Bos N, et al. Enteric salmonellosis disrupts the microbial ecology of the murine gastrointestinal tract. Infect Immun. 2008; 76(3): 907-15.

89. Bauerl C, Collado MC, Zuniga M, Blas E, Martinez GP. Changes in cecal microbiota and mucosal gene expression revealed new aspects of epizootic rabbit enteropathy. PLoS One. 2014; 9(8).

90. Haligur M, Ozmen O, Demir N. Pathological and Ultrastructural Studies on Mucoid Enteropathy in New Zealand Rabbits. J Exot Pet Med. 2009 Jul; 18(3): 224-8.

91. Rodríguez-De Lara R, Cedillo-Peláez C, Constantino-Casas F, Fallas-López M, Cobos-Peralta M, Gutiérrez-Olvera C, et al. Studies on the evolution, pathology, and immunity of commercial fattening rabbits affected with epizootic outbreaks of diarrhoeas in Mexico: a case report. Res Vet Sci. 2008 Apr; 84: 257-68.

92. Licois D, Wyers M, Coudert P. Epizootic Rabbit Enteropathy: experimental transmission and clinical characterization. Vet Res. 2005; 36: 601-13.

93. Szalo IM, Lassence C, Licois D, Coudert P, Poulipoulis A, Vindevogel H, et al. Fractionation of the reference inoculum of epizootic rabbit enteropathy in 
discontinuous sucrose gradient identifies aetiological agents in high density fractions. Vet J. 2007 May; 173(3): 652-7.

94. Marlier D, Dewrée R, Lassence C, Licois D, Mainil J, Coudert P, et al. Infectious agents associated with epizootic rabbit enteropathy: isolation and attempts to reproduce the syndrome. Vet J. 2006 Nov; 172(3): 493-500.

95. Romero C, Nicodemus N, Deblas JC. Dietary level of fibre and age at weaning affect the proliferation of Clostridium perfringens in the caecum, the incidence of Epizootic Rabbit Enteropathy and the performance of fattening rabbits. Anim Feed Sci Technol. 2009; 153(1-2): 131-40.

96. Buffie CG, Pamer EG. Microbiota-mediated colonization resistance against intestinal pathogens. Nat Rev Immunol. 2013; 13(11): 790-801.

97. Landy J, Al-Hassi HO, McLaughlin SD, Walker AW, Ciclitira PJ, Nicholls RJ, et al. Review article: Faecal transplantation therapy for gastrointestinal disease. Aliment Pharmacol Ther. 2011; 34(4): 409-15.

98. van Nood E, Vrieze A, Nieuwdorp M, Fuentes S, Zoetendal EG, de Vos WM, et al. Duodenal infusion of donor feces for recurrent Clostridium difficile. N Engl J Med. 2013; 368(5): 407-15.

99. Petrof EO, Gloor GB, Vanner SJ, Weese SJ, Carter D, Daigneault MC, et al. Stool substitute transplant therapy for the eradication of Clostridium difficile infection: “RePOOPulating” the gut. Microbiome. 2013; 1(1): 3.

100. Lawley TD, Clare S, Walker AW, Stares MD, Connor TR, Raisen C, et al. Targeted restoration of the intestinal microbiota with a simple, defined bacteriotherapy resolves relapsing Clostridium difficile disease in mice. PLoS Pathog. 2012 Jan; 8(10): e1002995.

101. Michelland R, Combes S, Cauquil L, Gidenne T, Monteils V. Characterization of bacterial communities in caeum, hard and soft feces of rabbit using 16S rRNA genes capillary electrophoresis single-strand conformation polymorphism (cesscp). 9th World Rabbit Congr. 2008; 1025-30.

102. Thompson JA, Oliveira RA, Djukovic A, Ubeda C, Xavier KB. Manipulation of the Quorum Sensing Signal AI-2 Affects the Antibiotic-Treated Gut Microbiota. Cell Rep. 2015; 10: 1-11.

103. Schloss PD, Westcott SL, Ryabin T, Hall JR, Hartmann M, Hollister EB, et al. Introducing mothur: open-source, platform-independent, community-supported software for describing and comparing microbial communities. Appl Environ Microbiol. 2009 Dec; 75(23): 7537-41. 
104. Pruesse E, Quast C, Knittel K, Fuchs BM, Ludwig W, Peplies J, et al. SILVA: a comprehensive online resource for quality checked and aligned ribosomal RNA sequence data compatible with ARB. Nucleic Acids Res. 2007 Jan; 35(21): 718896.

105. Haas BJ, Gevers D, Earl AM, Feldgarden M, Ward D V, Giannoukos G, et al. Chimeric 16S rRNA sequence formation and detection in Sanger and 454pyrosequenced PCR amplicons. Genome Res. 2011 Mar; 21(3): 494-504.

106. Dethlefsen L, Huse S, Sogin ML, Relman DA. The Pervasive Effects of an Antibiotic on the Human Gut Microbiota, as Revealed by Deep 16S rRNA Sequencing. PLoS Genet. 2008; 4(11).

107. Wang Q, Garrity GM, Tiedje JM, Cole JR. Naive Bayesian classifier for rapid assignment of rRNA sequences into the new bacterial taxonomy. Appl Environ Microbiol. 2007 Aug; 73(16): 5261-7.

108. Edgar RC, Haas BJ, Clemente JC, Quince C, Knight R. UCHIME improves sensitivity and speed of chimera detection. 2011; 27(16): 2194-200.

109. Westcott SL, Schloss PD. De novo clustering methods outperform reference-based methods for assigning 16S rRNA gene sequences to operational taxonomic units. Peer J. 2015; 3: e1487.

110. Martin M. Cutadapt removes adapter sequences from high-throughput sequencing reads. $2011 ; 17(1)$ : 17-9.

111. Modolo L, Lerat E. UrQt: an efficient software for the Unsupervised Quality trimming of NGS data. BMC Bioinformatics. 2015; 16(1): 137.

112. Bankevich A, Nurk S, Antipov D, Gurevich AA, Dvorkin M, Kulikov AS, et al. SPAdes: A New Genome Assembly Algorithm and Its Applications to Single-Cell Sequencing. J Comput Biol. 2012; 19(5): 455-77.

113. Seemann T. Prokka: Rapid prokaryotic genome annotation. Bioinformatics. 2014; 30(14): 2068-9.

114. Finn RD, Bateman A, Clements J, Coggill P, Eberhardt RY, Eddy SR, et al. Pfam: The protein families database. Nucleic Acids Res. 2014; 42(D1): 222-30.

115. Huerta-Cepas J, Szklarczyk D, Forslund K, Cook H, Heller D, Walter MC, et al. EGGNOG 4.5: A hierarchical orthology framework with improved functional annotations for eukaryotic, prokaryotic and viral sequences. Nucleic Acids Res. 2016; 44(D1): D286-93. 
116. Ogata H, Goto S, Sato K, Fujibuchi W, Bono H, Kanehisa M. KEGG: Kyoto encyclopedia of genes and genomes. Nucleic Acids Res. 1999; 27(1): 29-34.

117. Finn RD, Clements J, Eddy SR. HMMER web server: Interactive sequence similarity searching. Nucleic Acids Res. 2011; 39(W): 29-37.

118. Katoh K, Misawa K, Kuma K, Miyata T. MAFFT: a novel method for rapid multiple sequence alignment based on fast Fourier transform. Nucleic Acids Res. 2002; 30(14): 3059-66.

119. Richter M, Rosselló-Móra R. Shifting the genomic gold standard for the prokaryotic species definition. Proc Natl Acad Sci USA. 2009; 106(45): 1912631.

120. Ondov BD, Treangen TJ, Melsted P, Mallonee AB, Bergman NH, Koren S, et al. Mash: fast genome and metagenome distance estimation using MinHash. Genome Biol. 2016; 17(1): 132.

121. Touchon M, Cury J, Yoon EJ, Krizova L, Cerqueira GC, Murphy C, et al. The genomic diversification of the whole Acinetobacter genus: Origins, mechanisms, and consequences. Genome Biol Evol. 2014; 6(10): 2866-82.

122. Nguyen LT, Schmidt HA, Von Haeseler A, Minh BQ. IQ-TREE: A fast and effective stochastic algorithm for estimating maximum-likelihood phylogenies. Mol Biol Evol. 2015; 32(1): 268-74.

123. Chakraborty A, Ghosh S, Chowdhary G, Maulik U, Chakrabarti S. DBETH: A database of bacterial exotoxins for human. Nucleic Acids Res. 2012; 40(D1): 61520 .

124. Segata N, Izard J, Waldron L, Gevers D, Miropolsky L, Garrett WS, et al. Metagenomic biomarker discovery and explanation. Genome Biol. 2011; 12(6): R60.

125. Benjamini Y; Hochberg Y. Controlling the False Discovery Rate: A Practical and Powerful approach to Multiple Testing. J R Stat Soc. 1995; 57: 289-300.

126. Friedman J, Hastie T, Tibshirani R. Regularization Paths for Generalized Linear Models via Coordinate Descent. J Stat Softw. 2010; 33(1): 1-22.

127. Stein RR, Bucci V, Toussaint NC, Buffie CG, Rätsch G, Pamer EG, et al. Ecological modeling from time-series inference: insight into dynamics and stability of intestinal microbiota. PLoS Comput Biol. 2013 Dec; 9(12): e1003388. 
128. Taur Y, Xavier JB, Lipuma L, Ubeda C, Goldberg J, Gobourne A, et al. Intestinal domination and the risk of bacteremia in patients undergoing allogeneic hematopoietic stem cell transplantation. Clin Infect Dis. 2012 Oct; 55(7): 905-14.

129. Gough E, Shaikh H, Manges AR. Systematic review of intestinal microbiota transplantation (fecal bacteriotherapy) for recurrent Clostridium difficile infection. Clin Infect Dis. 2011; 53(10): 994-1002.

130. Huybens N, Houeix J, Licois D, Mainil J, Marlier D. Pyrosequencing of epizootic rabbit enteropathy inocula and rabbit caecal samples. Vet J. 2013; 196(1): 109-10.

131. Zhang L, Lin WJ, Li S, Aoki KR. Complete DNA sequences of the botulinum neurotoxin complex of Clostridium botulinum type A-Hall (Allergan) strain. Gene. 2003; 315(1-2): 21-32.

132. Colovic MB, Krstic DZ, Lazarevic-Pasti TD, Bondzic AM, Vasic VM. Acetylcholinesterase Inhibitors: Pharmacology and Toxicology. Curr Neuropharmacol. 2013; 11(3): 315-35.

133. van der Waaij D, Berghuis-de Vries JM, Lekkerkerk-van der Wees JEC. Colonization resistance of the digestive tract in conventional and antibiotic-treated mice. J Hyg (Lond). 1971; 69(3): 405-11.

134. Citron DM, Tyrrell KL, Merriam CV, Goldstein EJC. In vitro activities of CB183,315, vancomycin, and metronidazole against 556 strains of Clostridium difficile, 445 other intestinal anaerobes, and 56 Enterobacteriaceae species. Antimicrob Agents Chemother. 2012; 56(3): 1613-5.

135. Credito KL, Appelbaum PC. Activity of OPT-80, a novel macrocycle, compared with those of eight other agents against selected anaerobic species. Antimicrob Agents Chemother. 2004; 48(11): 4430-4.

136. Finegold SM, Molitoris D, Väisänen ML. Study of the in vitro activities of rifaximin and comparator agents against 536 anaerobic intestinal bacteria from the perspective of potential utility in pathology involving bowel flora. Antimicrob Agents Chemother. 2009; 53(1): 281-6.

137. Goldstein EJC, Citron DM, Tyrrell KL, Merriam CV. Comparative In Vitro activities of SMT19969, a new antimicrobial agent, against Clostridium difficile and 350 Gram-Positive and Gram-Negative aerobic and anaerobic intestinal flora isolates. Antimicrob Agents Chemother. 2013; 57(10): 4872-6.

138. Gonzales M, Pepin J, Frost EH, Carrier JC, Sirard S, Fortier L-C, et al. Faecal pharmacokinetics of orally administered vancomycin in patients with suspected Clostridium difficile infection. BMC Infect Dis. 2010; 10(1): 363. 
139. Lewis BB, Buffie CG, Carter R, Leiner I, Toussaint NC, Miller L, et al. Loss of microbiota-mediated colonization resistance to Clostridium difficile infection is greater following oral vancomycin as compared with metronidazole. J Infect Dis. 2015; 1-31.

140. Donskey CJ. Antibiotic regimens and intestinal colonization with antibioticresistant gram-negative bacilli. ClinInfectDis. 2006; 43 Suppl 2(1537-6591 (Electronic)): S62-9.

141. Goodman AL, Kallstrom G, Faith JJ, Reyes A, Moore A, Dantas G, et al. Extensive personal human gut microbiota culture collections characterized and manipulated in gnotobiotic mice. Proc Natl Acad Sci USA. 2011; 108(15): 62527.

142. Sakamoto M, Lan PTN, Benno Y. Barnesiella viscericola gen. nov., sp. nov., a novel member of the family Porphyromonadaceae isolated from chicken caecum. Int J Syst Evol Microbiol. 2007; 57(2): 342-6.

143. Roe AJ, O’Byrne C, McLaggan D, Booth IR. Inhibition of Escherichia coli growth by acetic acid: a problem with methionine biosynthesis and homocysteine toxicity. Microbiology. 2002; 148: 2215-22.

144. Karska-Wysocki B, Bazo M, Smoragiewicz W. Antibacterial activity of Lactobacillus acidophilus and Lactobacillus casei against methicillin-resistant Staphylococcus aureus (MRSA). Microbiol Res. 2010; 165(8): 674-86.

145. Kumar M, Dhaka P, Vijay D, Vergis J, Mohan V, Kumar A, et al. Antimicrobial effects of Lactobacillus plantarum and Lactobacillus acidophilus against multidrug-resistant enteroaggregative Escherichia coli. Int J Antimicrob Agents. 2016; 48(3): 265-70.

146. Castillo NA, Perdigon G, de Moreno de Leblanc A. Oral administration of a probiotic Lactobacillus modulates cytokine production and TLR expression improving the immune response against Salmonella enterica serovar Typhimurium infection in mice. BMC Microbiol. 2011; 11(1): 177.

147. Joyce SA, MacSharry J, Casey PG, Kinsella M, Murphy EF, Shanahan F, et al. Regulation of host weight gain and lipid metabolism by bacterial bile acid modification in the gut. Proc Natl Acad Sci USA. 2014 May 20; 111(20): 7421-6.

148. Song DF, Zhu MY, Gu Q. Purification and characterization of Plantaricin ZJ5, a new bacteriocin produced by Lactobacillus plantarum ZJ5. PLoS One. 2014; 9(8): e105549. 
149. Etienne-Mesmin L, Livrelli V, Privat M, Denis S, Cardot JM, Alric M, et al. Effect of a new probiotic Saccharomyces cerevisiae strain on survival of Escherichia coli O157:H7 in a dynamic gastrointestinal model. Appl Environ Microbiol. 2011; 77(3): 1127-31.

150. Jørgensen KM, Wassermann T, Jensen PØ, Hengzuang W, Molin S, Høiby N, et al. Sublethal ciprofloxacin treatment leads to rapid development of high-level ciprofloxacin resistance during long-term experimental evolution of Pseudomonas aeruginosa. Antimicrob Agents Chemother. 2013; 57(9): 4215-21.

151. Redgrave LS, Sutton SB, Webber MA, Piddock LJ V. Fluoroquinolone resistance: Mechanisms, impact on bacteria, and role in evolutionary success. Trends Microbiol. 2014; 22(8): 438-45.

152. Giacobbe DR, Del Bono V, Trecarichi EM, De Rosa FG, Giannella M, Bassetti M, et al. Risk factors for bloodstream infections due to colistin-resistant KPCproducing Klebsiella pneumoniae: Results from a multicenter case-control-control study. Clin Microbiol Infect. 2015; 21(12): 1106.e1-1106.e8.

153. van Vliet MJ, Harmsen HJM, de Bont ESJM, Tissing WJE. The role of intestinal microbiota in the development and severity of chemotherapy-induced mucositis. PLoS Pathog. 2010; 6(5): 1-7.

154. Zhu X-X, Yang X-J, Chao Y-L, Zheng H-M, Sheng H-F, Liu H-Y, et al. The potential effect of oral microbiota in the prediction of mucositis during radiotherapy for nasopharyngeal carcinoma. EBioMedicine. 2017; 18: 23-31.

155. Kageyama A, Benno Y. Coprobacillus catenaformis Gen. Nov., Sp. Nov., a New Genus and Species Isolated from human feces. Microbiol Immunol. 2000; 44(1): 23-8.

156. Walsh CJ, Guinane CM, O’ Toole PW, Cotter PD. A Profile Hidden Markov Model to investigate the distribution and frequency of LanB-encoding lantibiotic modification genes in the human oral and gut microbiome. PeerJ. 2017; 5: e3254.

157. Saulnier MD, Kevin R, Ann MT, Alejandra DM, Debasmita M, Sabeen R, et al. Gastrointestinal Microbiome Signatures of. Gastroenterology. 2011; 141(5): 178291.

158. Jiang H, Ling Z, Zhang Y, Mao H, Ma Z, Yin Y, et al. Altered fecal microbiota composition in patients with major depressive disorder. Brain Behav Immun. 2015; 48: 186-94. 
159. Huang Y-L, Chassard C, Hausmann M, von Itzstein M, Hennet T. Sialic acid catabolism drives intestinal inflammation and microbial dysbiosis in mice. Nat Commun. 2015 Jan; 6: 8141.

160. Kamada N, Kim Y-G, Sham HP, Vallance BA, Puente JL, Martens EC, et al. Regulated Virulence Controls the Ability of a Pathogen to Compete with the Gut Microbiota. Science. 2012; 336(6086): 1325-9.

161. Li X, Min X, Tsuzuki Y, Sakaguchi E. Effect of indigestible sugars on nitrogen utilization in adult rabbits. Anim Sci J. 2011; 82(2): 296-301.

162. Queipo-Ortuño MI, Seoane LM, Murri M, Pardo M, Gomez-Zumaquero JM, Cardona F, et al. Gut Microbiota Composition in Male Rat Models under Different Nutritional Status and Physical Activity and Its Association with Serum Leptin and Ghrelin Levels. PLoS One. 2013; 8(5).

163. Cottrell DF, McGorum BC, Pearson GT. The neurology and enterology of equine grass sickness: A review of basic mechanisms. Neurogastroenterol Motil. 1999; 11(2): 79-92.

164. Miyashita S-I, Sagane Y, Suzuki T, Matsumoto T, Niwa K, Watanabe T. "NonToxic” Proteins of the Botulinum Toxin Complex Exert In-vivo Toxicity. Sci Rep. 2016; 6: 31043.

165. Dewrée R, Meulemans L, Lassence C, Desmecht D, Ducatelle R, Mast J, et al. Experimentaly induced epizootic rabbit enteropathy: clinical, histopathological, ultrastructural, bacterioulogical and haematological findings. World Rabbit Sci. 2007; 15: 91-102.

166. Coudert P, Licois D. Study of early phenomena during experimental epizootic rabbit enteropathy: preliminary results. Proc 8th World Rabbit Congr. 2004; 5205. 



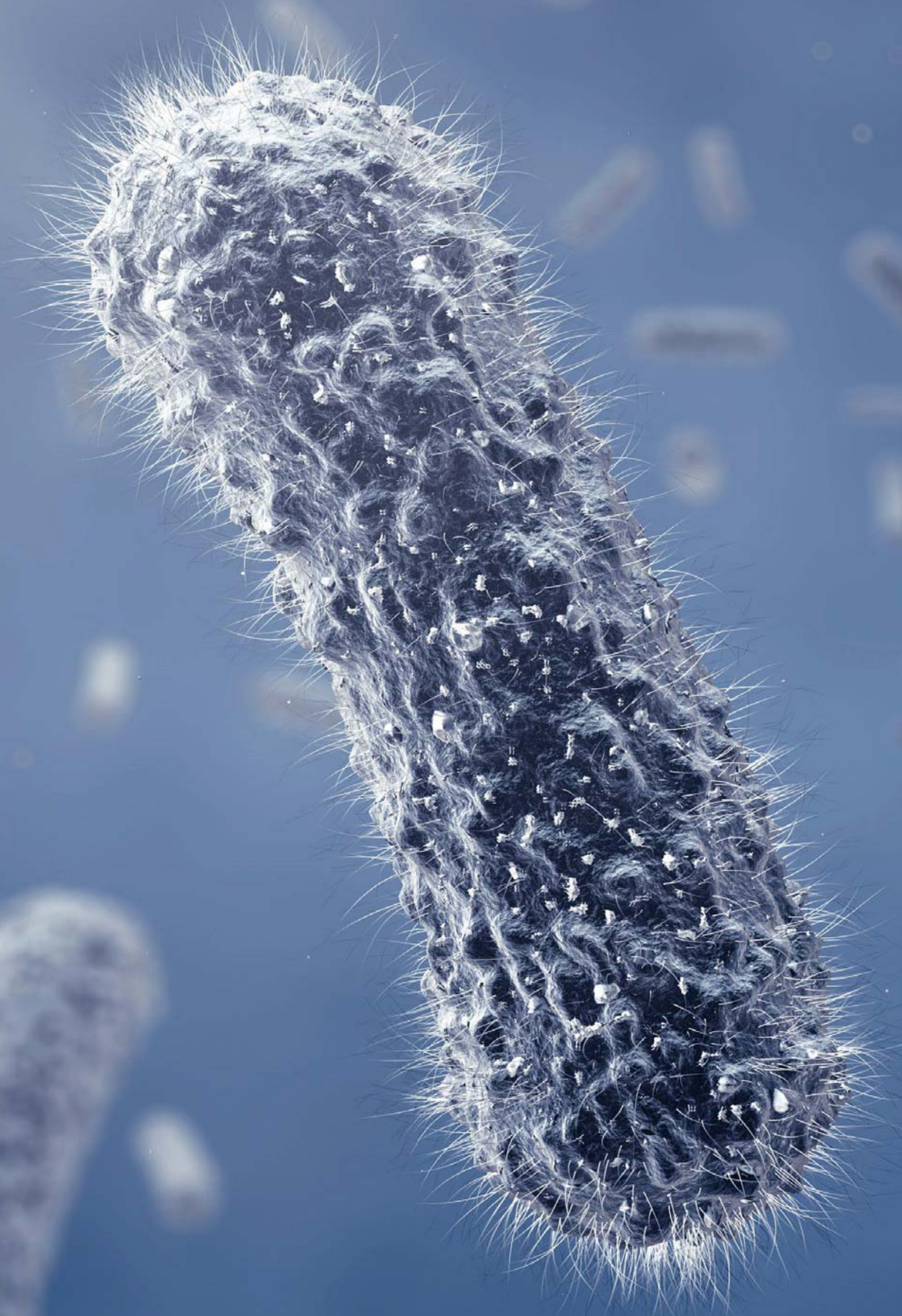

